\title{
Analyzing Options for Airborne Emergency Wireless Communications
}

Juan Deaton

Michael Schmitt

Shane Cherry

Curt Papke

March 2008

Idaho National Laboratory

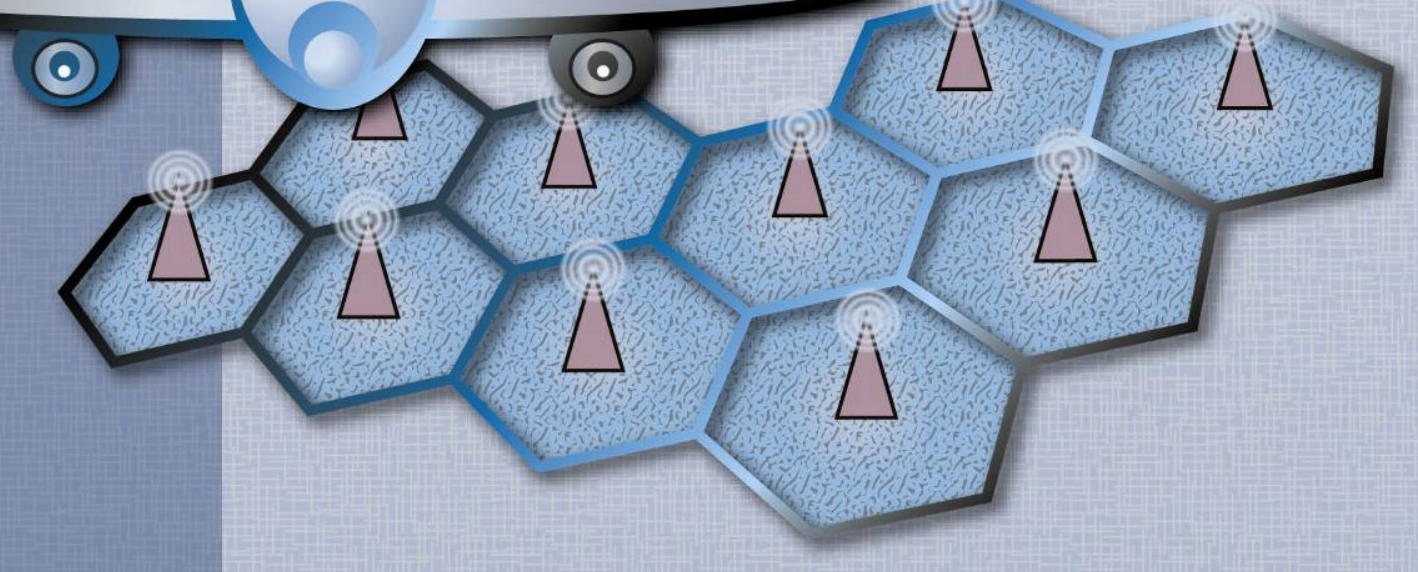




\section{DISCLAIMER}

This information was prepared as an account of work sponsored by an agency of the U.S. Government. Neither the U.S. Government nor any agency thereof, nor any of their employees, makes any warranty, expressed or implied, or assumes any legal liability or responsibility for the accuracy, completeness, or usefulness, of any information, apparatus, product, or process disclosed, or represents that its use would not infringe privately owned rights. References herein to any specific commercial product, process, or service by trade name, trade mark, manufacturer, or otherwise, does not necessarily constitute or imply its endorsement, recommendation, or favoring by the U.S. Government or any agency thereof. The views and opinions of authors expressed herein do not necessarily state or reflect those of the U.S. Government or any agency thereof. 


\title{
Analyzing Options for Airborne Emergency Wireless Communication
}

\author{
Juan Deaton \\ Michael Schmitt \\ Shane Cherry \\ Curt Papke
}

March 2008

\begin{abstract}
Idaho National Laboratory
Idaho Falls, Idaho 83415
\end{abstract}

Prepared for the

U.S. Department of Defense

and for the

U.S. Department of Energy

Under DOE Idaho Operations Office

Contract DE-AC07-05ID1451 



\title{
Analyzing Options for Airborne Emergency Wireless Communication
}

\author{
INL/EXT-08-13871
}

Approved by:

Name

Title [optional]

Name

Title [optional]

Name

Title [optional]

Name

Title [optional]

Date 



\begin{abstract}
In the event of large-scale natural or manmade catastrophic events, access to reliable and enduring commercial communication systems is critical. Hurricane Katrina provided a recent example of the need to ensure communications during a national emergency. To ensure that communication demands are met during these critical times, Idaho National Laboratory (INL) under the guidance of United States Strategic Command has studied infrastructure issues, concerns, and vulnerabilities associated with an airborne wireless communications capability. Such a capability could provide emergency wireless communications until public/commercial nodes can be systematically restored. This report focuses on the airborne cellular restoration concept; analyzes basic infrastructure requirements; identifies related infrastructure issues, concerns, and vulnerabilities and offers recommended solutions.
\end{abstract}




\section{EXECUTIVE SUMMARY}

After Hurricane Katrina, all forms of terrestrial communications networks were severely debilitated. Damage to first responder networks caused multiple problems in command, control, and rescue operations. This unprecedented damage to telecommunications networks has kindled a rejuvenated interest in deployable emergency telecommunication systems within industry and government. An Airborne Communication Node $(\mathrm{ACN})$ for emergency communications has a great potential for assisting in a catastrophic event.

According to the Bipartisan Committee Investigation of Response to Hurricane Katrina, emergency communications needed during hurricane Katrina could be grouped into these three categories: (1) First Responder Communications, (2) Command and Control, (3) Critical Infrastructure Restoration

During a catastrophic incident, all forms of terrestrial communications will be either completely destroyed or severely debilitated. 911 call loading will experience severe peaks, however due to the low call hold time 30 simultaneous channels should be adequate for a worst case scenario. 911 call volumes will be very high which may require an alternative answering technique rather than a PSAP operator. Due to limited battery life and availability of terrestrial assets the ACN will be most effective for a two-week period immediately following the incident. Based on the population data from hurricane Katrina, estimates and calculations, 350 simultaneous channels are needed for a daylight rescue and 207 simultaneous channels are needed for continuous rescue case. A payload of $1000 \mathrm{lbs}$ could support 300 simultaneous channels using COTS technologies.

For an emergency ACN platform to be the most effective, it is important that its capabilities are integrated into a complete emergency communications architecture. Depending on aircraft payloads capabilities, configuration choices for equipment will be different. However, all subsystems of the architecture will still be required. These subsystems are cellular, first responder and backhaul. These subsystems have three main configuration options: 1) the system can be deployed as an aircraft repeater, 2) a complete system on an aircraft or 3) a Base Transceiver Station (BTS) on an aircraft. Using any of these options for the ACN, it is possible to provide in-network and out-of-network calls using an ACN.

The cellular communications available must also be representative of the current cellular ecosystem in order that existing devices can communicate with both the Global System for Mobile (GSM) and Code Division Multiple Access (CDMA) technologies. GSM and CDMA technologies form the two primary cellular technology families with GSM being the worldwide dominant technology. In the U.S., they have almost equal market share.

E911 capabilities should be leveraged to assist in disaster relief efforts. This capability being fielded nation-wide allows information such as caller location to be sent to a Public Safety Answering Point (PSAP) and the Emergency Operations Center (EOC). To alleviate PSAP operator overload, a method for text-based communications for 911 efforts should be developed. This would entail building a new capability for 911 services and new functionality on handsets.

Deployment of first responder communications is challenging due to the interoperability problems. However, there are short-term options which can be utilized such as using a standard phone handout and deploying audio gateways. Solutions to this problem would include utilizing future technologies that will be deployed in the D block of the $700 \mathrm{Mhz}$ reallocation or utilizing a Software Defined Radio system.

Although there are many commercial off-the-shelf (COTS) solutions for antennas, the most effective way to provide ideal coverage and capacity is to use a specialized antenna. Cellular antennas should utilize cross-polarization diversity in order to reduce the antenna footprint while increasing sensitivity. Antennas should be capable of transmitting more than one carrier. Due to the gains in coverage area, antennas should

be adaptive systems. Antenna beam width should be such that the horizontal and vertical beam widths are at 
least $80^{\circ}$. First responder communications may use an Omni antenna. First responder communications antenna should be capable of transmitting and receiving in the $150 \mathrm{MHz}, 450 \mathrm{MHz}, 700 \mathrm{MHz}$, and $800 \mathrm{MHz}$ bands. Permanently-mounted antennas will provide the best continuity for planning flight operations.

In terms of coverage, to reduce the Doppler Effect, speed of aircraft should be kept at the minimumallowable speed for cruising and maintaining a circular flight path. Multiple sector systems will provide greater coverage. Antenna down tilt and the vertical and horizontal beam width will define the coverage area. Coverage area can be maximized by reducing speed and increasing altitude. The sector-carrier capacity will be no more than 80 users or $90 \%$ loading to maintain the coverage area. Additional users may be denied service. Adding more carriers to the same sector will increase the number of users allowed. For modeling purposes, GSM limits should be used to guarantee service.

There are many policy issues which must be resolved before deployment of any type of ACN. Radio frequency spectrum is limited resource and is a very valuable for cellular operators. Agreements must be reached with the respective license holders before cellular spectrum can be used. A phone numbering plan or directory must be developed along with a separate Home Location Register to allow users to roam onto the private network. Federal Aviation Administration (FAA) policies must be set in place to coordinate how an ACN should be deployed.

There are several commercial vendors offering ACN solutions. Aerofone, Platforms Wireless, and TECORE are a few vendors who can provide these turnkey solutions. However, agreements with cellular operators must still be reached for frequency utilization. This could mean soliciting the help of an agent to coordinate issues with frequency use. United States Northern Command (USNORTHCOM), Air National Guard, and Federal Emergency Management Agency (FEMA) all use an agent (Rivada Networks) to coordinate frequency usage with cellular carriers. 


\section{ACKNOWLEDGMENTS}

In creation of this report there were several people who gave their time gladly and freely to help the INL develop this report. The following people were very helpful in the development and added to our conclusions. We would like to thank them for their efforts.

AT\&T wireless: Mr. Brian Daly, Mr. Peter Musgrove and Mr. Ming Zhang.

National Communications Systems: Mr. Bob Lee

Rivada Networks: Mr. Jamie Tackett and Mr. Bob Duncan

National Institute of Information and Communications Technology of Japan: Dr. Hiroyuki Tsuji, Dr. Derek Gray

University of York: Dr. David Grace

Northrop Grumman: Paul Zavidak, Claude Hashem

Qualcomm: Robert Olson

City of New Orleans: Dan Gilbert 


\section{TABLE OF CONTENTS}

\begin{tabular}{|c|c|c|c|}
\hline \multicolumn{4}{|c|}{ IBSTRACT .............. } \\
\hline \multirow{2}{*}{\multicolumn{4}{|c|}{$\begin{array}{l}\text { EXECUTIVE SUMMARY } \\
\text { ACKNOWLEDGMENTS }\end{array}$}} \\
\hline & & & \\
\hline & \multicolumn{3}{|c|}{ Introduction } \\
\hline & 1.1 & \multicolumn{2}{|c|}{ Overview... } \\
\hline & 1.2 & \multicolumn{2}{|r|}{ Objectives...1 } \\
\hline & 1.3 & \multicolumn{2}{|c|}{ Organization } \\
\hline & \multicolumn{3}{|c|}{ Wireless Networks ...... } \\
\hline & 2.1 & \multicolumn{2}{|c|}{ Introduction........ } \\
\hline & 2.2 & \multicolumn{2}{|c|}{ 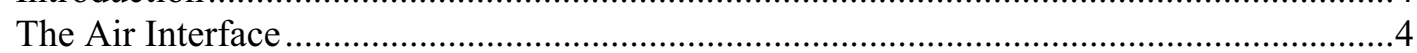 } \\
\hline & 2.3 & \multicolumn{2}{|c|}{ Cellular System Architectures.... } \\
\hline & 2.4 & \multicolumn{2}{|r|}{ Cellular Technology: Generational Systems } \\
\hline & 2.5 & \multicolumn{2}{|r|}{ US Cellular Technology Composition and Operators........................................... } \\
\hline & 2.6 & \multicolumn{2}{|c|}{ Disruptive Technologies: WiFi and WiMAX } \\
\hline & 2.7 & \multicolumn{2}{|c|}{ Technology Trends } \\
\hline & 2.8 & \multicolumn{2}{|c|}{ Summary } \\
\hline & \multicolumn{3}{|c|}{ 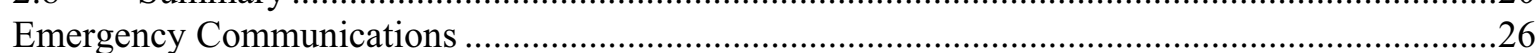 } \\
\hline & \multirow[t]{3}{*}{3.1} & \multicolumn{2}{|c|}{ E911 ........................... } \\
\hline & & 3.1 .1 & ne E911 ... \\
\hline & & 3.1 .2 & .27 \\
\hline & 3.2 & Next & aration 911 \\
\hline & & 3.2 .1 & Network Archi \\
\hline & & 3.2 . & 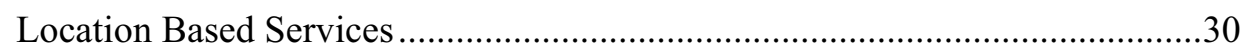 \\
\hline & & 3.2 .3 & NG 9-1-1 Impacts to the ACN \\
\hline & 3.3 & Public & ety Wireless Netw \\
\hline & & 3.3 .1 & 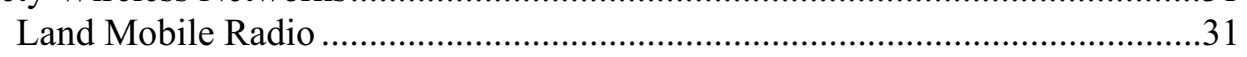 \\
\hline & & 3.3 .2 & 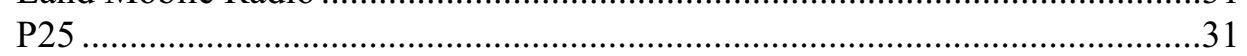 \\
\hline & & 3.3 .3 & TETRA ....................................... \\
\hline & 3.4 & Intero & bility Problems and Solutions \\
\hline & & 3.4 .1 & Network Patch Interconnect Solutions \\
\hline & & 3.4 .2 & IP Core \\
\hline & & 3.4 .3 & 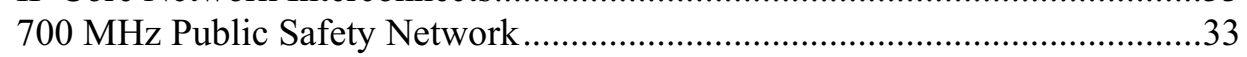 \\
\hline & & 3.4 .4 & Audio Gateways \\
\hline & & 3.4 .5 & 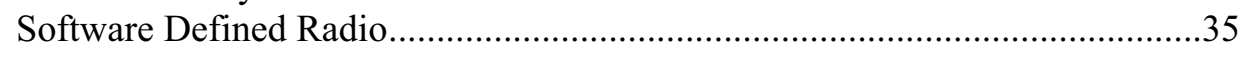 \\
\hline & & 3.4 .6 & 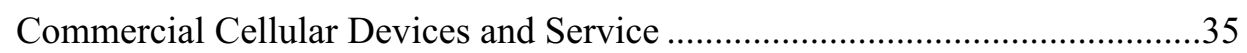 \\
\hline & 3.5 & Emers & 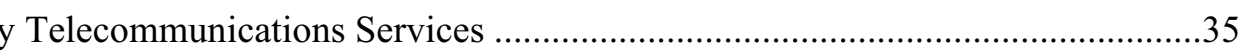 \\
\hline & 3.6 & Mun & NiFi Networks \\
\hline & 3.7 & Sumn & 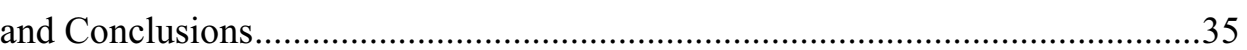 \\
\hline & Cat: & ophic $\mathrm{E}$ & 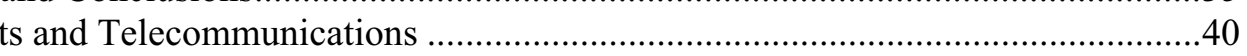 \\
\hline & 4.1 & Emers & 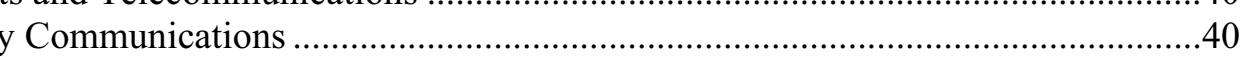 \\
\hline & & 4.1 .1 & 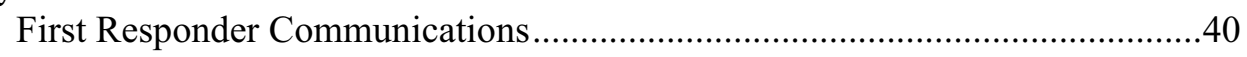 \\
\hline & & 4.1 .2 & Command and Control...................... \\
\hline & & 4.1 .3 & Critical Infrastructure Restoration ................. \\
\hline & 4.2 & Cellul & ommunication Outage: Hurricane Katrina........... \\
\hline & & 4.2 .1 & 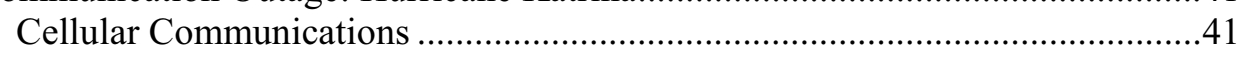 \\
\hline & 4.3 & $911 \mathrm{D}$ & 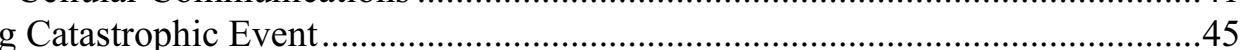 \\
\hline & & 4.3 .1 & 911 Call Traffic Patterns \\
\hline & & 4.3.2 & 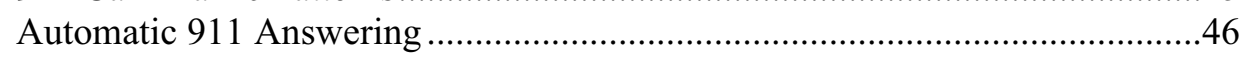 \\
\hline & & 4.3 .3 & 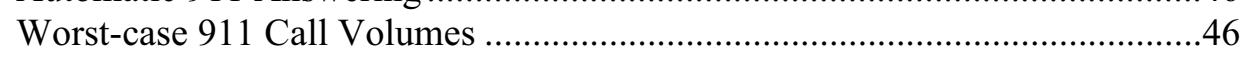 \\
\hline
\end{tabular}




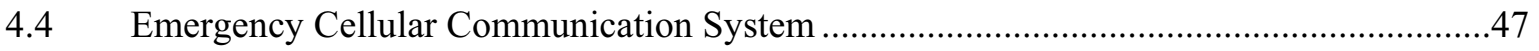

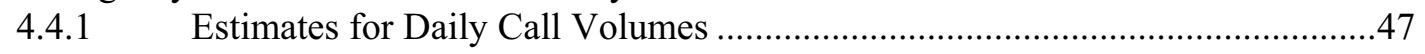

Estimation of Daily Maximum Load ........................................................... 48

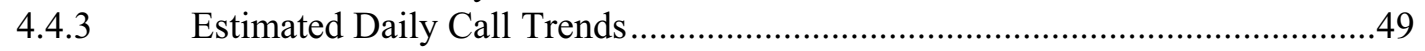

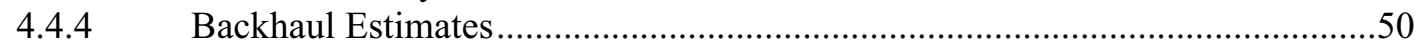

4.5 Estimates for an Airborne Wireless Data Network ......................................................51

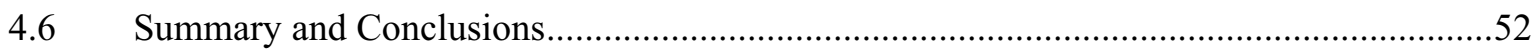

$5 \quad$ Options for Architectures and Restoration Techniques ......................................................56

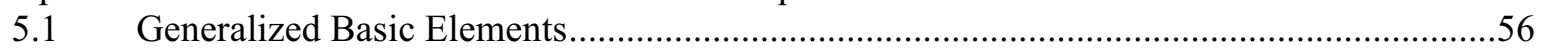

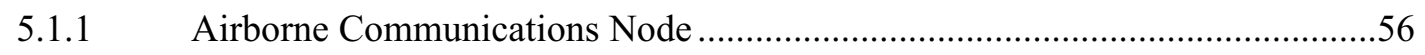

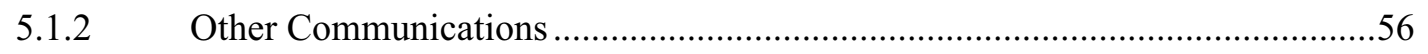

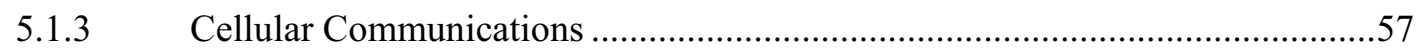

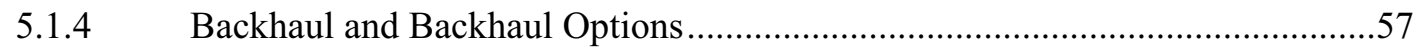

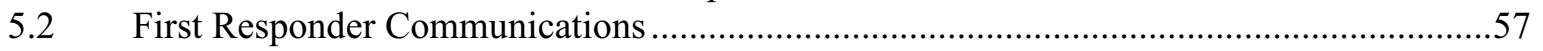

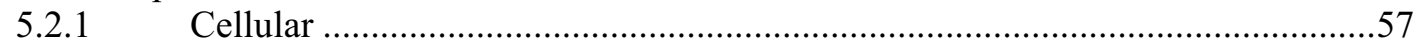

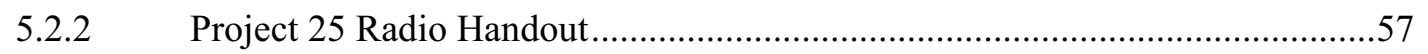

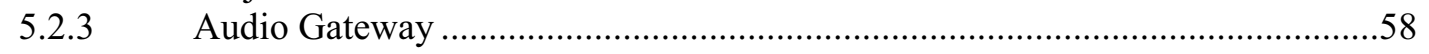

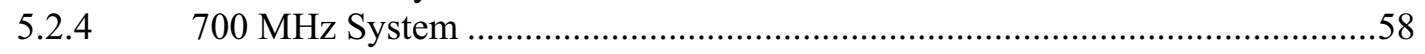

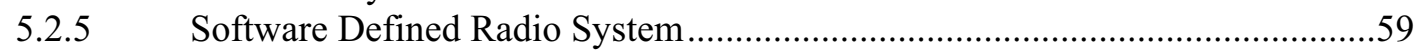

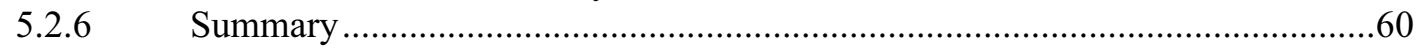

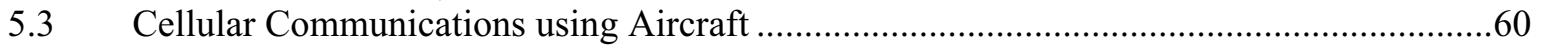

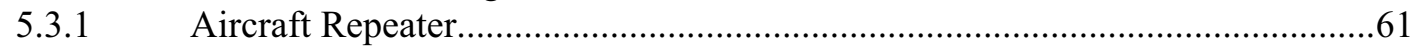

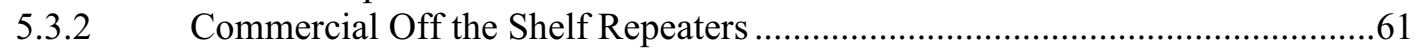

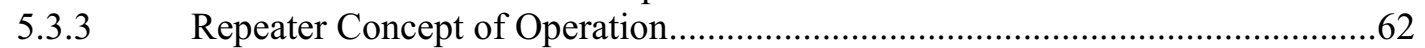

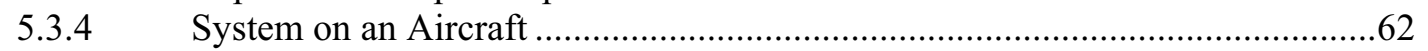

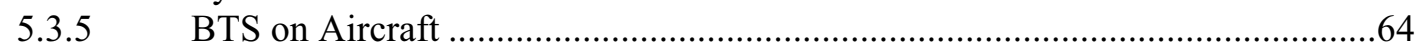

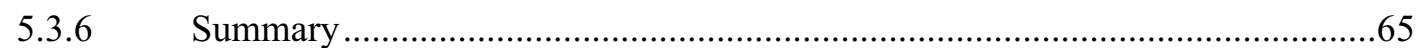

$5.4 \quad$ Additional Emergency Communications Capabilities ................................................66

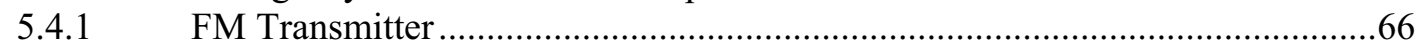

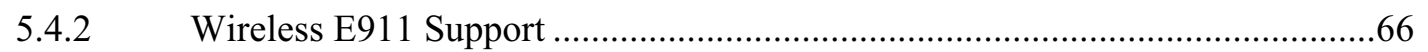

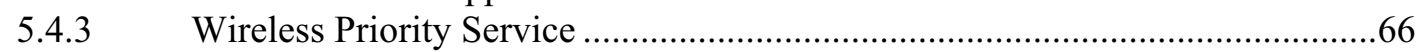

5.4.4 Emergency Cellular Broadcast ........................................................66

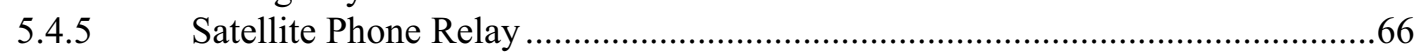

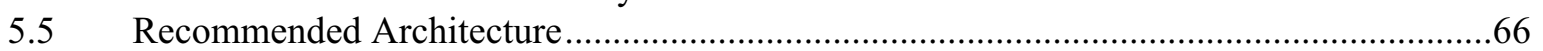

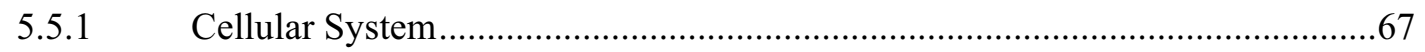

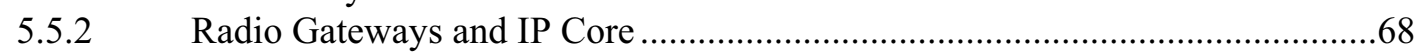

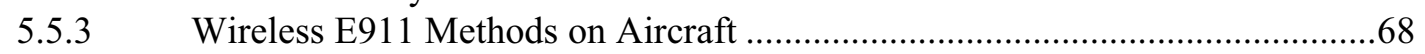

5.5.4 911 Auto Server, Remote PSAP and Emergency Operations Center .................69

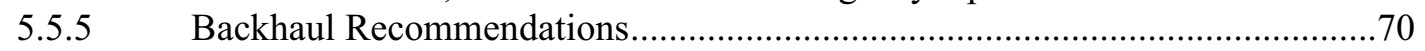

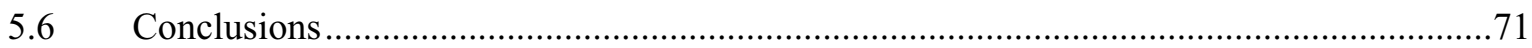

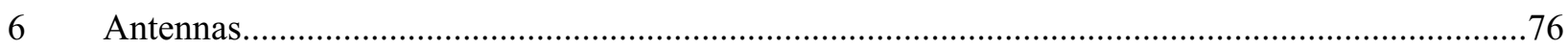

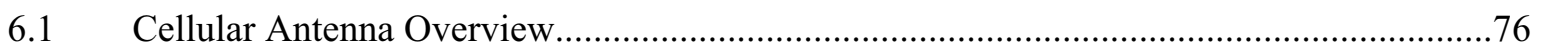

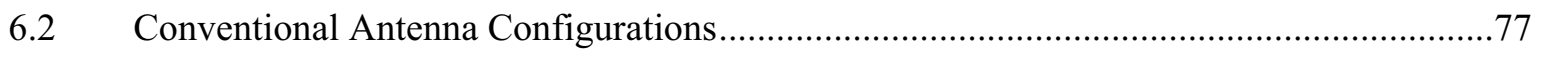

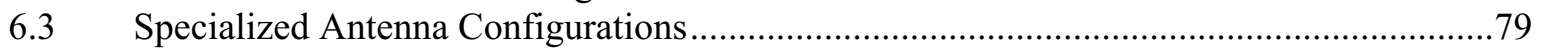

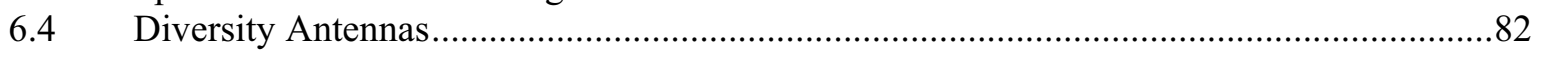

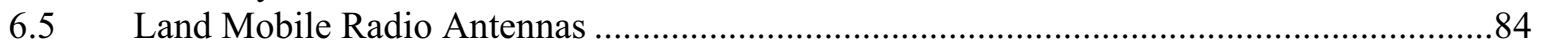

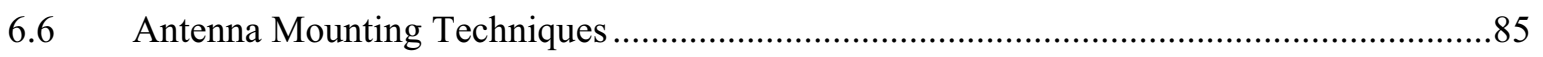

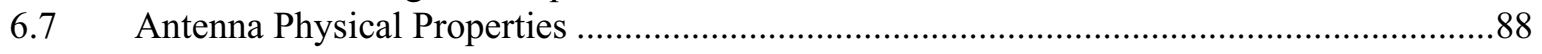

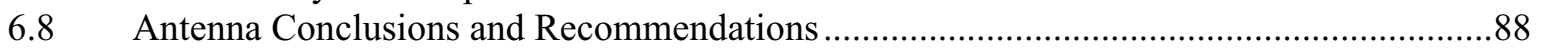




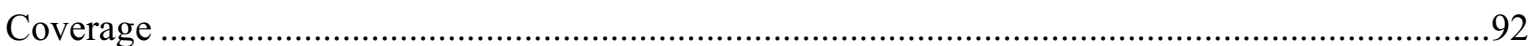

7.1 Impacts of Changing Flight Characteristics on the Coverage Model ..............................92

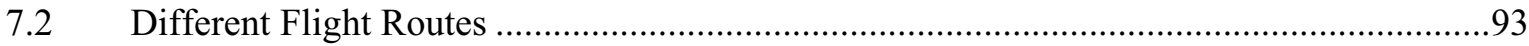

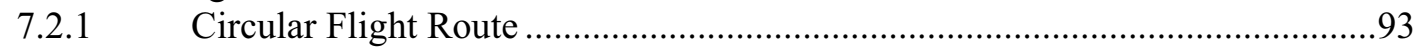

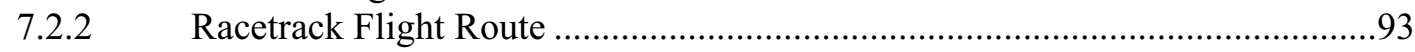

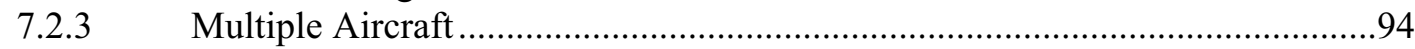

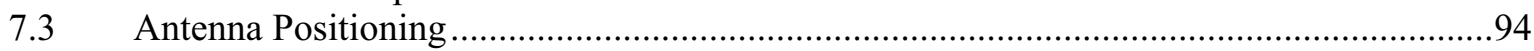

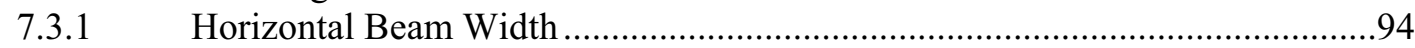

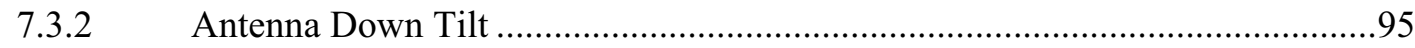

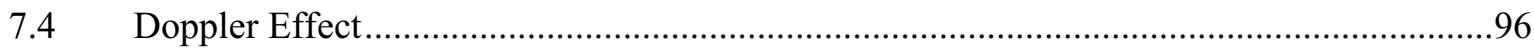

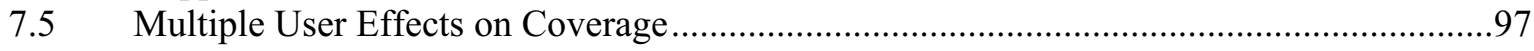

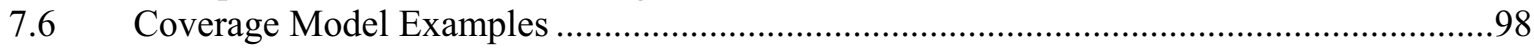

7.6.1 Circular Single Sector Coverage Model ......................................................... 98

7.6.2 Multi Sector Coverage Model .....................................................................101

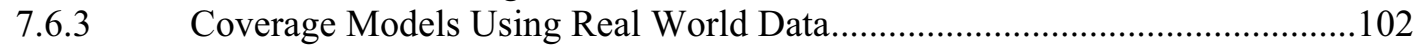

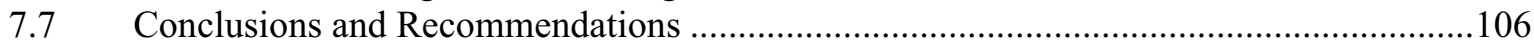

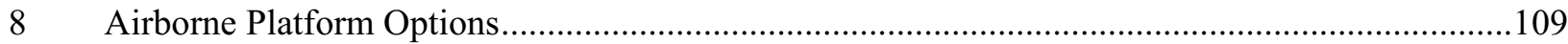

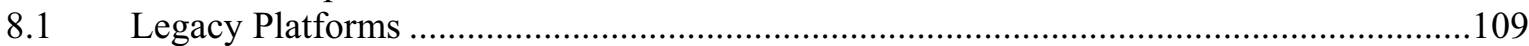

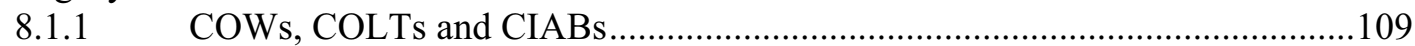

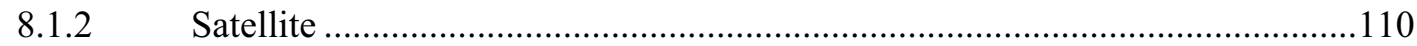

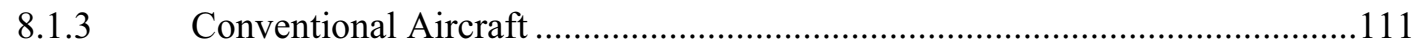

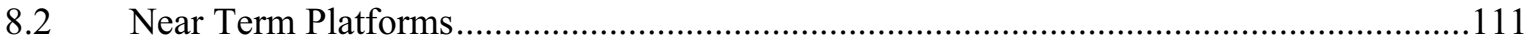

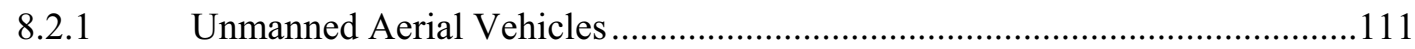

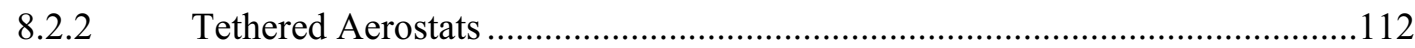

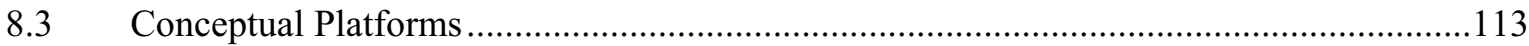

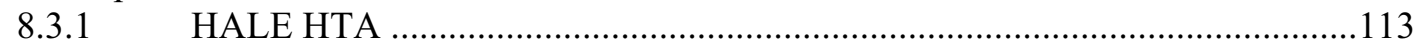

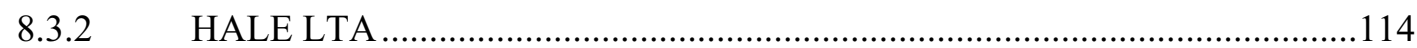

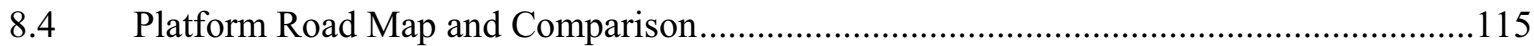

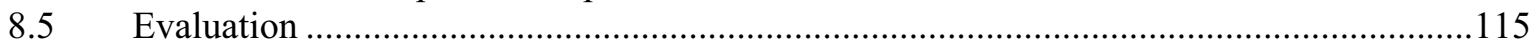

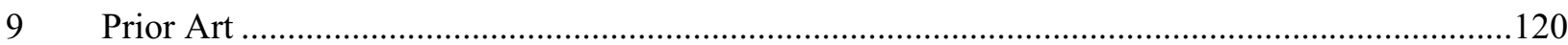

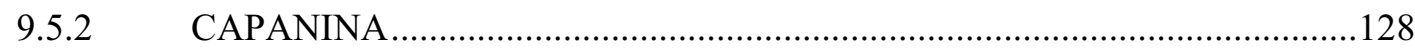

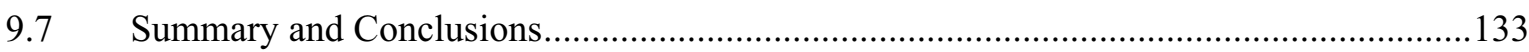

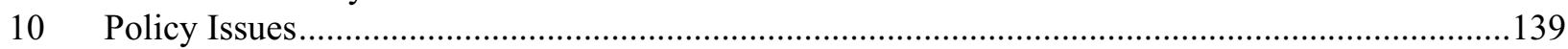

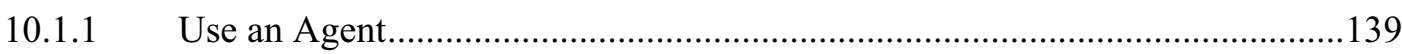

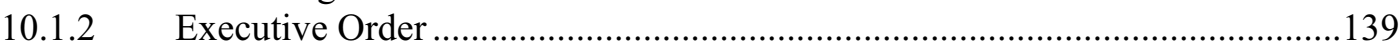

10.1.3 Space Division Multiplexing and Gap Filling .........................................139

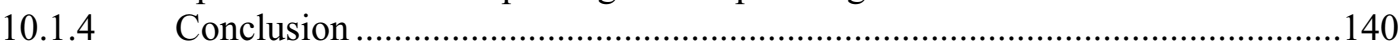

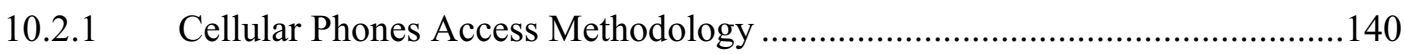

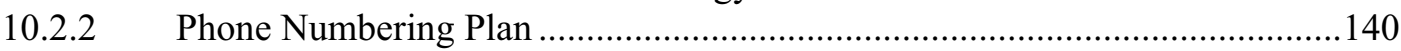

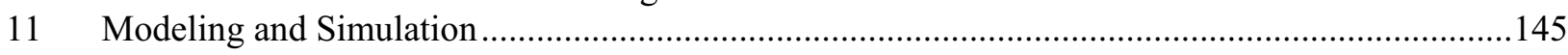

11.1 Electrical and Telecommunications Interdependencies .................................................145

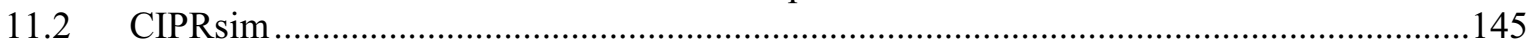

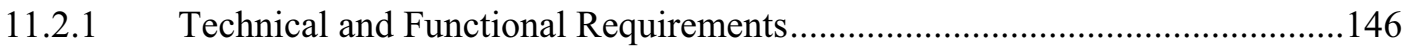

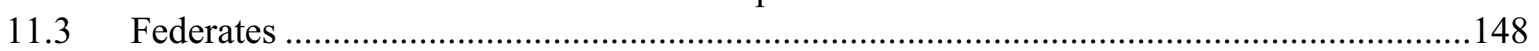

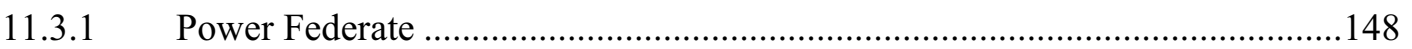

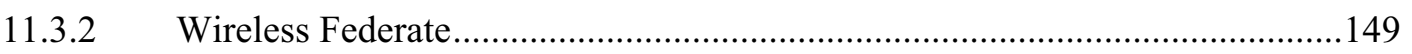

11.3.3 Hazard and Damage Assessment Federate ..............................................151

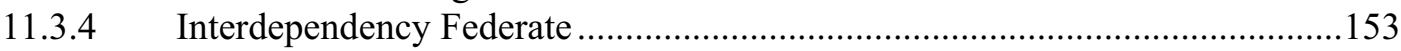

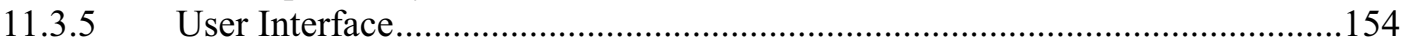




\section{TABLE OF FIGURES}

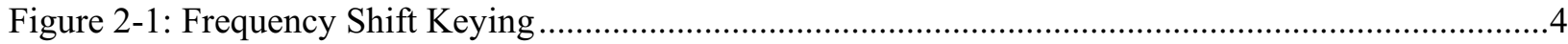

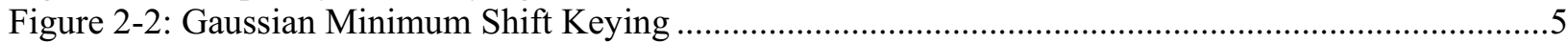

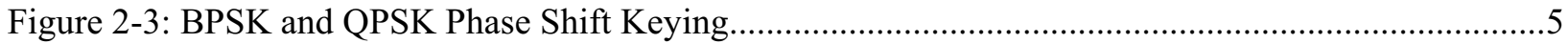

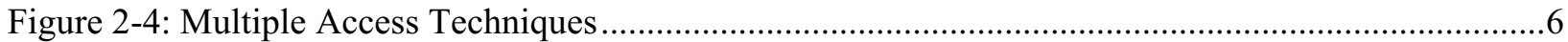

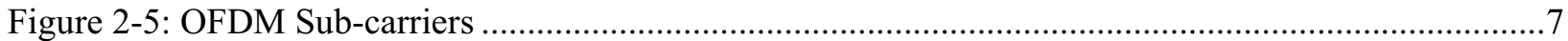

Figure 2-6: as Orthogonal Frequency Division Multiple Access Techniques.........................................

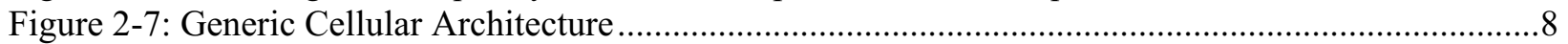

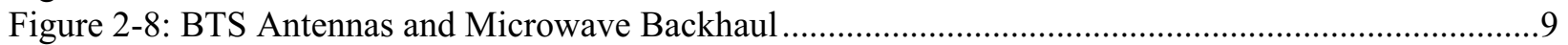

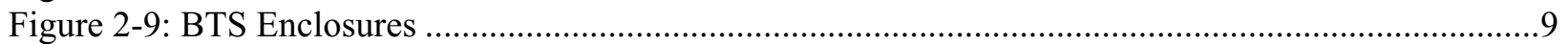

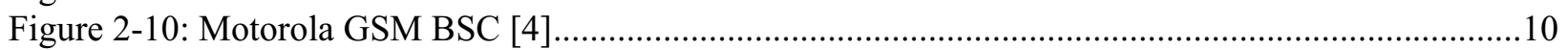

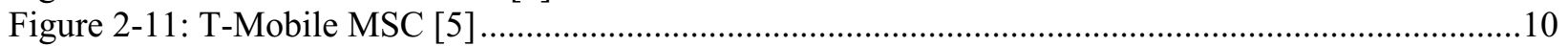

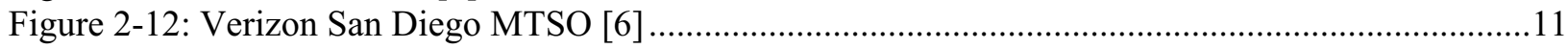

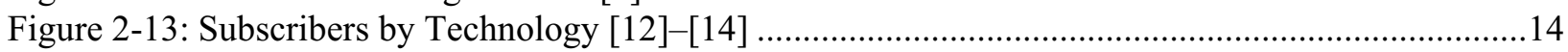

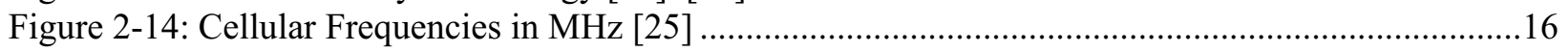

Figure 2-15: Cellular and PCS Frequency Blocks in MHz [26] ...................................................16

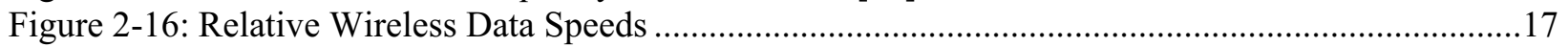

Figure 2-17: WiMAX Backhaul Supporting WiFi Hotspots [28] ........................................................18

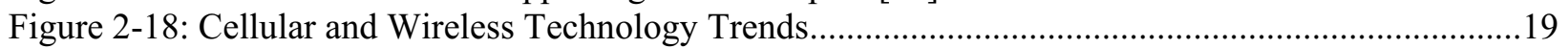

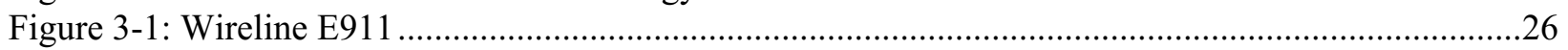

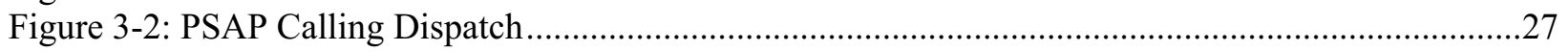

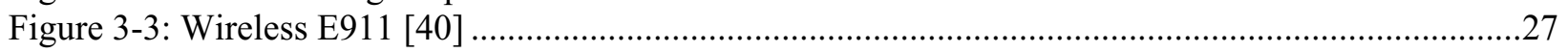

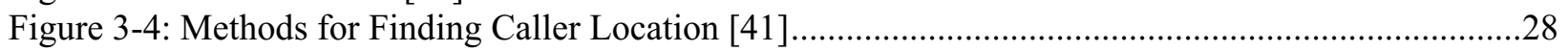

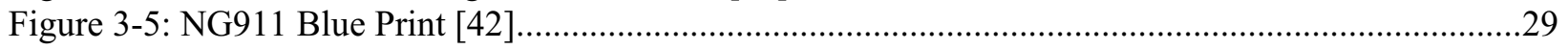

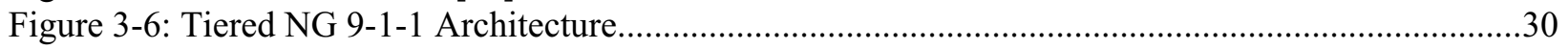

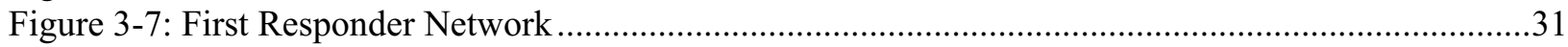

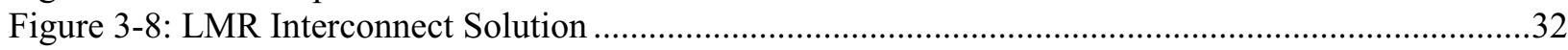

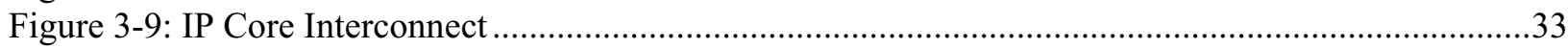

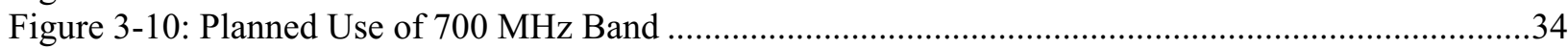

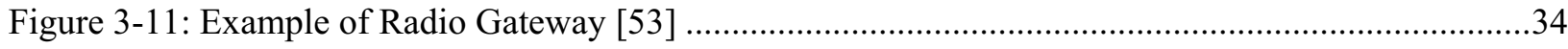

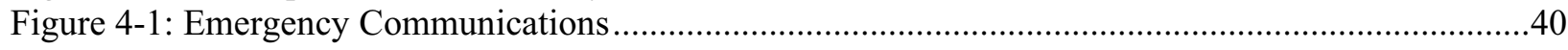

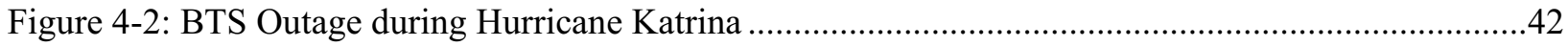

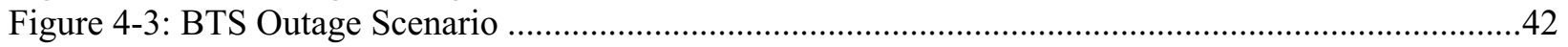

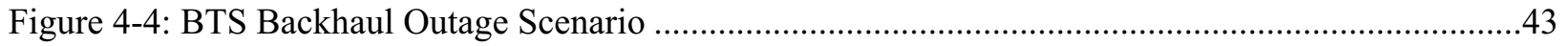

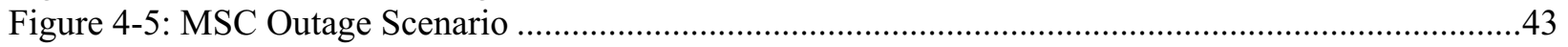

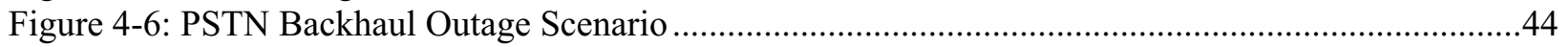

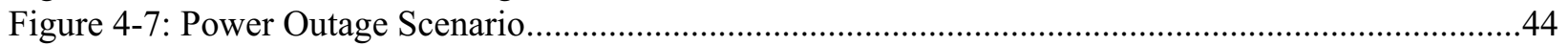

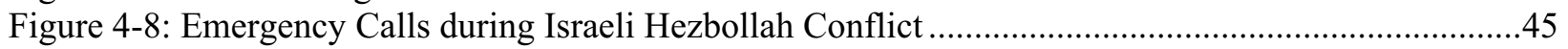

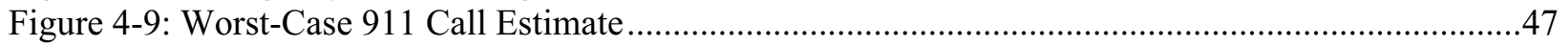

Figure 4-10: Estimated Daily Call Volumes .........................................................................................48

Figure 4-11: ACN and Terrestrial Assets Effectiveness Windows ........................................................49

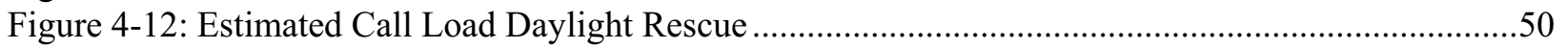

Figure 4-13: Estimated Call Load Continuous Rescue...........................................................................50

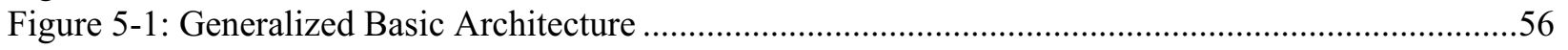

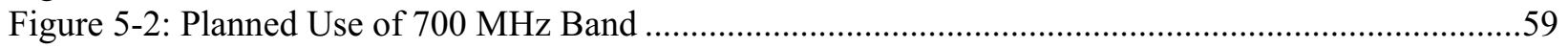

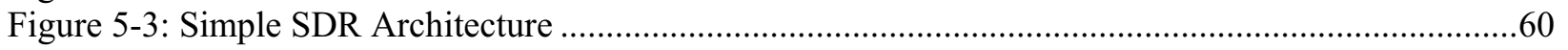

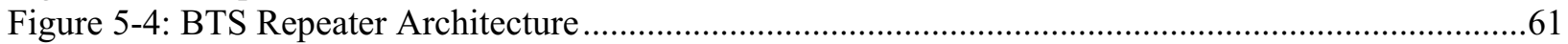

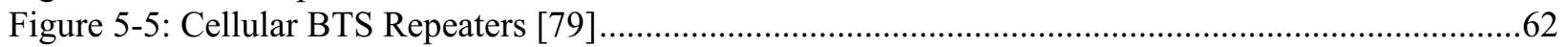

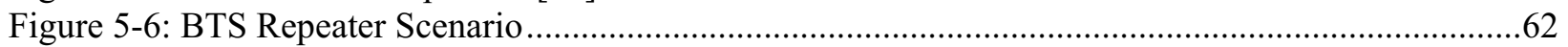

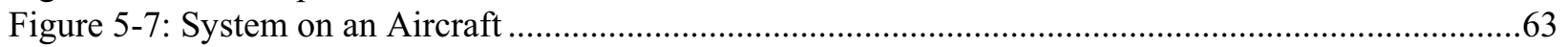

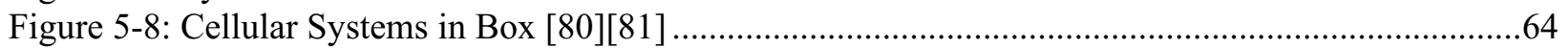




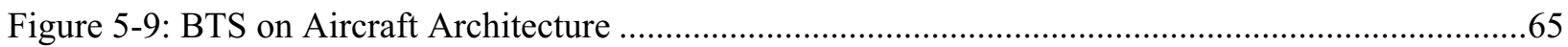

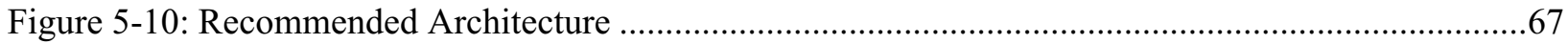

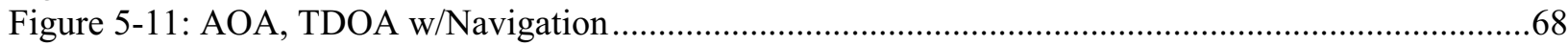

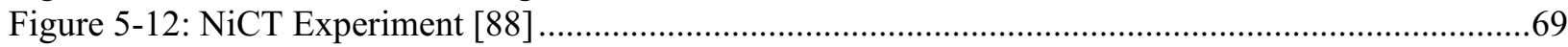

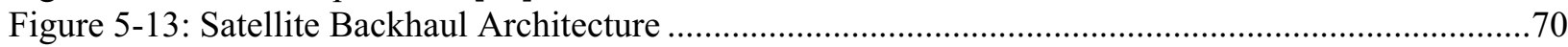

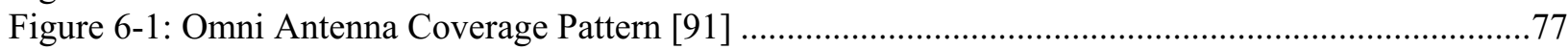

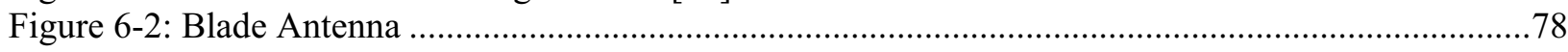

Figure 6-3: Three (Left Figure) And Six (Right Figure) Sector Configurations ....................................78

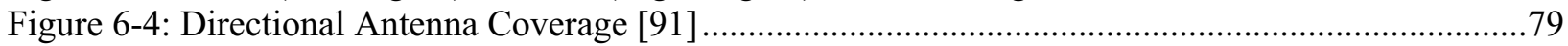

Figure 6-5: Switched Beam Smart Antenna Coverage Area (Omni) [91] ...............................................8

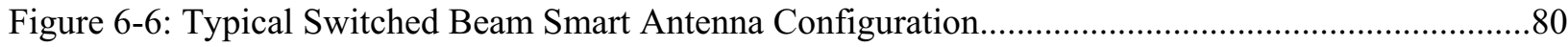

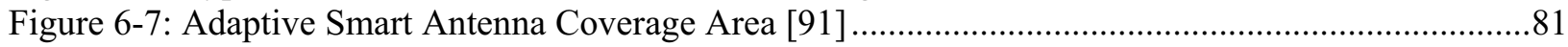

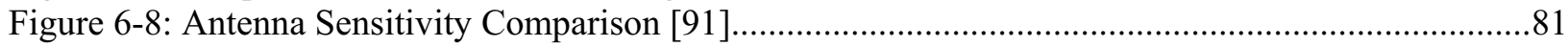

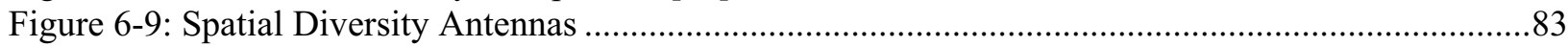

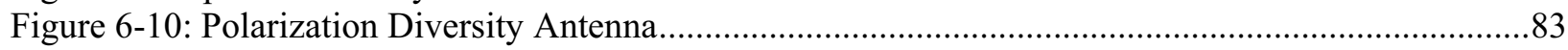

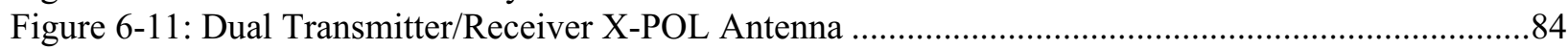

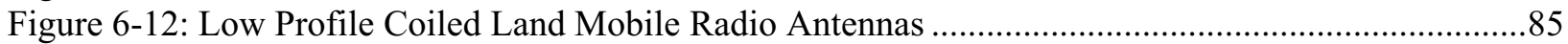

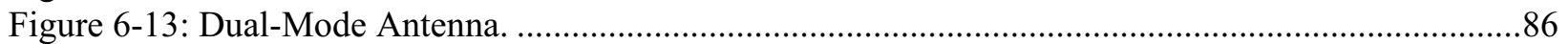

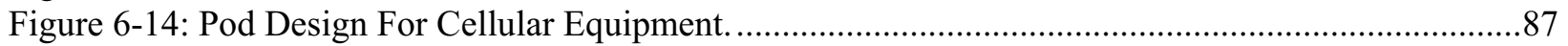

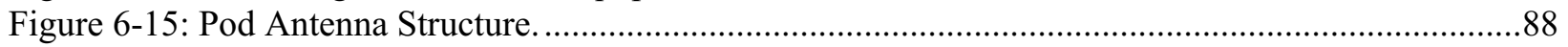

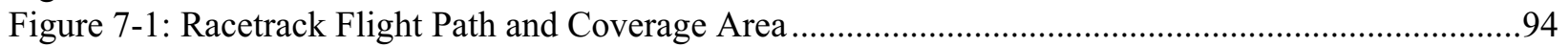

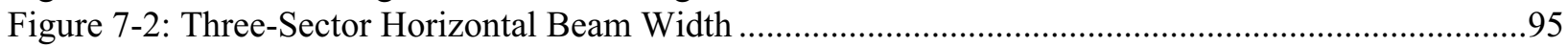

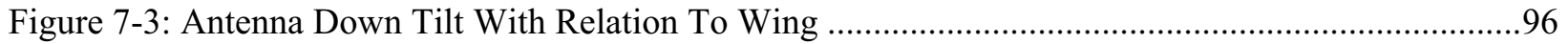

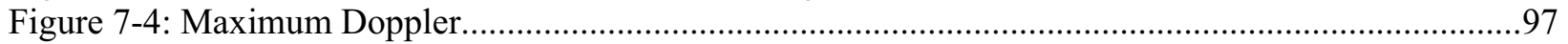

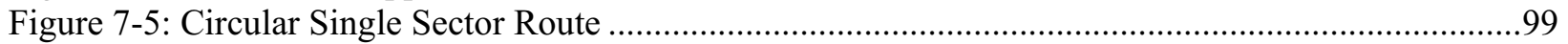

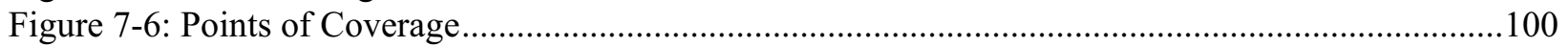

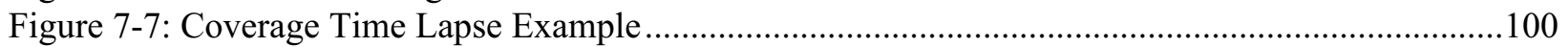

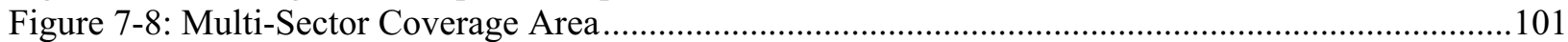

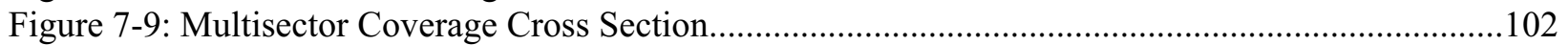

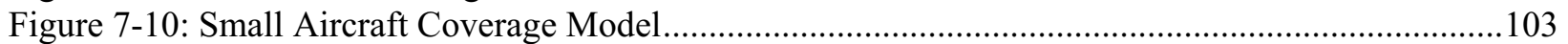

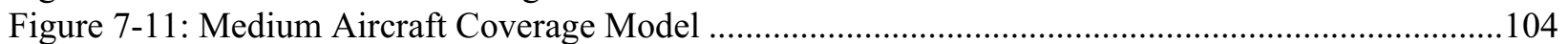

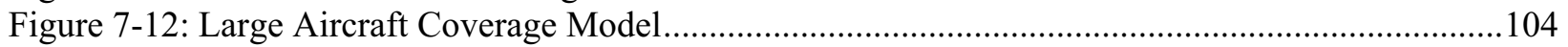

Figure 7-13: Unmanned Aerial System Coverage Model .................................................................. 105

Figure 8-1: CIAB (top left), COLT (top right), satCOLT (bottom left), and COW (bottom right) ..........109

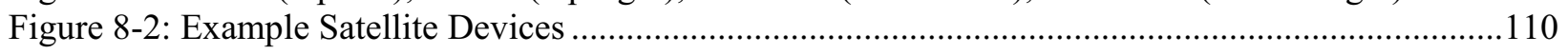

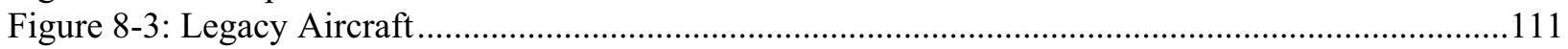

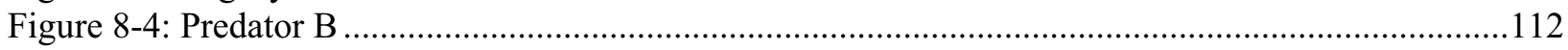

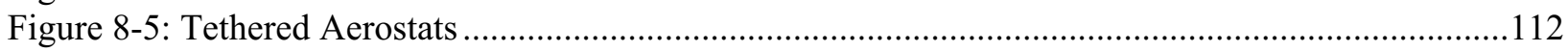

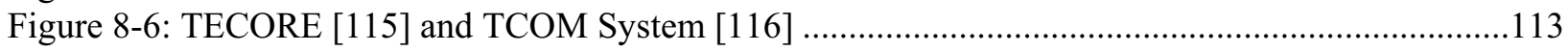

Figure 8-7: High Altitude Long Endurance Heavier than Air Platforms (HALE HTA) .........................113

Figure 8-8: High Altitude Long Endurance Lighter than Air (HALE LTA) Platforms ...........................114

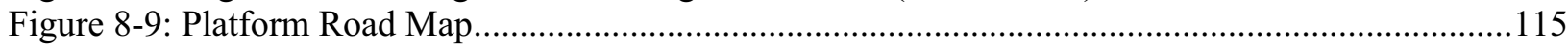

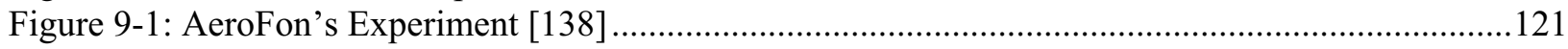

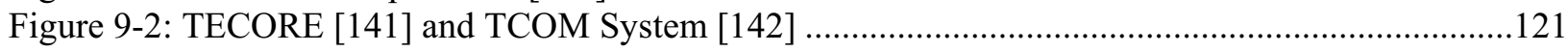

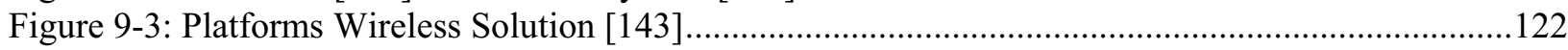

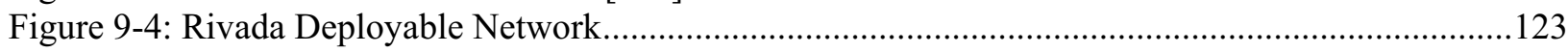

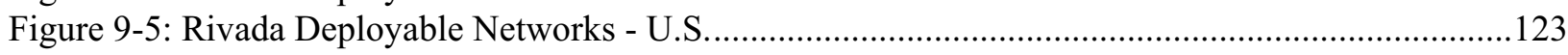

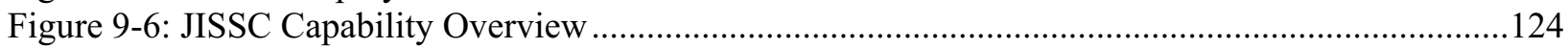

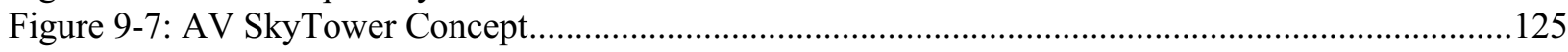

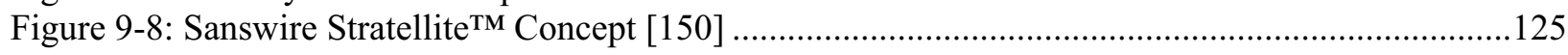

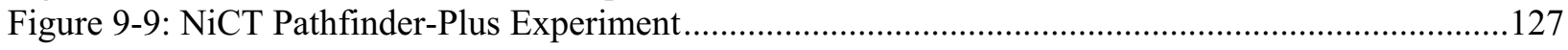

Figure 9-10: NiCT, Global Observer Prototype Experiment................................................................128 


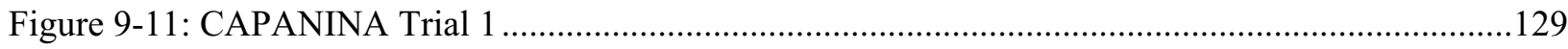

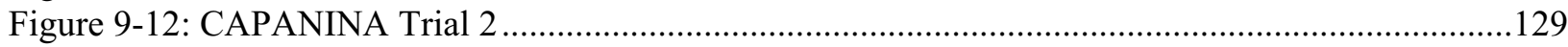

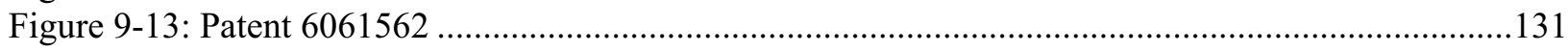

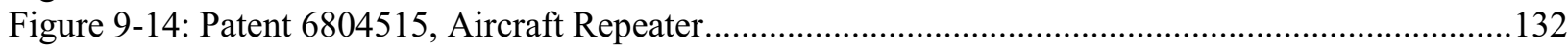

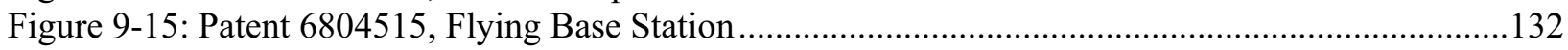

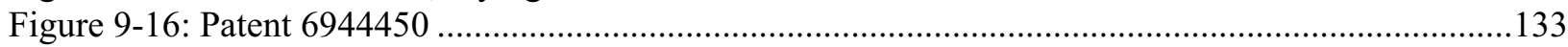

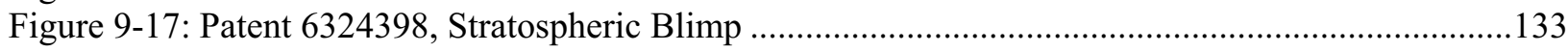

Figure 10-1: Space Division Multiplexing using beam forming antennas. ..........................................140

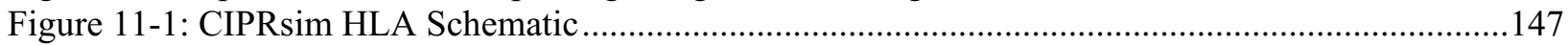

Figure 11-2: Wireless Coverage Polygons for INL Scenario .............................................................. 150

Figure 11-3: Example Wind Speeds and Storm Surge due to Hurricane ..............................................151

Figure 11-4: Asset States; Green-Operational, Yellow-Non-Operational, Red-Damaged........................152

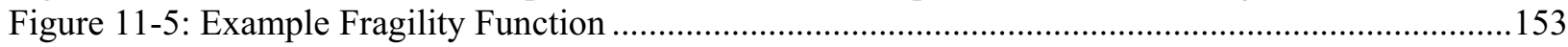

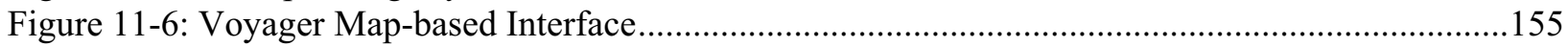

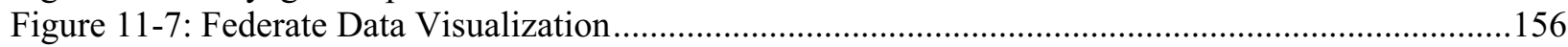

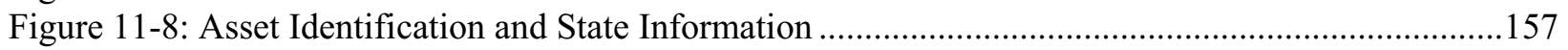

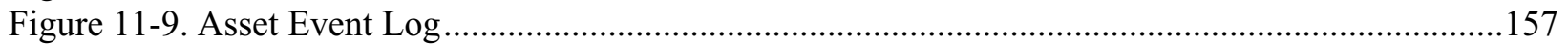

Figure 11-10: ACN Continuous and Instantaneous Coverage Areas ................................................158

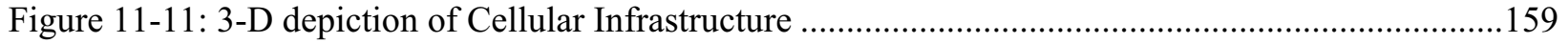

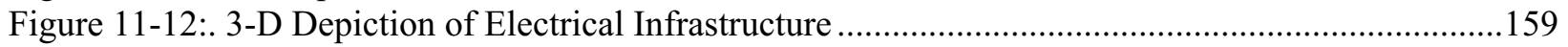

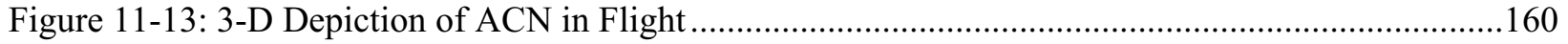




\section{TABLE OF TABLES}

Table 2-1: CDMA Technologies ..... 13

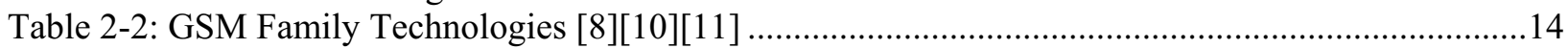

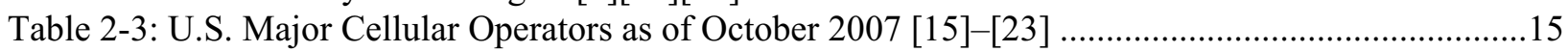

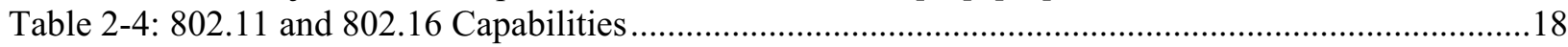

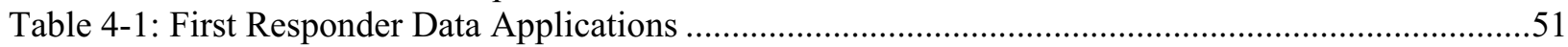

Table 5-1: Relative Comparison of First Responder Systems for ACN …….........................................60

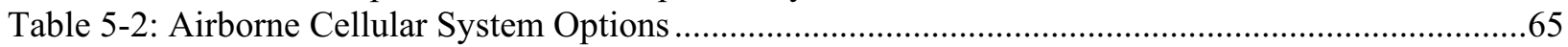

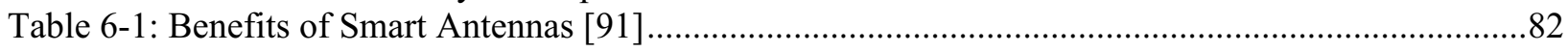

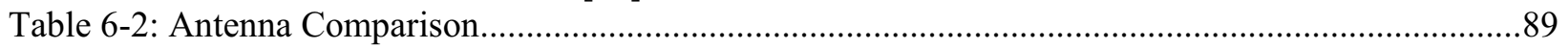

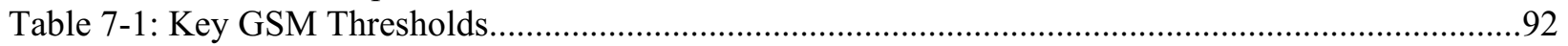

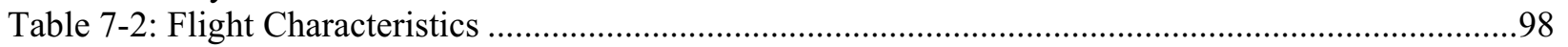

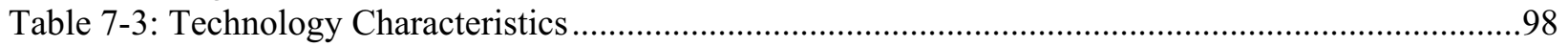

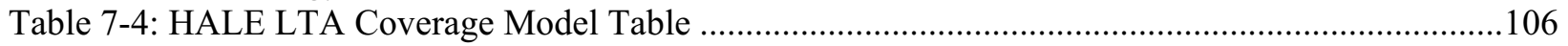

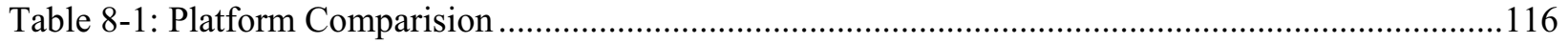




\section{Introduction}

\subsection{Overview}

In the event of a catastrophic event (i.e., natural or manmade incident, including terrorism, which results in extraordinary levels of mass casualties, damage, or disruption), access to reliable and enduring commercial communication systems is critical. Hurricane Katrina provided a recent example of the need to ensure communications during a national emergency. To ensure that communication demands are met during these critical times, the Idaho National Laboratory (INL), under the guidance of the United States Strategic Command has studied infrastructure issues, concerns, and vulnerabilities associated with the establishment of emergency wireless communications using a suite of equipment embedded on an aircraft. The capabilities provided in this study could provide wireless communications in an emergency until public/commercial nodes can be systematically restored.

\subsection{Objectives}

The objectives of this report are to:

- Analyze wireless communication outages due to a persistent man-made or natural catastrophic event

- Analyze how to provide critical communications services upon the occurrence of a catastrophic event

- Examine different restoration techniques using an airborne platform to restore wireless communications after the catastrophic event

- Analyze potential architectures for each of the airborne platform restoration techniques

- Recommend an architecture and technique for the airborne platform to be used after the catastrophic event

- Analyze antennas, antenna parameters as well as coverage patterns using aircraft

- Research Commercial Off-the-Shelf(COT) components and providers who could build a functional prototype of the recommended architecture

- Outline how the airborne platform might be used and discuss the policies and organizations that would need to participate in developing them.

\subsection{Organization}

\subsubsection{Wireless Networks}

The reader is assumed to be a wireless phone or application user and understands the applications, but not necessarily the infrastructure required to create a network. The section titled Wireless Networks provides the reader with a basic understanding of cellular and wireless network architectures. This section also explains the basic business environment of the cellular market and provides some predictions and trends of wireless technologies.

\subsubsection{Emergency Communications}

How a 911 emergency call makes its way to first responders is somewhat of an esoteric process. The purpose of this section is to explain how E911 and wireless E911 networks function as well as the plans for the Next Generation 911 networks, the functions of dispatch, and the different types of first responder communications. In addition, the challenge of interoperability is explained as well as some proposed solutions.

\subsubsection{Catastrophic Events and Telecommunications}

Catastrophic events or large disasters have specific unique effects on communications networks. The purpose of this section is to define those effects. Additionally, this section also quantifies worst-case scenarios in terms of emergency telecommunications system. 


\subsubsection{Options for Architectures and Restoration Techniques}

The purpose of this section is to define the different architectures, components and techniques used to provide critical communications from an Airborne Communications Node (ACN). It should be used to provide basic analysis for the different architecture possibilities while listing comparative advantages and disadvantages of each. We begin defining the scope by referring to the generalized basic elements. These basic elements are then explained further by examining different options for using aircraft for restoration techniques, architectures for each technique, and potential COTS equipment for each. An ideal architecture is also proposed at the end of the section.

\subsubsection{Antennas}

The purpose of this section is to explain the variety of antennas used for cellular and LMR radios and explains the advantages, disadvantages and the capabilities of each. We compare different mounting techniques for antennas and how existing aircraft capabilities could be utilized. This section ends by giving a relative comparison of different types of antennas.

\subsubsection{Airborne Communications Node Coverage}

When contemplating the deployment of an Airborne Communications Node (ACN), there are several constraints that should be considered. Physical constraints such as aircrew and aircraft durability, Doppler limitations of the communications technologies and the relationship to the speed of the aircraft, and antenna positioning constraints are taken into account throughout this section of the report. Additionally, more abstract constraints (e.g., distant cell interference, radio frequency spill over, and individual technology constraints [delay, Doppler Effect, and distance]), should be considered when planning the coverage capabilities of the ACN. This section discusses the variables that need to be considered when determining the coverage area of the ACN. Constraints (e.g., Doppler Effect, cell breathing, and path loss) are discussed along with the different types of coverage areas - either circular or racetrack.

\subsubsection{Platform Options}

There are several airborne platform options to consider when deploying an airborne communications node. All have different advantages and disadvantages when it comes to rapidly deployable emergency communications. These platforms include conventional aircraft, tethered aerostats, Unmanned Aerial Vehicles and high altitude aerostats. This section categorizes the different platforms and covers the strengths and weaknesses of each platform.

\subsubsection{Prior Art}

It is important to understand the state of the art and existing technologies available before acquiring new technological capabilities. The purpose of section is to provide a summarized description of existing commercial applications, experiments, research, and patents of airborne communications as uncovered in the research of this paper. The existing art in this field can be considered a starting point for examining vendors based on capabilities and an understanding of the current state and capabilities that have been demonstrated with technology.

\subsubsection{Policy Issues}

In researching communications via an airborne platform, many policy issues and questions arose out of conversations with cellular operators and government agencies. Although the main goal of the report is to report on the technical feasibility, the policy issues represent the greatest hurdle in providing emergency communications.

\subsubsection{Modeling and Simulation}

In order to assess the operational capabilities and limitations associated with an emergency airborne wireless communications system, an in-depth understanding of wireless communication, electrical infrastructure, and interdependency behaviors is critical. To address this need, Idaho National Laboratory developed the Critical 
Infrastructure Protection and Resilience Simulator (CIPRsim). CIPRsim provides a high-fidelity, dynamic modeling and simulation framework that illuminates greater understanding of the effects of disastrous events upon critical infrastructure components. 


\section{Wireless Networks}

\subsection{Introduction}

The purpose of section is to provide a fundamental understanding of the air interface, architectures and technology trends of wireless networks. Understanding air interface techniques such as modulation and channelization is essential to understand why wireless technologies are different. Despite differences in air interface standards, cellular architectures can be generalized into a generic architecture, which is essential in understanding how cellular networks operate and interoperate with other networks. Finally, understanding evolving technology trends is essential to understand future capabilities and business models of future wireless networks.

\subsection{The Air Interface}

The air interface is the communications link between two wireless devices. Several different techniques in digital modulation and channelization are used to form the air interface. To understand what makes each wireless technology unique it is important to understand the basics of each technique.

\subsubsection{Digital Modulation}

Digital modulation is the fundamental method by which data is transferred from one point to another using a wireless technology. Modulation is the method by which information is sent over the air using a carrier wave. Essentially, the carrier wave is manipulated in such a way that the receiver understands what the change in manipulation means. There are two basic forms of modulation that are used by most cellular and wireless technologies: Frequency Shift Keying (FSK) and Phase Shift Keying (PSK).

\subsubsection{Frequency Shift Keying}

Frequency Shift Keying is accomplished by manipulating the carrier wave's frequency to convey information. Figure 2-1, shows an example of FSK.

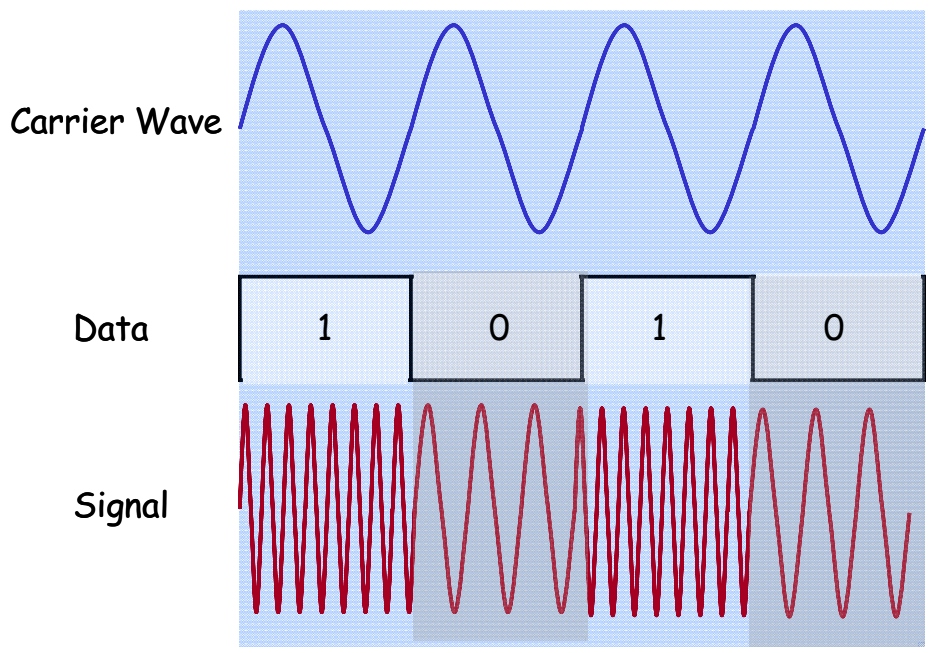

Figure 2-1: Frequency Shift Keying

In Figure 2-1 the sinusoidal carrier wave is used to transmit bits of data, in this case a " 1 " and " 0 ". To send this data the FSK modulator transmits the carrier wave with a higher frequency for a "1" and a lower frequency for a " 0 ." There are many variations of this technique. One commonly used variation of FSK in wireless technologies is called Gaussian Minimum Shift Keying (GMSK). In GMSK, the data pulses are converted into Gaussian shaped pulses before being sent to the modulator. Figure 2-2, shows an example of how this could work. 


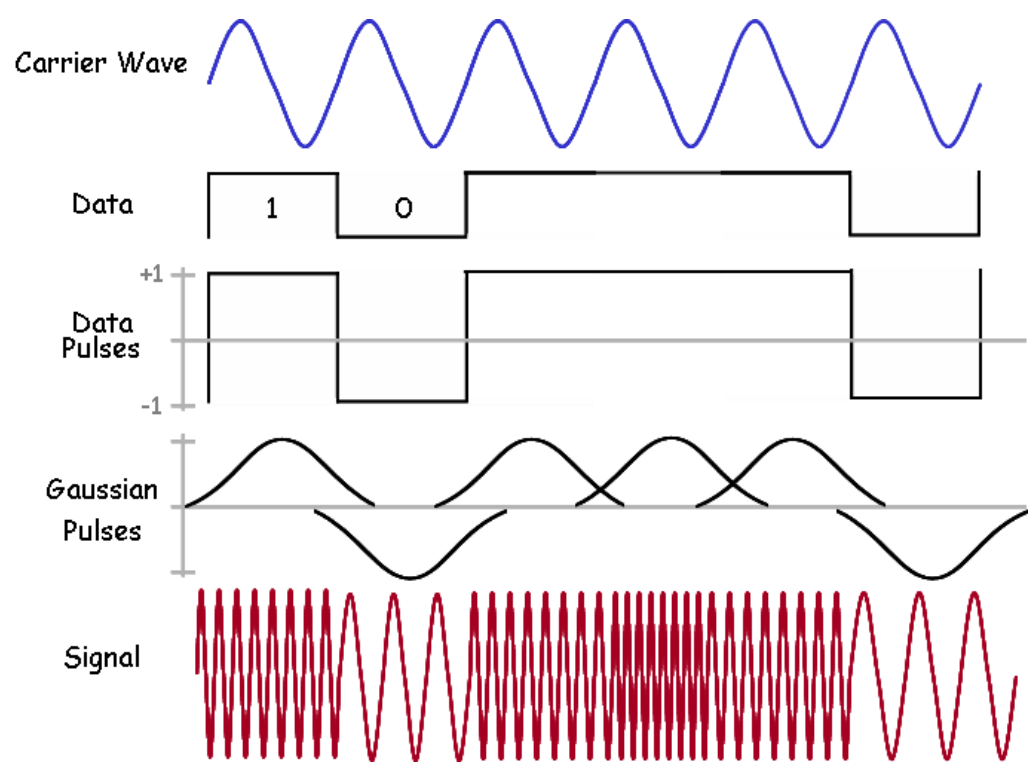

Figure 2-2: Gaussian Minimum Shift Keying

\subsubsection{Phase Shift Keying}

The other primary form of modulation is called Phase Shift Keying (PSK). This is done by manipulating the phase of the carrier wave to convey information. Figure 2-3 shows two different examples of PSK that are used: Binary Phase Shift Keying (BPSK) and Quadrature Phase Shift Keying (QPSK).

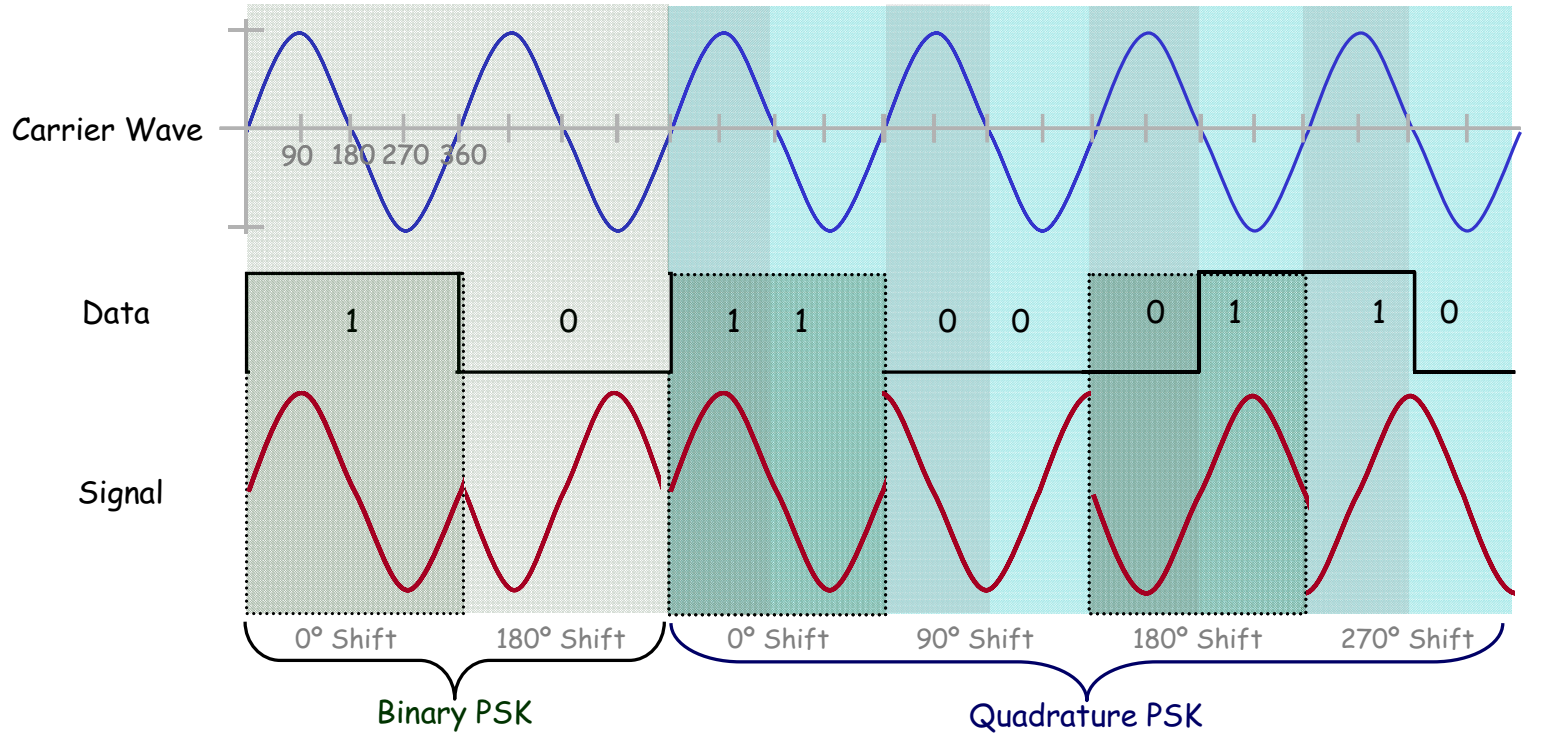

Figure 2-3: BPSK and QPSK Phase Shift Keying

In Figure 2-3, one period of a sine wave is used for the base signal. In order for BPSK to transmit a "1" the signal is transmitted as its base signal with no phase shift. To transmit a " 0 ", the signal is transmitted with a shift of $180^{\circ}$. As shown in Figure 2-3, QPSK uses shifts of $0^{\circ}, 90^{\circ}, 180^{\circ}$ and $270^{\circ}$ to transmit bit sequences. In QPSK, twice as many bits can be transmitted in a single modulation period. In the figure, transmission of the $0^{\circ}$ will be understood as "11" (two bits that are both "1"). A $90^{\circ}$ shift would be " 00 ", a $180^{\circ}$ shift as " 01 " and a 270 " shift as "10." New and future wireless technologies are beginning to use Quadrature Amplitude Modulation (QAM) which is an advanced PSK technique. QAM uses more granular shifts in phase and changes in signal amplitude to bit sequences of 16 bits or more in a single period. 


\subsubsection{Channelization and Multiple Access Techniques}

Multiple Access (MA) is used to describe how multiple channels or callers are able to share a segment of spectrum. This separation of different traffic sources is called channelization. These traffic sources could be either user traffic or signaling. The three most common multiple access techniques used in cellular technologies are Frequency Division Multiple Access (FDMA), Time Division Multiple Access (TDMA) and Code Division Multiple Access (CDMA).

FDMA allows MA by allowing each traffic source to have its own frequency channel. Figure 2-4: Multiple Access Techniques, shows an example of a hypothetical $\sim 4 \mathrm{MHz}$ bandwidth divided up evenly in four separate 1 $\mathrm{MHz}$ channels and separated by guard bands. (This figure and example is shown for explanation purposes only. Wireless technologies use very different bands and channel bandwidths) These channels allow multiple users or talk groups to use the same system by using their own frequency channel. This is common with early Land Mobile Radio systems and analog cellular systems. When early cellular systems became increasingly popular, the demand required more advanced MA techniques to accommodate more users in the same amount of spectrum.[1]

In response to the growing demand of cellular users, the TDMA technique was invented. TDMA channelization is done by dividing FDMA channels into different time slots. Figure 2-4, shows our four frequency channels divided into four different time slots and separated by time guard bands. In our example, the capacity was increased on each frequency channel. Each user channel uses a timeslot instead of one frequency channel. This made more efficient use of spectrum allowing more users onto the available segments of spectrum.

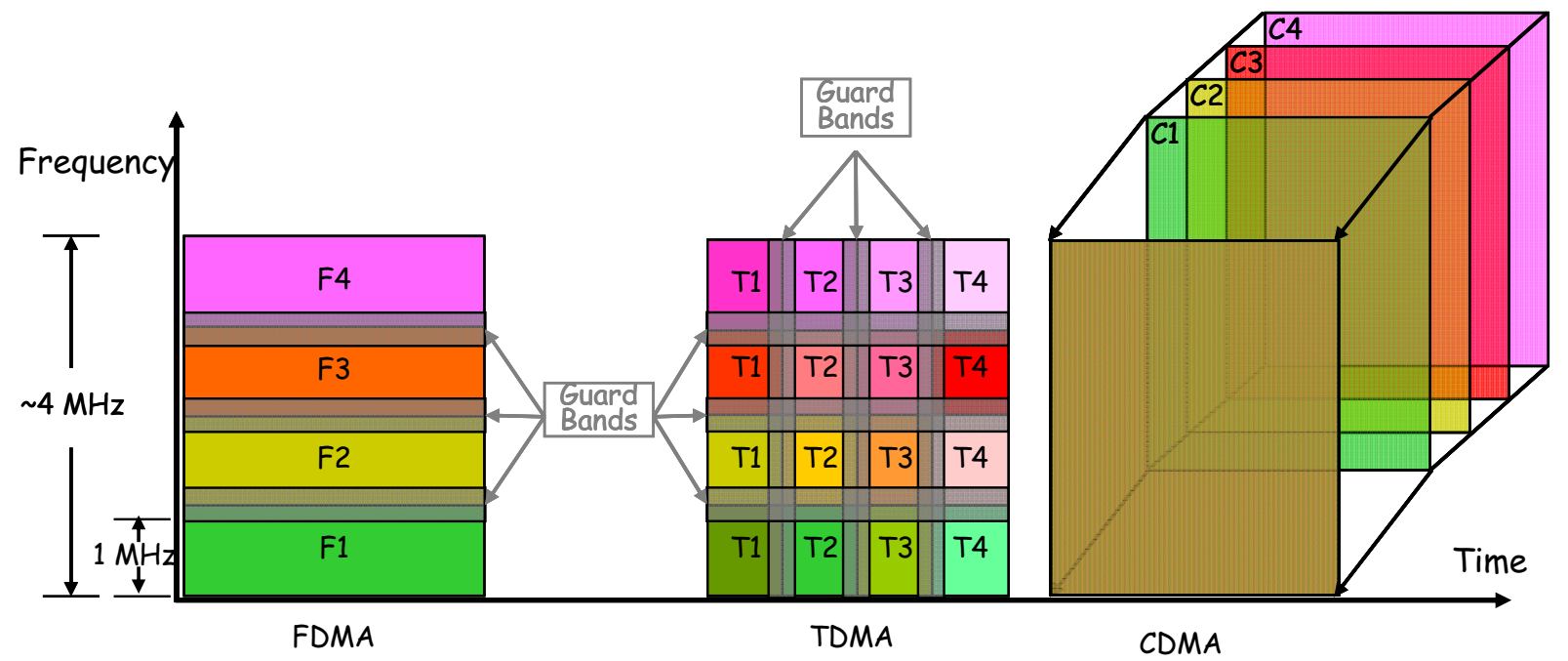

Figure 2-4: Multiple Access Techniques

CDMA was also invented for increasing capacity. Instead of using timeslots to separate out channels, CDMA used orthogonal digital codes. These digital codes enable the digital separation of each channel. The CDMA technique also uses a single wider transmission channel. Figure 2-4 right, shows a pictorial representation of CDMA using the entire $4 \mathrm{MHz}$ block. Each pane represents a code, indicated by C1, C2, C3, and C4. Each code in this example is represented by a specific color. If channels are transmitted together they form the forward brown pane. However, removing only the green from the image will yield and allow selection of a specific channel.

An audio representation of CDMA can also be thought of as many people in a room speaking 20 different languages. A native speaker of English would be able hear what another English speaker is saying over what the other 19 speakers in the room. However, as the number of speakers increase, or the number of panes, it becomes more difficult to identify an individual signal from the rest. As the noise in the room grows louder or the pane in the forefront becomes darker, the individual signals overwhelm each other. For mitigation of noise, CDMA technologies will use sophisticated power control algorithms to help control overall noise levels. 
In contrast with a single-access system, such as a two way radio system, where each user will use the same frequency for receiving and transmitting, MA systems allow for multiple channels. FDMA, TDMA, and CDMA are primary techniques used. These techniques are one of the main attributes which differentiates wireless technologies.

\subsubsection{Orthogonal Frequency-Division Multiplexing and Orthogonal Frequency Division Multiple Access}

Orthogonal Frequency-Division Multiplexing (OFDM) is another technique used to improve spectrum efficiency. OFDM uses multiple orthogonal sub-carriers which are spaced such that their harmonic frequencies effectively cancel out each other (Figure 2-5, left.) This will result in several distinct separated channels (Figure 2-5, right.) Several parallel bit streams are then transmitted on these narrow orthogonal sub-carriers simultaneously. [2] This greatly improves efficiency of spectrum use and data throughput.

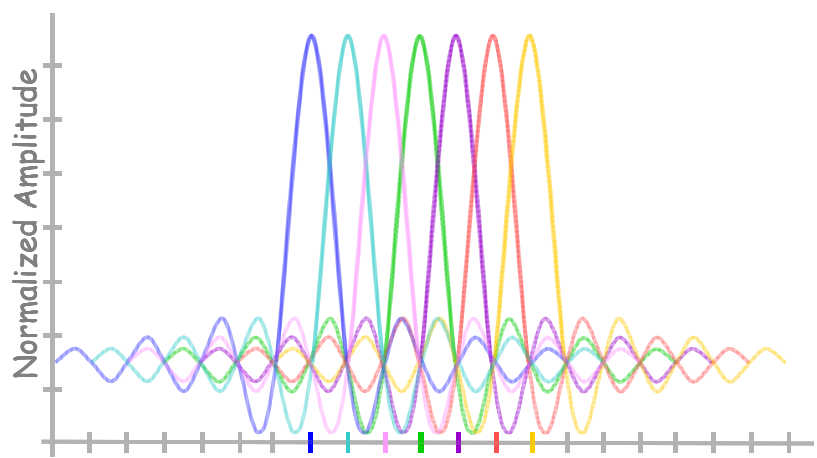

Normalized Frequency

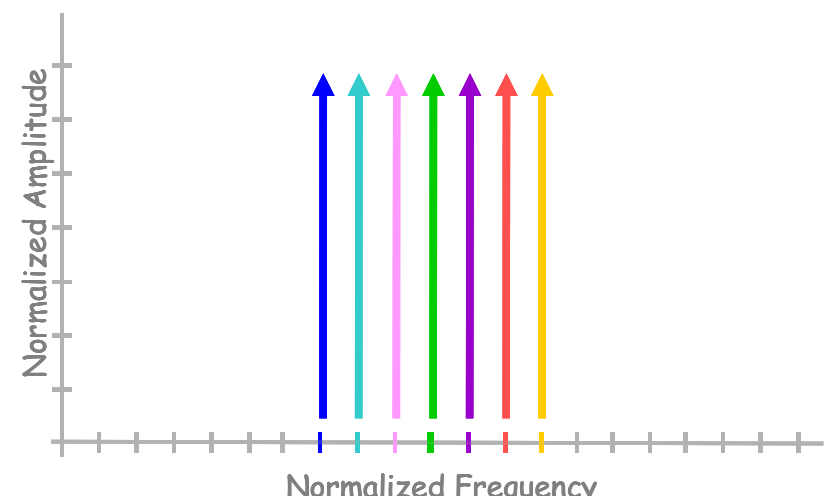

Normalized Frequency

Figure 2-5: OFDM Sub-carriers

OFDM can also be used as a MA technique known as Orthogonal Frequency Division Multiple Access (OFDMA). This technique is a combination of OFDM, TDMA and/or FDMA. This is much more efficient than the previous multiple access techniques because no guard bands are used for channelization. Figure 2-6 shows two simple examples of OFDMA. TDMA OFDMA will separate the different OFDM channels into timeslots and transmit them into their timeslots. FDMA OFDMA will use the different sub-carriers or sets of sub-carriers to form a channel. [3]

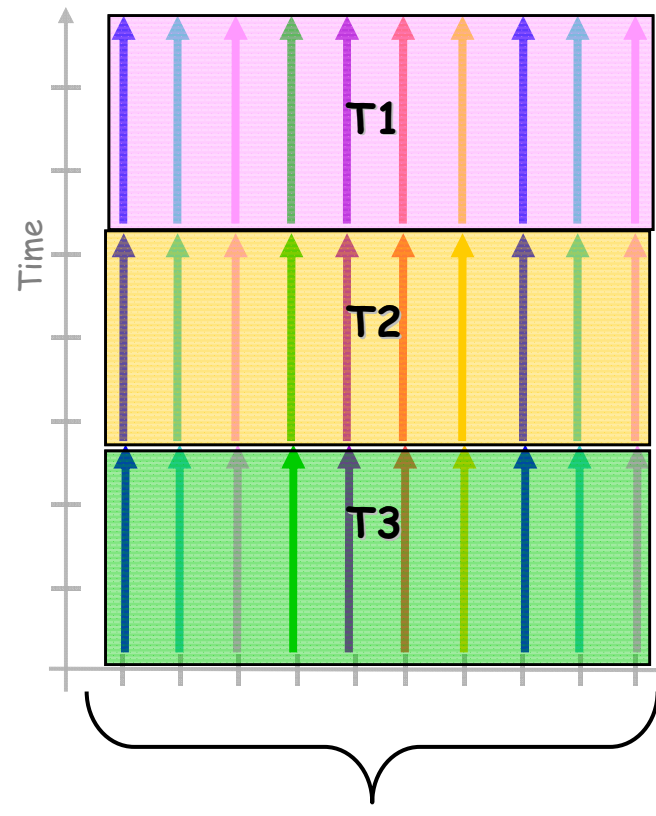

TDMA OFDMA

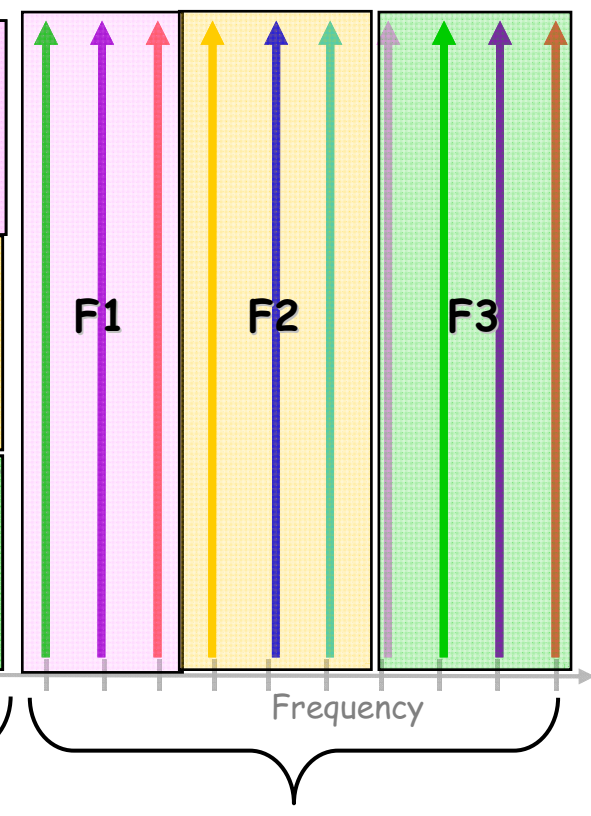

FDMA OFDMA

Figure 2-6: as Orthogonal Frequency Division Multiple Access Techniques 


\subsection{Cellular System Architectures}

Cellular architectures come in many variations depending on technology and vendor implementation. However, many different components of these cellular architectures can be generically grouped into logical network elements. These generic elements of a cellular architecture are shown in Figure 2-7: Generic Cellular Architecture. The generic network elements are the following: (from left to right in Figure 2-7):

- Mobile Subscriber (MS)

- Also known as a cell phone, mobile or access terminal (AT)

- Provides user access to the network

- $\quad$ Base Transceiver Station (BTS)

- Also known as "Base Station"

- Provides air interface to mobile

- Base Station Controller (BSC)

- Provides call control and mobility management

- Mobile Switching Center (MSC)

- Provides primary Connection into the Public Switched Telephone Network

- Provides high level call control between BSCs

- Public Switched Telephone Network (PSTN)

- Provides access to the traditional wire-line telephone network and other cellular networks

- Packet Data Node (PDN)

- Provides a route for packet data to and from the internet.

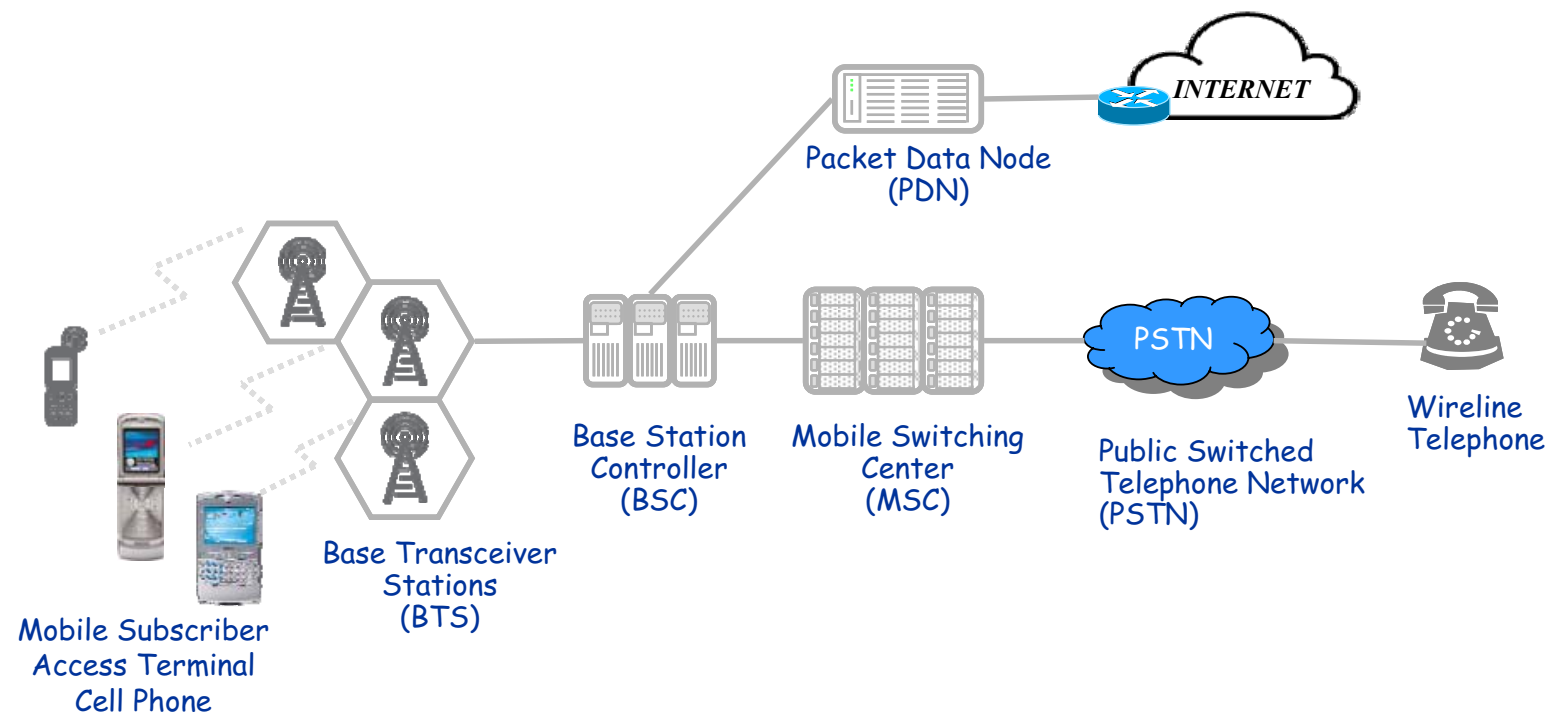

Figure 2-7: Generic Cellular Architecture

\subsubsection{Base Transceiver Station and Backhaul}

The Base Transceiver Station (BTS) primary function is to provide a Radio Frequency (RF) link to the MS. BTSs will provide generic channel elements which are used by the MS to access the network. An average site will support approximately 2000 simultaneous users; although, the number of supported simultaneous users per site depends on the traffic density and the geographic coverage area radiated by the BTS. This geographic coverage area is called a cell. Cell sizes can vary from a 10-mile (rural scenario) to 1/4-mile (urban scenario) radius. 
The BTS antennas can be found on top of monopoles, lattice towers or buildings (see Figure 2-8: BTS The antennas and towers can be identified by the triangular shaped antenna platform with multiple white antennas on each side of the triangular structure. At the base of the tower, enclosures containing the BTS radio equipment are usually present.
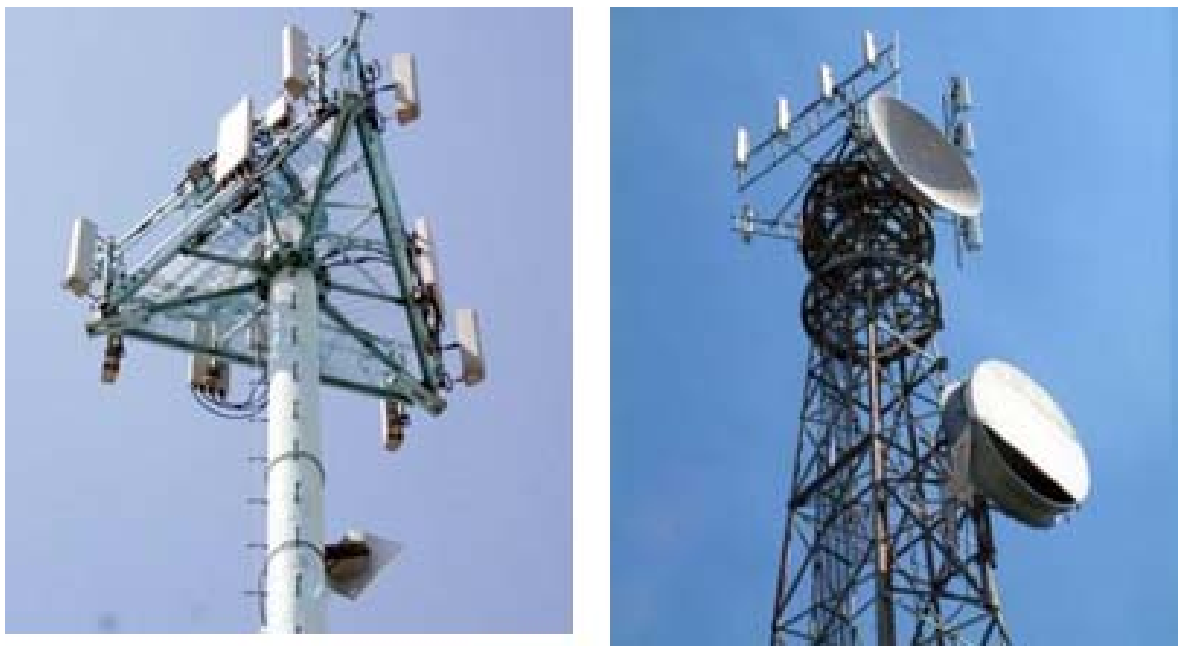

Figure 2-8: BTS Antennas and Microwave Backhaul

Facilities of the BTS enclosures can include: back up batteries, generators, air conditioning units, backhaul equipment as well as BTS transceivers. Backup power systems will automatically activate during a power outage and typically have a run time of 48 hours. In some cases, air conditioning is required for BTS equipment, specifically for BTS transceivers which can get hot due to the amount of power used for RF transmission. Additionally, connections to backhaul equipment allow signals from the BTS to travel back to the BSC. Figure 2-9 shows some examples of BTS enclosures the black cables (in sets of three) connect to the antennas.
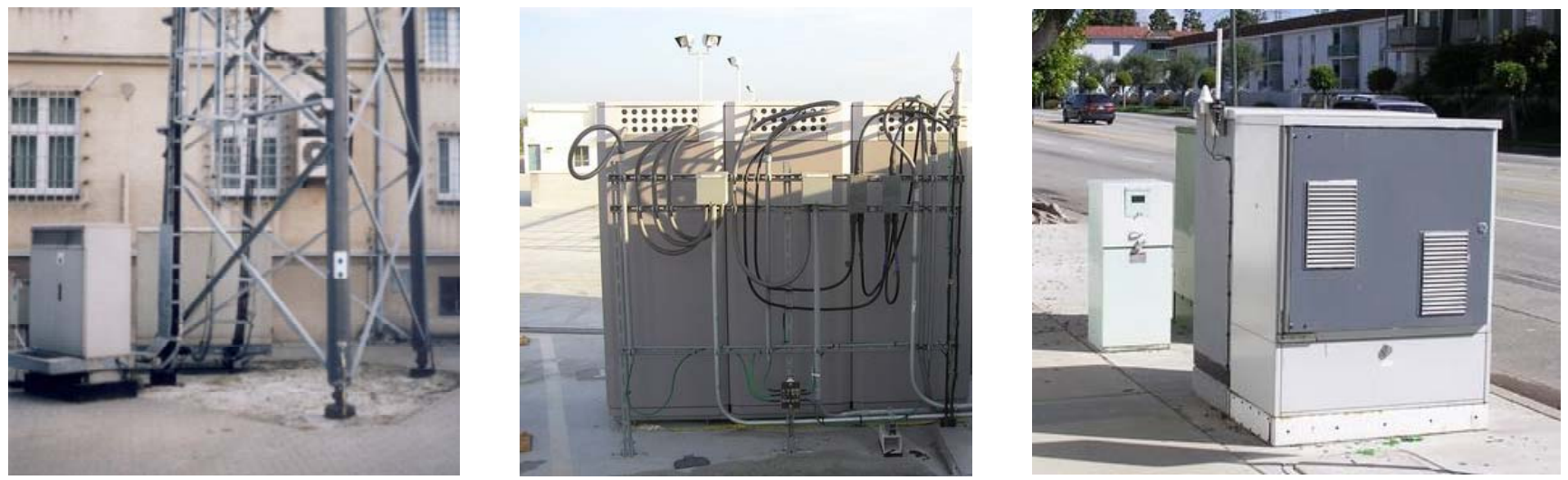

Figure 2-9: BTS Enclosures

Signals travel from the BTS to the BSC using what as backhaul links. Backhaul links are supported by a T1 connection to the BSC via backhaul equipment. T1s are used for logical separation of voice calls on a single transmission line. T1s used for the backhaul links could travel over fiber optics, copper wire or microwave for backhaul. Round microwave dishes, shown in Figure 2-8: BTS , are also typical along the tower or monopole for providing this Backhaul to the BSC. 


\subsubsection{Base Station Controller}

The Base Station Controller (BSC) is the brains of the BTS. A typical BSC could control up to 50 BTSs coordinating handoffs between BTSs, vocoding and processing resource requests for calls. Calls may be made from vehicles moving at high speeds; the BTSs will need to know when to hand-off a call to each other. The BSC coordinates these hand-offs, which is known as mobility management. Vocoding is where the digitized voice is converted to an appropriate format for the PSTN. When calls originate or terminate at a BTS from a different network (cellular or land-line), the BSC will request terrestrial circuits (land-line circuits) from the Mobile Switching Center (MSC) so that the call can be completed. Figure 2-10: Motorola GSM BSC, shows an example of what a BSC might look like.

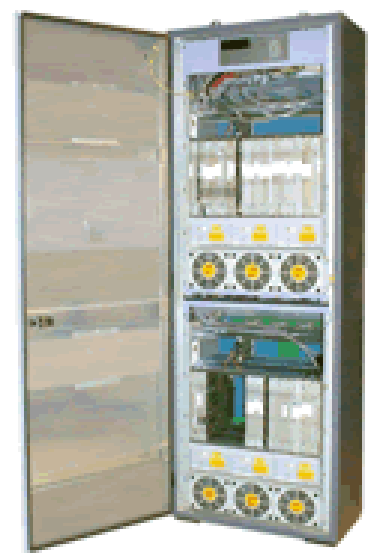

Figure 2-10: Motorola GSM BSC [4]

\subsubsection{Mobile Switching Center}

The MSC provides several vital functions for the cellular network. The MSC authorizes mobiles to access the network, coordinates roaming of mobiles, provides connections to the PSTN, traces billing and forwards 911 requests . MSCs are typically many racks or even rows of equipment, as shown in Figure 2-11: T-Mobile MSC. Depending on vendor and operator configuration, an average MSC could serve approximately 1 million subscribers, 6-8 BSCs and 500 BTSs.

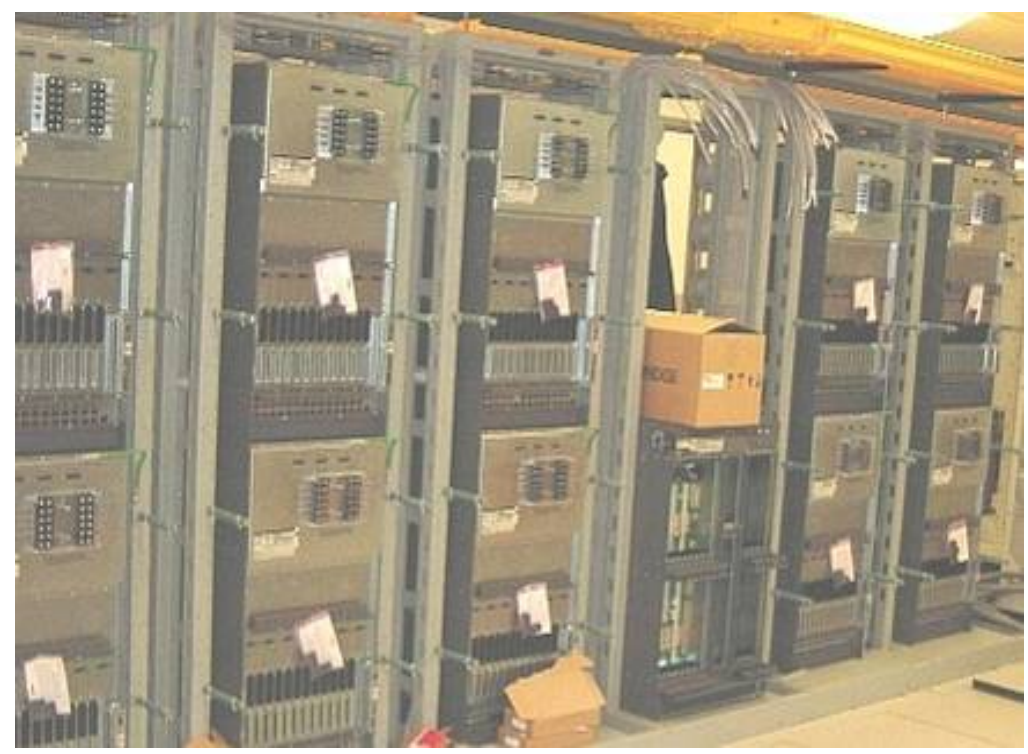

Figure 2-11: T-Mobile MSC [5] 
Within the logical MSC there are also two key functions that support network access and roaming: the Home Location Register (HLR) and Visitor Location Register (VLR). The HLR and VLR are the functions that allow operators to control which mobiles can roam between networks. The HLR contains the Mobile IDs of subscribers who are registered in the home network. If an MS roams into a visiting network, the visited MSC will register the roaming MS HLR information in its VLR. Without the registration data in the HLR the MS will be unable to access any network. The HLR and VLR are logical functions that do not have to be co-located with the MSC.

There is one exception to the HLR and VLR process; this is when the MS dials 911 for an emergency call. By FCC mandate, all cellular operators must connect 911 calls if the air interface between the mobile and the BTS is compatible. Upon the event of a 911 call, the MSC will automatically switch the call to the appropriate Public Safety Answering Point (PSAP) and also provide the location of the caller to the PSAP. Routing to the correct PSAP and providing the callers location to the PSAP operator is referred to as E911. This will be covered in detail in the emergency communications section of the report.

Usually, the MSC and BSC are kept in the same building called the Network Operators Center (NOC) or Mobile Telephone Switching Office (MTSO), one is shown Figure 2-12. Notice the multitude of microwave dishes on the tower. This is for the backhaul for the BTSs that are served by the BSC and MSC. Most emerging markets in India, China and Latin America will use microwave heavily due to lack of a fiber backbone for providing backhaul.

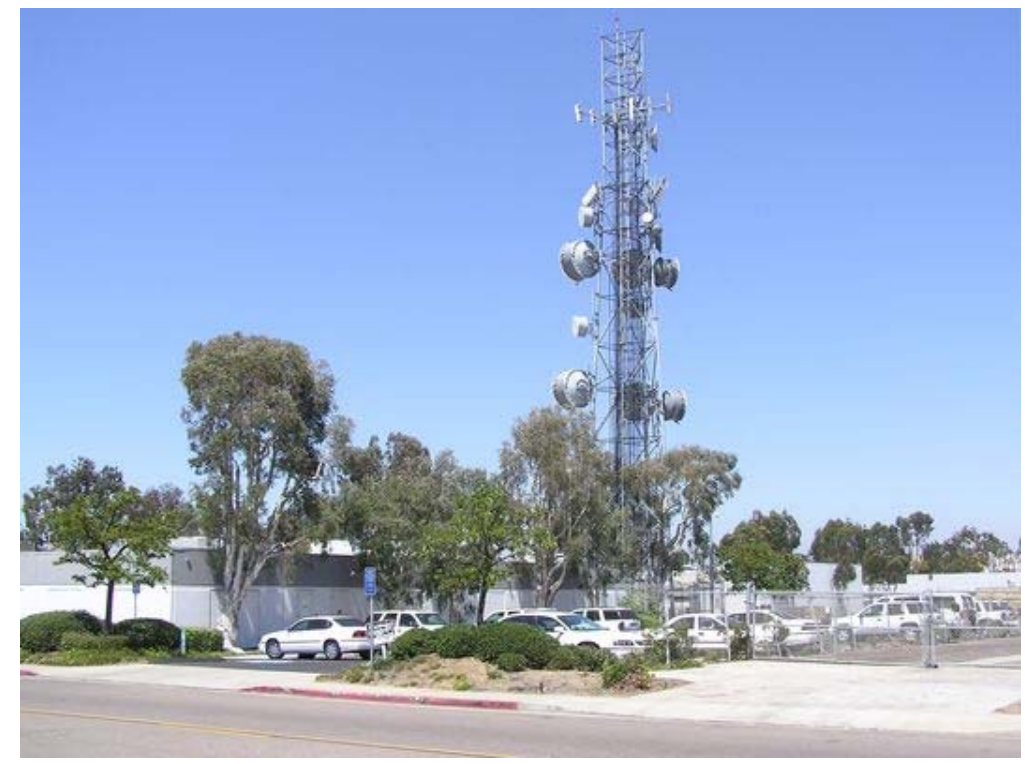

Figure 2-12: Verizon San Diego MTSO [6]

\subsubsection{Packet Data Node}

The Packet Data Node (PDN) is used for packet switched data or data switched directly to the internet. Its primary role is to maintain the link between the MS and the internet. The PDN does this using an important function called Mobile IP. Mobile IP is function which insures that the correct data gets to the correct MS. The PDN can provide other functionality such as authorization, mobility management and billing. In summary, the PDN can be seen as a gateway, router and tollbooth between the MS and internet.

\subsection{Cellular Technology: Generational Systems}

Cellular technologies are categorized by using the term "generation" or G. The International Telecommunications Union has specific criteria for each generation of technology; however, $G$ terms are widely used and loosely controlled. The following guidelines give a generic description of cellular generational systems:

\section{- $\quad 0 G$ and 1G - First Analog Systems}

- Brick phones and car phones 
- $\quad$ 2G - First Ubiquitous Systems

- Code Division Multiple Access (CDMA AKA IS-95)

- Global System for Mobile communications (GSM AKA IS-136)

- Integrated Digital Enhanced Network (iDEN)

- 3G - Wireless Data Faster than 1 Mbps

- CDMA 1x Evolution Data Only (EV-DO)

- Universal Mobile Telecommunications System (UMTS)

- High Speed Downlink Packet Access(HSDPA)

- High Speed Uplink Packet Access (HSUPA)

- 4G - Beyond 3G

- Potentially, Worldwide Interoperability for Microwave Access (WiMAX or 802.16)

- EV-DO-Rev C, (see Figure 2-18) predicted data speeds of up to $280 \mathrm{Mbps}$ [7]

- Long Term Evolution (LTE) with predicted data speeds of up to $278 \mathrm{Mbps}$. [8]

\subsubsection{Cellular Technology Families}

The most identifiable difference between cellular technologies is the techniques used on the air interface. The following tables provide key specifications for Code Division Multiple Access (CDMA) and Global System for Mobile communications (GSM). These two technologies form the two main technology families. Detailed discussion of each technology is not in the scope of this document; however, this information is given as reference for comparison.

Table 2-1, shows the CDMA family technologies. These technologies began with IS-95 A/B standards to later form CDMA 2000 or 1x technologies. IS-95A offered voice with some circuit switched data and offered packet data services in IS-95B. Unlike GSM, these early standards only defined the air interface and left network configurations up to the developing vendors. The $3 \mathrm{G}$ variants of CDMA, 1X, CDMA 1x Evolution Data Only (EV-DO), EV-DO Rev A, improved data rates and capacity by changes on the air-interface. EV-DO has no connection to the PSTN and offers only data services. Additionally, it uses a TDMA scheme for the BTS transmit. Most 3G CDMA devices will be dual mode providing voice using CDMA 1x or IS-95A/B and data using EV-DO. Verizon and Sprint the major operators offering EV-DO and EV-DO Rev A data service plans. 
Table 2-1: CDMA Technologies

\begin{tabular}{|c|c|c|c|c|c|}
\hline Technology Name & IS-95A & IS-95B & $1 x$ & $\begin{array}{c}\text { 1xEVDO Rev } \\
0 \\
\end{array}$ & $\begin{array}{c}\text { 1xEVDO Rev } \\
\text { A }\end{array}$ \\
\hline Generation & $2 \mathrm{G}$ & $2.5 \mathrm{G}$ & $2.5 \mathrm{G}$ & $3 G$ & $2.5 \mathrm{G}$ \\
\hline Multiple Access & CDMA & CDMA & CDMA & $\begin{array}{l}\text { CDMA } \\
\text { TDMA }\end{array}$ & $\begin{array}{l}\text { CDMA } \\
\text { TDMA }\end{array}$ \\
\hline Modulation & $\begin{array}{l}\text { BPSK } \\
\text { QPSK }\end{array}$ & $\begin{array}{l}\text { BPSK } \\
\text { QPSK }\end{array}$ & $\begin{array}{l}\text { BPSK } \\
\text { QPSK }\end{array}$ & $\begin{array}{l}\text { QPSK } \\
\text { QAM }\end{array}$ & $\begin{array}{l}\text { QPSK } \\
\text { QAM }\end{array}$ \\
\hline Year Published & 1993 & 1995 & 2002 & 2002 & 2004 \\
\hline $\begin{array}{c}\text { Voice Supported } \\
\text { Channel Bandwidth }\end{array}$ & $\frac{\text { Yes }}{1.25 \mathrm{MHz}}$ & $\frac{\text { Yes }}{1.25 \mathrm{MHz}}$ & $\frac{\text { Yes }}{125 \mathrm{MHz}}$ & $\frac{\text { VolP Only }}{125 \mathrm{MHz}}$ & $\frac{\text { VolP Only }}{125 \mathrm{MHz}}$ \\
\hline Circuit Switched Data & $14.4 \mathrm{kbps}$ & $64 \mathrm{kbps}$ & $64 \mathrm{kbps}$ & N/A & N/A \\
\hline Packet Switched Data & None & $64 \mathrm{kbps}$ & $144 \mathrm{kbps}$ & $\begin{array}{l}2.5 \text { Mbps Down } \\
154 \text { kbps Up }\end{array}$ & $\begin{array}{l}\text { 3.1 Mbps Down } \\
\text { 1.8 Mbps Up }\end{array}$ \\
\hline Comptability & IS-95A & IS-95A & IS-95A, IS-95B & $\begin{array}{l}\text { Dual Mode 1x } \\
\text { and EVDO } \\
\text { Mobiles }\end{array}$ & $\begin{array}{l}\text { Dual Mode 1x } \\
\text { and EVDO } \\
\text { Mobiles }\end{array}$ \\
\hline AKA & $\begin{array}{c}\text { TIA-EIA-95 } \\
\text { CDMA }\end{array}$ & TIA-EIA-95 & $\begin{array}{c}\text { 1x, 1xRTT, IS- } \\
2000, \\
\text { CDMA2000 1X, } \\
\text { 1X, cdma2000 }\end{array}$ & EV-DO & $\operatorname{Rev} A$ \\
\hline Development Group & Quallcomm & Quallcomm & 3GPP2 & 3GPP2 & 3GPP2 \\
\hline Notes & Air Interface & $\begin{array}{c}\text { Combined: } \\
\text { ANSI-J-STD- } \\
008, \text { IS-95. TSB } \\
74 \\
\end{array}$ & $\begin{array}{l}\text { Soft Handoffs } \\
\text { Power Control } \\
\text { More efficient }\end{array}$ & Al:IS-856 & Improved Al \\
\hline
\end{tabular}

Table 2-2, shows the GSM family technologies. 3GPP introduced its first standard known as GSM. Later General Packet Radio Service (GPRS) included connection to the internet or PDN, which as followed by Enhanced Data rates for GSM Evolution (EDGE) which offered improvements on the air interface to enhanced data rates. Universal Mobile Telecommunications System (UMTS) and High Speed Downlink Packet Access (HSPDA) technologies introduced major changes to the air interface to improved data rates. UMTS and HSPDA abandoned TDMA techniques and use a similar air interface to CDMA called Wideband CDMA (W-CDMA). Although W-CDMA is very similar in digital modulation techniques and channel coding to CDMA techniques listed above, it is not compatible with CDMA technologies. W-CDMA's major difference is its use of a $5 \mathrm{MHz}$ bandwidth instead of a $1.25 \mathrm{MHz}$ like regular CDMA. GSM networks and their variants are currently widely deployed by Cingular and T-Mobile in the US. 
Table 2-2: GSM Family Technologies [8][10][11]

\begin{tabular}{|c|c|c|c|c|c|}
\hline Technology Name & GSM & GPRS & EDGE & UMTS & HSPDA \\
\hline Generation & $2 G$ & $2.5 \mathrm{G}$ & $2.5 \mathrm{G}$ & $3 G$ & $3.5 \mathrm{G}$ \\
\hline Multiple Access & TDMA & TDMA & TDMA & W-CDMA & W-CDMA \\
\hline Modulation & GMSK & GMSK & 8-PSK & QPSK & $\begin{array}{c}\text { QPSK } \\
\text { 16-QAM }\end{array}$ \\
\hline Year Published & 1990 & 1998 & 2001 & 2005 & 2005 \\
\hline Voice Supported & Yes & Yes & Yes & Yes & Yes \\
\hline Channel Bandwidth & $200 \mathrm{kHz}$ & $200 \mathrm{kHz}$ & $200 \mathrm{kHz}$ & $5 \mathrm{MHz}$ & $5 \mathrm{MHz}$ \\
\hline Circuit Switched Data & $9.6-14.4 \mathrm{kbps}$ & $115 \mathrm{kbps}$ & $\mathrm{N} / \mathrm{A}$ & $\mathrm{N} / \mathrm{A}$ & $\mathrm{N} / \mathrm{A}$ \\
\hline Packet Switched Data & None & $30-80 \mathrm{kbps}$ & $160-384 \mathrm{kbps}$ & $1920 \mathrm{kbps}$ & $\begin{array}{c}14.4 \text { kbps Up } \\
\text { 2-3 Mbps Down }\end{array}$ \\
\hline Comptability & GSM & GSM & GSM/GPRS & $\begin{array}{c}\text { Dual Mode Mobiles } \\
\text { UMTS/GSM }\end{array}$ & $\begin{array}{c}\text { Dual Mode } \\
\text { Mobiles } \\
\text { HSDPA/GSM } \\
\end{array}$ \\
\hline AKA & GSM & GPRS & EGPRS & 3GSM, W-CDMA & HSDA \\
\hline Development Group & 3GPP & ETSI,3GPP & 3GPP & 3GPP & 3GPP \\
\hline Notes & $\begin{array}{c}\text { Europes First } \\
\text { Interoperable } \\
\text { Wireless }\end{array}$ & $\begin{array}{l}\text { Added Core } \\
\text { Network for } \\
\text { Data }\end{array}$ & Improved Al & $\begin{array}{c}\text { Combines W- } \\
\text { CDMA Al with GSM } \\
\text { Core }\end{array}$ & $\begin{array}{c}\text { Improved Al and } \\
\text { all IP Core with } \\
\text { IMS }\end{array}$ \\
\hline
\end{tabular}

\subsection{US Cellular Technology Composition and Operators}

As shown in, Figure 2-13: Subscribers by Technology, CDMA has a $\sim 49 \%$ proportional majority market share of subscribers in North America [12]-[14]. GSM captures $\sim 36 \%$ of the population with the remaining of the subscribers still using other technologies (iDEN and other analog cell phone technologies).

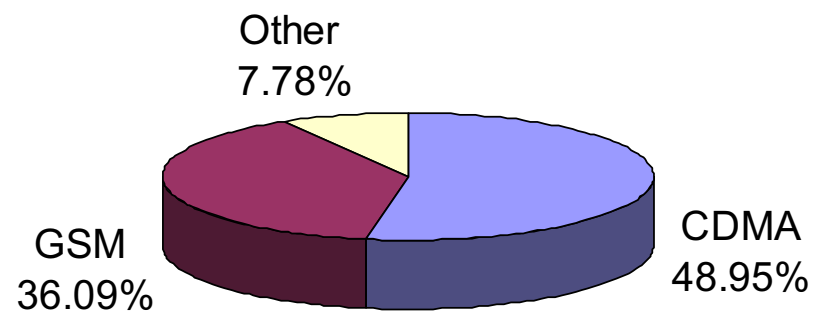

Figure 2-13: Subscribers by Technology [12]-[14]

The U.S. is made up of many different cellular operators; however the four major operators, Cingular (now AT\&T Mobility), Verizon, Sprint and T-Mobile make up a majority of the market share. Table 2-3: U.S. Major Cellular Operators, shows data for subscribers for each of the cellular operators and their technology type. 
Table 2-3: U.S. Major Cellular Operators as of October 2007 [15]-[23]

\begin{tabular}{|c|c|c|}
\hline Operator & Technology & $\begin{array}{l}\text { Subscribers } \\
\text { (millions) }\end{array}$ \\
\hline$\Leftrightarrow$ at\&t & $\begin{array}{l}\text { HSDPA, UMTS, EDGE, GPRS, } \\
\text { GSM, TDMA }\end{array}$ & 65.7 \\
\hline verizonwireless & $\begin{array}{l}\text { CDMA2000 1xEV-DO, CDMA2000 } \\
\text { 1x, CDMA }\end{array}$ & 63.7 \\
\hline $\begin{array}{l}\text { Sprint Nextel } \\
\text { Includes Sprint Network, Sprint } \\
\text { Nextel Network, and Boost Mobile Prepaid }\end{array}$ & $\begin{array}{l}\text { CDMA2000 1xEV-DO, CDMA2000 } \\
\text { 1x, CDMA (Sprint PCS), WiDEN, } \\
\text { iDEN (Nextel) }\end{array}$ & 54.0 \\
\hline T-Mobile T: . Mobile: & UMA, EDGE, GPRS, GSM & 26.9 \\
\hline clltel & $\begin{array}{l}\text { CDMA2000 1xEV-DO, CDMA2000 } \\
\text { 1x, CDMA, AMPS }\end{array}$ & 12.447 \\
\hline $\begin{array}{l}\text { TracFone } \\
\text { Includes NET10 }\end{array}$ & GSM, CDMA, TDMA & 8.803 \\
\hline ל US.Cellular & CDMA2000 1x, CDMA, TDMA & 6.010 \\
\hline Virgin Mobile & $\begin{array}{l}\text { CDMA2000 1xEV-DO, CDMA2000 } \\
\text { 1x, CDMA }\end{array}$ & 5.2 \\
\hline metroPCS & CDMA & 3.66 \\
\hline
\end{tabular}

Some operators do not own cellular infrastructure or licensed spectrum but have customers by using roaming agreements with other operators. These companies are known as Mobile Virtual Network Operators (MVNO). Tracfone and Virgin Mobile are both examples of these types of companies. In other words, these companies sell mobile phones and pre-paid minutes to their customers who will use another operator's network. Under roaming agreements with other operators these MVNOs essentially, resell minutes to their customers.

\subsubsection{Cellular Frequency Spectrum}

Frequency spectrum is the basis of the wireless communications business and can be one of the most costly capital investments to a wireless operator. To transmit on a cellular transmission frequency, cellular operators in the US must obtain a license from the Federal Communications Commission (FCC). The FCC will hold auctions for various regions or markets and cellular operators will then make bids for each market for a set of frequencies. The most recent license auction in 2006 raised a total of \$13.5 billion dollars [24]. Figure 2-14 and Figure 2-15 show examples of the frequencies and blocks used for cellular communications. 


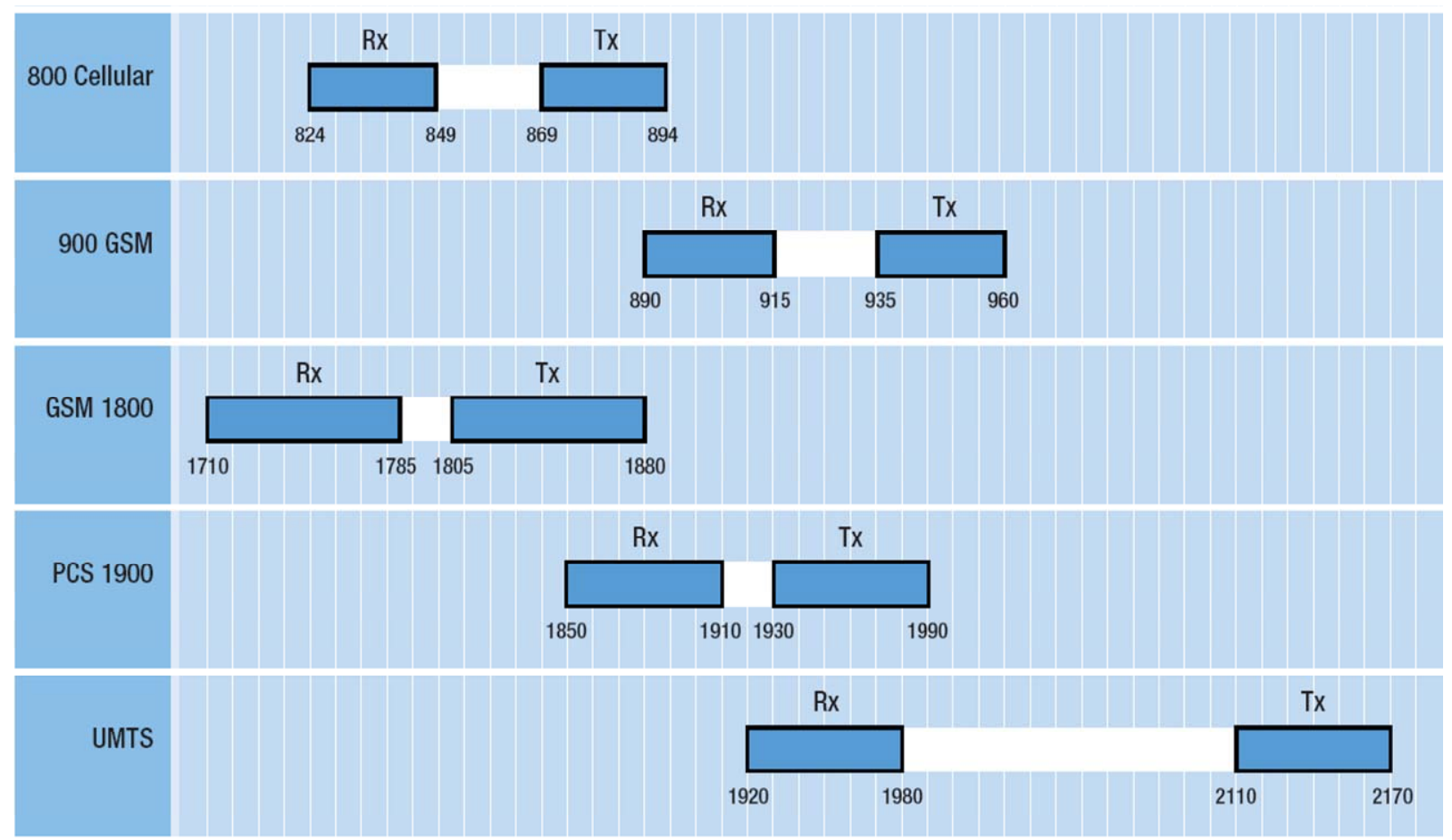

Figure 2-14: Cellular Frequencies in $\mathrm{MHz}$ [25]

\section{CELLULAR}

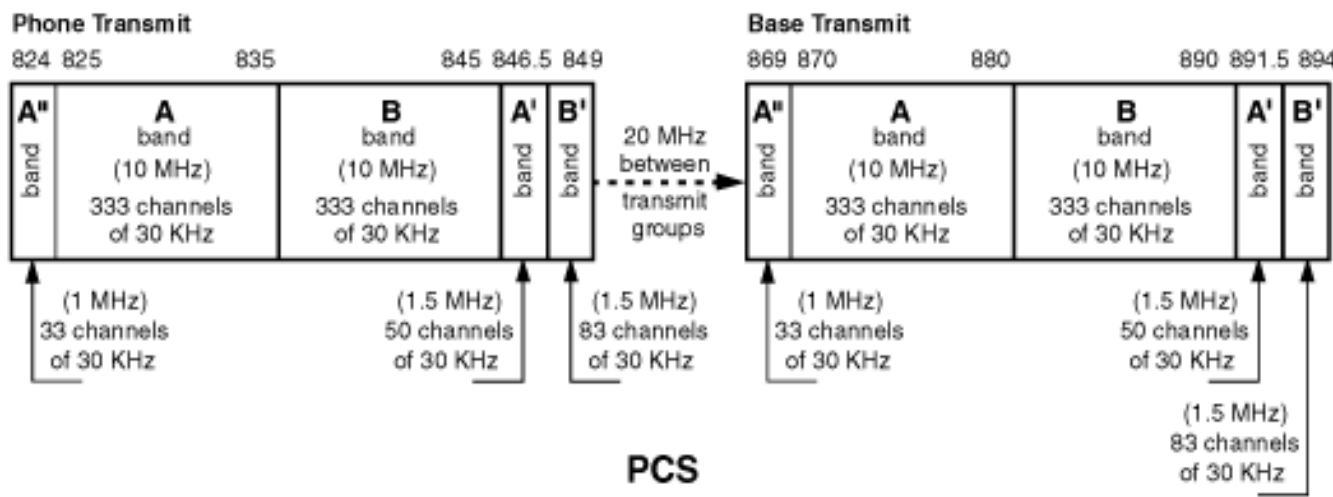

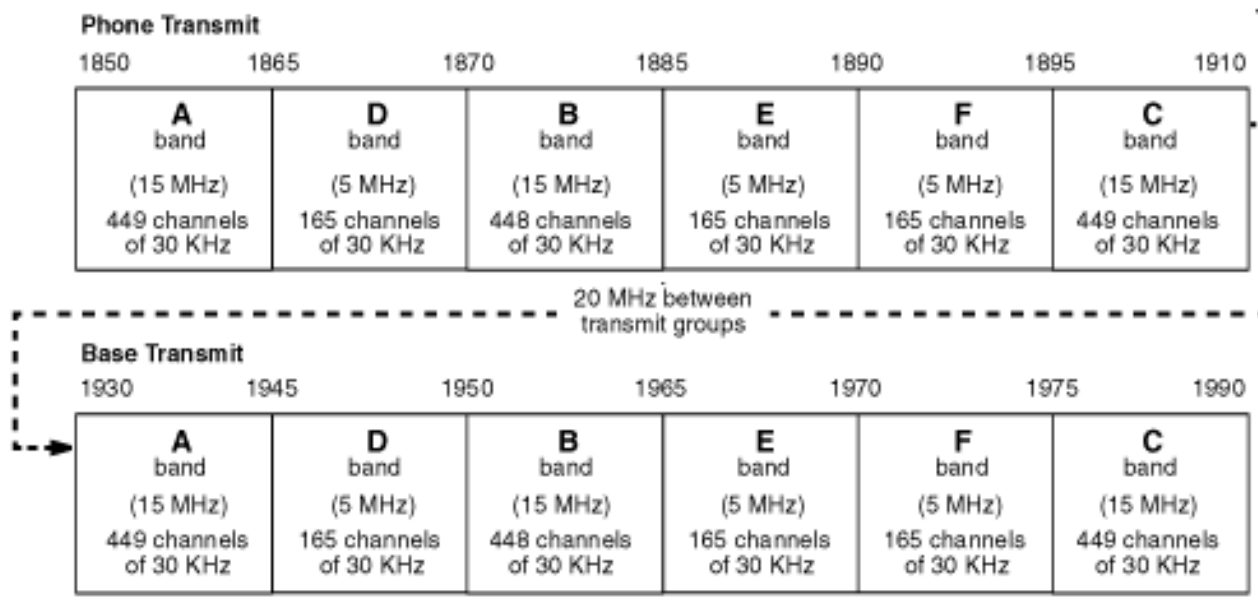

Figure 2-15: Cellular and PCS Frequency Blocks in MHz [26] 


\subsection{Disruptive Technologies: WiFi and WiMAX}

Wireless Fidelity (WiFi or 802.11) and WiMAX are what the cellular industry are calling "disruptive" technologies. Because these technologies provide faster data rates using unlicensed or cheaper spectrum and have the capability to disrupt the current cellular market's status quo by offering a competitive alternative. Another advantage is that these systems can tie directly into the Internet because the transport and network layers are similar (unlike cellular, which requires a PDN). WiFi and WiMAX networks take advantage of existing internet access practices and merely provide the physical link to the internet like an Internet Service Provider (ISP). These disruptive technologies have created new companies called Wireless Service providers (WISPs). These WISPs are now providing these new wireless high-speed data services.

Figure 2-16: Relative Wireless Data Speeds, gives a comparison of "peak" data speeds for each wireless technology. The chart shows in the same time it takes WiFi to download a music video of 5 megabytes, EVDO and HSPDA download approximately 0.5 mega-bytes. WiFi data download rates and ease of deployment have encouraged prolific WiFi deployments across the US and world. The US is estimated to have 42,843 free and paid WiFi hotspots. These hotspots can be found in cafes, hotels, libraries and also city wide municipal networks. Current estimates show approximately 400 municipal networks in operation or planned for construction in the US [27]. With the advent of Voice over IP (VoIP) services like Skype and Vonage, voice services of comparable quality to cellular could easily be provided on these systems.

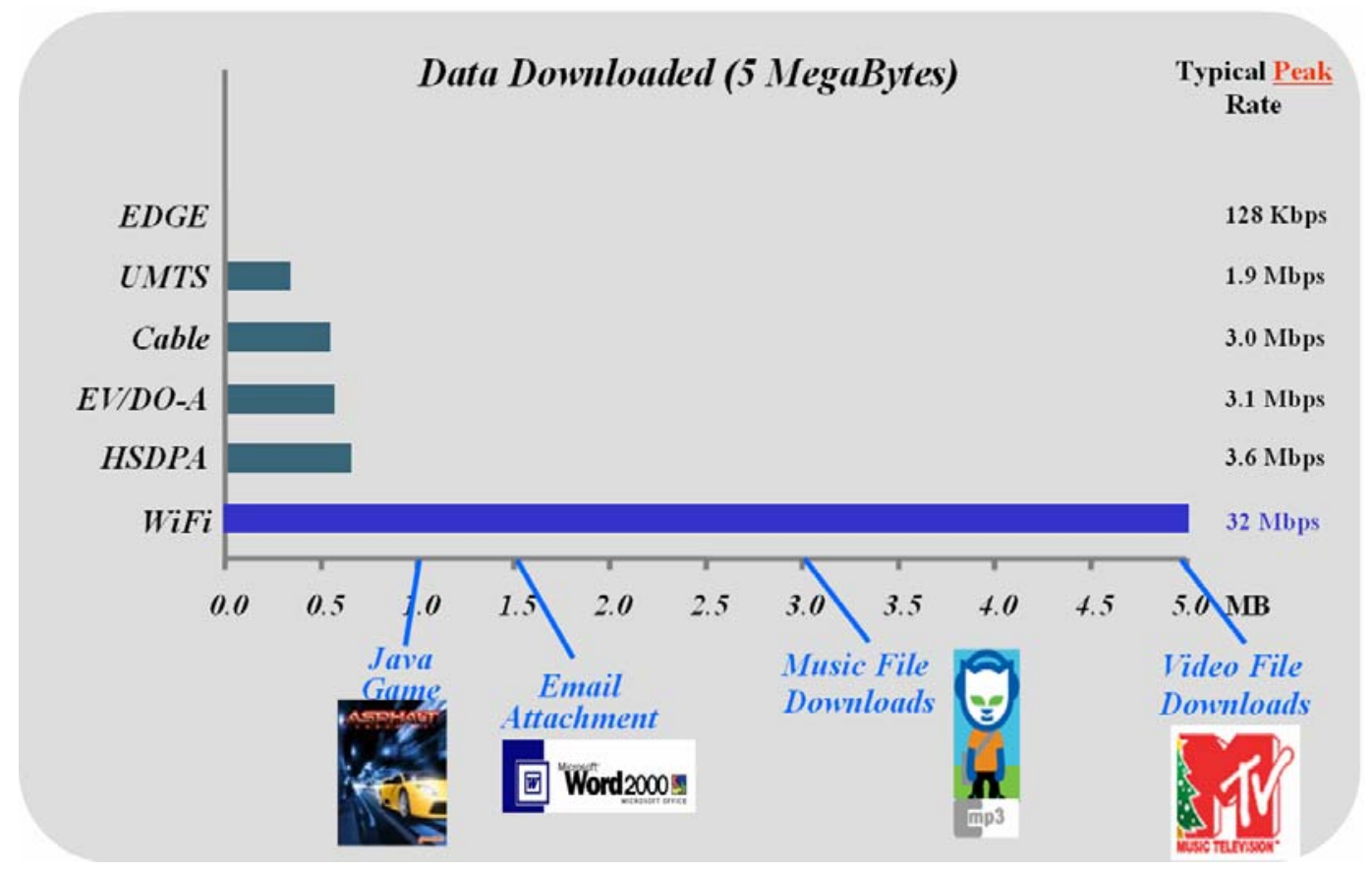

Figure 2-16: Relative Wireless Data Speeds

WiMAX was originally designed as a method for a wireless backhaul and last-mile deployments. WiFi in conjunction with WiMAX could add the future capacity and make hotspots easier to deploy. With WiMax backhauling WiFi hotspots it could be possible to provide the same ubiquity as cellular communications. See [28] 


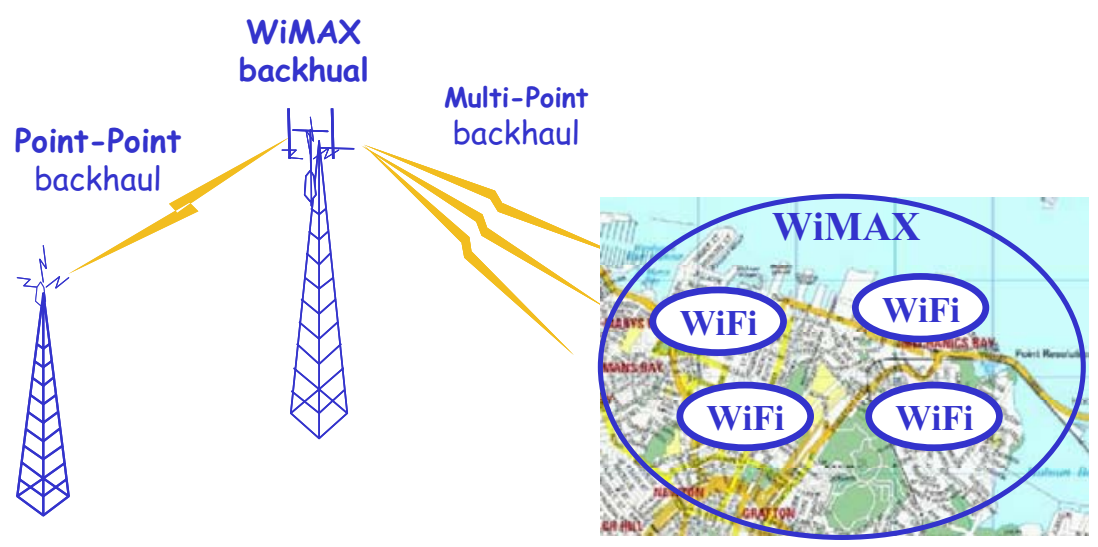

Figure 2-17: WiMAX Backhaul Supporting WiFi Hotspots [28]

Despite the advantages of WiFi, there are some associated problems such as significant security concerns, limited range and lack of mobility. On the other hand, WiMAX does have a longer range than WiFi and supports additional security and handover capability for seamless transitions. However, there are very few WiMAX networks and devices currently available. Additionally, both WiFi and WiMAX have less of a Doppler tolerance than cellular technologies. [29] Table 2-4 is provides a list of key specifications for reference for the WiFi and WiMAX technologies.

Table 2-4: 802.11 and 802.16 Capabilities

\begin{tabular}{|l|c|c|c|c|}
\hline \multirow{2}{*}{ Technology } & \multicolumn{3}{|c|}{ WiFi } & WiMAX \\
\cline { 2 - 5 } & $\mathbf{8 0 2 . 1 1 b}$ & $\mathbf{8 0 2 . 1 1 a}$ & $\mathbf{8 0 2 . 1 1 \mathrm { g }}$ & $\mathbf{8 0 2 . 1 6}$ \\
\hline Frequency band & $2.4 \mathrm{GHz}$ & $5.4 \mathrm{GHz}$ & $2.4 \mathrm{GHz}$ & $2.5 \mathrm{GHz}, 5.8 \mathrm{GHz}$ \\
\hline Max data rate & $11 \mathrm{Mbps}$ & $54 \mathrm{Mbps}$ & $54 \mathrm{Mbps}$ & $75 \mathrm{Mbps}$ \\
\hline Effective Data & $5 \mathrm{Mbps}$ & $32 \mathrm{Mbps}$ & $32 \mathrm{Mbps}$ & $32 \mathrm{Mbps}-74 \mathrm{Mbps}$ \\
\hline Bandwidth & $1 \mathrm{MHz}$ & $1.3 \mathrm{MHz}$ & $1 \mathrm{MHz}$ & $1-28 \mathrm{MHz}$ \\
\hline Modulation & QPSK & $\begin{array}{c}\text { QPSK } \\
\text { OFDM }\end{array}$ & $\begin{array}{c}\text { QPSK } \\
\text { OFDM }\end{array}$ & $\begin{array}{c}\text { QPSK } \\
\text { OFAM }\end{array}$ \\
\hline Range & $300 \mathrm{ft}$ & $225 \mathrm{ft}$ & $300 \mathrm{ft}$ & 4-6 Miles \\
\hline Availability & Worldwide & US & Worldwide & Worldwide \\
\hline
\end{tabular}

\subsection{Technology Trends}

\subsubsection{Changing Architectures and Devices}

The cellular world is a fast and changing, since the first cell phone we have seen rapid evolution in architectures and devices. Figure 2-18, shows some of these trends. In terms of trends in architecture, cellular architectures are migrating toward a packet core. The packet core is essentially an IP based media connection to the internet or other IP networks. This is very similar to the way WiFi (802.11) and WiMax (802.16) access points work. This new cellular core includes a new network element(s) referred to as IP Multi-media Subsystem (IMS) for GSM architectures or as Multi-media Messaging Subsystem (MMS) for CDMA architectures. These packet core architectures plan to offer a wider array of data services and capabilities for subscribers. This is especially important since cellular operators are depending on new data services to improve their revenue streams.

In terms of trends in devices, many new cellular devices are coming equipped with WiFi capabilities that allow them a wider variety of capabilities. These multi-mode mobiles are becoming more common and provide new opportunities for operators to add new service options. [30] Another new device trend is Home Gateways for end 
user. The gateways provide link to EV-DO or HSPA via a WiFi or Ethernet gateway for home computers. These gateways will allow users to seamlessly migrate from Cable or Digital Subscriber Line (DSL) high speed internet to EV-DO or HSPA for home use.

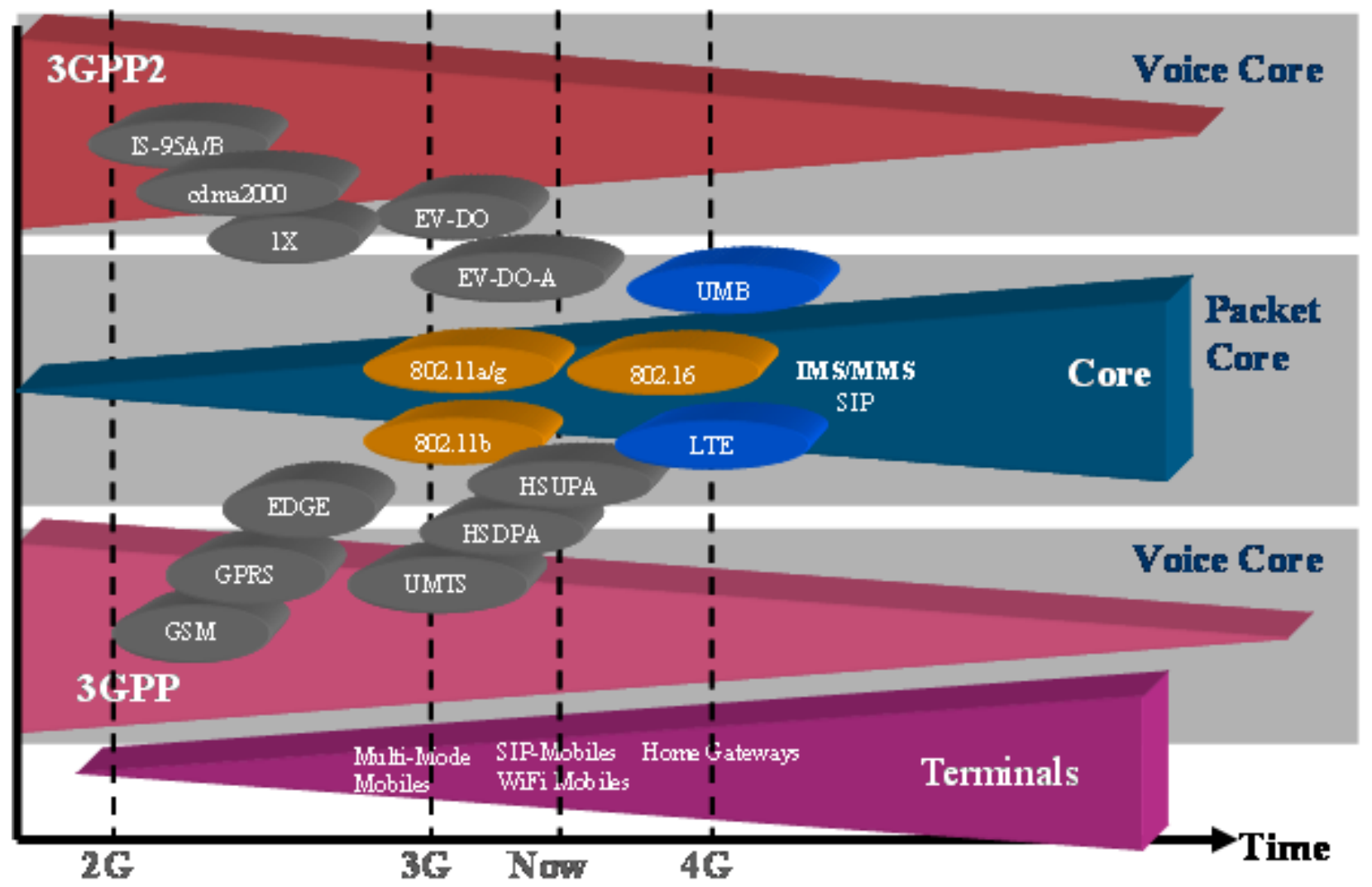

Figure 2-18: Cellular and Wireless Technology Trends

\subsubsection{Future Predictions}

Although technologies are changing fast, cellular operators in the US will limit their upgrades over the next several years. Currently in the U.S. cellular operators capital expenditures are outpacing their revenue growth. This will mean that operators will focus on using their existing capital investments (infrastructure and spectrum) to work more efficiently to make more money. [31] This could also mean reduction in operational expenses for operators (mergers and layoffs). In emerging markets like India, China and Latin America revenue growth is exceeding capital expenditures. This could mean that in the next 5-10 years these emerging markets could have more advanced wireless technologies than the US. [31] However, improving data revenues could create the capital necessary for U.S. operators to make upgrades sooner.

In addition to cellular operators exceeding their capital expenditures, WiFi networks will become much more prolific in coverage, especially because many new devices will come equipped with WiFi. As WiFi becomes more ubiquitous it could create tougher competition with cellular operators and could impact data revenues.

The much hyped WiMax, will take some market share. Clearwire Corporation is beginning to deploy WiMax network in Seattle and WiMax devices are being approved by the FCC. Clearwire also recently acquired WiMax spectrum from AT\&T to deploy its network. [32][33] Sprint-Nextel also has plans to deploy the technology with high hopes. [34] Both plan on offering end user WiMax devices. However, due to the competing cellular architectures, it is most likely that WiMax will take niche markets. [35] WiMax will break new ground but faces tough competition due to legacy architecture and devices. However, WiMax backhaul deployed with WiFi hotspots could acquire larger marker share due to the large number of WiFi devices already available. 
In terms of future predictions of technologies in the U.S. and the world, the US will have a combination of EVDO, CDMA 1X, GSM, UMTS and HSDPA infrastructure. All CDMA EVDO devices will have CDMA 1x capability and to date all UMTS/HSDPA devices have GSM capability [36]. GSM currently has $82 \%$ world wide market share and some operators have switched technology paths from CDMA to GSM. [13] Consequently, CDMA infrastructure upgrades and some devices will most likely be discontinued by operators outside of the US.

Conversely, existing GSM markets will migrate to UMTS/HSDPA technologies not EVDO. [37]

\subsection{Summary}

Wireless technologies come in many different forms. Digital modulation and multiple access techniques uniquely differentiate wireless technologies. Cellular systems although not exactly the same, have very similar architectures with network elements providing basic services to connect end users to the PSTN and the Internet. GSM and CDMA technology families form the two primary cellular technology families with GSM being the worldwide dominant technology. RF spectrum is limited resource and very valuable for cellular operators it forms the basis for their business. To increase efficiency of RF spectrum, wireless technologies are rapidly evolving to achieve faster bandwidths maximize use of available RF spectrum and to provide a wider array of data services. 


\section{REFERENCES}

[1] William C.Y. Lee, Wireless \& Cellular Telecommunications Third Edition, McGraw Hill 2005

[2] Louis Litwin and Michael Pugel, The principles of OFDM, www.rfdesign.com, January 2001

[3] Arnon Friedmann, Understanding OFDMA, the interface for wireless, Wireless NET Design Online, April 23, 2007, http://www.wirelessnetdesignline.com/showArticle.jhtml?printableArticle=true\&articleId= $\underline{199201230}$

[4] Motorola BSC, Motorola Inc. http://www.motorola.com/content.jsp?globalObjectId=2219-4452-4778

[5] Bergvik Telecom Installations, http://www.bergvik.com/telepref.asp

[6] Kramer.Firm's Wireless Site Gallery, Verizon MTSO San Diego

http://www.kramerfirm.com/cells/displayimage.php?album=search\&cat=0\&pos=19

[7] 3G - Ultra Mobile Broadband, CDMA Development Group, http://www.cdg.org/technology/3g_umb.asp

[8] Kapko, Matt, Verizon Wireless' LTE decision puts ball in UMB, WiMax court, RCR News, December 3, 2007

[9] Francis Botto, Encyclopedia of Wireless Telecommunications, McGraw-Hill TELECOM

[10] Michel Mouly and Marie-Bernadette Pautet,"The GSM System for Mobile Communications" Cell and Sys Publishers 1992

[11] Heikki Kaaranen, et al, UMTS Networks, Wiley 2005

[12] CDMA Development Group, 3Q 2006 Subscribers Statistics http://www.cdg.org/worldwide/cdma_world_subscriber.asp

[13] GSM Facts and Figures, GSM World, http://www.gsmworld.com/news/statistics/index.shtml

[14] CTIA Wireless, Current US Wireless Subscribers, http://www.ctia.org/

[15] AT\&T, 2007 Third-Quarter Results, October 23, 2007, http://www.att.com/gen/pressroom?pid $=4800 \&$ cdvn $=$ news \&newsarticleid $=24568$

[16] Verizon, 2007 Third-Quarter Results, October 29, 2007, http://investor.verizon.com/news/view.aspx?NewsID=863

[17] Sprint-Nextel 2007 Second-Quarter Results, , August 8, 2007 $\mathrm{http} / / /$ www2.sprint.com/mr/news_dtl.do?id=17680

[18] Deutsche Telekom, Interim Group Report, June 30, 2007, http://www.downloadtelekom.de/dt/StaticPage/43/39/94/DTAG_2007_H1_Report.pdf_433994.pdf

[19] Alltel, CONSOLIDATED HIGHLIGHTS AND OTHER FINANCIAL INFORMAT, September 30, 2007, http://media.corporate-ir.net/media files/irol/74/74159/3Q07 results/ $\underline{\text { 3Q07 Highlights.pdf }}$

[20] América Móvil, Third quarter of 2007 financial and operating report, September 2007, http://www.americamovil.com/docs/reportes/eng/2007_3.pdf 
[21] U.S. Cellular Reports, Second Quarter 2007 Results, August 7, 2007 http://phx.corporateir.net/phoenix.zhtml?c=106793\&p=irol-newsArticle\&ID=1037477\&highlight=

[22] Virgin Mobile USA IPO chalks up USD 412.5m, Telegeography CommsUpdate, October 11, 2007 http://www.telegeography.com/cu/article.php?article_id=20036

[23] Metro PCS, Third Quarter 2007 Subscriber Results, October 4, 2007, http://investor.metropcs.com/phoenix.zhtml?c=177745\&p=irol-newsArticle\&ID=1059282\&highlight=

[24] FCC Mulls Cos' Positions On Auction Of Prized Radio Spectrum, Corey Boles, Dow Jones Newswires, Cellular-News http://www.cellular-news.com/story/23987.php

[25] Application/Engineering Notes, Andrews Corporation, www.andrew.com

[26] Personal Communications Service, International Engineering Consortium, $\mathrm{http}: / / \mathrm{www}$. iec.org/online/tutorials/pcs/topic01.html

[27] Nicole Fabris, Municipal Wi-Fi Needs a Paradigm Shift, December 4, 2007 http://www.abiresearch.com/abiprdisplay.jsp?pressid=996

[28] Jeff Orr, 802.16 and 802.11: The right technology in the right place, WiMAX Forum http://www.WiMAXforum.org/news/downloads/righttechrightplace.ppt

[29] Mobile voice over $\mathrm{WiFi}$ at $130 \mathrm{~km} / \mathrm{h}$, Muniwireless, http://www.muniwireless.com/article/articleview/4605/1/5/

[30] Robert Jaques, Demand for WiFi mobiles to explode, vnunet.com 04 Jun 2007, http://www.vnunet.com/vnunet/news/2191336/demand-wi-mobiles-poised

[31] Yankee Group, New Opportunities for Vendors as Global Carrier Capex Accelerates, November 1, 2006 http://www.cellular-news.com/story/20155.php

[32] FCC Approves First WiMAX-Class Laptop Card to Be Offered by Clearwire, Cellular-News, May 2, 2007 http://www.cellular-news.com/story/23494.php

[33] Teresa Fausti, Helen Chungi, Clearwire Completes Acquisition of AT\&T Mobile WIMAX Spectrum, Clearwire Press Release, May 31, 2007, http://clearwire.com/company/news/05_31_07.php

[34] Roger Cheng, Sprint CFO: WiMax To Yield 10 Times Cost Performance, Dow Jones Newswires, May 30, $2007 \mathrm{http}: / / \mathrm{www} . c e l l u l a r-n e w s . c o m /$ story/24065.php

[35] HSPA to Dominate Mobile Broadband while WiMax goes Niche, Cellular News, Febuary 9, 2007, http://www.cellular-news.com/story/21894.php

[36] 3G Mobile Phones, GSM Arena, http://www.gsmarena.com/

[37] CDMA-Gone with the Wind?, ROA Group White Paper, August 2006 http://whitepapers.zdnet.com/whitepaper.aspx?\&docid=275001\&promo=Wireless 


\section{ACRONYMS}

\begin{tabular}{|c|c|}
\hline AT & Access Terminal \\
\hline $\mathrm{BCS}$ & Base Station Controller \\
\hline BPSK & Binary Phase Shift Keying \\
\hline BSC & Base Station Controllers \\
\hline BTS & Base Transceiver Station \\
\hline CDMA & Code Division Multiple Access \\
\hline DSL & Digital Subscriber Line \\
\hline EDGE & Enhanced Data rates for GSM Evolution \\
\hline EVDO & 1x Evolution Data Only \\
\hline FDMA & Frequency Division Multiple Access \\
\hline FSK & Frequency Shift Keying \\
\hline GMSK & Gaussian Minimum Shift Keying \\
\hline GSM & for Mobile communications \\
\hline GPRS & General Packet Radio Service \\
\hline HLR & Home Location Register \\
\hline HSPDA & High Speed Downlink Packet Access \\
\hline HSUPA & High Speed Uplink Packet Access \\
\hline IMS & IP Multi-media Subsystem \\
\hline MA & Multiple Access \\
\hline MMS & Multi-media Messaging Subsystem \\
\hline MSC & Base Station Control \\
\hline MSK & Minimum Shift Keying \\
\hline MTSO & Mobile Telephone Switching Office \\
\hline MVNO & Mobile Virtual Network Operator \\
\hline NOC & Network Operators Center \\
\hline PDN & Packet Data Node \\
\hline PSAP & Public Safety Answering Point \\
\hline PSK & Phase Shift Keying \\
\hline PSTN & Public Switched Telephone Network \\
\hline QPSK & Quadrature Phase Shift Keying \\
\hline RF & Radio Frequency \\
\hline SIP & Session Initiated Protocol \\
\hline TDMA & Time Division Multiple Access \\
\hline UMB & Ultra Mobile Broadband \\
\hline UMTS & Universal Mobile Telecommunications System \\
\hline
\end{tabular}


VLR Visitor Location Register

VoIP Voice over IP

W-CDMA Wideband CDMA

WiFi Wireless Fidelity (AKA 802.11)

WiMAX Worldwide Interoperability for Microwave Access

WISP Wireless Internet Service Provider 


\section{Emergency Communications}

Emergency communications are the methods of communication in which first responders use to respond to incidents. There are several components to this, from basic 911 to first responder communications. First responders use a variety of wireless networks, called Land Mobile Radio (LMR) networks, to communicate with each other. The following section explains the basics public safety networks function, E911, the different standards of LMR, and problems and potential solutions to the interoperability problem.

\subsection{E911}

Enhanced 911, or E911, is the method by which first responders know how to respond to an incident. The primary function of E911 is to identify the location of the caller once the call has been made. There are two primary forms of E911: Wireline E911 and Wireless E911.

\subsubsection{Wireline E911}

Figure 3-1 gives a pictorial representation on how 911 works in a wireline network:

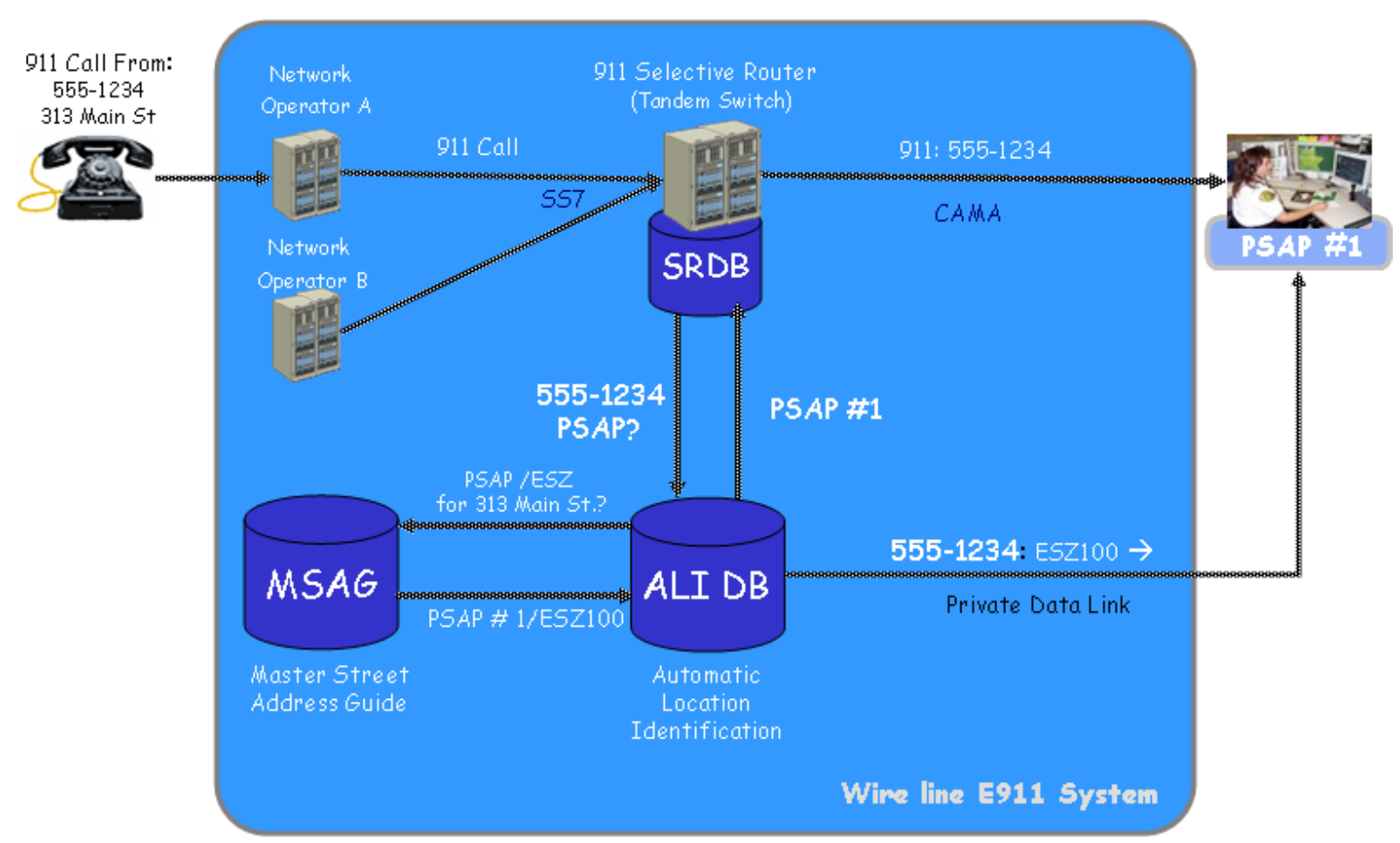

Figure 3-1: Wireline E911

In Figure 3-1, phone number 555-1234 makes a 911 phone call. The call goes through the operator's network and it is forwarded to a special switch called a 911 selective router. This selective router may have multiple operators connected to it. The purpose of the selective router is to route the 911 call to the public safety answering point (PSAP) serving the geographic area of the call. To do this, the selective router uses information from a local database called the selective router database (SRDB), or it gets the information from another 911 database called an Automatic Location Identification Database (ALI DB), which identifies PSAP from the customers service address. [38]

In addition to correct 911 routing, the PSAP also requires information of which service (fire, police, EMS) and which jurisdiction (city, county, and state) dispatch to notify. Emergency Service Zones (ESZ) define specific geographic areas and their associated services. These ESZs stored in the Master Street Address Guide (MSAG). In order to get this information to the PSAP, the ALI DB can query the MSAG, which sends the ESZ of the call to the PSAP. After the PSAP receives all this information, it can notify the appropriate dispatch agency, as shown in Figure 3-2: PSAP Calling Dispatch. [39] 


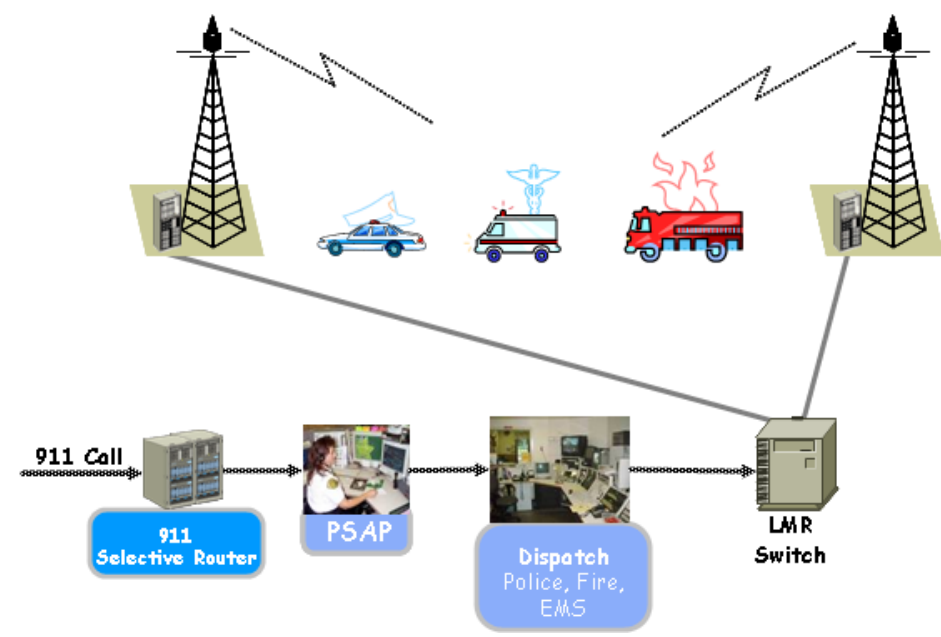

Figure 3-2: PSAP Calling Dispatch

Once the dispatch agency receives information that there is an emergency, it will radio its responders to notify them of the incident. This is usually done through a LMR wireless network. LMR networks will be discussed in more detail in the latter parts of this section.

\subsubsection{Wireless E911}

Wireless E911 is very similar to the E911 for wire line. The primary differences are in the way the mobile phone is dynamically located.

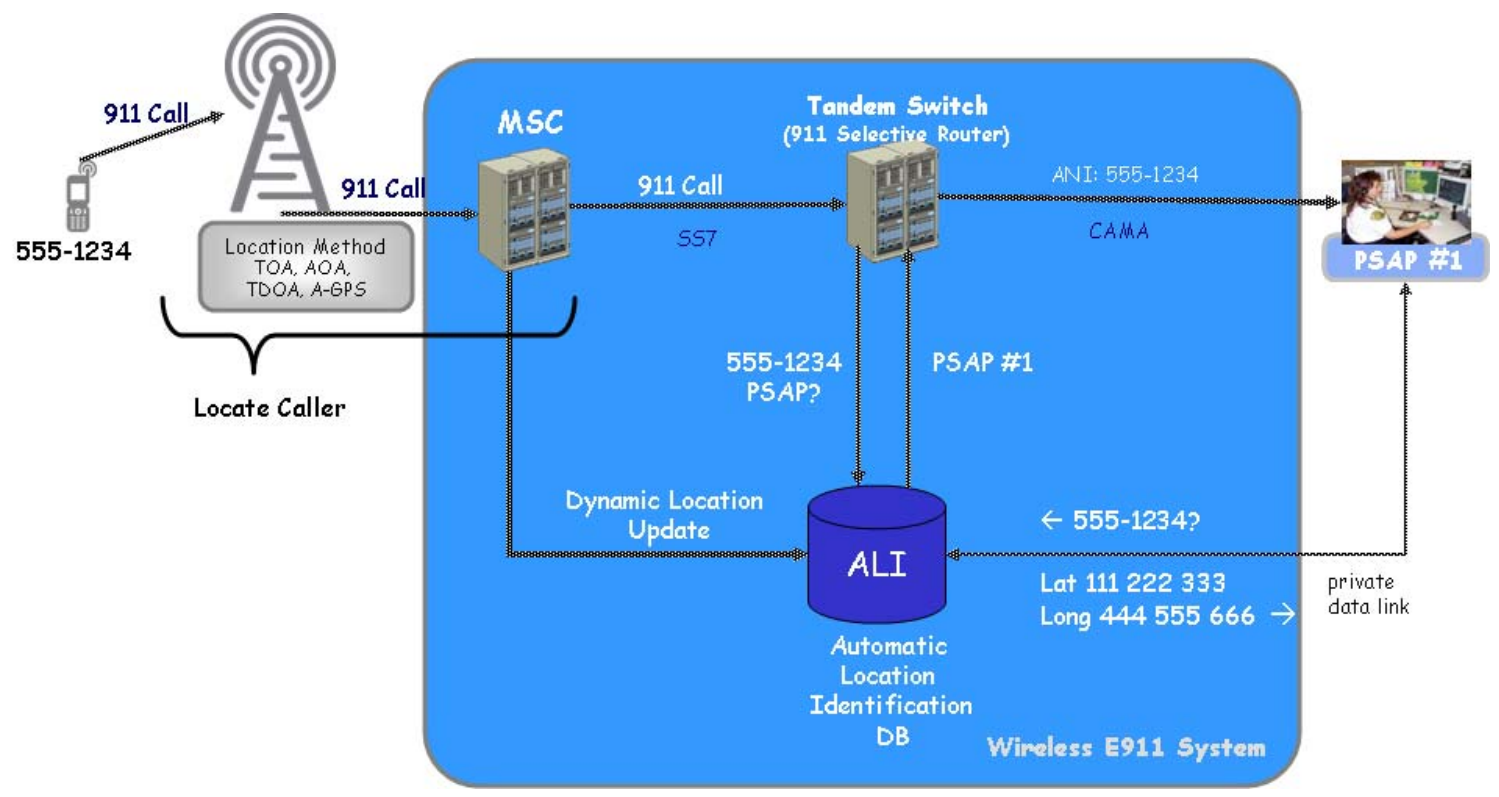

Figure 3-3: Wireless E911 [40]

Wireless E911 uses a few different methods to determine the location of the caller these methods are depicted in Figure 3-4: Methods for Finding Caller Location. 


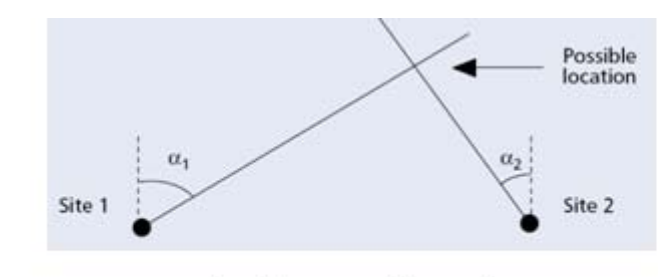

Angle of Arrival (AOA)

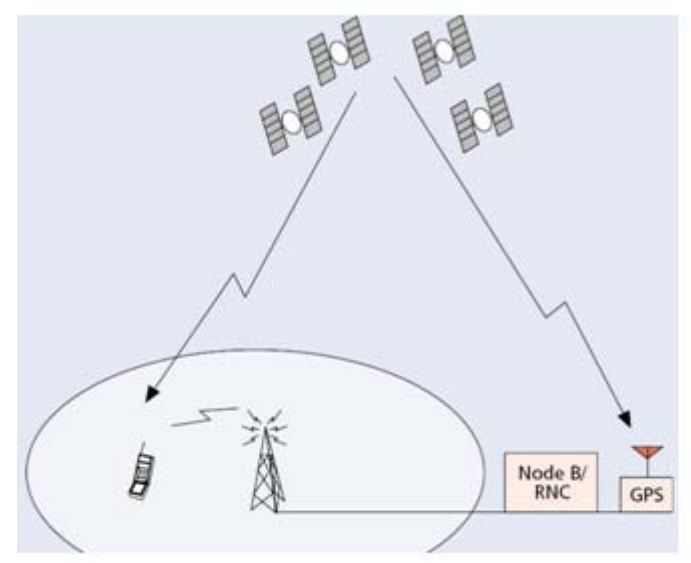

Assisted GPS (A-GPS)

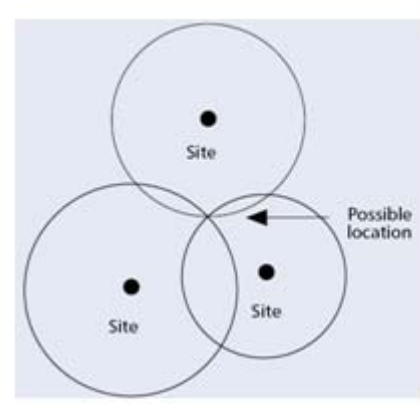

Time of Arrival (TOA)

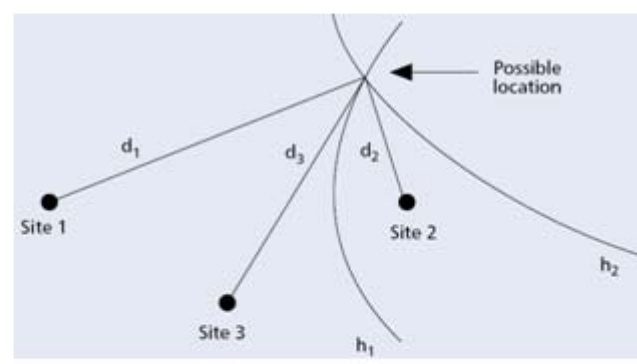

Time Difference of Arrival (TDOA)

Figure 3-4: Methods for Finding Caller Location [41]

While the specifics of each technique will not be discussed in detail, it's important to note the following: CDMA carriers in the U.S., use the A-GPS method to determine the mobiles location, while GSM operators decided to use one of the other methods. In comparison, A-GPS technique requires GPS chip in the handset and a single BTS, while all other techniques require two or three BTS for an accurate mobile device location. In 2001, the FCC ordered Sprint and Verizon to have A-GPS capabilities on $100 \%$ of new digital handsets activated nationwide. [42] FCC gave similar orders to GSM operators utilizing the network solutions to "triangulate" the mobiles location. In any case, when a 911 call is made the location is updated in the ALI DB, then the same method for wireline E911 is used to determine the PSAP and the location of the caller using GPS coordinates.

In summary, both the wireless and wired networks require terrestrial assets to be functional to process, receive and route 911 calls to the proper PSAP. Both depend on switching centers, 911 routers, backhaul that runs between and the 911 call centers. If any one of these single points fails, then 911 networks will not fully function.

\subsection{Next Generation 911}

Next Generation 911 (NG 9-1-1) is the next evolution of 911 systems after E911. The concept behind NG 9-1-1 is to develop and deploy an organization of systems that are IP-based, low cost, and can support multiple applications. Similar to modern communications systems, this idea allows PSAP operators to choose from multiple streams of information, whether live video or Mobile Messaging Service (MMS), and intelligently distribute information to first responders.

Although the standards are still in development, the Department of Transportation has published both the NG 9-1-1 System Description and High-Level Requirements Document and the NG 9-1-1 Concept of Operations. [43][44] Additionally, NENA has published what it considers the blueprint of NG 9-1-1, as shown in Figure 3-5. [45] 


\section{The Future of 9-1-1 and Emergency Communications}

\section{A Blueprint for a 'System of Systems'}

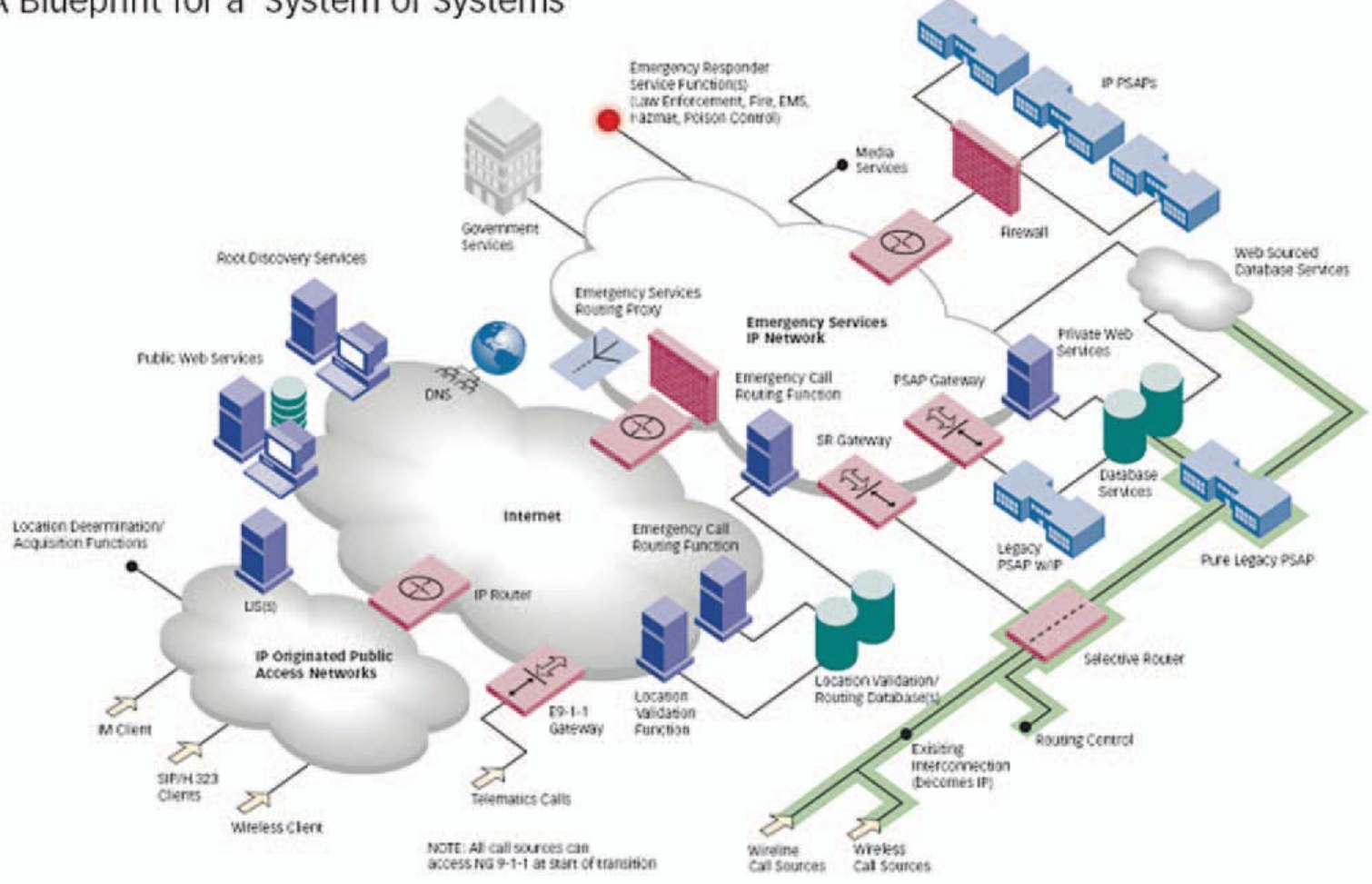

Figure 3-5: NG911 Blue Print [42]

\subsubsection{Network Architecture}

The NG 9-1-1 Concept of Operations calls for an overhaul of the PSAP architecture. The major changes of the architecture include IP based systems, geographically tiered PSAPs, and multimedia capabilities throughout multiple agencies and PSAPs. These changes were determined to be a necessity for emergency systems (i.e., PSAP), first responder communications, and response networks to be able to cope with the ongoing technology evolution.

By converting the network to an IP-based architecture, the emergency network is able to receive data from any source and distribute it to multiple entities intelligently. In current systems, very rarely are the multitudes of databases tied together and able to be accessed by anyone requiring information. For example, a simple task of running a license plate number during a routine traffic stop often requires a call to dispatch. Dispatch then queries a license plate database and then pass the information by voice to the officer. By using established Internet Engineering Task Force (IETF) standards, this data can be easily accessed by anyone. Using databases that connect to servers and relay the information needed can save not only time, but lives as well.

Geographically tiered PSAPs are not a new concept, but one that is coming to fruition in the NG 9-1-1 Concept of Operations. The principle behind this concept is that if a PSAP is overloaded or out of service, a backup PSAP will have the calls routed to it. This is done in two ways. The first way once a PSAP reached its call taking capacity, the manager can manually route calls to a neighboring PSAPs. This relieves the overloaded PSAP while still providing services to the caller. The second way that this is accomplished is in the event that the PSAP is taken offline, through either natural disaster or other catastrophic event, the NG 9-1-1 architecture will route the calls to a geographically separated PSAP. This concept is shown in Figure 3-6. 


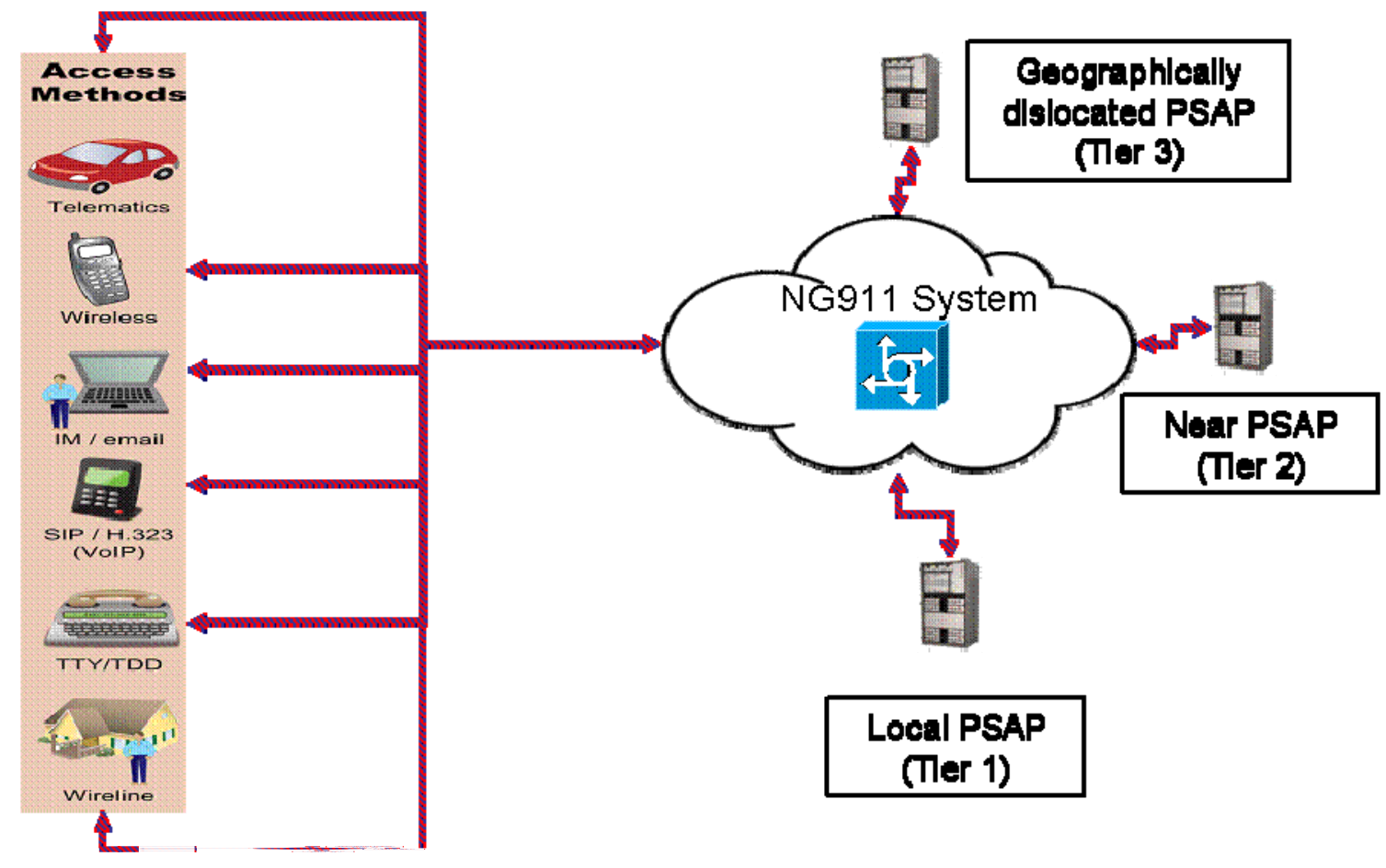

Figure 3-6: Tiered NG 9-1-1 Architecture

The ability to receive data from a multitude of media types is increasingly important to the first responders and decision makers. The NG 9-1-1 Concept of Operations requires the system to accept streaming video, text and other data in addition to the current voice and teletypewriter and telecommunications device for the deaf capabilities. The ability to stream video from a police car to the PSAP or incident commander is a valuable tool that will enhance response capabilities. Additionally, the ability for EMS crews to send live patient data such as vital signs to hospitals during transit will allow medical teams to better treat critical patients.

\subsubsection{Location Based Services}

The E911 standard introduced mobile location based services. The NG 9-1-1 refines the requirements for these services and goes so far as to include IP-based phone services. The NG 9-1-1 requirements state that not only will accurate location information be displayed to the PSAP, but fallback information will also be available. In other words, if a cellular device initiates a 911 call, the GPS coordinates (or other highly accurate method) will be sent to the PSAP. Should the immediate location not be available, the PSAP will have the option of retrieving fallback location information. This information may be as simple as identifying which cell the caller is located.

According to the System Initiative System Description, any device connected to the internet will have access to 911 services. Currently, this may not be the case with some Voice over IP (VoIP) phone services such as Skype. In the case of VoIP services, a user would have to call one of the PSAP's dedicated administrative lines in order to receive 911 services. Additionally, other wireless IP devices (WiMax and WiFi) will need to communicate location information through the NG 9-1-1 network

\subsubsection{NG 9-1-1 Impacts to the ACN}

The key to planning the ACN is to ensure that it is capable of adapting to future technologies. In the emergency communications arena, this means being able to adapt with the evolutions of NG 9-1-1. The recommended architecture for the ACN calls for an IP core as the heart of the system; this lends itself to the architecture of the NG 9-1-1 system. In addition, the ACN will have to support the ability to send and receive various types of voice, data and video over the dedicated first responder radio links. This means planning a system that has enough bandwidth to handle the potential flow of information between the command center and the first responders, as well as having equipment that can handle the varying types of data. 


\subsection{Public Safety Wireless Networks}

\subsubsection{Land Mobile Radio}

Most, if not all, public safety wireless communications networks are Land Mobile Radio (LMR) networks. Figure 3-7 illustrates the basic components of the dispatching function with an LMR network.

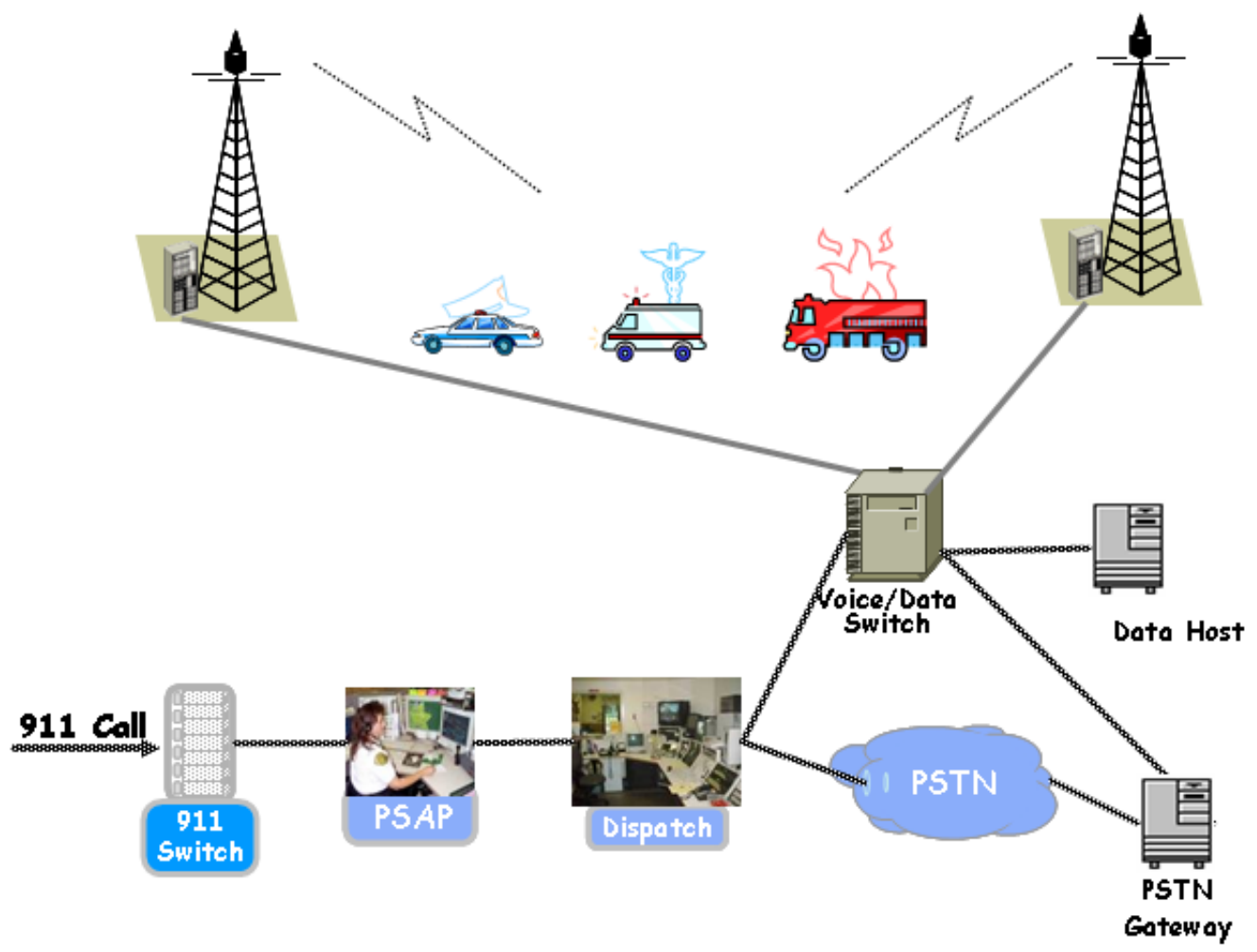

Figure 3-7: First Responder Network

The best way to envision a public safety network working is to examine the event of an emergency from a 911 call. When a 911 call is dialed by a wireless or wireline user, the switch automatically forwards the call and caller information to the associated PSAP. The PSAP receives the emergency information and then contacts dispatch to call the appropriate services to the emergency. Dispatch will have a connection to the LMR network and contacts the public safety required to respond to the emergency.

LMR network architectures are similar and less complex than cellular architectures. LMR radio systems can be as simple as a single repeater system or a trunked system covering a wide geographic area. Many of these architectures and LMR air interfaces are not on standard-based LMR systems. This is where the majority of U.S. public safety community is today. However, there are two main competing standards today: P25 and TErrestrial Trunked RAdio (TETRA).

\subsubsection{P25}

In the early 1990s, Association of Public-Safety Communications Officials (APCO) formed radio Project 25 (P25) to develop a standards-based digital LMR system. These P25 standards were used to build common interfaces between network elements so that LMR equipment vendors could be interoperable. P25 objects were help public safety officials achieve interoperability between different networks and put public safety LMR onto a technology upgrade path. To date, this LMR communications system is probably the most well known and adopted LMR standard in the U.S. operating in 134-174 MHz, 406-512 MHz, and 764-859 MHz frequencies. [39] However, many first responders have not deployed P25 systems because upgrading existing LMR networks is expensive. Although, not completely deployed across the U.S., it does have some advantages since there are multiple vendors for infrastructure and handsets. Currently there are 600 P25 networks deployed across the world. [46] 


\subsubsection{TETRA}

TErrestrial Trunked RAdio (TETRA), an ETSI standard, was first version published 1995, is a European based digital LMR system. It was specifically designed for use by government agencies and specifically emergency services, and the military. TETRA was also developed as a standards based technology for interoperable communications networks. Although not as popular in the U.S., TETRA currently has over 1000 networks deployed worldwide. [46] There are fundamental differences in the technologies although both provide essentially the same features.

\subsection{Interoperability Problems and Solutions}

Public safety networks in the United States are mostly disparate. Each level of government and each functional area in government could have different communications network. This has lead to the long debated problem of how to get interoperable communications between public safety providers. According to the National Task Force on Interoperability[39] [47]:

"There are approximately 2.5 million public safety first responders in the United States working for 18,000 State and local law enforcement agencies, 26,000 fire departments and over 6,000 rescue departments, plus Federal and tribal law enforcement, and other agencies such as Federal and State emergency management, transportation, and the public utilities who need to talk to one another during critical incidents."

Additionally, according to report from the U.S. House of Representatives by Tom Davis [48]:

"Over 90 percent of the nation's public safety wireless infrastructure is financed, owned, operated, and maintained by the more than 60,000 individual local jurisdictions, police, fire and emergency medical services that serve the public."

In addition to the different jurisdictions, there are also varieties of different vendors that make equipment based on proprietary standards. In other words, even if different radios operate at the same frequency it is possible they still could not be interoperable since the air interface could use different protocols.

\subsubsection{Network Patch Interconnect Solutions}

Although there is interoperable public safety and military networks, interconnect solutions have be utilized to bridge these gaps. Figure 3-8 shows an interconnect solution between public safety networks:

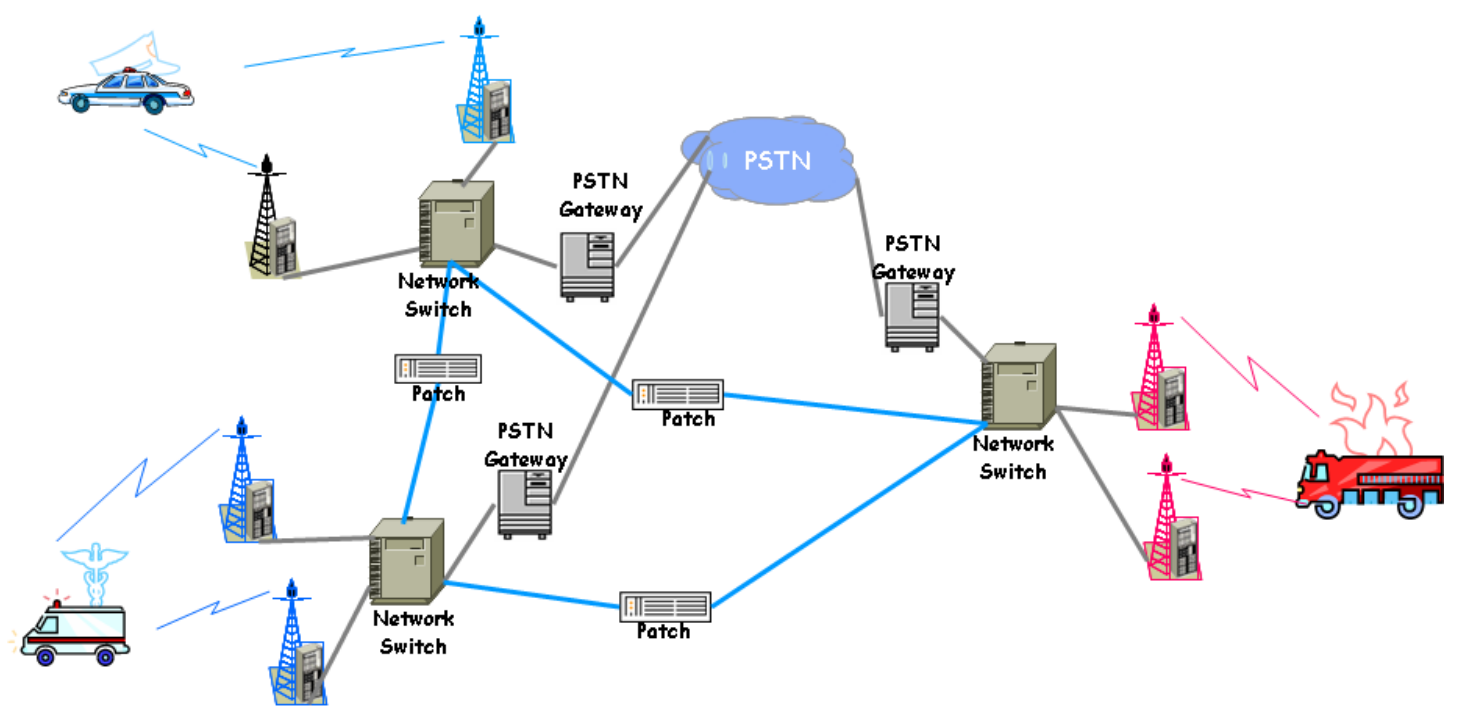

Figure 3-8: LMR Interconnect Solution 
Typically, what is done is a patch is put between each of the networks to enable communications between public safety workers. [39]

\subsubsection{IP Core Network Interconnects}

For interoperability between networks, IP cores are being utilized with proprietary protocols to create a seam between different technologies. These solutions work by forming the networks around an IP core. Additionally these solutions tie together LMR radios, cellular and WiFi to form together a single cohesive network. Networks of this type have the following generic architecture shown in Figure 3-9.

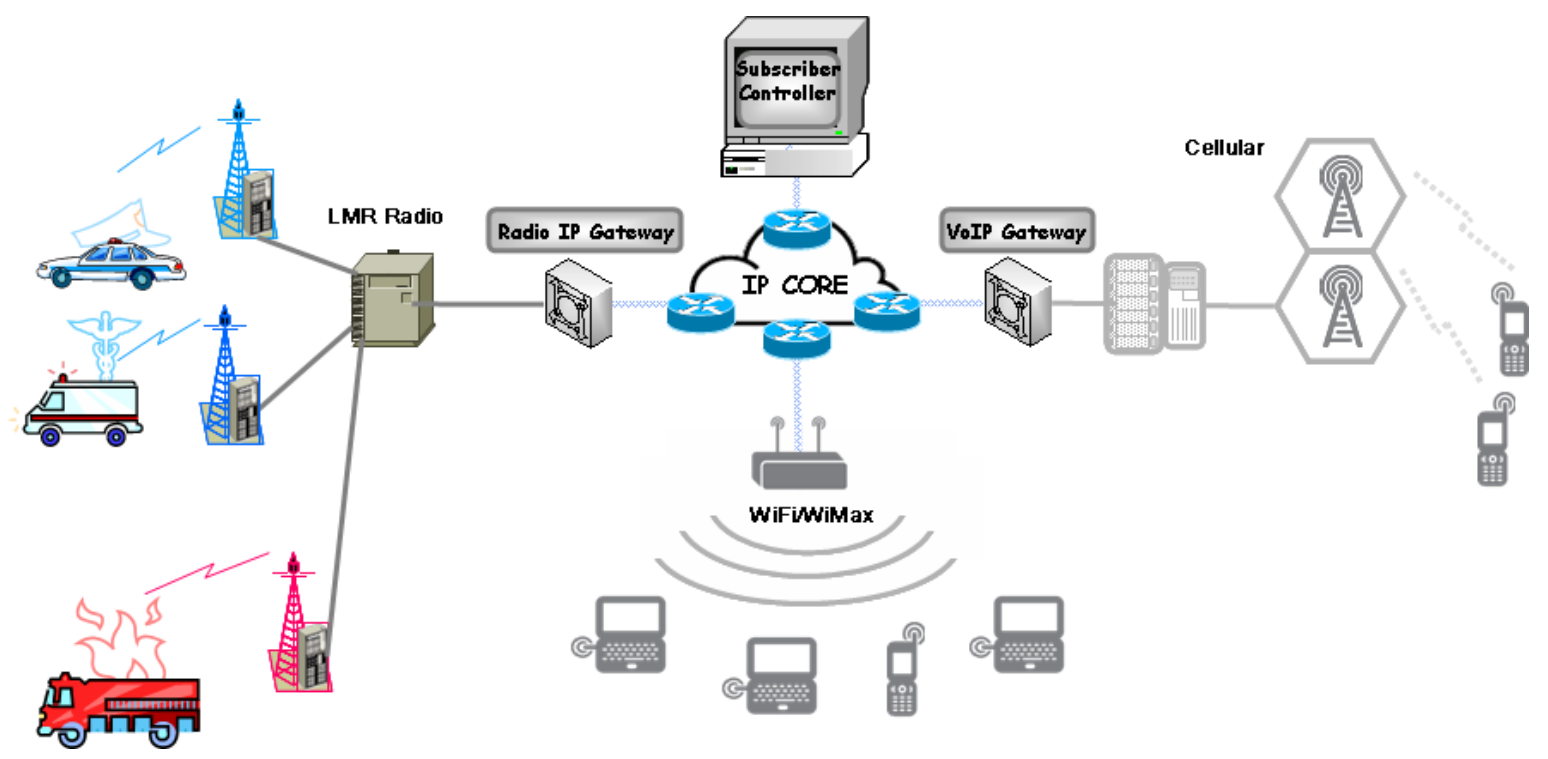

Figure 3-9: IP Core Interconnect

Solutions of this nature are also being deployed in military and public safety systems. RIPERNET, MobileNet and COCO are examples of technologies that use architecture of this nature. [49][50][51] In general, this architecture is very powerful since it allows for a single interconnect rather than a mesh as in the previous interconnect solution. The IP core allows for varieties of different technologies to intercommunicate using a solution other than dedicated circuits. Additionally, this architecture allows for a wider variety of capabilities and applications within the network.

\subsubsection{MHz Public Safety Network}

The $700 \mathrm{MHz}$ Band spectrum, which runs from 698-806 MHz, is currently occupied by television broadcasters. As a result, of Digital Television and Public Safety Act of 2005 (DTV Act), these $700 \mathrm{MHz}$ frequencies will either be auctioned for other wireless commercial services and or made available for public safety. The DTV Act set a firm deadline of February 17, 2009 for the completion of the DTV transition with the auction of $700 \mathrm{MHz}$ Band licenses scheduled for January 16, 2008. Figure 3-10, shows the planned spectrum usages of the $700 \mathrm{MHz}$ Band. [52] 


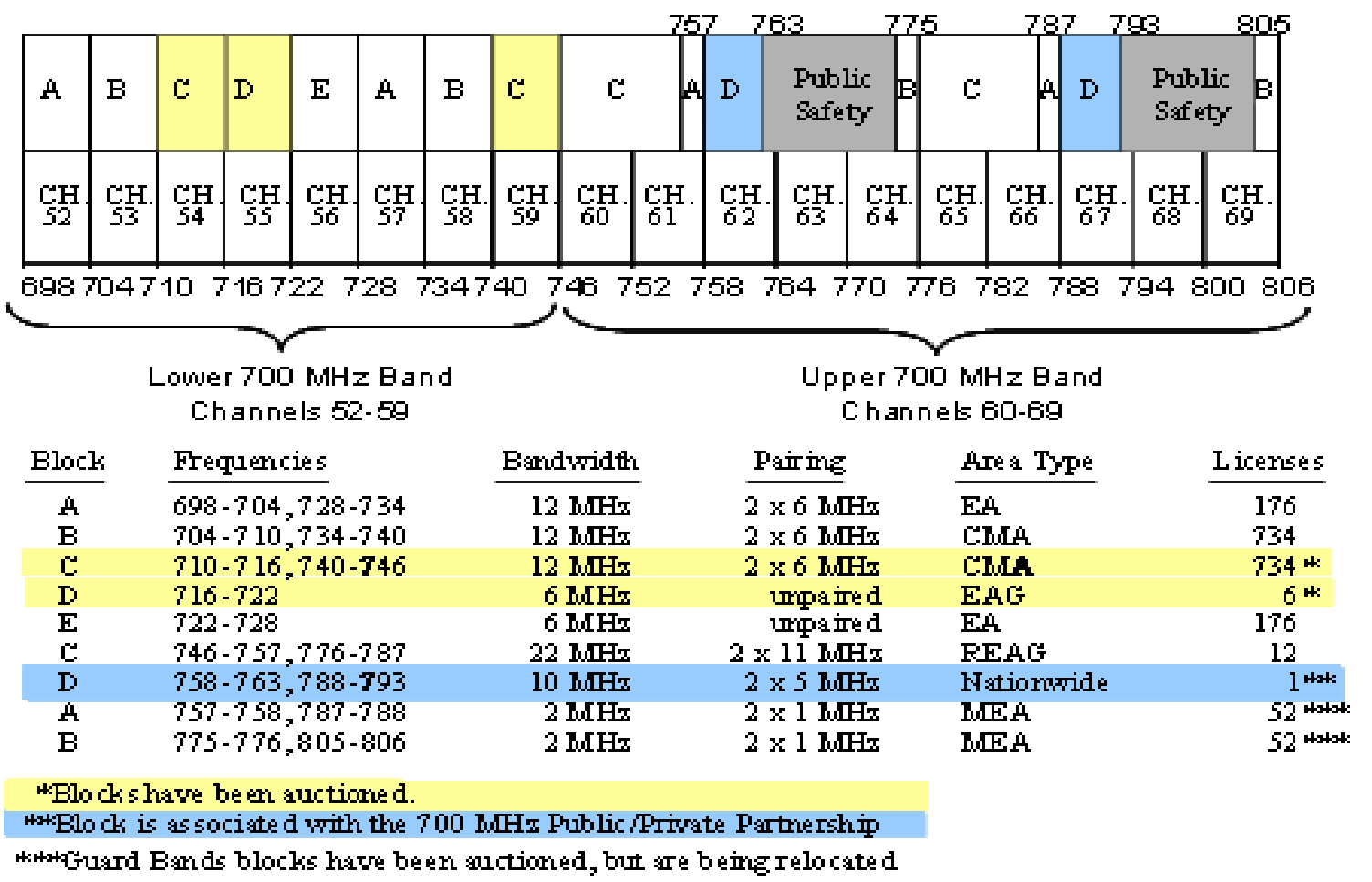

Figure 3-10: Planned Use of 700 MHz Band

\subsubsection{Audio Gateways}

Gateways provide an option for establishing connections between disparate communications by allowing devices of separate system and frequency types to communicate together. This is done by patching the different devices together using an audio gateway device. This technology is often used for emergency communications to enable interoperable communications between agencies. Figure 3-11, shows an example of a gateway that allows for three different communications networks to communicate with each other. Audio gateways provide the most flexible option for first responder communication by allowing dynamic creation of talk groups between different systems. They could also be used as configurable repeaters when deployed with a tower or aircraft.

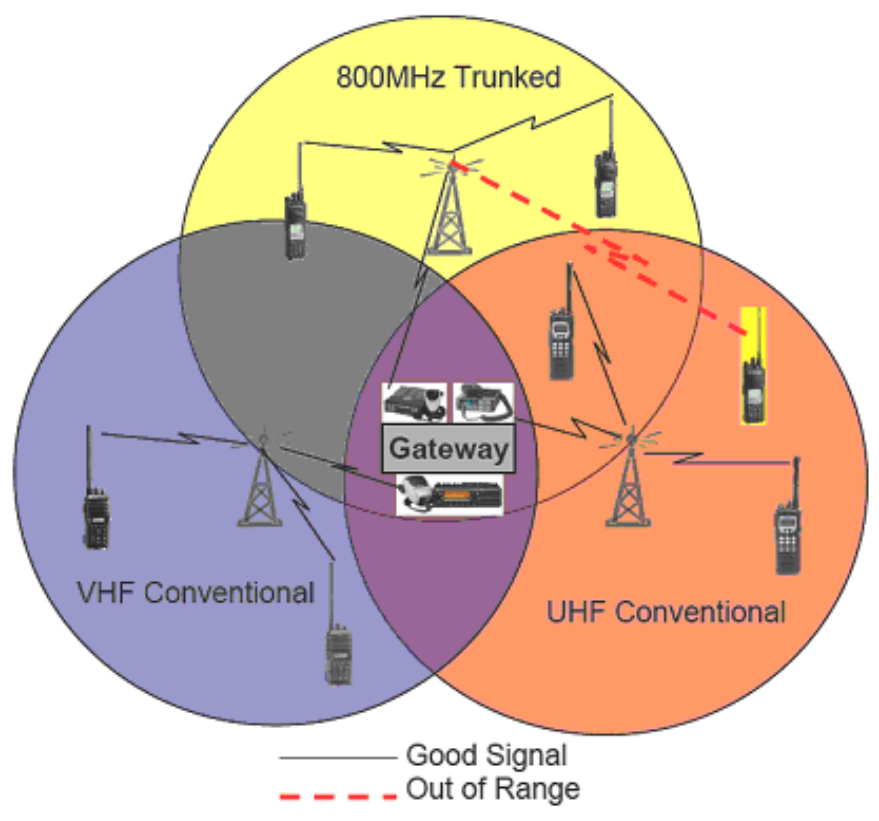

Figure 3-11: Example of Radio Gateway [53] 


\subsubsection{Software Defined Radio}

Software Defined Radio (SDR) provides a solution to interoperability by dynamically controlling operating frequency and radio link protocols through software. An example of an SDR system is the Joint Tactical Radio System (JTRS), which is the military version of SDR. These SDR radios will allow the user to communicate via satellite, CDMA, GSM and multiple types of LMR communication using an SDR. On April 14, 2006, the SDR forum had released a report titled Software Defined Radio for Public Safety, which provided recommendations to industry. [54] In this report the SDR forum gave a summary of recommendations to industry for SDR radio for public safety. Although, these systems have not been developed for the public safety market they show promise for providing interoperability for first responders.

\subsubsection{Commercial Cellular Devices and Service}

Cellular networks are the most widely deployed and most interoperable networks that are commercially available today. Devices and services can be purchased commercially off the shelf for fraction of the cost of LMR radios. Additionally, first responders will not need to maintain or upgrade their own infrastructure since the network operators do this already. However, cellular does have some drawbacks. Cellular networks are built for minimized cost not redundancy; therefore, network congestion would be more common on cellular network than on a private LMR network. In order for cellular networks to be successful in the first responder market, they must allow first responders to have priority access to voice and data. Some priority access is provided through Emergency Telecommunications Services.

\subsection{Emergency Telecommunications Services}

The Government Emergency Telecommunications Service (GETS) provides emergency access and priority processing in the local and long distance segments of the wireline Public Switched Telephone Network (PSTN). It is intended to be used in an emergency or crisis situation when the PSTN is congested and the probability of completing a call over normal or other alternate telecommunication means has significantly decreased. GETS is accessed through a universal access number using common telephone equipment such as a standard desk set, facsimile, modem, or wireless phone. A prompt will directs the entry of a PIN and the destination telephone number. Once authenticated as a valid user, the call is identified receives special treatment. [55]

Similar to GETS, Wireless Priority Service (WPS) was developed to provide priority for emergency calls made from cellular phones. WPS is a companion priority service with GETS and is an easy-to-use, add-on feature subscribed on a per-cell phone basis; no special phones are required. WPS is implemented as software enhancements to cellular networks, and is being deployed by cellular service providers in their coverage areas throughout the United States. [56] Although wireless voice and wireline voice have priority access with GETS and WPS, there is currently no governmental program which allows for prioritized data services. A data equivalent of WPS for wireless data would also need to be available for first responders to fully utilize the capabilities provided by cellular networks.

\subsection{Municipal WiFi Networks}

Where available, WiFi networks provide an option for wireless data connectivity. Most laptops purchased come with WiFi built in and WiFi hotspots can be established without any regards to radio licensing issues. WiFi access points are also relatively cheap to deploy. In 2006, Frost and Sullivan reported that 200 cities were operating municipal public safety WiFi networks with 600 new planned networks. However, since WiFi technology was intended to cover smaller areas rather than wide reaching areas, in 2007 many WiFi network operators have not been able to make the business profitable and will most likely shift the plans of Municipal WiFi deployments. Although a completely viable option for wireless data, network operators have yet to make these services profitable

\subsection{Summary and Conclusions}

The following is a summary and conclusions of the main points covered in this section: 
- E911 is an essential function of first responders that provides location of emergency callers.

- $\quad$ E911 comes in two different forms: wireline and wireless.

- Wireless E911 uses triangulation techniques or Assisted-GPS for locating the 911 caller determines which PSAP to route the caller to and reports the caller's location to the PSAP.

- NG 9-1-1 standards are being developed and architectures will support a multiplicity of IP based devices and multiple forms of media.

- Public safety wireless networks are primarily based on LMR technologies.

- The majority of public safety agencies in the U.S. purchase, own and maintain their LMR infrastructure, most of which is largely not interoperable with other agencies.

- There are two standards based LMR technologies P25 and TETRA. P25 is most popular in the U.S. and TETRA is more popular worldwide.

- There are a variety of interoperability solutions including, network patches, IP Core technologies, audio gateways and migration to cellular technologies.

- First responders can receive priority access public telephone networks using GETS and WPS services. 


\section{REFERENCES}

[38] Ragsdale, Billy, NENA Technical Liaison Chair, "9-1-1 Tutorial," PSAP CPE Technical Committee 9-1-1 Tutorial, http://www.nena.org/florida/Directory/911Tutorial\%20Study\%20Guide.pdf,

[39] Desourdis, R. I., Jr., D.R. Smith, W. D. Speights, R. J. Dewey, J. R. DiSalvo, "Emerging Public Safety Wireless Communication Systems," 2001, Artech House Publishers, ISBN 0-89006-575-6.

[40] Schulzrinne, Henning, "911 Services: Wireline, Wireless and VoIP," New York FCC Solutions Summit, March 18, 2004, http://www.cs.columbia.edu/ hgs/papers/2004/FCC-911-2004.ppt,

[41] Yilin Zhao, Motorola, Inc., "Standardization of Mobile Phone Positioning for 3G Systems," IEEE Communications Magazine, July 2002

[42] FCC, "Fact Sheet: E911 Phase II Decisions," October 2001, www.fcc.gov,

[43] U.S. Department of Transportation, "Next Generation 9-1-1 (NG 9-1-1) System Initiative System Description and High-Level Requirements Document," Version 1.1., Intelligent Transportation Systems, July 31, 2007.

[44] U.S. Department of Transportation, "Next Generation 9-1-1 (NG 9-1-1) System Initiative System Concept of Operations,” Version 2.0. Intelligent Transportation Systems April 6, 2007

[45] Hixson, R., B. Cobb, P. Halley, “9-1-1: The Next Generation NENA's Blueprint Steers 9-1-1 into the Future" 9-1-1 Magazine, January/February 2007.

[46] Swan Duncan, Associate Director Swan Communications, "Comparing TETRA with other Technologies", Presentation to TETRA Experience Dubai, November 27, 2006 , http://www.tetraassociation.com/uploadedFiles/Files/Presentations/Dubai06swancomparison.pdf

[47] National Task Force on Interoperability, "Why Can't We Talk, Working Together to Bridge the Communications Gap to Save Lives, A Guide for Public Officials," February 2003, http://www.safecomprogram.gov/NR/rdonlyres/322B4367-265C-45FB-8EEABD0FEBDA95A8/0/Why cant we talk NTFI_Guide.pdf,

[48] Davis, Tom, Chairman, "A Failure of Initiative: The Final Report of the Select Bipartisan Committee to Investigate the Preparation for and Response to Hurricane Katrina," http://www.katrina.house.gov/full_katrina report.htm,

[49] Kenyon, Henry S., “Secure Cellular System Serves Warriors and Rescuers,” Signal, January 2007.

[50] Lawlor, Maryann, Internet Protocol Network Protects Troop Convoys, SIGNAL, November 2006

[51] T Schofield, Teresa, "Dallas Launches City-Wide Interoperability Network," CoCo Communications Press Release, November 15, 2006, http://www.cococorp.com/pdf/News-DallasNetwork.pdf,

[52] FCC, "FCC Revises $700 \mathrm{MHz}$ Rules to Advance Interoperable Public Safety Communications and Promote Wireless Broadband Deployment," http://hraunfoss.fcc.gov/edocs public/attachmatch/DOC-275669A1.doc,

[53] Interoperable Communications Technical Assistance Program (ICTAP), Audio Gateway Handbook, March 2006, ICTAP-OG\&T-HNDBK-002-R0, http://www.npstc.org/documents/

[54] SDR Forum, "Software Defined Radio Technology for Public Safety," April 14, 2006, SDRF-06-A-0001V0.00, www.sdrforum.org 
[55] National Communication Systems, “Government Emergency Telecommunications Service” September 14, 2007 http://gets.ncs.gov/

[56] National Communication Systems, "Wireless Priority Service” September 14, 2007 , http://wps.ncs.gov/ 


\section{ACRONYMS}

$\begin{array}{ll}\text { ALI } & \text { Automatic Location Identification } \\ \text { APCO } & \text { Association of Public-Safety Communications Officials } \\ \text { DB } & \text { Data Base } \\ \text { DTV } & \text { Digital Act of 2005 } \\ \text { ESZ } & \text { Emergency Service Zones } \\ \text { GETS } & \text { Government Emergency Telecommunications Service } \\ \text { ICTAP } & \text { Interoperable Communications Technical Assistance Program } \\ \text { IETF } & \text { Internet Engineering Task Force } \\ \text { JTRS } & \text { Joint Tactical Radio System } \\ \text { LMR } & \text { Land Mobile Radio } \\ \text { MMS } & \text { Mobile Messaging Service } \\ \text { MSAG } & \text { Master Street Address Guide } \\ \text { NG 9-1-1 } & \text { Generation 911 } \\ \text { PSAP } & \text { Public Safety Answering Point } \\ \text { PSTN } & \text { Public Switched Telephone Network } \\ \text { SDR } & \text { Software Defined Radio } \\ \text { SRDB } & \text { Selective Router Database } \\ \text { TETRA } & \text { TErrestrial Trunked RAdio } \\ \text { VoIP } & \text { Voice over IP } \\ \text { WPS } & \text { Wireless Priority Service }\end{array}$




\section{Catastrophic Events and Telecommunications}

Catastrophic events or large disasters have unique effects on communications networks. The purpose of this section is to define those effects. Additionally, this section also examines worst-case scenarios in order to quantify the problem of providing a backup telecommunications system in the event of a catastrophic event or large-scale disaster.

\subsection{Emergency Communications}
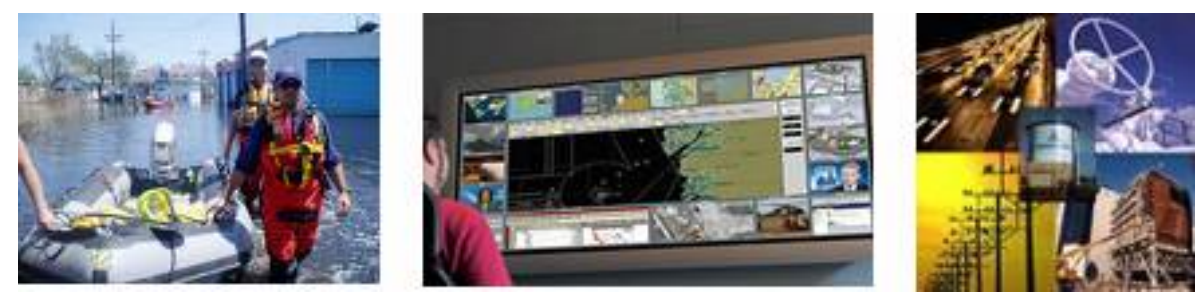

Figure 4-1: Emergency Communications

According to the Bipartisan Committee Investigation of Response to Hurricane Katrina, emergency communications needed during Hurricane Katrina could be grouped into three categories [57]:

\section{First Responder Communications}

Emergency Responders, Army, National Guard, Coast Guard, and Enhanced 911

\section{Command and Control}

Situational awareness, supply delivery, coordination of rescue and security forces, and transportation of evacuees

\section{Critical Infrastructure Restoration}

Clearing debris and restoring power and communication networks.

\subsubsection{First Responder Communications}

Fire, police, and emergency medical services Land Mobile Radio (LMR) systems were completely devastated by flooding and storm damage. Winds knocked down towers and water flooded backup generators. For three days, the police had no wireless communications. Once some communications were restored, three mutual aid channels were used between all first responders. Additionally, interoperable communications between federal, state, and local agencies could have been useful in coordinating rescue efforts. Enhanced 911 (E911), which reports the location of emergency calls, would have also been extremely useful when looking for evacuees. E911 is a vital capability that first responders rely on daily.

\subsubsection{Command and Control}

Commanders and state officers need to have situational awareness during a catastrophic event to make effective decisions. During Hurricane Katrina, reports from the media were used to determine situational awareness. These reports were grossly exaggerated and unreliable, as was evidenced from reports made about the Superdome. With a Public Safety Answering Point data fed into the Emergency Operations Center, commands could make decisions using information from citizens who are on scene. Listening to the 911 calls of the local citizens can greatly enhance the situational awareness of the on-scene commander.

Officers also need geo-location capabilities for emergency forces and evacuees. This information would be especially useful if overlaid on geo-spatial imagery so that important decisions could be made quickly. Many 
commercial cellular devices come with GPS capabilities and using cellular data networks forces could be tracked and directed appropriately.

\subsubsection{Critical Infrastructure Restoration}

In addition to search, rescue, and relief efforts, critical infrastructure must be restored so that water, roads, and communications can be made available. Personnel executing this restoration mission also require communications after a catastrophic event. Additionally, these communications must become available immediately after the event so that the restoration process can begin. Restoring basic services as soon as possible will relieve the suffering of evacuees by fulfilling their basic needs and reduce the amount of supplies that will need to be transported to evacuees.

\subsection{Cellular Communication Outage: Hurricane Katrina}

On August 29, 2005, Hurricane Katrina made landfall onto Louisiana and Mississippi causing widespread destruction. This was the first time a catastrophic event of this magnitude had struck the U.S. The hurricane virtually destroyed every form of communication available, everything from wireline phones, LMRs, and cellular phones were destroyed. Because of its impact to the telecommunications networks, Katrina serves as an ideal case for understanding a worst-case telecommunications outage, in particular wireless communications. "A Failure of Initiative: The Final Report of the Select Bipartisan Committee to Investigate the Preparation for and Response to Hurricane Katrina" summarized the communications outage that occurred during Hurricane Katrina. This was the primary resource for data concerning the communications outages during Hurricane Katrina.[57]

\subsubsection{Cellular Communications}

New Orleans suffered devastating damage to their cellular communications infrastructure. In two cases, entire networks where taken out of service due to critical damage. These outages occurred with operators Verizon and Cingular Wireless (now AT\&T Mobility). Combined, Verizon and AT\&T, the two largest operators in the U.S., have over $50 \%$ of the entire U.S. market share. Verizon lost a major connectivity through a fiber ring that was ran by Bell South. [58] This loss of connection caused loss of cellular service to the entire area. Cingular lost a Mobile Switching Center (MSC) due to flooding. [4] Exacerbating these major system failures, were multiple cases of damage to the physical towers or Base Transceiver System (BTS) equipment. Power was also a major issue, generators were stolen or could not be refueled due to access and security reasons. [69]

As shown in Figure 4-2: BTS Outage during Hurricane Katrina, approximately $70 \%$ of the BTSs, that provided wireless cellular service in New Orleans were Out of Service (OOS) after Katrina. [58] There was no data available in the 2-week timeframe after the hurricane hit; in some cases operators protected this data for proprietary reason. Therefore, the curve in the blue box has been interpolated for a worst-case scenario. 


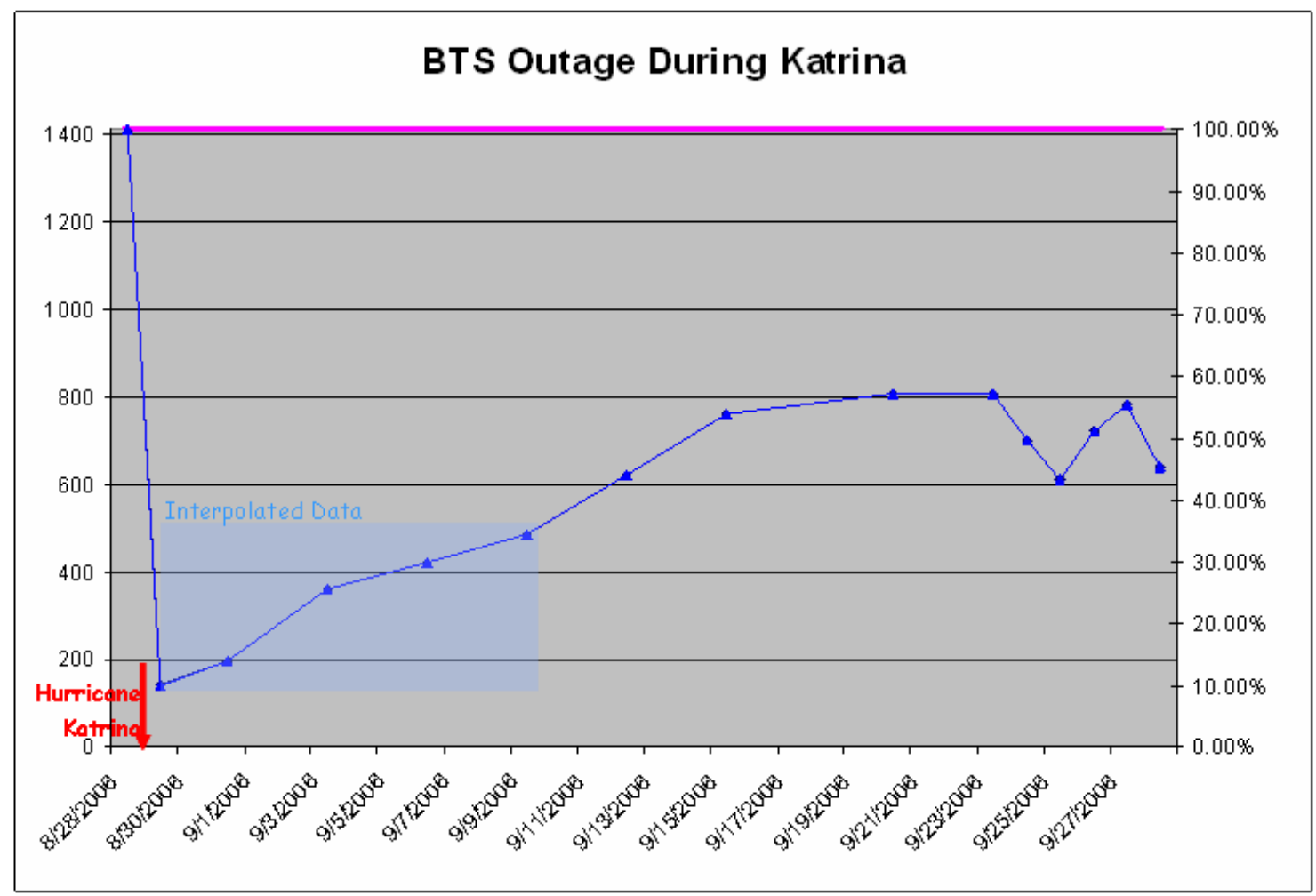

Figure 4-2: BTS Outage during Hurricane Katrina

\subsubsection{Cellular System Outage Scenarios}

In this study, cellular networks are a main portion of the analysis since these networks are most widely deployed and interoperable networks. Although these scenarios were drawn from cellular outage, analysis for LMR networks would be very similar.

\section{- $\quad$ Physical Damage to BTS}

The BTS includes the tower, antennas, microwave dishes, enclosures with cellular equipment, and in some cases a power generator. Any damage to these components could cause the BTS to go OOS. This is shown in Figure 4-3: BTS Outage Scenario with the red "x." During Hurricane Katrina, sectors that faced the wind directly were damaged due to flying debris. In this case, only portions of the cell would have service.

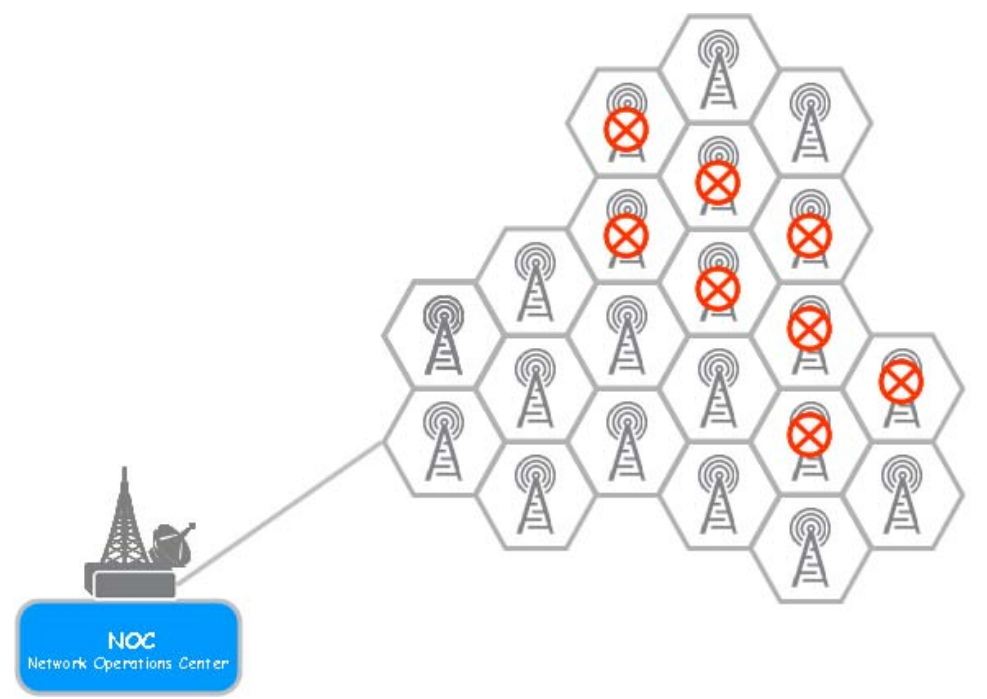

Figure 4-3: BTS Outage Scenario 


\section{- $\quad$ Loss of BTS Backhaul}

BTS backhaul is the link between the BTS and the Mobile Switching Center (MSC) and BTS System Controller (BSC), which is contained in the Network Operations Center (NOC) or Mobile Telephone Switching Office (MTSO). The backhaul could be a T1 using a physical cable (fiber/copper) or could be a microwave signal. Without this link, calls will not be served within the BTS cell. Additionally, the BTS may still be radiating a pilot channel so phones with mobiles within the cell may still show signal strength from the BTS. Physical damage can cut or damage cables causing a loss of backhaul. Damage to or misalignment of microwave dishes on the BTS tower could also cause a loss of backhaul. The Figure 4-4: BTS Backhaul Outage Scenario shows physically damaged BTSs (red x) and BTSs, which are OOS since their backhaul (yellow x) had been damaged.

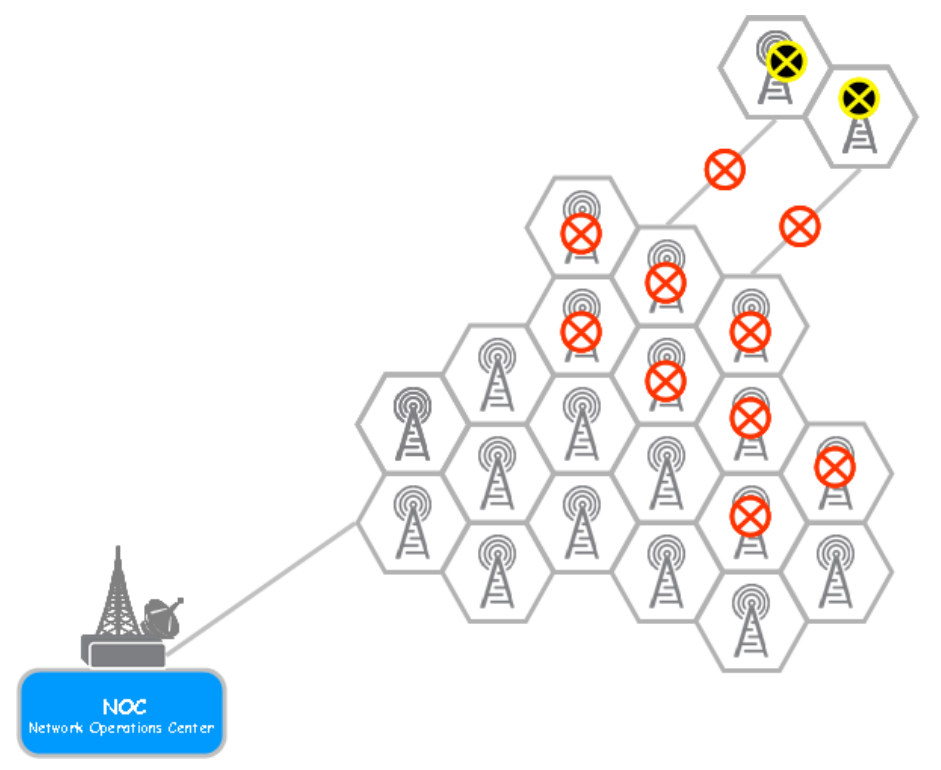

Figure 4-4: BTS Backhaul Outage Scenario

\section{- Physical Damage to NOC/MTSO}

The NOC/MTSO is the building that contains the MSC and BSC equipment. This equipment could contain equipment that controls 200 BTSs or more. OOS equipment in this building can cause a major geographic outage. Figure 4-5: MSC Outage Scenario shows that BTSs with the yellow x could still be transmitting however may not be able to provide service.

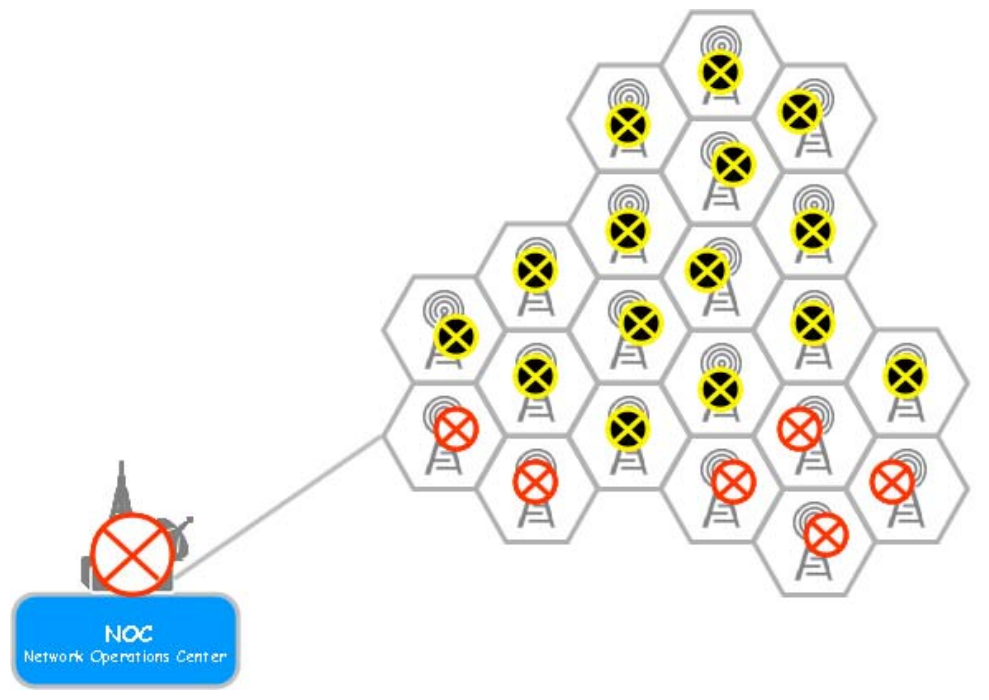

Figure 4-5: MSC Outage Scenario 


\section{- $\quad$ Loss of Backhaul to Public-Switched Telephone Network (PSTN)}

Usually these links are T1s, over fiber or copper, provide call control and also a call path to the telephone network. Without this connection to the PSTN calls can only be routed to calls within the network. Figure 4-6: PSTN Backhaul Outage Scenario shows how the whole network could be out of service or in some cases only make an in-network call.

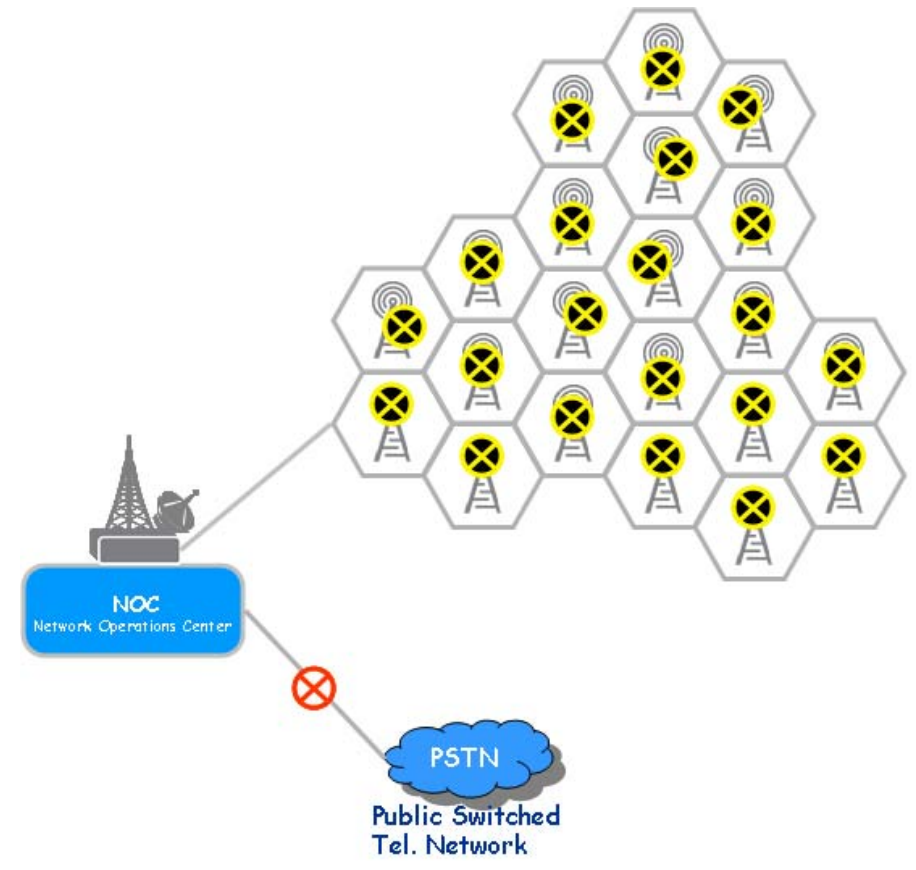

Figure 4-6: PSTN Backhaul Outage Scenario

- Loss of Power

Many, but not all BTSs have backup generators. Typically, backup generators on BTSs have 48 hours of backup power. Equipment inside the NOC/MTSO will also have a backup generator.[59] Figure 4-2: BTS Outage during Hurricane Katrina shows 4 BTSs with the blue $\mathrm{x}$ that are OOS due to lack of power.

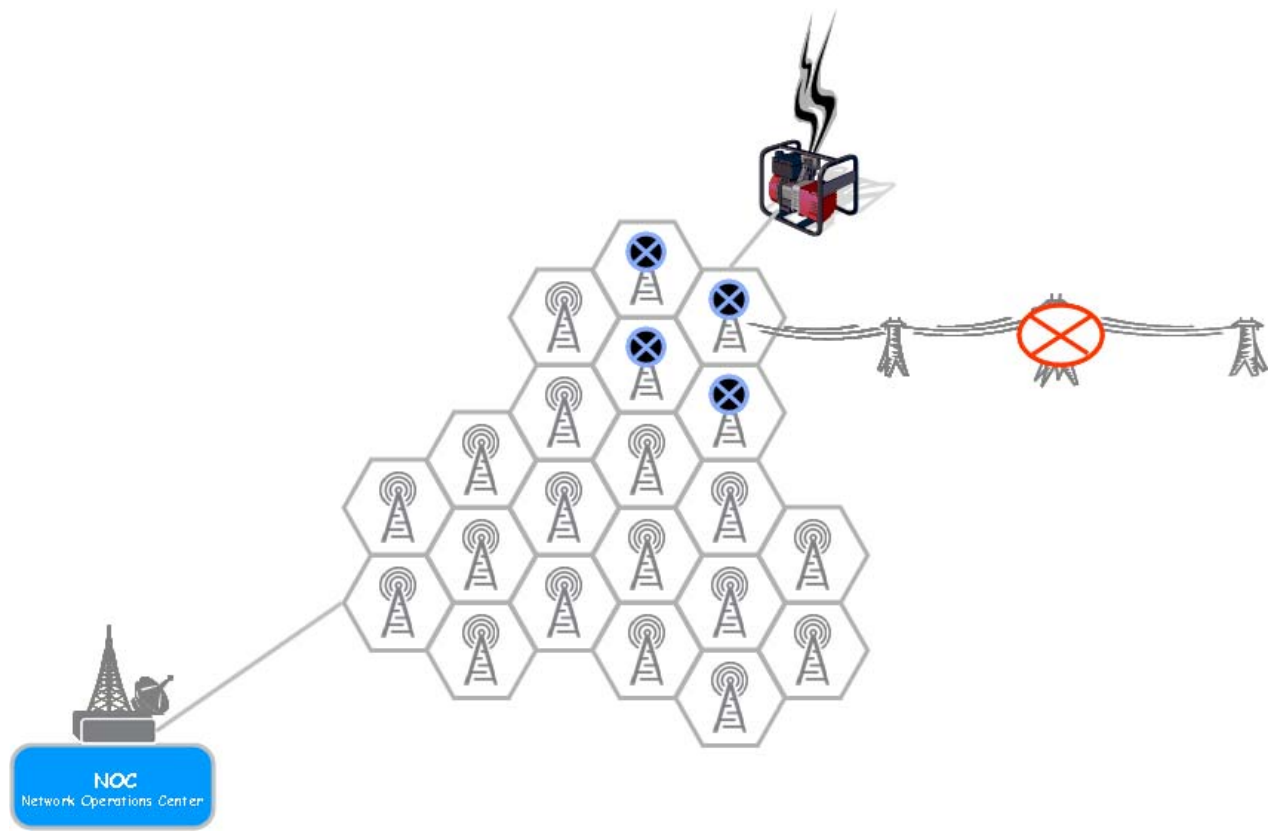

Figure 4-7: Power Outage Scenario 


\subsection{During Catastrophic Event}

Following Katrina, 38 PSAPs were severely debilitated and/or destroyed, even if someone had a working phone they could not call 911. PSAPs were destroyed, disconnected from the network or abandoned. Unfortunately, 911 is a critical service for first responders to identify where incidents are. Without this capability, people felt abandoned or ignored and first responders had to go door to door to find evacuees. [65] During a catastrophic incident, it is assumed that terrestrial 911 systems will fail and backup systems are needed.

\subsubsection{Call Traffic Patterns}

Normal 911 call traffic patterns are very similar to regular voice call traffic patterns. Daily patterns include reasonably predictable peaks and lows during the day. However, aberrant situations, such as natural disasters or wartime attacks have a much different affect on the 911 networks. Although there is not much data from Hurricane Katrina, due failure of the network, analyzing similar events gains an understanding of how networks might behave. One example is the Israeli and Hezbollah conflict during July and August 2006. Although the conflict of Hurricane was more destructive in nature, this conflict had a comparatively sized area of impact and number of deaths. MTC Touch Lebanon, the local cellular operator, released a report on June 2007 that highlighted the impacts of the war on to their cellular network, in particular the number of 112, U.S. equivalent of 911, emergency calls that occurred during that period. [60] This is shown in Figure 4-8.

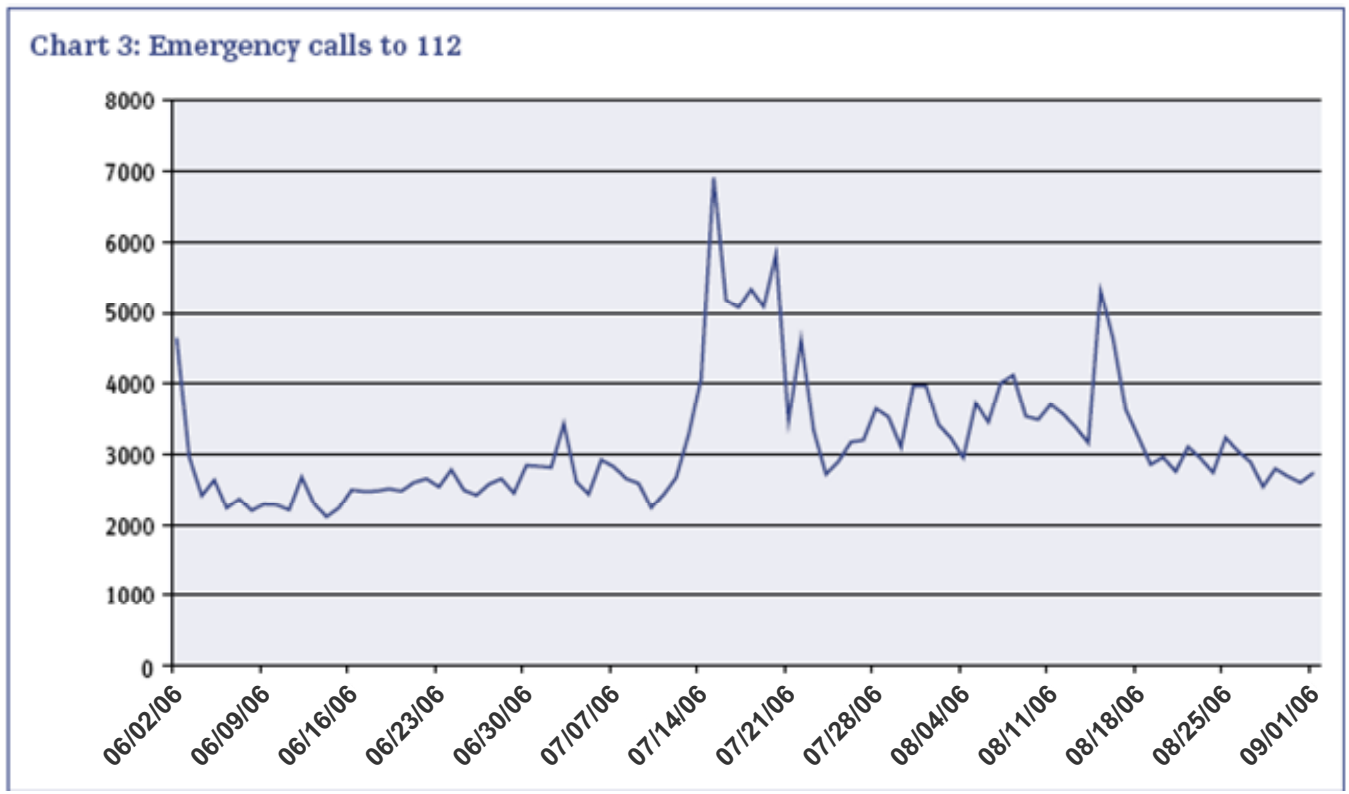

Figure 4-8: Emergency Calls during Israeli Hezbollah Conflict

The peak traffic occurred on July 14, 2006 with 6,883 total calls. It is speculated that an immediate drop in 112 call volumes after July 14 was due to due to evacuations of the area. Later peaks are seen that correlate to events of bombing and attacks to Lebanese cities. Observing these trends, once the event occurs, there is a significant surge in traffic of approximately two to three times the average daily amount. Additionally, for a short time an elevated rate of traffic was sustained for 1 week, followed by peaks correlated to other related events or secondary effects from attacks.

From anecdotal data, similar patterns were described. Rear Admiral, Robert F. Duncan, commanding officer of the Coast Guard during Hurricane Katrina, described that some people did not need immediate evacuating from the aftermath of Katrina. They were able to stay for period of 2-3 days until their water or food stores were exhausted. Others were injured and needed immediate medical attention. This scenario described would have shown a similar pattern, first a sharp elevated peak for a period following the disaster and secondary peaks following from those who ran out of food or water. 
In all cases shown, during a catastrophic event, many emergency calls will come within the same period. Answering an excessive amount of calls within a very short period could be limited to the number of PSAP operators that can answer the calls. It would also seem prudent that the most important information from a 911 emergency call during a catastrophic event is the caller's location and then the severity of their emergency. Therefore, 911 automatic answering capabilities are desirable when dealing with a catastrophic event emergency 911-call traffic to mitigate the amount of simultaneous traffic and expedite distress locations.

\subsubsection{Automatic 911 Answering}

In the simplest example, when a 911 call is made in current systems its location and phone number are sent to the PSAP. Instead of answering the call, a text message could be sent to the user notifying them that their location was received and that help is on the way. It could also allow the caller to send more information via SMS, rather than a call, to minimize network loading. Furthermore, the PSAP could call the 911 caller if their case was severe. This example could be done today with no changes to the mobile device; however, additional capabilities at the PSAP would need to be developed.

If first responders have additional capabilities on their mobile devices this system and method could use this information to direct first responders to the incident. In addition to the automatic 911 answering capabilities, the system could also track first responder locations and correlate them with 911 callers. This method could be a more efficient method for determining locations of 911 callers. Applications of this nature could easily be downloaded to a first responder mobile device that could be used to provide the 911 information from the PSAP to first responders in replacement of a dispatch function.

\subsubsection{Worst-case 911 Call Volumes}

Although, the effects of Hurricane Katrina were devastating, early warnings made it possible to evacuate $90 \%$ of New Orleans minimizing the loss of life. Worst-case disasters, such as an earthquake or a terrorist attack, come with no warnings. Therefore, planning for these worst-case scenarios is necessary for estimating the maximum load on an emergency 911 network. According to The Homeland Security Council's disaster planning scenario, the worst-case scenario is for a 10-kiloton improvised nuclear device. It is estimated that in this scenario there will be 450,000 evacuations/displaced persons. [70]

In this estimate, some basic assumptions have been made. First, the call pattern from this disaster will be very similar to the call patterns discussed. Second, once a 911 call is made there will be no repeats from the same user. This second assumption is made from simplicity in the analysis. Third, daily 911 call patterns during the initial 24-48 hours will be constant and result in network full loading, with elevated daily call volumes continuing for up to 1 week. Finally, it is assumed there would be approximately 450,000 911 callers in the worst-case scenario. If these assumptions and the call history from the Hezbollah/Israeli conflict are used, an estimate can be made (shown in Figure 4-9). 


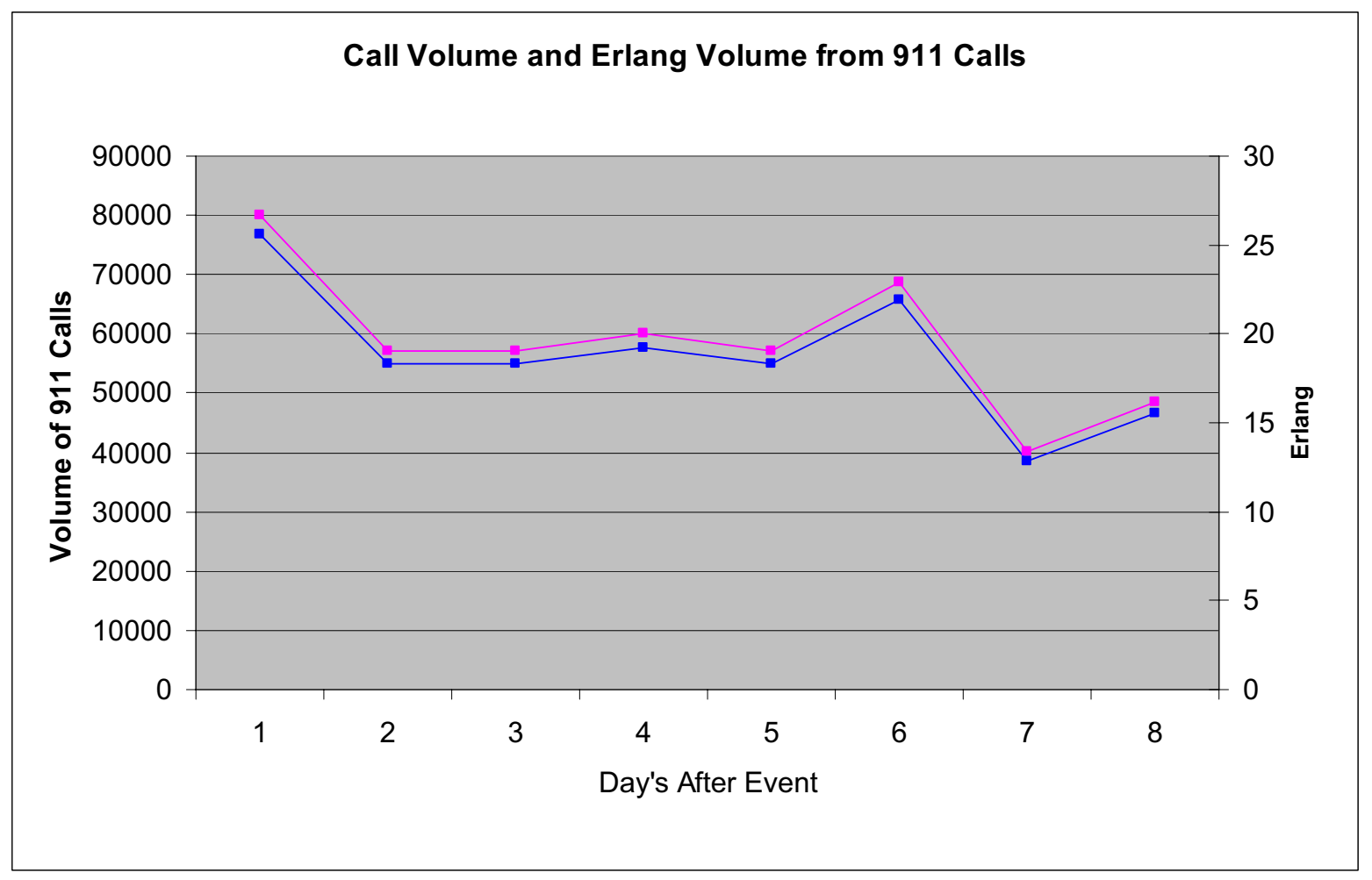

Figure 4-9: Worst-Case 911 Call Estimate

Although the call volume for 911 calls is very high, the contributions from all calls are very small. Since the average call hold time for a 911 call is 30 seconds, the amount of Erlangs contributed on a daily basis are very low in comparison with other traffic. [71] Therefore, accepting 911 calls on a regular network should be negligible. However, this is assuming that users make a single 911 call and do not retry again once they have made the call. Additionally, a system that only allows 911 calls will not need to support very many simultaneous channels. In this case, a system that supports 30 simultaneous channels would be more than adequate to support 911 calls in a worst-case disaster situation.

\subsection{Emergency Cellular Communication System}

\subsubsection{Estimates for Daily Call Volumes}

In order to quantify the amount of communications that should be used to support a disaster scenario, it is important to understand traffic patterns during the disaster, and then plan the system capacity towards the maximum capacity system. Figure 4-10, shows what a potential traffic pattern could look like based on user population data and outage periods during Hurricane Katrina. In Figure 4-10, the right Y-axis represents the percent of cell sites in service. The blue line shows the actual and estimated amount of cell sites in service after Hurricane Katrina. 


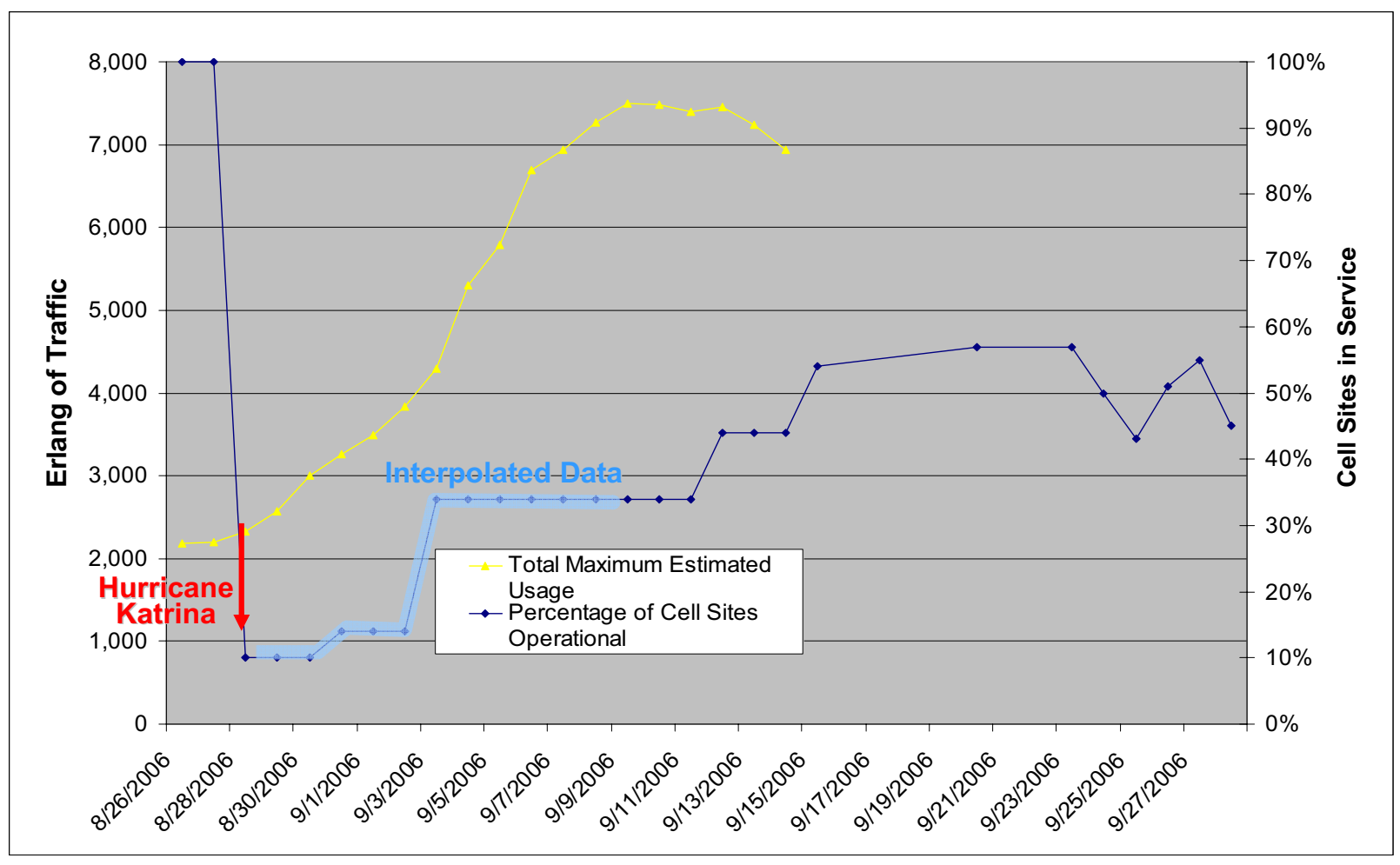

Figure 4-10: Estimated Daily Call Volumes

In Figure 4-10, the left Y-axis represents Erlangs. The yellow line is the summation of 911 calls and priority user traffic. 911 calls were estimated to be 30 seconds long and volumes were estimated using Figure 4-9. Their contributions to the total volume were nominal. Priority user traffic was estimated from populations of military, first responders and public utility, which served during Hurricane Katrina. [57][62] Out of the total military population, each commanding officer of each military echelon was estimated to need a mobile device. Fifty percent of first responders and public works were estimated to each need a mobile device. All users were given a conservative 0.2 Erlang.

Figure 4-10 reveals important information about large-scale disasters. First and most obvious is the need for emergency communications immediately after the disaster, an ideal placement for a continuously on-station Airborne Communication Node (ACN). Second, catastrophic affects on the telecommunications networks are persistent. Large scale network outages could be expected to last weeks or even months. Third, traffic estimations show an immediate need for 911 emergency calling capabilities and a capability to support a significant amount of users on a private network.

\subsubsection{Estimation of Daily Maximum Load}

A complete coverage strategy between deployable terrestrial assets and the ACN is important since use of an $\mathrm{ACN}$ will be most effective up to 2 weeks after the disaster occurs. This is due to the following reasons. First, emergencies after disasters require immediate attention in order to minimize loss of life. Second, the average maximum standby battery life of a cell mobile device will last 300 hours. [63][64] Additionally, it is estimated that terrestrial deployable assets will begin to be deployed within 48-72 hours after the event occurs.

Following this initial ACN window, there will be a period of time where both the terrestrial assets and the ACN could be simultaneously deployed. There may be areas that have experienced flooding, debris or security issues where an ACN can cover where terrestrial assets cannot. After $\sim 300$ hours, cellular emergency calls will most likely not occur in the ACN coverage areas, because all mobile device batteries will be completely depleted at this time. Also at this time, evacuees will be moved into recovery areas where terrestrial assets can be deployed. However, the ACN capability could still be used in areas where search efforts and infrastructure restoration efforts are occurring. This overall process would require coordination between various different government and 
commercial agencies. Figure 4-11 shows the ACN and terrestrial effectiveness windows pictorially. Within the ACN effectiveness window (Figure 4-11), the maximum daily traffic peak is estimated 4,200 Erlang of traffic. This is primarily due to the increasing population of priority users.

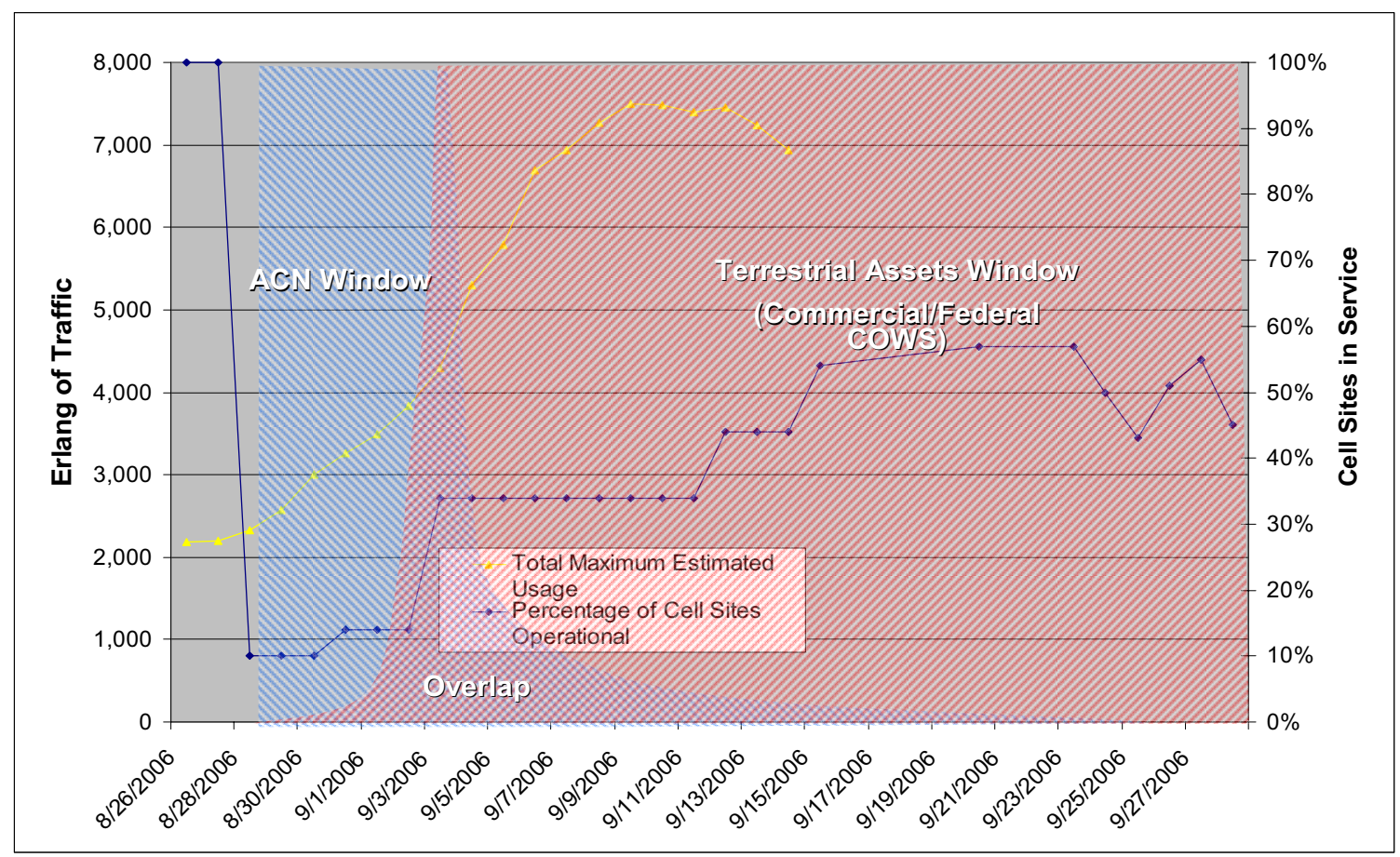

Figure 4-11: ACN and Terrestrial Assets Effectiveness Windows

\subsubsection{Estimated Daily Call Trends}

Within the ACN effectiveness window a total daily maximum traffic peak is estimated at 4,200 Erlang. Using daily maximum traffic and estimation of the hourly call trends, it is possible to determine the number of needed simultaneous channels for the emergency response system. There are two assumptions that can be used for daily traffic patterns: daylight search and rescue efforts, and a continuous rescue effort.

In the daylight rescue case, call traffic patterns will likely be very similar to normal patterns, but shifted in time. Using 4,200 daily Erlangs load and applying a normalized high-traffic day with hourly trend shifted in time, Figure 4-12 can be derived. From this estimate, the standard Erlang B table can be used to calculate the number of simultaneous channels needed to support the hourly peak with a Grade of Service of $1 \%$. This yields $\approx 350$ simultaneous channels. 


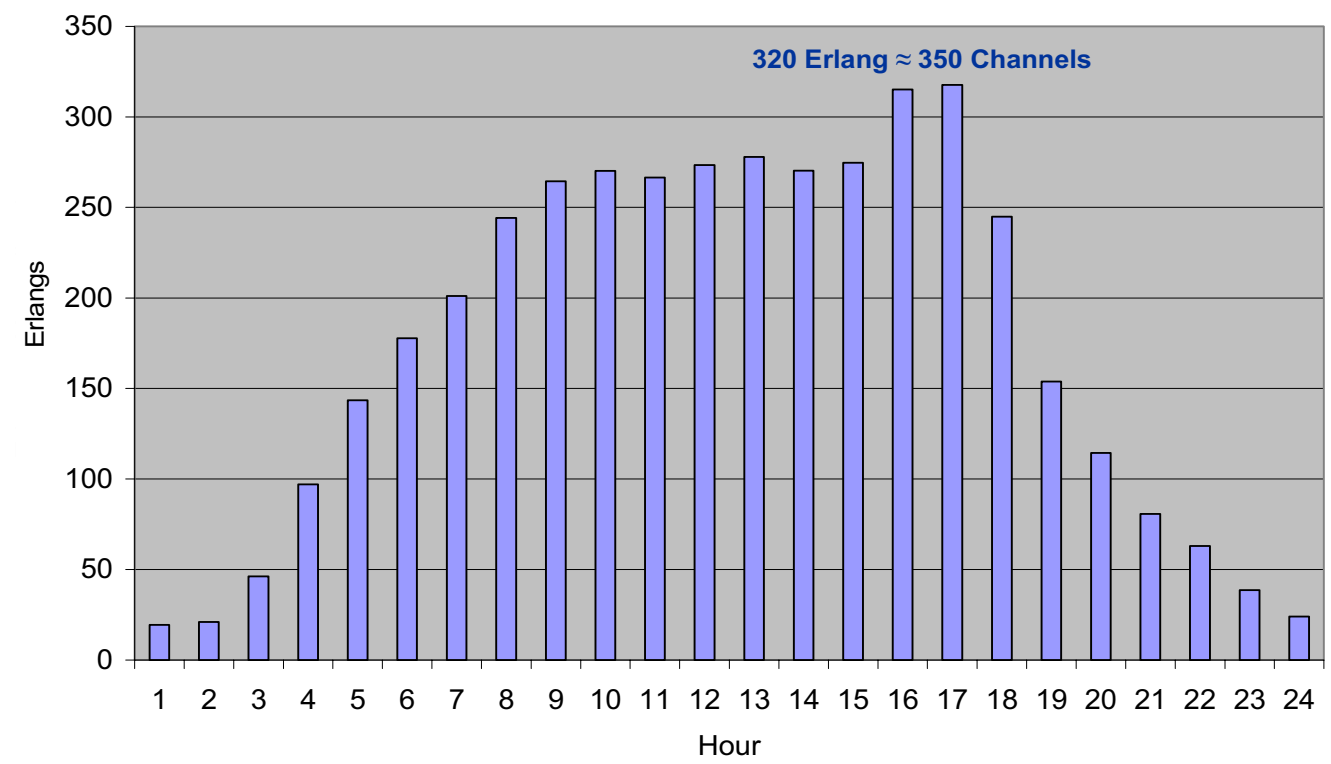

Figure 4-12: Estimated Call Load Daylight Rescue

In the continuous rescue case, 8-hour rescue shifts were assumed and a normalized traffic curve was estimated. [65] Using this estimate and amount of total daily traffic, this yields Figure 4-13. Based on this call load model, the estimated Erlang peak was 187 Erlang, which yields 207 simultaneous channels needed from the Erlang B table. In discussions with integrators of the deployable cellular systems used by federal agencies, a payload of $\approx 1000 \mathrm{lb}$ could support 300 simultaneous calls using commercial off-the-shelf equipment.[66]

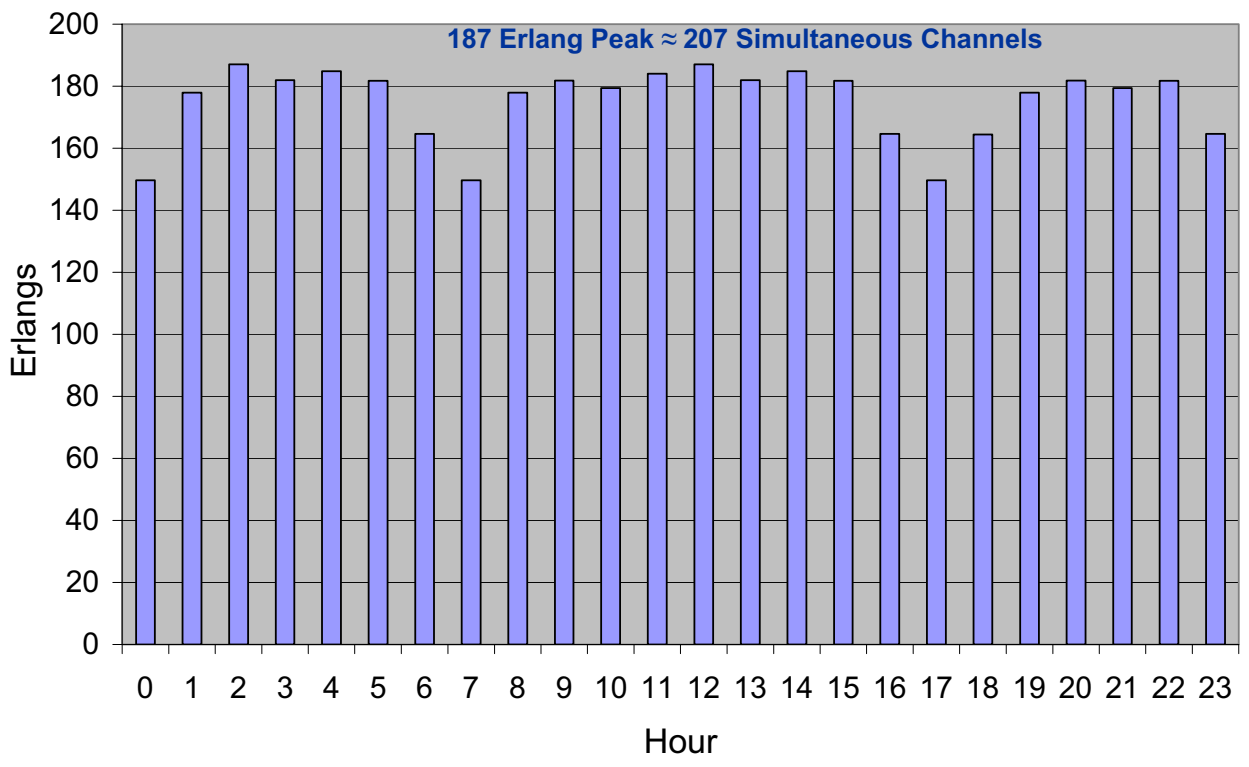

Figure 4-13: Estimated Call Load Continuous Rescue

\subsubsection{Backhaul Estimates}

Most cellular systems use T1 (E1 in Europe and Asia) links as the means to provide backhaul communications between the base stations and NOC. The T1s are typically microwave links or some form of fiber lines that carry signaling, data, and control messaging that are vital in the operation of the cellular system. A T1 contains 24 channels, and a total bandwidth of $1.544 \mathrm{Mbps}$. Typically there are up to four T1s deployed at a base station, compressed at a 4:1 ratio, but there can be more depending on the user load and configuration of the base station. 
Since the commercial cellular systems use the T1 standard, the ACN analysis will follow those parameters for planning the backhaul or reach-back capability. As previously mentioned, the bandwidth of a T1 is $1.544 \mathrm{Mbps}$, or 24 channels and a single base station can typically connect to four T1s, which would give it a total capacity of 96 channels. For the ACN, this would imply a capacity of 96 simultaneous out of network calls, or the capacity of one commercially available base station.

Given the expected high utilization of the backhaul, and the expected population density of nearly 300 simultaneous users, the bandwidth of the backhaul would be close to $20 \mathrm{Mbps}$, or $13 \mathrm{~T} 1$ links (uncompressed). Using a 4:1 compression could mean that only three T1s would be required for the backhaul. In addition to compressing the T1 lines, the cellular systems could be configured such that backhaul connectivity is limited. For example, if only half of the traffic is allowed to connect with the PSTN, two T1s could be required once they are compressed. This is only for cellular systems and does not include any LMR, video, or data transfers that may also be required.

In addition to using the backhaul to maintain communications with the PSTN and PSAP, the backhaul would be used to send tactical data to key stakeholders such as the Federal Emergency Management Agency (FEMA), the Department of Homeland Security (DHS), or even the president of the United States. The information could be anything from simple voice to streaming video of the area of interest. The resulting increase of bandwidth for these applications would also need to be taken into account when planning the capacity of the backhaul.

\subsection{Estimates for an Airborne Wireless Data Network}

Wireless data networks are beginning to augment existing first responder networks. The capabilities offered from a wireless broadband network can greatly assist public safety workers in offering additional capabilities with geo-location information and an exchange of many different types of media between responders. Traffic planning of these networks is a very difficult problem, due to the self-similar nature of data traffic.[67] Additionally, there has been little existing research done on first responder data traffic. Furthermore, next generation first responder applications have not yet fully come into fruition. In general, wireless data traffic engineering is still being developed and methods for doing network sizing are still esoteric. In order to properly size, a network of models will need to be developed and simulated to overall determine traffic patterns.

However, the SAFECOM program of the Department of Homeland Security, released a Statement of Requirements (SOR) for Public Safety Wireless Communications and Interoperability. [68] This SOR provides requirements for network providers for use cases as well as network performance specifications for first responders. The SOR data services used by first responders can be summarized in Table 1-1.

Table 4-1: First Responder Data Applications

\begin{tabular}{|c|c|c|}
\hline Service & Description & Service Class \\
\hline Push to Talk Voice & $\begin{array}{c}\text { Common Net Traffic used in current } \\
\text { LMR Systems }\end{array}$ & Conversational \\
\hline Status Data & $\begin{array}{c}\text { Periodic updates to centeralized data } \\
\text { base for biometrics, GPS Location Info, } \\
\text { Equipment Data }\end{array}$ & Background \\
\hline Instant Messaging & Text Messages between responders & Interactive \\
\hline Streaming Video/Data & Ground based or Aerial & Streaming \\
\hline E-mail & E-Mail w/ Attachments & Background \\
\hline Web Browsing & $\begin{array}{c}\text { Access to Maps, Geolocation Data, } \\
\text { Building Layouts, Medical and Criminal } \\
\text { information }\end{array}$ & Interactive \\
\hline
\end{tabular}

Services classes are based on $3^{\text {rd }}$ Generation Partnership Project (3GPP) standards for treatment of the data. In general, they can be seen as the following. Streaming class includes streaming audio and/or video where delay 
and jitter must be low. Data integrity is not as critical. The interactive class includes Web browsing and database retrieval. For interactive classes of traffic, data integrity is more important than delay. Background class applications include download of e-mails and file transfers. There is no strong delay or jitter requirements and data integrity is critical. These classes generalize the services that the first responders will require. [67]

\subsection{Summary and Conclusions}

Based on the analysis presented the following conclusions could be reached:

- There are 3 critical forms of communication

1. First Responder Communications

2. Command and Control

3. Critical Infrastructure Restoration

- During a catastrophic incident, all forms of terrestrial communications will be either completely destroyed or severely debilitated.

- During a catastrophic incident, 911 call loading will experience severe peaks, however due to the low call hold time 30 simultaneous channels should be adequate for a worst case scenario

- During a catastrophic incident, 911 call volumes will be very high this may require an alternative answering technique rather than a PSAP operator

- The ACN will be most effective for a two-week period immediately following the incident this is due to limited battery life and availability of terrestrial assets.

- Based on the calculated estimates 350 simultaneous channels are needed for a daylight rescue and 207 simultaneous channels are needed for continuous rescue case a payload of $1000 \mathrm{lbs}$ could support 300 simultaneous channels using COTS technologies. 


\section{REFERENCES}

[57] Davis, Tom, Chairman, "A Failure of Initiative: The Final Report of the Select Bipartisan Committee to Investigate the Preparation for and Response to Hurricane Katrina," February 15, 2006, http://www.katrina.house.gov/full_katrina_report.htm

[58] Martin, Kevin J., Chairman, Federal Communications Commission, "Hearing on Public Safety Communications from 9/11 to Katrina: Critical Public Policy Lessons Before the Comm. on Energy and Commerce, Subcomm, 109th Cong," September 29, 2005, http://energycommerce.house.gov/108/hearings/09292005Hearing1648/Martin.pdf

[59] Oberg, Chris MTS Network Security/Network Integrity VXW NCC Rep, Personal Interview November 2006.

[60] MTC Group, Mobility, “A Nation Under Siege,” June 2007, www.mtctelecom.com

[61] The Department of Homeland Security, "National Response Plan," December 2004, http://www.dhs.gov/xprepresp/committees/editorial 0566.shtm,

[62] Smith, Marc,Public Information Officer State of Louisiana, Interview, November 6, 2006.

[63] Krusell, “The Best Selling Mobile Devices in 2006," January 19, 2007.

[64] GSM Arena, Standby Power Statistics Various Mobile device Types, October 2007, http://www.gsmarena.com/

[65] Duncan ,Robert F., Rear Admiral, U.S. Coast Guard, Interview, November 15, 2007.

[66] Tackett, James, VP Homeland Security, Rivada Networks, August 23, 2007.

[67] Ribeiro, Leila Z., Dasilva, Luiz A., "A Framework For The Dimensioning Of Broadband Mobile Networks Supporting Wireless Internet Services,” IEEE Wireless Communications, June 2002.

[68] SAFECOM of the Department of Homeland Security, "Statement of Requirements (SOR) for Public Safety Wireless Communications and Interoperability," January 26, 2006, http://www.safecomprogram.gov/SAFECOM/library/technology/1258 statementof.htm

[69] Lawson, Stephen InfoWorld, "Cell carriers tackle Katrina damage," IDG News Service September 1, 2005, http://www.infoworld.com/article/05/09/01/HNcarrierskatrina_1.html,

[70] Howe, David "Planning Scenarios, Executive Summaries, The Homeland Security Council," July 2004, http://www.globalsecurity.org/security/library/report/2004/hsc-planning-scenarios-jul04 exec-sum.pdf

[71] Musgrove, Peter, AT\&T Wireless, Personal Interview, January 2007. 


\section{ACRONYMS}

$\begin{array}{ll}\text { 3GPP } & 3^{\text {rd }} \text { Generation Partnership Program } \\ \text { ACN } & \text { Airborne Communication Node } \\ \text { BSC } & \text { BTS System Controller } \\ \text { BTS } & \text { Base Transceiver System } \\ \text { COLT } & \text { Cells on Light Truck } \\ \text { COW } & \text { Cell on Wheels } \\ \text { DHS } & \text { Department of Homeland Security } \\ \text { E911 } & \text { Enhanced 911 } \\ \text { FEMA } & \text { Federal Emergency Management Agency } \\ \text { LMR } & \text { Land Mobile Radio } \\ \text { MSC } & \text { Mobile Switching Center } \\ \text { MTSO } & \text { Mobile Telephone Switching Office } \\ \text { NOC } & \text { Network Operations Center } \\ \text { OOS } & \text { Out of Service } \\ \text { PSAP } & \text { Public Safety Answering Points } \\ \text { PSTN } & \text { Public-Switched Telephone Network } \\ \text { RF } & \text { Radio Frequency } \\ \text { SMS } & \text { Short Message Service } \\ \text { SOR } & \text { Statement of Requirements } \\ \end{array}$




\section{Options for Architectures and Restoration Techniques}

The purpose of this section is to define the different architectures, components, and techniques used to provide critical communications from an Airborne Communications Node (ACN). This section provides basic analysis for the different architecture possibilities while listing comparative advantages and disadvantages of each. This section begins with defining the scope by referring to the generalized basic elements. These basic elements are then explained further by examining different options for using aircraft for restoration techniques, architectures for each technique, and potential commercial off-the-shelf (COTS) equipment. The section is concluded with an ideal emergency communications architecture.

\subsection{Generalized Basic Elements}

Figure 5-1 lists the general basic elements that make up the architecture of the airborne communications node.

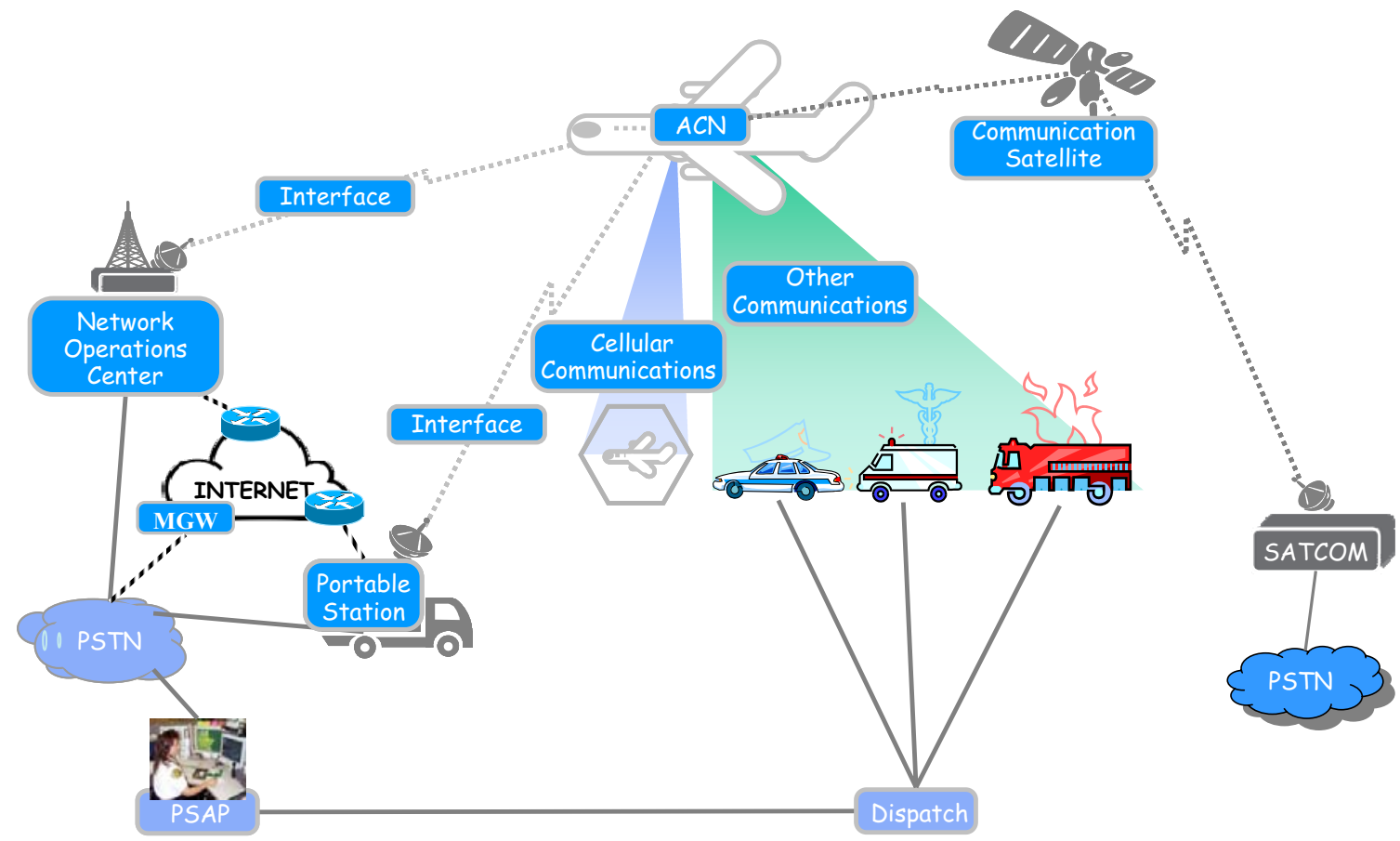

Figure 5-1: Generalized Basic Architecture

\subsubsection{Airborne Communications Node}

The ACN forms the center of the study. The purpose of this aircraft is to provide wireless communications in a catastrophic event. The ACN includes the aircraft platform and communications equipment that resides along with the aircraft. Options for aircraft platform options are covered in the Platform Options section of the report. Capabilities of aircraft communications equipment are covered in this section.

\subsubsection{Other Communications}

Other communication systems could be emergency broadcast technologies or interoperable communication systems for first responders. Broadcast technologies could include TV, FM, and AM radio broadcasts for emergency information. First responder systems could be Land Mobile Radio (LMR) 
systems or other wireless data technology. Since first responder communication technologies are diverse, disparate and evolving, there are many different options for communications technology choice.

\subsubsection{Cellular Communications}

Although there are many options for wireless technologies, cellular communications phone devices are the most prevalent technologies in the United States. Additionally, 911 services are supported on these devices. 911 and E911 are both considered critical functionality for providing emergency communications during a catastrophic event. In the U.S., Global System for Mobile (GSM) and Code Division Multiple Access (CDMA) technologies have split market share. Therefore providing GSM and CDMA services is required for 911 communications.

\subsubsection{Backhaul and Backhaul Options}

Typically, in cellular networks, the backhaul is run between the base station and the Network Operations Center (NOC) where information is exchanged between the network elements and routed to the Public-Switched Telephone Network (PSTN). Most U.S. cellular systems use T1 (E1 in Europe and Asia) links as the means to provide backhaul communications between the base stations and NOC. The $\mathrm{T} 1 \mathrm{~s}$ are typically microwave links or some form of fiber lines that carry signaling, data, and control messaging that are vital in the operation of the cellular system. A T1 contains 24 channels, either voice or data, with a total data rate of 1.544 Mbps.

In the case of the $\mathrm{ACN}$, this backhaul has three different options: direct to a terrestrial receiving station, direct portable ground receiving station, or through a satellite link. For the connections to terrestrial or portable ground stations, this could be microwave links. T1 satellite links are a very common practice and provide another viable option.

\subsection{First Responder Communications}

As explained in the Emergency Communications section, first responders do not all have the same type of radio systems. After a catastrophic event or a natural disaster, terrestrial networks should be considered severely debilitated or destroyed. Therefore, the replacement communications system could either utilize existing devices or distribute new devices after the catastrophic event. The following subsections are backup communication options for first responders after a catastrophic event.

\subsubsection{Cellular}

Cellular should also be considered for interoperable communications for first responders. Due to its low cost, wide spread availability and ubiquity, cellular communication is probably the most interoperable form of wireless communications that is available today. Additionally, the data rates provided by cellular technologies can provide much of the mission critical capabilities that are required by first responders. Cellular technologies typically have more delay in communications rather than LMR systems. This is important for mission-critical voice communications where instant communications can make a difference between life or death.

\subsubsection{Project 25 Radio Handout}

If during a catastrophic incident, an $\mathrm{ACN}$ was deployed and first responder communications were needed, P25 radios could be handed out to those needing interoperable communications. Additionally, existing P25 radios would work on the existing system with some configuration changes. This would require a cache of radios with a generic pre-configuration to be available and handed out to first 
responders after the incident. A P25 compliant system is the greatest common denominator in a variegated set of disparate first responder communication systems.

There are some disadvantages using a P25 system with phone handout. It takes time to coordinate and distribute of radios. During the first 24-48 hours communication is of paramount importance and this handout could waste the few precious hours where the $\mathrm{ACN}$ would be most effective. There could also be issues with P25 systems on aircrafts, in particular with Doppler Effect. P25 uses very narrow Frequency Division Multiple Access (FDMA) channels (12.5 kHz) [72] and, in its digital form, a Time Division Multiple Access (TDMA) scheme similar to GSM (GSM uses $200 \mathrm{kHz}$ FDMA channels) [73]. P25 Doppler limitations could be much worse than or equal to that of GSM.

\subsubsection{Audio Gateway}

Although an audio gateway provides a flexible option for first responder communications, there are also some issues with deploying this type of solution. Spare radios must be obtained from the first responders in order to integrate them into the gateway. This would require coordination with many different groups. Because the radios are added ad hoc, this means that physical connections, talk groups, and configurations must be created in order to make the system operational. Training and special operators will be required to have the system operational during ACN missions. In short, these systems are extremely flexible; however, this flexibility comes at a price of having specialized expertise and time to configure the system.

\subsection{4 $700 \mathrm{MHz}$ System}

The $700 \mathrm{MHz}$ band, which runs from $698-806 \mathrm{MHz}$, is currently occupied by television broadcasters. As a result of the Digital Television and Public Safety Act of 2005 (DTV Act), these $700 \mathrm{MHz}$ frequencies will either be auctioned for other wireless commercial services and/or made available for public safety. The DTV Act set a firm deadline of February 17, 2009 for the completion of the DTV transition with the auction of $700 \mathrm{MHz}$ band licenses scheduled for January 16, 2008. Figure 3-10, shows the planned spectrum usages of the $700 \mathrm{MHz}$ Band. [74] 


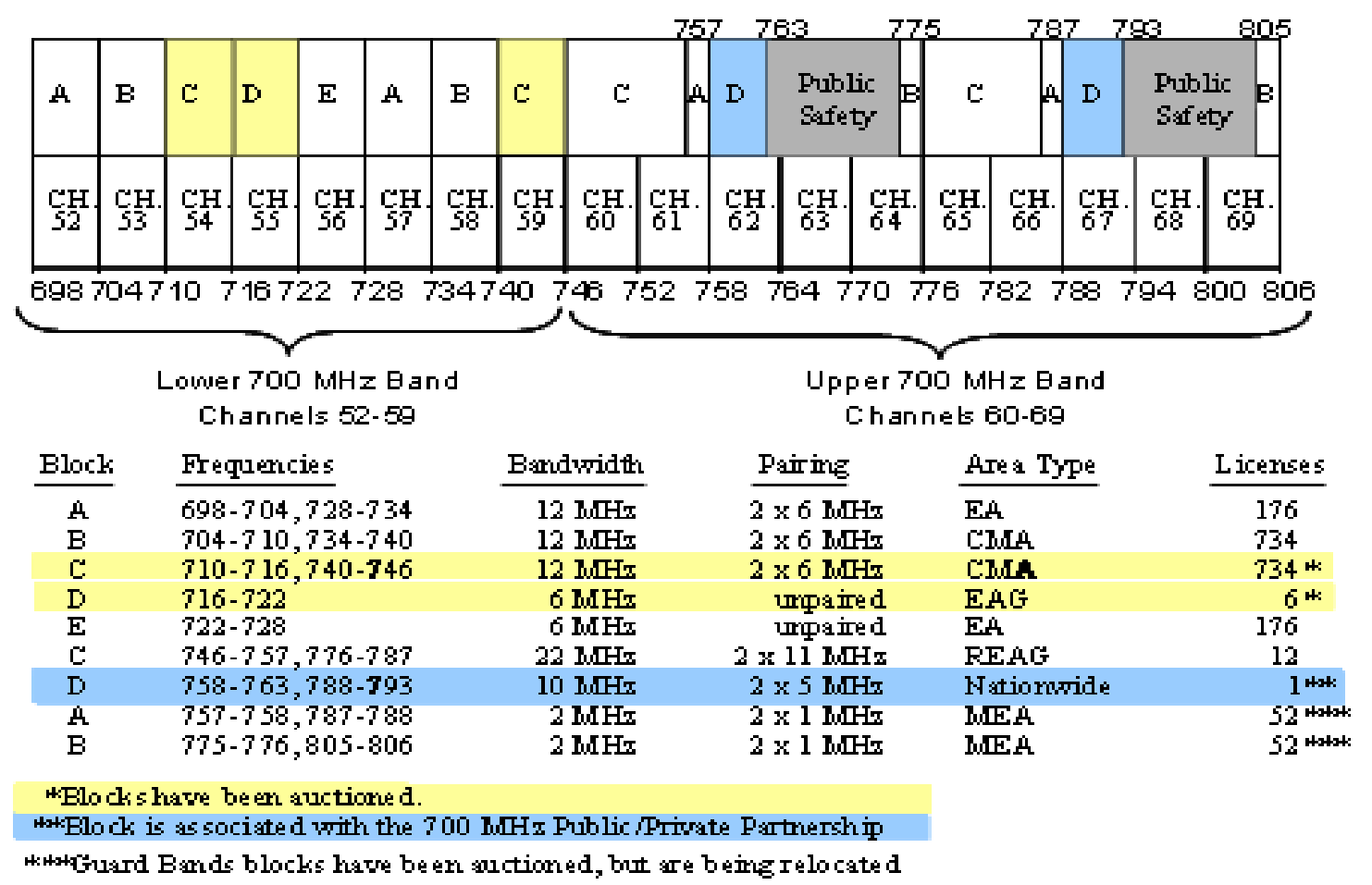

Figure 5-2: Planned Use of $700 \mathrm{MHz}$ Band

The key importance of the new auction is in the upper D blocks (shown in blue, Figure 3-10). This new licensee will have a single nation-wide license in which first responders will have priority access. This has been named a public/private partnership. This network will leverage shared ownership with the public, COTS end user devices, and new network technologies. It is most likely, that in this model first responders will have a state-of-the-art, nationwide, interoperable communications network. This new network can come online as early as February 2009. Once operational, this system would provide a good option for first responder communication on aircrafts. There are two disadvantages of using the new network. First the network has not been created. Second if the network is built, first responders may or may not migrate to this network. This might mean that a phone handout would still be required.

\subsubsection{Software Defined Radio System}

A Software Defined Radio (SDR) platform on board the ACN could enable the first responder radio system to be dynamically recreated on the aircraft. Ideally, this system would listen to transmitting frequencies, tune antennas to the appropriate frequencies, and enable recoding of existing air interface protocols so that the devices could communicate again. Additionally, it could also provide an interoperable communications bridge so that different devices can operate on the same talk groups.

One advantage of using SDR is that any existing LMR devices could be utilized providing a faster option for interoperable communication using an airborne platform. There are a couple of disadvantages of an SDR system, such as there are not any COTs equipment available, if an SDR was used on the ACN it would need to be custom built. A custom-built system could be relatively more expensive than the other solutions presented. However, leveraging the existing development of the Joint Tactical Radio System (JTRS) to include public safety frequencies and protocols could lower the costs of development. Figure 5-3 shows a simple first responder SDR architecture that could be deployed on the ACN. 


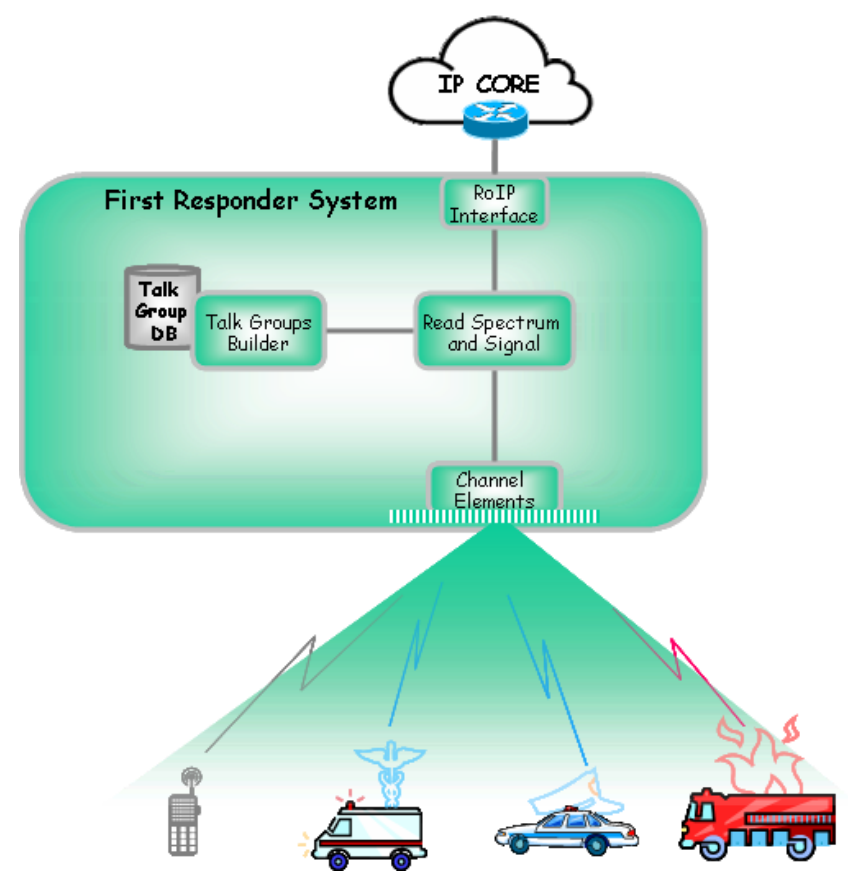

Figure 5-3: Simple SDR Architecture

\subsubsection{Summary}

Providing first responder interoperability, especially during a catastrophic event, is not a trivial problem. The problem is primarily a policy and economic problem for which there are a variety of solutions each with its trade-offs. Table 5-1 lists each potential solution and the relative trade-offs that could be deployed on an ACN.

Table 5-1: Relative Comparison of First Responder Systems for ACN

\begin{tabular}{|c|c|c|c|c|c|}
\hline & Cellular & $\begin{array}{c}\text { P25 Phone } \\
\text { Handout }\end{array}$ & Audio Gateways & 700 MHz System & SDR Platform \\
\hline Cost & Low & Medium & Low & Medium & High \\
\hline Operational Complexity & Low-Medium & Low & High & Low & Medium \\
\hline Deployment Time & Fast & Medium & Slow & Fast & Fast \\
\hline Capacity & High & High & Low & High & Medium \\
\hline Payload Weight & Medium & Medium-High & Low & Medium & High \\
\hline Maturity & $\begin{array}{c}\text { Commercial Systems } \\
\text { Operational }\end{array}$ & $\begin{array}{c}\text { Commercial Systems } \\
\text { Operational }\end{array}$ & $\begin{array}{c}\text { Commercial Systems } \\
\text { Operational }\end{array}$ & Conceptual & $\begin{array}{c}\text { Military Systems (JTRS) in } \\
\text { Development }\end{array}$ \\
\hline Comments & $\begin{array}{c}\text { Existing Terrestrial Based } \\
\text { Solutions }\end{array}$ & $\begin{array}{c}\text { Radio Caches could be } \\
\text { expensive } \\
\text { Handouts need } \\
\text { coordinated }\end{array}$ & $\begin{array}{l}\text { Requires coordination } \\
\text { and expertise to setup }\end{array}$ & Earliest Available \\
System Feb 2009 & $\begin{array}{c}\text { No Public Safety Systems } \\
\text { Available }\end{array}$ \\
\hline
\end{tabular}

\subsection{Cellular Communications Using Aircraft}

Depending on aircraft payload capabilities, configuration choices for equipment will be different. However, all components of the architecture will still be required. There are three main of configurations options for deploying an airborne cellular system. The system can be deployed as an aircraft repeater, a system on an aircraft or a Base Transceiver Station (BTS) on an aircraft. 


\subsubsection{Aircraft Repeater}

This technique combines cellular repeater equipment and aircraft to expand coverage of the network. This system uses existing network signals and repeats them in order to expand the coverage of the network.. The objective of this architecture is to translate and repeat the mobile signals back and forth to the BTS. Figure 5-4, shows a block diagram that illustrates how this architecture works. A beam forming antenna could be used to send and receive signals to the service area. This is done to minimize outside interference and improve the gain on the existing networks. A Low Noise Amplifier (LNA) and Band Pass Filter (BPF) are used to improve the signal. A BTS simulator is then used to replicate the air interface protocol to the mobile. The frequency and signal is then transformed using a Mux/DeMux. A second beam forming antenna is used to send the signal back to the terrestrial infrastructure. [76][77] This can be done by using either a cellular link protocol or other radio frequency to a converter station to send back to the Base Station Controller (BSC) via an Backhaul link. [78] As stated before, this is a generic diagram to explain the repeater architecture concept.

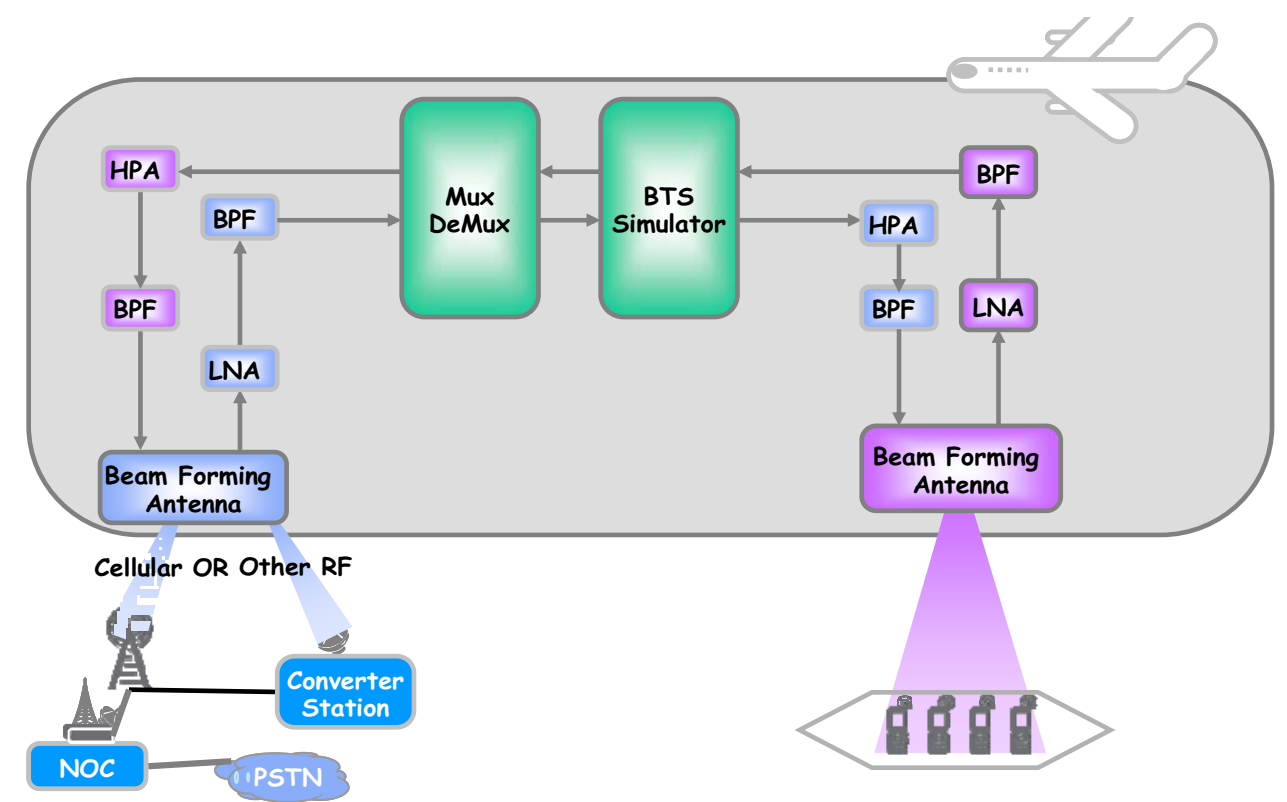

Figure 5-4: BTS Repeater Architecture

\subsubsection{Commercial Off the Shelf Repeaters}

There are several COTS repeaters available that perform these functions and many vendors who make GSM and CDMA repeaters. Figure 5-5 shows two different Guanri Telecom-Tech (Shenzhen) Co., Ltd, repeaters that are available COTS [79]. It is very likely this could easily be strapped onto a small aircraft to provide quick extended coverage. The repeater architecture could also be used for LMR radio technologies. Ideally, these systems should be augmented with antennas, which are able to steer the antenna beam and adapt coverage to a moving aircraft. 

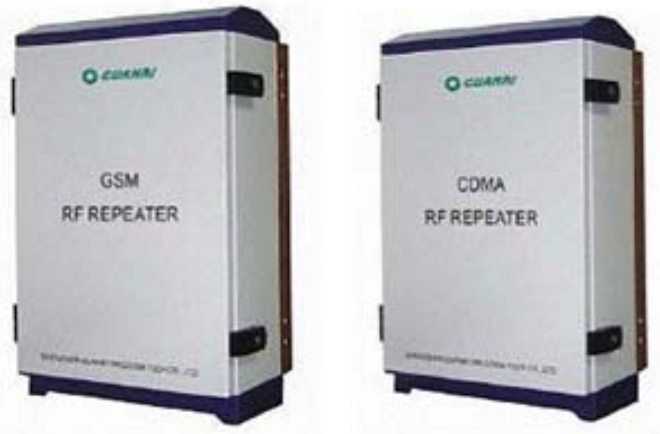

Figure 5-5: Cellular BTS Repeaters [79]

\subsubsection{Repeater Concept of Operation}

The following example illustrates how a repeater solution would fit into an operational scenario. Once the aircraft is dispatched, an outage report is sent from the NOC. The outage report defines Out of Service (OOS) areas and neighboring cell frequencies that can be repeated. The aircraft repeater uses this information and repeats the signals to the outage areas. In Figure 5-6, the NOC and some of the BTSs are operational these BTS signals are repeated in order to provide coverage to the outage areas.

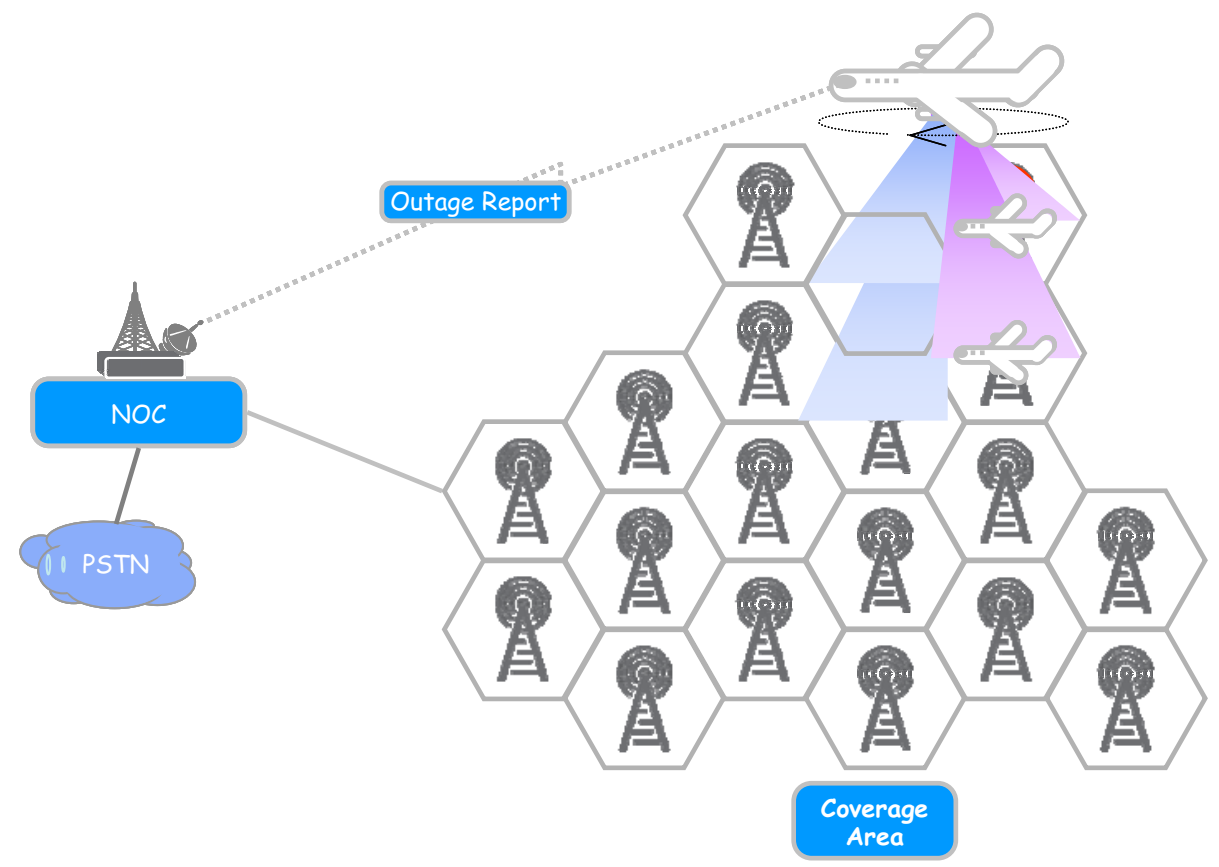

Figure 5-6: BTS Repeater Scenario

\subsubsection{System on an Aircraft}

A system on aircraft is the concept where a completely independent communications system is provided utilizing an aircraft. A cellular system on an aircraft requires all the network elements of cellular system. Figure 5-7 shows the Mobile Switching Center (MSC), BSC, and BTS elements of a GSM and CDMA system and three different backhaul to the PSTN. The backhaul signals in this diagram consist of Signaling System 7 (SS7) interface that must be provided to the PSTN for outside phones to be reached. This basic diagram shows a possible configuration that could be placed on an aircraft. However, there are 
many different possibilities depending on if normal telecom equipment is used or if a system in a box is used on the aircraft.

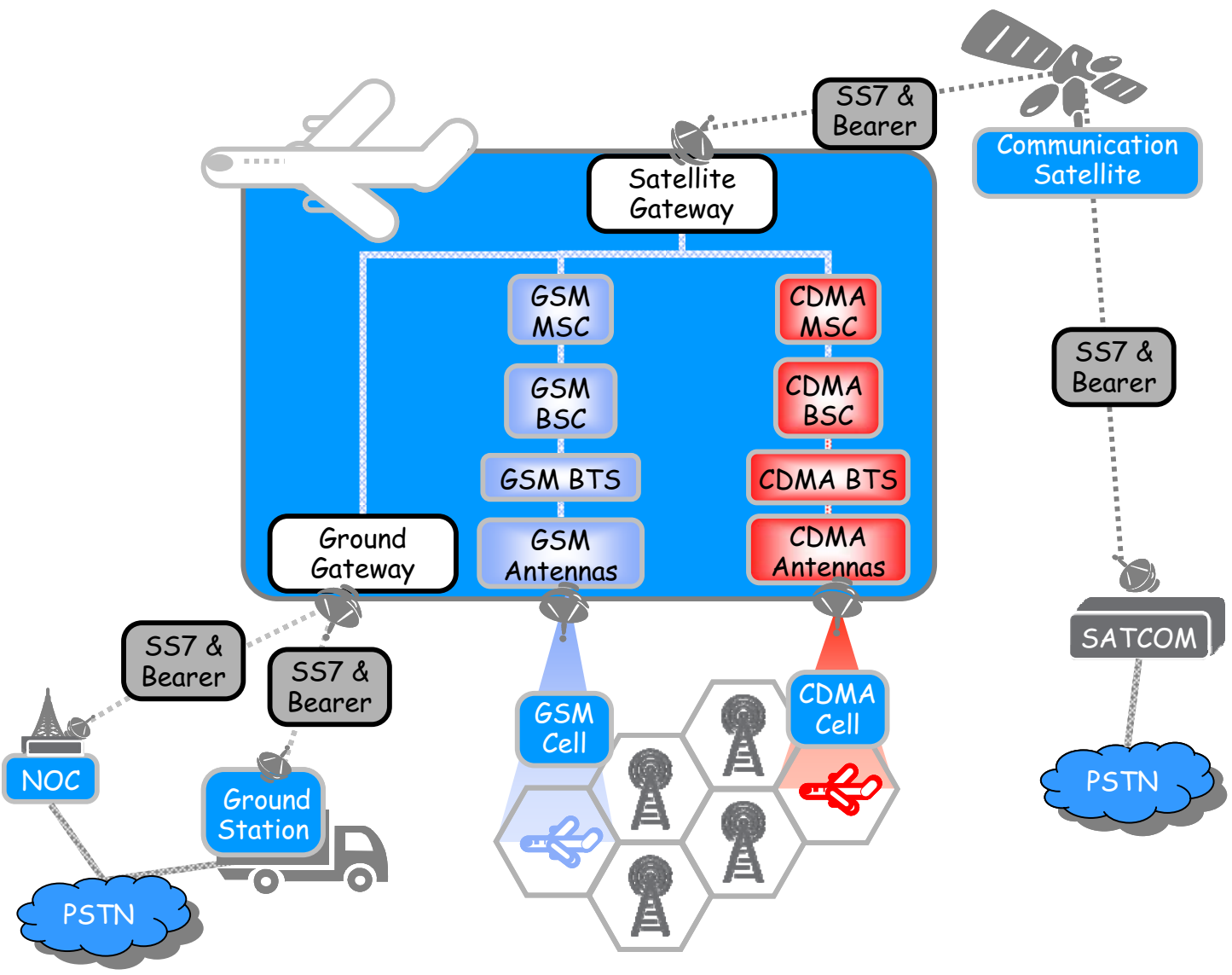

Figure 5-7: System on an Aircraft

Using normal telecom equipment, the telecom equipment deployed by commercial carriers, could mean many stand-alone racks for each network element. A minimum configuration could be four to six racks each weighing at least $\sim 500 \mathrm{lbs}$ each. Using such a system could provide a higher capacity than a system in a box. However, these systems are not designed for minimum weight. These types of systems should be flown on a large aircraft with a large amount of payload capacity ( $>10$ tons). Example vendors of commercial cellular systems include Motorola, Nokia, Siemens, Nortel, Huawei, and Nortel.

Another option for providing this technique is to use a system in a box. There are vendors that manufacture entire cellular systems in a single box. These platforms provide good options for strap-on solutions. Figure 5-8 shows two examples of systems in box. 


\section{Qualcomm QDBS}

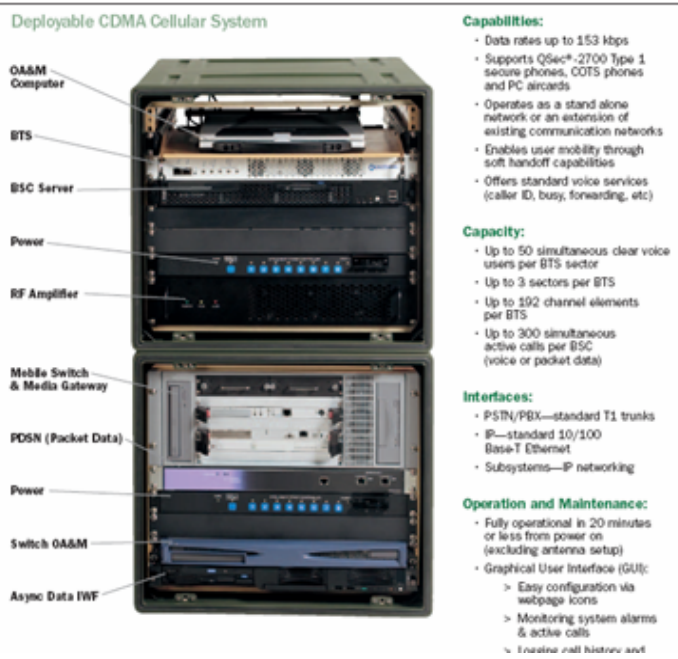

TLCS IGN
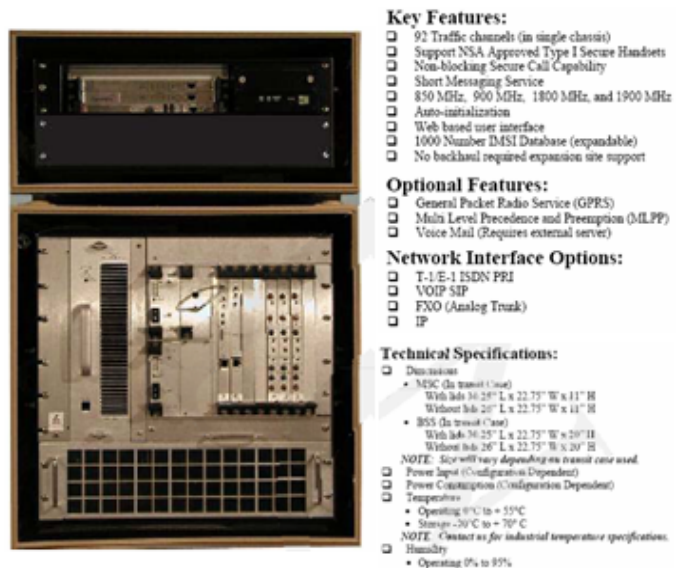

Figure 5-8: Cellular Systems in Box [80][81]

The Qualcomm Deployable Base Station (QDBS) is a popular CDMA platform and has been used on at least two different airborne communications experiments. One experiment was conducted with Northrop Grumman using the NASA B-57 flying at $\sim 60 \mathrm{k} \mathrm{ft} \mathrm{[82].} \mathrm{The} \mathrm{second} \mathrm{experiment} \mathrm{was} \mathrm{done} \mathrm{by}$ AeroFon that used a Cessna $421 \mathrm{C}$ at 3k, 7k and 17.5k ft. [83] Additionally, NORTHCOM, Federal Emergency Management Agency (FEMA), and Air National Guard use this platform in their emergency communications architectures. The second example shown is the Top Line Communication Systems (TLCS) Integrated GSM Node (IGN) a GSM platform, with very similar functionality and size as the QDBS.

\subsubsection{BTS on Aircraft}

Another possible configuration for providing cellular communications on an aircraft is to provide the $A_{\text {bis }}$ link to a BTS on the aircraft using one of the three backhaul options with terrestrial BSC and MSC. This configuration is essentially a "flying BTS." In fact, it is not unusual for operators to deploy base sites in remote locations or on cruise ships using a satellite link for the $A_{\text {bis }}$ link. Although, some special configuration is required on the $\mathrm{A}_{\text {bis }}$ link to compensate for the delay using a satellite link. Figure 5-9 shows an architectural diagram of this configuration. 


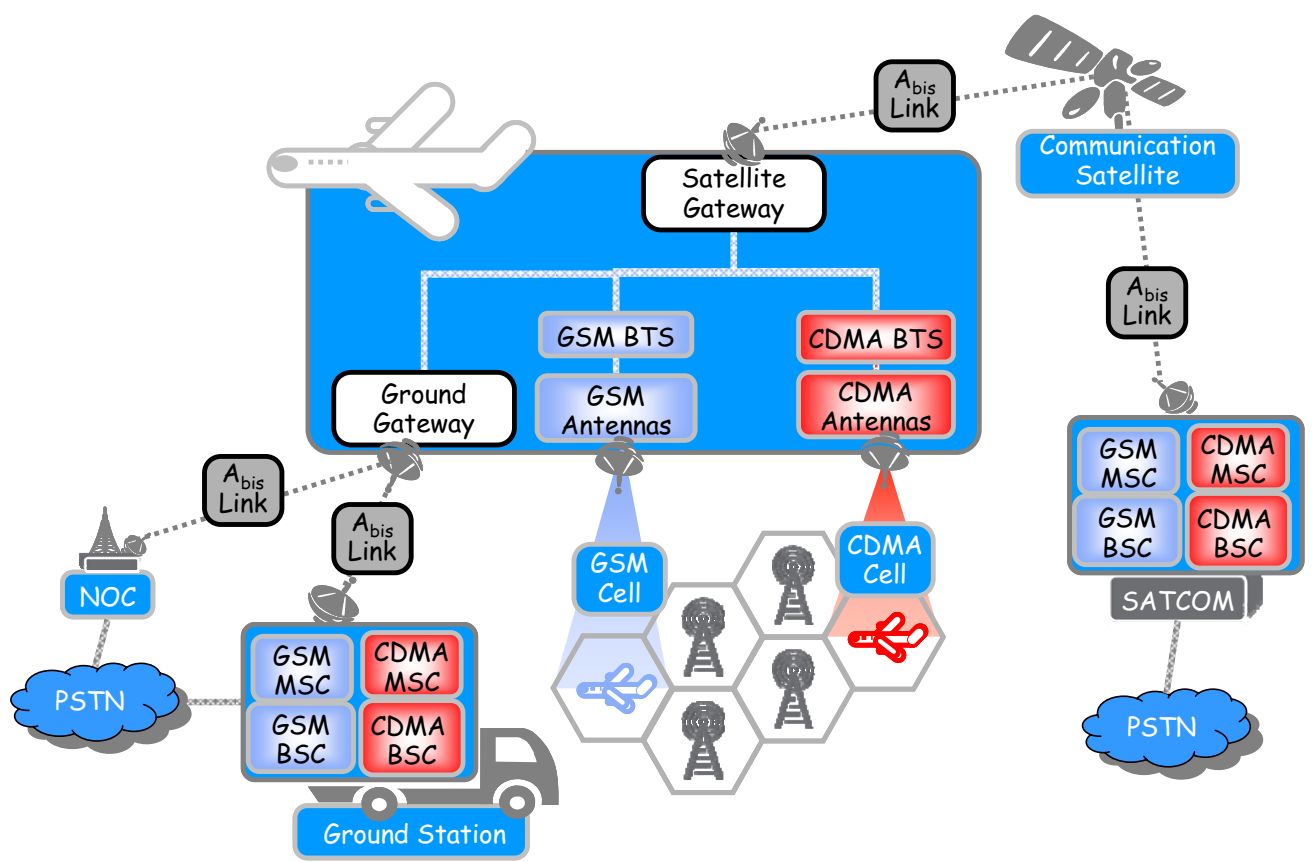

Figure 5-9: BTS on Aircraft Architecture

\subsubsection{Summary}

Cellular communications on an aircraft is not a novel concept. There are many vendors who offer solutions to provide cellular communications using airborne vehicles and have demonstrated its viability. Each solution has its own tradeoffs. Table 5-1 shows each potential solution and its relative tradeoffs agnostic of an aircraft platform.

Table 5-2: Airborne Cellular System Options

\begin{tabular}{|c|c|c|c|c|}
\hline & Aircraft Repeater & $\begin{array}{c}\text { System on Aircraft } \\
\text { w/carrier grade } \\
\text { equipment }\end{array}$ & $\begin{array}{c}\text { System on } \\
\text { Aircraft } \\
\text { w/system in a Box }\end{array}$ & BTS on Aircraft \\
\hline Cost & Low & Medium & Low & Medium \\
\hline $\begin{array}{c}\text { Operational Complexity } \\
\text { Aircraft }\end{array}$ & Low & High & Low & Medium \\
\hline $\begin{array}{l}\text { Operational Complexity } \\
\text { Ground Station }\end{array}$ & Low & Low & Low & High \\
\hline Deployment Time & Fast & Fast & Fast & Fast \\
\hline Call Capacity & Low & High & Low & High \\
\hline Coverage & Low & High & Low-Medium & Medium \\
\hline Payload Weight & Low & High & Low & Medium \\
\hline Maturity & COTS Repeaters Avaiable & Not been done & \begin{tabular}{|c|} 
Commercial Systems \\
Demonstrated on Fixed \\
Wing Aircraft
\end{tabular} & $\begin{array}{l}\text { Systems Demonstrated } \\
\text { on Tethered Aerostats }\end{array}$ \\
\hline Comments & $\begin{array}{l}\text { Existing system needs to } \\
\text { be operational }\end{array}$ & $\begin{array}{l}\text { SS7 links over Air } \\
\text { Direct Tie into PSTN }\end{array}$ & $\begin{array}{c}\text { SS7 links over Air } \\
\text { Direct Tie into PSTN }\end{array}$ & $\begin{array}{l}\text { Abis Links over Air } \\
\text { BSC, MSC needed in } \\
\text { Ground Stations }\end{array}$ \\
\hline
\end{tabular}




\subsection{Additional Emergency Communications Capabilities}

There are additional capabilities that the ACN could provide in support of a disaster. This equipment could be added to the overall architecture. The following list identifies those additional capabilities that would be beneficial on an aircraft.

\subsubsection{FM Transmitter}

An FM transmitter is a simple addition that would increase the ability to disseminate information. The ACN could receive information using one of the three backhaul options and deliver the information when in flight using an FM broadcast. Residents could use FM radios in the event of an emergency to get important information such as collection points, supply pick-up points, and shelters. Since most states already have information networks that include FM, much of the same alerting infrastructure could be used.

\subsubsection{Wireless E911 Support}

Wireless E911 has become a vital function for first responders. The ability to locate those in distress is an extremely valuable capability, especially when locating those who need rescued. For this reason, wireless E911 functionality should be included in the cellular system onboard the aircraft. This would enable location of individuals in distress and aid rescue efforts.

\subsubsection{Wireless Priority Service}

Wireless Priority Service (WPS) is used to give priority use of public wireless networks to government officers or even first responders in times of high network congestion. This could be used so that first responders can access the network while simultaneously allowing many users onto the network. This capability was recommended from the network operators.

\subsubsection{Emergency Cellular Broadcast}

Emergency cellular broadcast is also an important function that uses the cellular air interface to broadcast emergency messages to mobile phones similar to text messaging. Cellular operators in the U.S. are currently implementing this capability on their networks. This function would allow the cellular network to send broadcast messages to all cellular phone users in the affected area. Messages could contain information like escape routes, food and water points, and hazards to be avoided. Using cell broadcast everyone with a cellular phone can receive vital information.

\subsubsection{Satellite Phone Relay}

Satellite phones are susceptible to poor quality due to cloud cover. To overcome this problem the ACN could be used as a satellite phone relay by maintaining a flight path over clouds.

\subsection{Recommended Architecture}

For an emergency ACN platform to be effective, its capabilities must be integrated into a complete emergency communications architecture. To communicate with existing devices, the cellular communications systems on the ACN must be a representative of the existing cellular ecosystems. Communication systems on the ACN should be interoperable using gateways and an IP core to allow communications between all networks. Additionally, E911 capabilities should also be leveraged to assist in disaster relief efforts. Backhaul is required to transmit voice and data information into and out of the ACN. E911 call data should be sent to the remote public safety answering point (PSAP) and the 
Emergency Operations Center (EOC). Figure 5-10, shows a diagram of this architecture. A more detailed explanation of this figure is given in the following subsections.

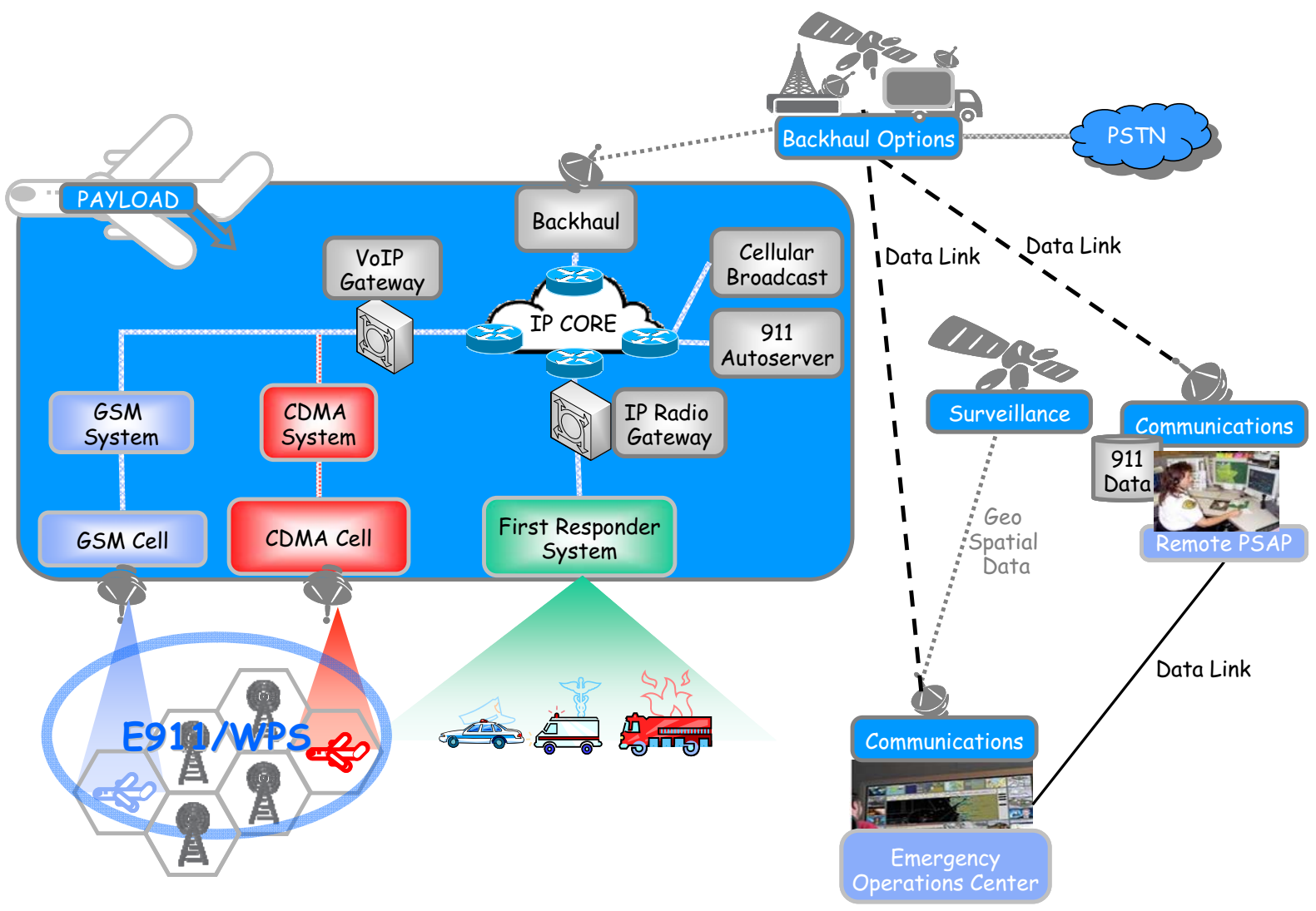

Figure 5-10: Recommended Architecture

\subsubsection{Cellular System}

Since GSM and CDMA cellular technologies make up almost equal market share in the U.S. and mobile devices will rely on these technologies for the next 5-10 years, both a GSM and CDMA system need to be supported on the aircraft. These systems form the basis for supporting E911 functionality, which will support relief efforts and additional communications for first responders. The system should be configured with special access rules to allow for all phones to make 911 calls and allow exclusive access for first responder devices. Additionally, cell broadcast capability should also be supported.

Exclusive access could be provided by either an open access cellular network with WPS or a specially configured Home Location Register (HLR) for the cellular network. By using an open cellular network with WPS, any user could make any call, including 911. First responders would use WPS for priority access. This is how current public cellular networks behave. Alternatively, the HLR could be configured to allow only specific mobile devices - first responder devices-onto the network. This network, using a specially configured HLR, must only allow 911 calls. This is also how currently cellular networks behave. In both cases, this would require that the cellular network be connected to the PSTN to enable the WPS functionality and roaming of first responder's mobiles onto the network. Using an open network with WPS, first responders will likely experience more traffic and could hinder their ability to communicate. Using network with specifically configured HLR would allow first responders exclusive access, while other users could make only 911 calls. 


\subsubsection{Radio Gateways and IP Core}

The cellular Voice over Internet Protocol (IP) gateway, Radio IP gateway, and IP core form the center point for interoperable communication. In order to provide an interoperable interface for all communications systems, gateways and an IP core are necessary. This is to enable these networks to intercommunicate. This technique has been used by many interoperability vendors. [84][85] This type of core network is COTS available.

\subsubsection{Wireless E911 Methods on Aircraft}

In order to facilitate search and rescue operations, the ACN should provide E911 location capabilities. E911 mobile location methods are different for CDMA and GSM. CDMA typically uses assisted-GPS while GSM uses triangulation techniques. Because CDMA mobiles use GPS it is relatively easy to locate them. Unfortunately, triangulation techniques used by GSM require two or three BTSs to accurately locate mobiles.

One potential method for GSM mobile location is creating a system that combines on-board aircraft navigation with position determining equipment (PDE), which can calculate the angle of arrival (AOA) and the time delay of arrival (TDOA). This system could determine the location of the mobile device with respect to the aircraft. Figure 5-11, shows a basic functional diagram. Position determining equipment is available COTS, but it would require specific modifications to enable location finding capabilities with aircraft navigation equipment.

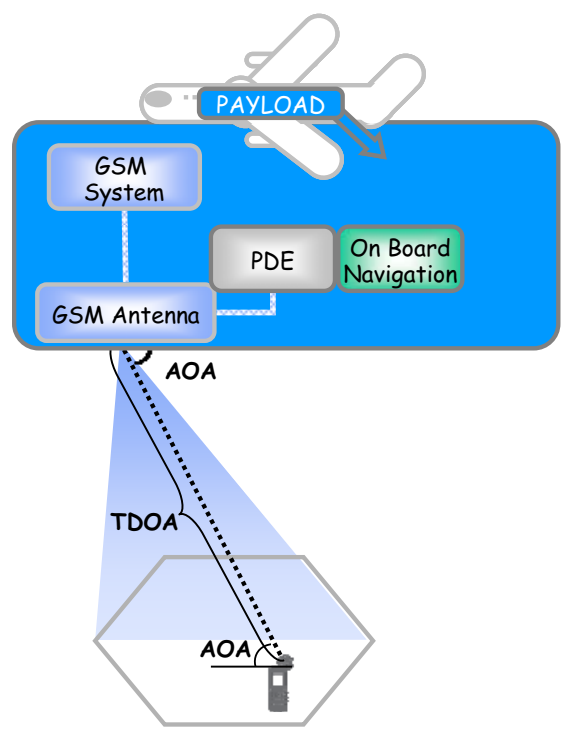

Figure 5-11: AOA, TDOA w/Navigation

Another option for locating mobiles was tested by Japan's National Institute of Information and Communications Technology (NiCT). A pictorial representation of the experiment is shown in Figure $5-12$. 


\section{High-Resolution Radio Localization Experiment using 'Global Observer Prototype'}

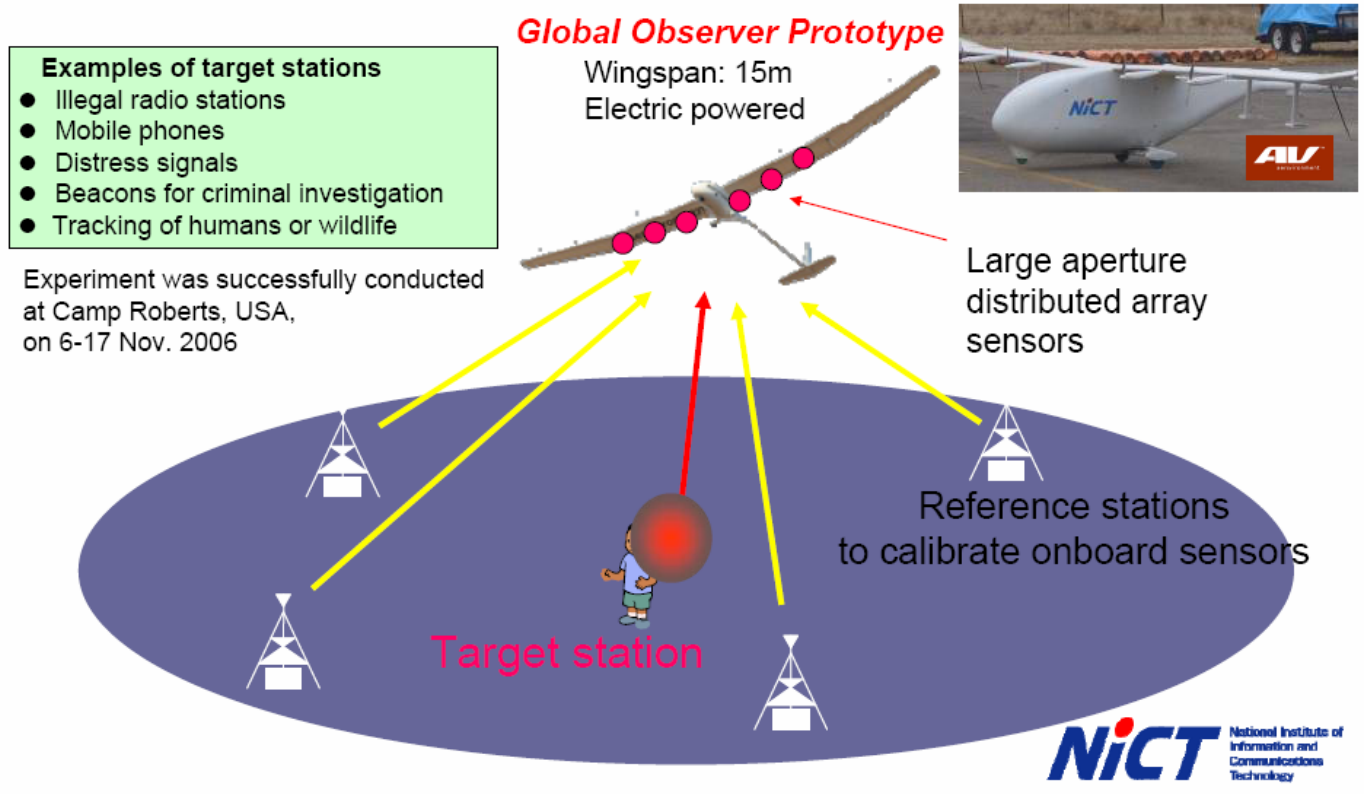

Figure 5-12: NiCT Experiment [88]

The test objective was to use reference stations (BTS), whose locations were known, to calibrate the sensors on the aircraft. These sensors then took AOA measurements from the target station, which was used to calculate its location relative to the reference stations. This was done with the AeroVironment's global observer prototype flying 1,000 ft above ground. Using this technique, the NiCT was able to accurately calculate the location of the target stations within $5 \mathrm{~m}$. In a disaster situation, this technique could be used to find distress callers or anyone who carries a cell phone.

\subsubsection{Auto Server, Remote PSAP and Emergency Operations Center}

Due to the possibility of overwhelming 911 call volumes, there should be a mechanism for answering select 911 calls. This would require a 911 server that could provide automatic 911 answering capabilities or forward the calls to a remote PSAP that could answer all calls going through the ACN. The server then would track the location of the call and reply with a message to the user. This location information could then be sent to a PSAP operator who resides in a remote PSAP or other PSAP system. Regardless, the PSAP views the calls on computer-aided dispatch and is able to call those 911 callers back requesting information.

Additionally, the location data can also be sent through the network to a command and control map at the EOC. Using E911 location data and real-time voice feed from 911 callers will provide accurate situation awareness. This information would be used by commanders and civil officers to improve situational awareness. This information would be especially useful if overlaid on real-time geo-spatial imagery. As an example, when a burst of 911 calls show up as red dots on a map, commanders could "zoom in" to see what the disaster is then dispatch forces appropriately. 


\subsubsection{Backhaul Recommendations}

There are two ways to implement a backhaul for the ACN: via satellite connections from the ACN to ground stations and a high bandwidth line-of-sight link. Both of these methods are proven to be reliable backhaul links for ground-based systems, but when used for an application such as the ACN, the complexities grow substantially.

Satellite backhaul is an effective way to provide the reach-back capability that may be required during emergency operations. One of the key benefits of satellite backhaul is that it can be tailored to the bandwidth needed by adding or subtracting channels. Another benefit is that it can be received by many entities to extract the information needed from the various reporting agencies. For example, once all of the information is transmitted off of the aircraft, the PSAP can tune to the satellite channel and receive the data they need (usually voice), the PSTN can receive the data (mobile call data) it needs and interested parties can receive video and voice if required. Figure 5-13 shows how this architecture would work.

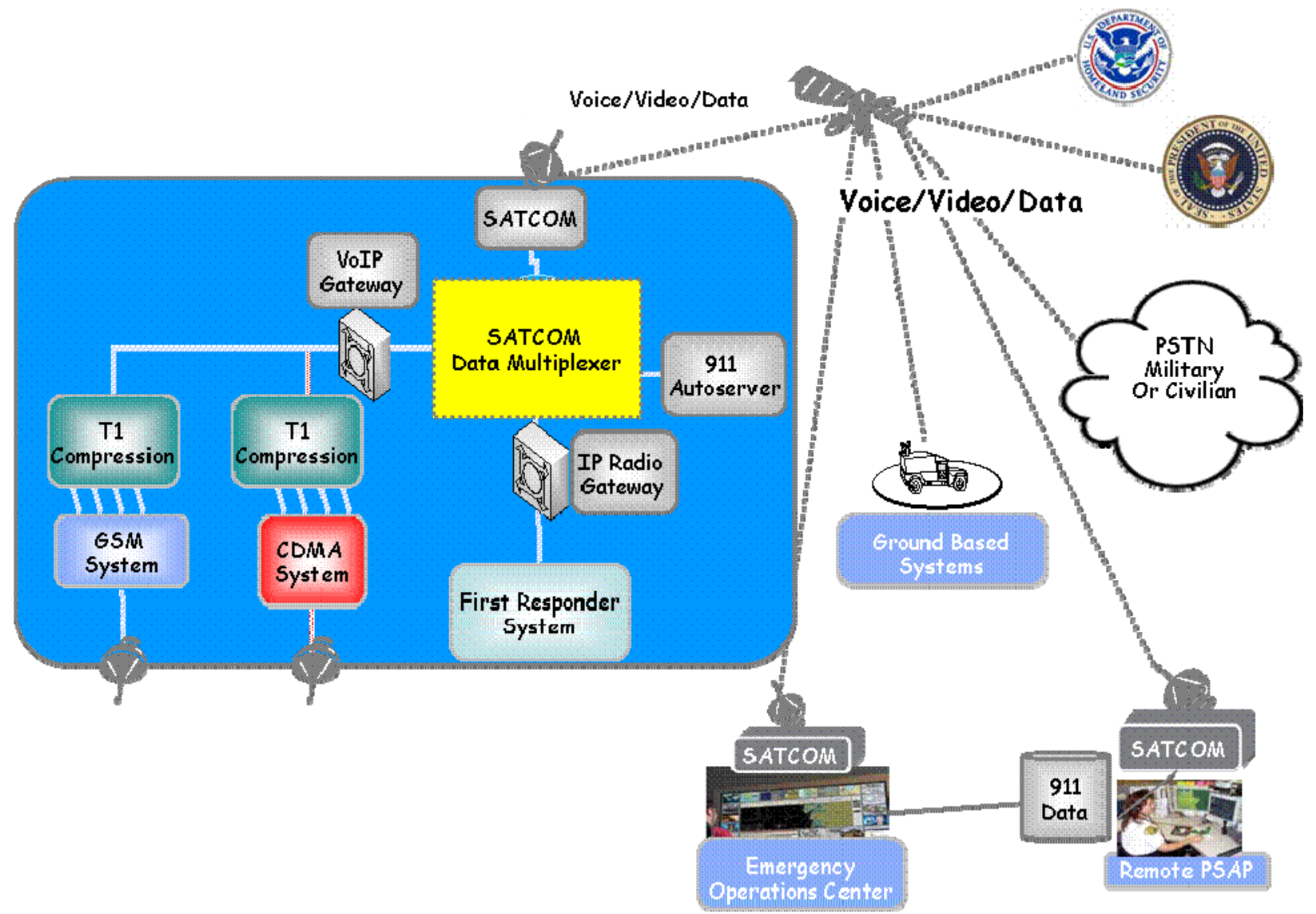

Figure 5-13: Satellite Backhaul Architecture

The key drawback for satellite backhaul is the round trip delay. The typical propagation delay for satellite systems is approximately $250 \mathrm{~ms}$. This delay may degrade voice quality between those that are not located in the area of responsibility and first responders on the ground. Messaging delays may inhibit connections between the cellular networks and PSTN due to message timeouts. This drawback may be overcome with some simple changes to software timers and other parameters.

The second method to establish reach-back connections is through a high frequency line-of-sight link that would have similar properties to a microwave link, the difference being the available bandwidth. A direct line of sight link would connect to a ground station that is capable of routing the information to 
the PSAPs, PSTNs and key stakeholders, enabling communications to be connected from the area of interest to the outside world.

The main drawback to this technique is maintaining a line of sight link while the aircraft is in motion. At VHF/UHF frequencies, this may not be a difficult task, but as the frequency and bandwidth increase, the link becomes extremely sensitive to movement. If the line of sight link were to be lost, the likelihood of dropped calls would be extremely high.

\subsection{Conclusions}

The conclusions based on different architectures, components and techniques used to provide critical communications from and ACN discussed in this section are as follows:

- ACN first responder communications systems have a few options and are problematic at best.

- ACN cellular communications system should support both GSM and CDMA technologies so that it provides equitable access for all users.

- ACN cellular communications system should support 911 calls for all phones

- ACN cellular communications system should support E911 location finding capabilities

- ACN cellular communications system should support WPS

- ACN cellular communications system should support exclusive network access to specific phones

- ACN cellular communications system support connectivity to a PSAP

- ACN cellular communications system should support connectivity to an EOC

- ACN cellular communications system should support connectivity to the PSTN to support priority access and also out-of-network calling

- PSAP and EOC should support interconnectivity and exchange of E911 location data and real time call feeds

- EOC should combine E911 location data with real time satellite imagery.

- ACN should support a capability to automatically answer 911 calls and control 911 call congestion

- ACN should use satellite channels for backhaul transmissions 


\section{REFERENCES}

[72] R. I. Desourdis, Jr., D. R. Smith, W. D. Speights, R. J. Dewey, J. R. DiSalvo, Emerging Public Safety Wireless Communication Systems, Artech House Publishers, ISBN 0-89006-575-6, 2001.

[73] Michel Mouly and Marie-Bernadette Pautet, The GSM System for Mobile Communications, Cell \& Sys, ISBN 2-9507190-0-7, 1992.

[74] FCC, "FCC Revises $700 \mathrm{MHz}$ Rules to Advance Interoperable Public Safety Communications and Promote Wireless Broadband Deployment," July 31, 2007, http://hraunfoss.fcc.gov/edocs_public/attachmatch/DOC-275669A1.doc

[75] SDR Forum, "Software Defined Radio Technology for Public Safety,” April 14, 2006, SDRF-06A-0001-V0.00, www.sdrforum.org

[76] Djuknic, G. M., Freidenfelds, J., and Okunev, Y., "Establishing Wireless Communications Services via High Altitude Aeronautical Platforms: A Concept Whose Time Has Come?” IEEE Communications Magazine, September 1997, pp. 128-135.

[77] Masayuki Oodo, Hiroyuki Tsuji, Ryu Miura, Member, IEEE, Masaaki Maruyama, Mikio Suzuki, Yuichiro Nishi, and Hisashi Sasamoto, "Experiments on IMT-2000 Using Unmanned Solar Powered Aircraft at an Altitude of 20 km," IEEE Transactions on Vehicular Technology, Vol. 54, No. 4, July 2005.

[78] D. M. McCraw, T. P. Emmons Jr., J. H. Gross, J. F. Berke, "Geolocation Techniques for an Airborne Cellular System," USPTO, Patent 6,795,699, September 21, 2004, http:/www.google.com/patents?id=tYASAAAAEBAJ\&dq=US+Patent+6,795,699

[79] Guanri Telecom-Tech (Shenzhen ) Co., Ltd., GSM and CDMA Repeaters, http://guanri.en.ecplaza.net/

[80] QUALCOMM Deployable Base Station, Qualcomm, http://www.qualcomm.com/qgov/products/qdbs.html

[81] Top-Level Communications Solutions, Integrated GSM Network (IGN), http://www.tlcconsulting.com/ign.html

[82] BACN CDMA Subsystem Test Results, June 02, 2006, Northrop Grumman.

[83] AeroFon, “Airborne CDMA Cellular System Demonstration Report” AeroFon Corp. \& QUALCOMM Government Systems Group, May 29, 2003, http://www.aerofon.com/DemoSummary.htm

[84] Henry S. Kenyon, “Secure Cellular System Serves Warriors and Rescuers,” Signal, January 2007.

[85] Teresa Schofield, "Dallas Launches City-Wide Interoperability Network," CoCo Communications Press Release, November 15, 2006,

[86] D. M. McCraw, C. C. Matthews, M. M. Madine, C. L. Fullerton, J. D. St. Peter, "Transportable Infrastructure For Airborne Cellular System," USPTO, Patent 6,804,515, October 12, 2004, http://www.google.com/patents?id=SesRAAAAEBAJ\&dq=US+Patent $+6,804,515$ 
[87] National Public Safety Telecommunications Council, "Interoperable Communications Technical Assistance Program (ICTAP), Audio Gateway Handbook," March 2006, ICTAP-OG\&T-HNDBK002-R0 http://www.npstc.org/documents/

[88] Hiroyuki Tsuji, National Institute of Information and Communications Technology (NICT) Japan, "Recent Developments for HAPS Applications in Japan," Whales Conference, March 2007, Palmdale, California. 


\section{ACRONYMS}

\begin{tabular}{|c|c|}
\hline $\mathrm{ACN}$ & Airborne Communications Node \\
\hline $\mathrm{AOA}$ & Angle Of Arrival \\
\hline BPF & Band Pass Filter \\
\hline $\mathrm{BSC}$ & Base Station Controller \\
\hline BTS & Base Transceiver Station \\
\hline CDMA & Code Division Multiple Access \\
\hline COTS & Commercial off the Shelf \\
\hline DTV & Digital TV Act of 2005 \\
\hline EOC & Emergency Operations Center \\
\hline FDMA & Frequency Division Multiple Access \\
\hline FEMA & Federal Emergency Management Agency \\
\hline GSM & Global System for Mobile \\
\hline HLR & Home Location Register \\
\hline ICTAP & Interoperable Communications Technical Assistance Program \\
\hline IGN & Integrated GSM Network \\
\hline IP & Internet Protocol \\
\hline JTRS & Joint Tactical Radio System \\
\hline LMR & Land Mobile Radio \\
\hline LNA & Low Noise Amplifier \\
\hline MSC & Mobile Switching Center \\
\hline $\mathrm{NiCT}$ & National Institute of Information and Communications Technology \\
\hline NOC & Network Operations Center \\
\hline OOS & Out of Service \\
\hline PDE & Position Determining Equipment \\
\hline PSAP & Public Safety Answering Point \\
\hline PSTN & Public-Switched Telephone Network \\
\hline QDBS & Qualcomm Deployable Base Station \\
\hline SDR & Software Defined Radio \\
\hline SS7 & Signaling System 7 \\
\hline TDMA & Time Division Multiple Access \\
\hline TDOA & Time Delay of Arrival \\
\hline TLCS IGN & Top Level Communications Solution Integrated GSM Node \\
\hline UHF & Ultra High Frequency \\
\hline
\end{tabular}


Very High Frequency

VLR

Visitor Location Register

WPS

Wireless Priority Service 


\section{Antennas}

Modern communications systems require extremely sensitive and complex antennas. Many industry experts have begun calling antennas "systems" due to the engineering that is involved developing them for increased sensitivity and capacity while decreasing the antenna footprint and cost. In the beginning, antennas needed to be frequency matched, polarized correctly, and phase matched. Additionally, the receiver or transmitter systems were responsible for increasing sensitivity and transmitting power (to increase coverage), respectively. In current antenna systems, in addition to frequency matching, polarization, and phase matching, the antennas are designed with multiple elements, algorithms to increase the signal-to-noise ratio (SNR), and algorithms to dynamically form beams or switch elements. In addition to the previously mentioned capabilities, these systems must then be miniaturized and inexpensive. Antennas have evolved from a piece of equipment used for many applications, to being an entire system that requires matching to a specific application. For example, a single element blade antenna can be used for nearly any type of communication system, either line of site (LOS) radios or cellular systems. However, a Multiple Input Multiple Output (MIMO) antenna would be impractical if used with the typical LOS systems, even at the same frequencies, due to the complexity of its processing algorithms and specialized development for Orthogonal Frequency Division Multiplexing (OFDM) technology.

Antenna system classification can be broken down into two basic parts: conventional and specialized. Conventional antennas are single or dual element antennas that act only as an electrical interface to transmit or receive radio waves and have no beam steering or processing capability. Conventional antennas are common to applications for which they are intended, whether they are cellular or land-mobile radio-based. Some examples of conventional antennas are Omni blade antennas, most commercial off-the-shelf (COTS) antennas, and antennas without associated Digital Signal Processors (DSP). Conventional antenna systems usually provide uniform transmitting power dispersion and coverage areas. These types of antenna systems are relatively inexpensive, which is why most cellular systems today use various types of typical antennas. Specialized antennas are any antenna type that is specific to a certain application. They usually contain multiple elements that may transmit and receive simultaneously. These include DSPs for adaptive technologies, beam forming capabilities, and more exotic signal canceling capabilities. Some of these antennas are finding their way into commercial applications as the cellular technologies evolve. Both of these antenna types are discussed in the following subsections.

\subsection{Cellular Antenna Overview}

When selecting the antenna system for cellular systems, whether the system is Global System for Mobile (GSM) communications or Code Division Multiple Access (CDMA), three factors need to be taken into consideration: sensitivity, coverage/capacity, and hardware robustness. First, and most important, is the sensitivity of the antenna. Certain types of antennas (e.g., adaptive antennas) can show a reasonable sensitivity gain over more common single element or passive antennas. Usually this difference can be more than $10 \mathrm{dBi}$. The increase in sensitivity translates to an increase in coverage area, as well as capacity gain. The antenna sensitivity can also be correlated to the mobile transmit power. In other words, the more sensitive the antenna, the less transmitting power is required by the mobile station, which reduces the base station-received noise floor and lengthens the mobile station battery life.

The second factor that should be considered is the antenna beam width. The beam width will affect the coverage area, as well as the capacity of the given sector or cell. Generally, the tighter the beam width, the higher the concentration of transmitting power in a given area. This high concentration of

transmitting power extends the reach of the coverage area. A second benefit of a narrow beam width is increased capacity, which is the product of the decrease of the receiver noise floor due to the lack of 
external interference. For instance, compare an Omni antenna with a $30^{\circ}$ beam width antenna. The Omni antenna will receive signals from a $360^{\circ}$ area, increasing the noise that the system will receive, and raising the receiver noise floor. Conversely, the $30^{\circ}$ beam width antenna will only receive signals within the beam width, thus reducing the receiver noise floor. In cellular systems, a narrow beam width, coupled with increased output power and received sensitivity, increases the probability that a cellular access attempt will be successful in a heavy-loaded area.

Third, is the robustness of the antenna hardware. For this application, the typical $120^{\circ}$ sector commercial antenna will not be able to sustain the stress required, nor is it practical to mount this type of antenna on an aircraft. In fact, the typical rated wind speed for a commercial antenna is $150 \mathrm{mph}$. [90] Additionally, temperature differences and pressure differences experienced during flight may have an adverse impact on the performance of the antenna. For these reasons, alternative antennas must be explored. These antennas include single element blade antennas, single and multiple element directional antennas, and advanced adaptive antennas.

\subsection{Conventional Antenna Configurations}

Although there are many types of conventional antenna types, this section addresses the two key configurations that the Airborne Communications Node (ACN) can deploy. These configurations, Omni and Directional, are common throughout the cellular industry and have a proven record in reliability and consistency. These antennas have crossover capability into first responder networks as well, as long as they are frequency matched to the first responder network. For the purposes of this section, all of the conventional antenna configurations have fewer than two elements.

\subsubsection{Omni Antenna}

Many commercial cellular systems deploy Omni-type antennas, for the most part, in an area where the traffic load is low and coverage area is not of great concern to the operator. The main benefit to these antennas is the little planning needed for a rapid deployment of a cellular system. In terms of RF propagation, the antenna transmits equally in a $360^{\circ}$ area around the antenna, as illustrated in Figure 6-1, which makes planning the cellular systems easier. The transmitting and receiving pattern decreases the coverage area, which will limit the users allowed on the system.

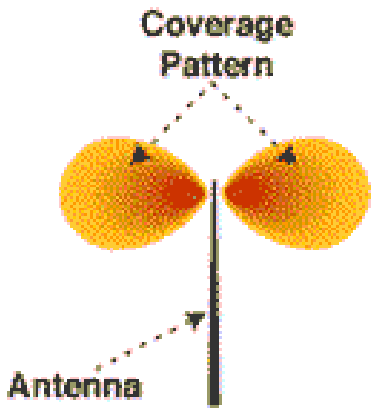

Side View

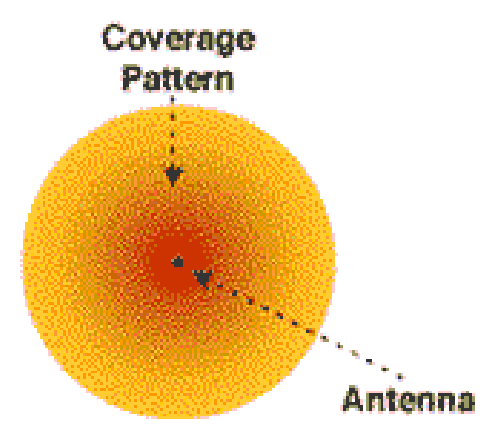

Top View

Figure 6-1: Omni Antenna Coverage Pattern [91]

Omni antennas for the ACN are in the form of a blade-type of antenna, as shown in Figure 6-2. The key benefit to using the omni blade antenna is the relative ease at which they can be installed on the aircraft. The deployment of the blade antenna would require two separate antennas: one for transmit and receive and one for receive diversity. Spacing of the antennas would depend on the frequency deployed, typically 3-5 meters. 
The Omni blade antenna, as shown in Figure 6-2 has been used in previous trials when deploying cellular communications on aircraft, specifically the Battle Field Airborne Communications Node (BACN). This series of tests, conducted by Northrop Grumman, used two Omni antennas for transmit and receive. One of the key findings in their report is to look into directional antennas to optimize the coverage area and link budget. [94]

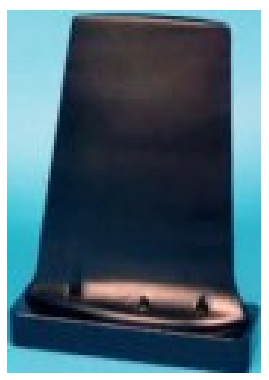

Figure 6-2: Blade Antenna

Although this type of antenna is easy to deploy and is inexpensive, the coverage and capacity will not be optimized for large traffic volumes. Since the recommended flight path is either a circular or racetrack pattern with the coverage area in the center of the pattern, up to $60 \%$ of the transmit energy from the antenna will be lost. Another draw back for this type of antenna is the possibility of interfering with commercial ground systems since the direction in which the signal is transmitted cannot be controlled. As was previously mentioned, one of the concerns of the ACN is to limit the interference into operating systems.

\subsubsection{Sector (Directional) Antennas}

Sectorized or directional antennas are used in almost all cellular systems due to the ability to increase the capacity of these systems. Conventional directional antennas divide the cell into a given number of sectors, depending on the type of antennas. Typically, the cells are divided into $120^{\circ}$ ( 3 sector) or $60^{\circ}$ (6 sectors) to increase the coverage and capacity over an Omni-type configuration, as shown in Figure 6-3. Usually these antennas act as an interface between the base station and RF environment, and do not have beam steering or smart capability. The main advantage to the sector antenna is there are gains in capacity and coverage due to the concentrated power, when compared to the Omni antennas as can be seen in Figure 6-4. There is some planning for down tilt that needs to be completed prior to installation in order to position the antennas the proper way to achieve the desired coverage. For the ACN, certain considerations will need to be made concerning aircraft bank angle, antenna positioning, and flight route.
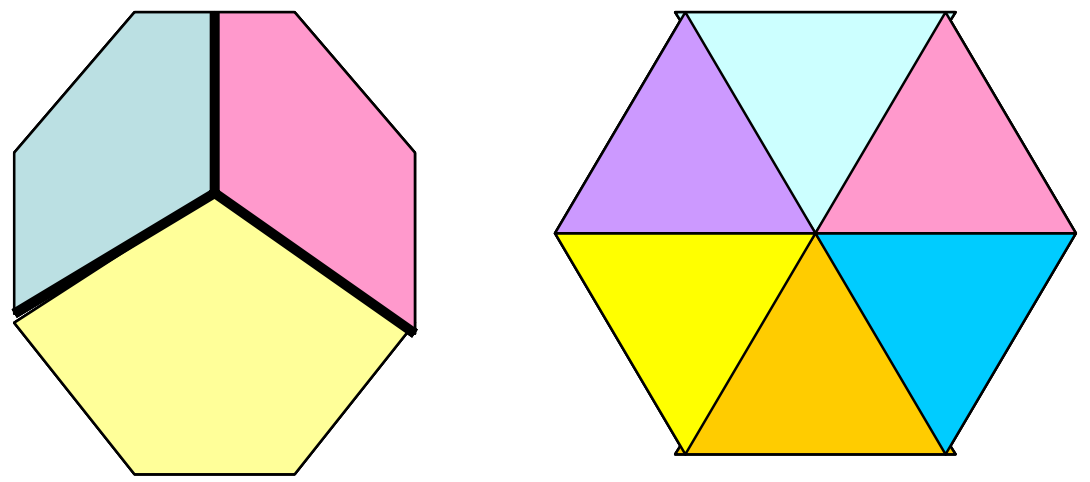

Figure 6-3: Three (Left Figure) And Six (Right Figure) Sector Configurations 


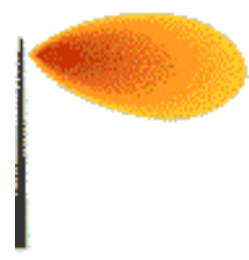

Side View

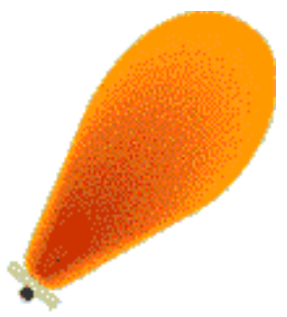

Top View

Figure 6-4: Directional Antenna Coverage [91]

Directional antennas are a viable option for the ACN platform. There are many ways that the antennas can be mounted and deployed in the different scenarios. Many antenna suppliers manufacture flat panel antennas that can be mounted in aircraft. These types of antennas can have a beam width between $30^{\circ}$ and $120^{\circ}$, which would be ideal for the ACN application.

\subsection{Specialized Antenna Configurations}

Specialized antenna systems are becoming increasingly popular with cellular communications carriers as cellular applications evolve and user preference changes. Switched Beam antennas, Adaptive Smart antennas, polarized diversity antennas, and software driven antenna systems are all configurations that are currently deployed by network operators to enhance their coverage and capacity. They also contain multiple elements. Some antenna systems have DSP that perform complex calculations to determine the angle of arrival, signal strength, and other metrics. Other antenna systems are configured in such a way that they divide a sector into portions and transmit the energy in beams to specific points in the coverage area using less-complicated weighting factors. Since the antennas consist of multiple elements, they have the capability to concentrate power on one or more elements, depending on where the most traffic is located. Usually the method and algorithms reside in the base station, but in some cases, the antenna may contain the hardware and software to make these determinations.

\subsubsection{Switched Beam Antennas}

Switched Beam antennas, as show in Figure 6-5, may be utilized in an Omni configuration or as sectors. Switched Beam antennas contain many elements that are spaced at a predetermined wavelength apart. For $800 \mathrm{MHz}$ cellular systems, this spacing is typically 0.6 wavelengths. [92] The antenna may be manually "steered" by providing a null signal to the received signal on certain elements, thus increasing the SNR to provide coverage in certain areas, or a mathematical algorithm could steer the beams from the appropriate elements when loads increase on that element. 


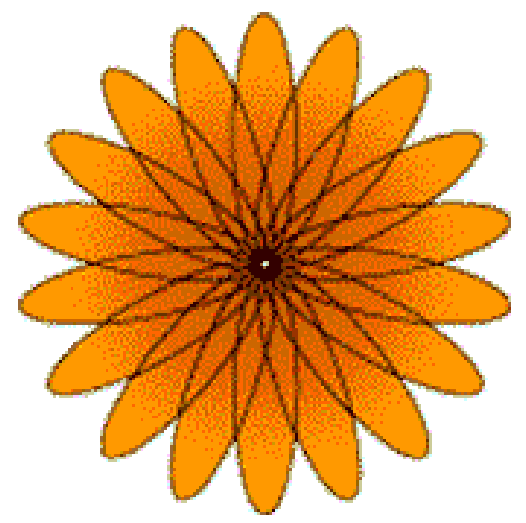

Figure 6-5: Switched Beam Smart Antenna Coverage Area (Omni) [91]

Another type of multi-element Switched Beam antenna is purely configuration driven with comparatively simple software algorithms. This type of antenna system is unique because instead of relying on antenna nulls or the angle of arrival on a certain element, it relies on chip delay or advance when compared to system time. In Figure 6-6, a typical $120^{\circ}$ sector is divided into three separate logical sectors using three elements in a panel-type of antenna. The term logical sector is used even though there are three physical elements, since the base station treats the antenna system as one sector in its database. The CDMA pilot signal is modified by two chips by either delaying or advancing the chips on either side of center. This pilot advance or delay causes the mobile to change it's transmit signal (advance or delay) when communicating with the base station. The base station then correlates the received signal to the element or logical sector for processing. For example, if there is a high volume of calls in the +2 Chip portion of the sector, the base transceiver station BTS will increase sensitivity in that area to increase capacity. As the call load transitions through the sector, the chip increment will change and the BTS will change or steer elements to maintain capacity. This implementation is useful when call loads change within a sector, and those call load changes are predictable. This technology has been deployed in high-density areas where there are many mobile users.

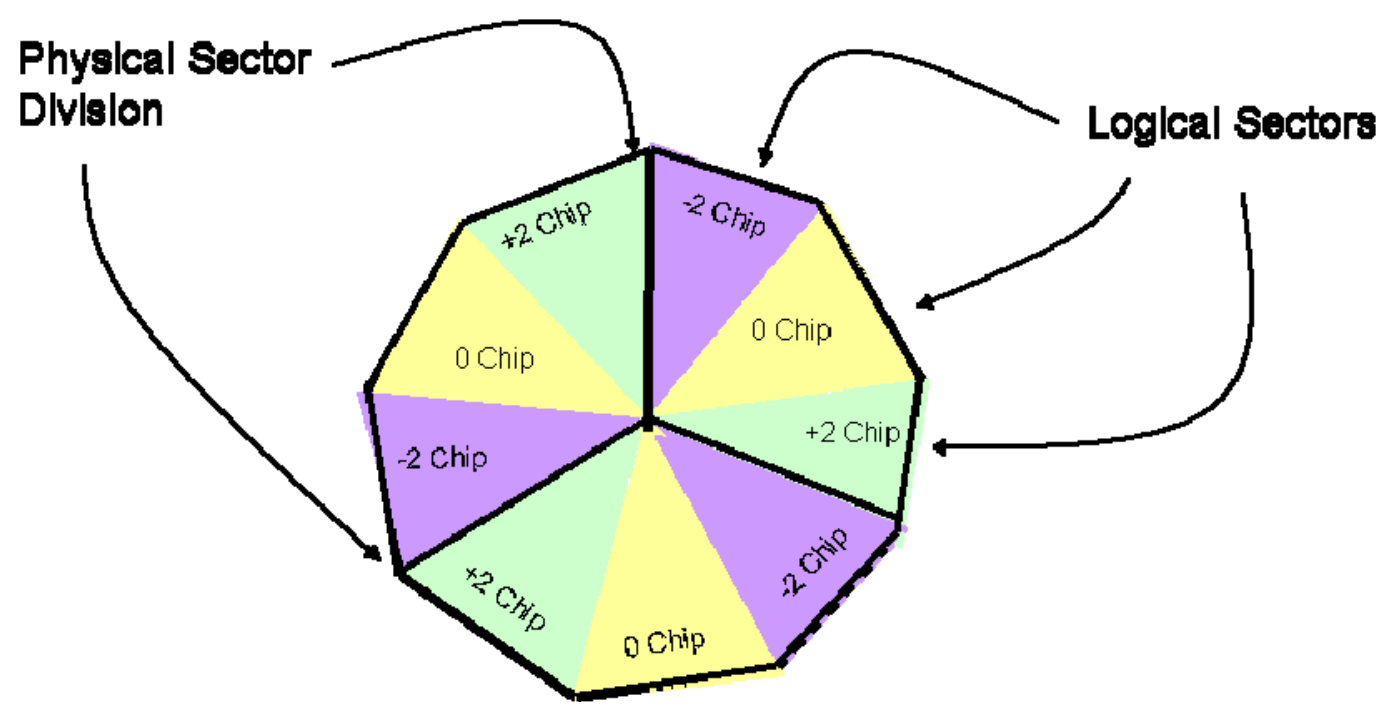

Figure 6-6: Typical Switched Beam Smart Antenna Configuration

The implementation discussed in the previous paragraph will only work with CDMA technologies due to the nature of the pilot signal in CDMA systems. There may be similar implementations for the 
GSM technologies, but due to the delay and Doppler Effect of the ACN platform, this type of smart antenna would realize little of the intended gains.

\subsubsection{Adaptive Antennas}

Adaptive Antennas, sometimes referred to as smart antennas, are similar to Switched Beam antennas, with regards to having multiple elements and being more efficient that typical sector antennas. The key difference between the two types of antennas is that adaptive smart antennas use spatial and temporal algorithms to adjust their directional patterns in order to maximize the SNR. [93] In other words, the antenna can adapt to the environment through software. Figure 6-7 shows an example of an adaptive antenna. Notice how a lobe is concentrated on the user, and null energy is given to the interferer. This is extremely effective and efficient in highly loaded sectors where the users are concentrated in a certain area.

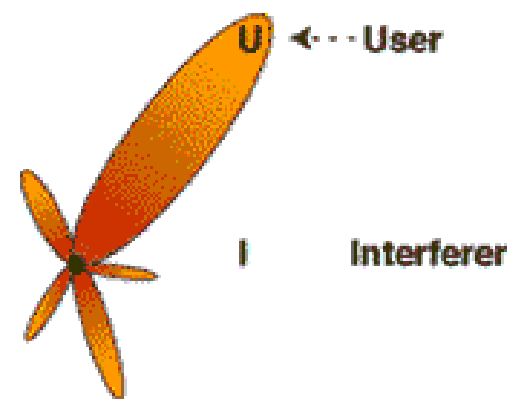

Figure 6-7: Adaptive Smart Antenna Coverage Area [91]

Figure 6-8 shows the coverage comparison of the three main types of antennas typical in current cellular systems. The coverage gains are approximately 2 times for conventional systems and $33 \%$ for Switched Beam in a low-interference environment. In a high-interference environment, such as a heavy call load, note that while conventional and Switched Beam antenna systems actually decrease the coverage area, the adaptive antenna is relatively the same as in a low interference environment. This is due to the adaptive system's ability to "ignore" the excess noise and concentrate on the needed signal.
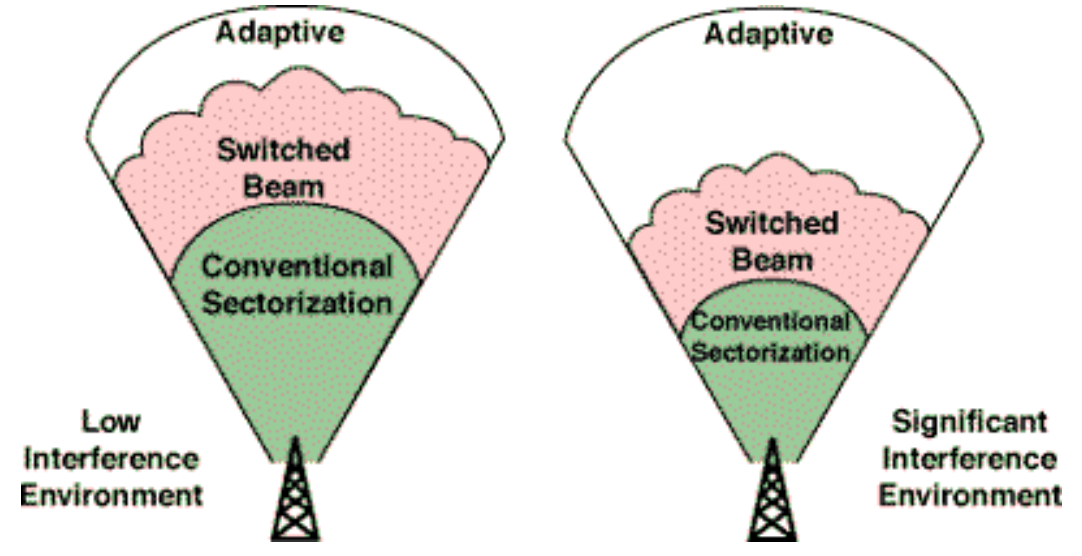

Figure 6-8: Antenna Sensitivity Comparison [91]

There are several ways to implement adaptive antennas using combinations of hardware and software. The most common and easy way to deploy them is to add spatial and temporal processing equipment between the phased array antenna and the base station. Usually an add-on card to the base station is available that takes the antenna inputs or outputs, process the signals, and then send the signal 
to the normal paths throughout the base station. This equipment is not technology dependent, so there are no configuration differences between CDMA and GSM.

Table 6-1 quantifies the benefits of an Adaptive antenna when compared to a traditional antenna. As is shown in this comparison, the Adaptive antenna dramatically outperforms the traditional antenna.

Table 6-1: Benefits of Smart Antennas [91]

\begin{tabular}{|c|c|}
\hline \multicolumn{1}{|c|}{ Feature } & Benefit \\
\hline $\begin{array}{c}\text { Signal gain. Inputs from multiple } \\
\text { antennas are combined to } \\
\text { optimize available power } \\
\text { required to establish given } \\
\text { level of coverage. }\end{array}$ & $\begin{array}{c}\text { Better range/coverage. Focusing the energy into the cell } \\
\text { increases base station range and coverage. Lower power } \\
\text { requirements also enable a greater battery life and } \\
\text { smaller/lighter handset size. }\end{array}$ \\
\hline $\begin{array}{c}\text { Interference rejection. Antenna } \\
\text { pattern can be generated } \\
\text { toward co-channel interference } \\
\text { sources, improving the signal- } \\
\text { to-interference ratio of the } \\
\text { received signals. }\end{array}$ & $\begin{array}{c}\text { Increased capacity. Precise control of signal nulls, and } \\
\text { mitigation of interference combines to decrease frequency } \\
\text { reuse and reduce distance (or cluster size), improving } \\
\text { capacity. Certain adaptive technologies (such as space } \\
\text { division multiple access) support the reuse of frequencies } \\
\text { within the same cell. }\end{array}$ \\
\hline $\begin{array}{c}\text { Spatial diversity. Composite } \\
\text { information from the array is } \\
\text { used to minimize fading and } \\
\text { other undesirable effects of } \\
\text { multipath propagation. }\end{array}$ & $\begin{array}{c}\text { Multipath rejection. Can reduce the effective delay spread of } \\
\text { the channel, allowing higher bit rates to be supported } \\
\text { without the use of an equalizer. }\end{array}$ \\
\hline $\begin{array}{c}\text { Power efficiency. Combines the } \\
\text { inputs to multiple elements to } \\
\text { optimize available processing } \\
\text { gain in the downlink (toward } \\
\text { the user). }\end{array}$ & $\begin{array}{c}\text { Reduced expense. Lower amplifier costs, power consumption, } \\
\text { and higher reliability will result. }\end{array}$ \\
\hline
\end{tabular}

\subsection{Diversity Antennas}

Diversity in cellular systems is most commonly used in the receive path of the reverse link (i.e., the base station receive). Additionally, certain types of mobiles utilize receive diversity on the forward link, but these mobiles are manufacturer specific and not technology specific. Receive diversity is important due to the non-LOS nature of cellular communications systems and the multipath signals that are generated by reflection and refraction of these signals. As these multipath signals null each other out, the signal is said to be in a fade, which can cause poor voice and data qualities. If the multipath interference is out of phase enough, the fade is considered to be deep. Deep fades typically result in unrecognizable voice communications or lost calls. Receive diversity helps reduce the incidence of deep fades by a factor of 10. Research of cellular systems show that without any form of receive diversity a cellular call is in a deep fade $10 \%$ of the time. These deep fades happen at nominal speeds with otherwise good signal levels. Once receive diversity was added to the system, deep fades were reduced to only $1 \%$ of the time, thus greatly improving call quality and duration. [94] Since it cannot be guaranteed that the commercial handsets will have receive diversity, the ACN should be designed with its own receive diversity system. This can be either spatial diversity, which is common in most commercial cellular systems, or polarization diversity, which is an emerging technology for commercial systems. 


\subsubsection{Spatial Diversity}

The most common type of diversity receive employed in cellular systems is spatial diversity. Spatial diversity is accomplished by separating the antenna by a predetermined distance, usually 10 to 30 wavelengths horizontally. [95] Spatial diversity antennas typically use vertically polarized antennas that effectively overcome deep fades by comparing and combining the two received signals, and using the highest quality received signal. Since the antennas are separated, it is likely that only one receive antenna, either the main receive or diversity receive, will be affected by the faded signal due to the multipath delays. As the example shows in Figure 6-9, left antenna is the main receive, the center is transmit, and the right antenna is receive diversity.

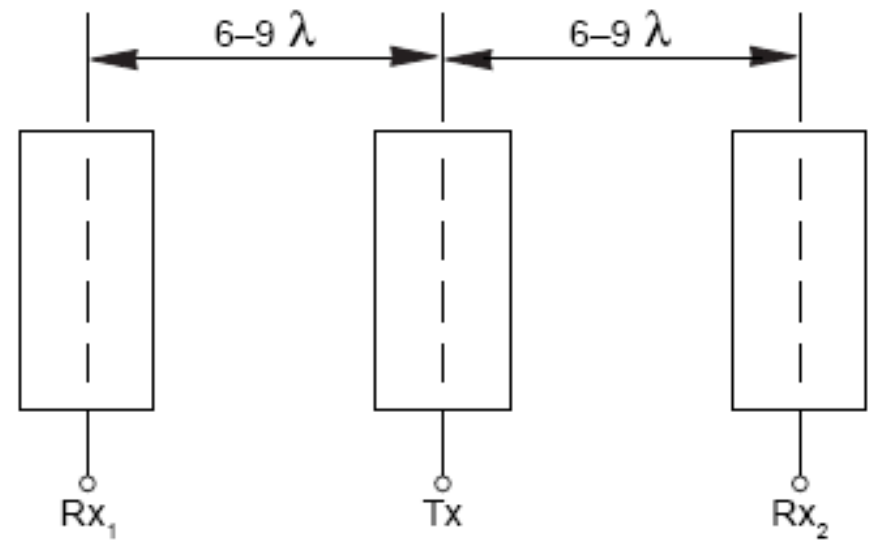

Figure 6-9: Spatial Diversity Antennas

\subsubsection{Polarization Diversity}

Another type of receive diversity is polarization diversity. Polarization diversity is a system that uses both horizontal and vertical elements to increase the receiver gains, as show in Figure 6-10. Recent studies have proven that one of the best ways to realize the benefits of diversity is to use polarization diversity. [93] This is due to the nature of which the common mobile user holds their phone. In early cellular systems, the elements of antennas were primarily vertically polarized, since the majority of the antennas were mounted on vehicles with a vertical polarization. The base station antennas were vertically polarized, therefore creating the need for two vertically polarized antennas. Current studies have shown that most cellular mobile stations are handheld, usually at a $60^{\circ}$ angle relative to vertical. This difference in orientation creates wave fronts on both the vertical and horizontal axis, thus creating the need for a horizontal element for receive.

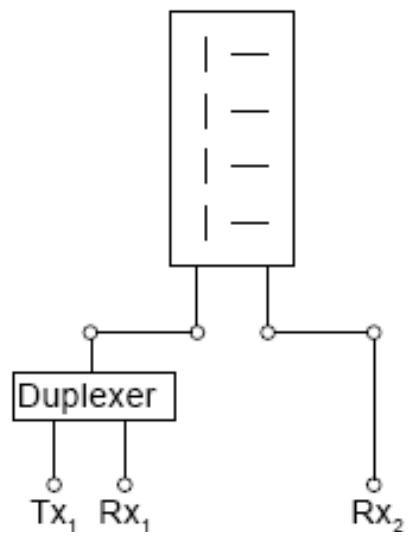

Figure 6-10: Polarization Diversity Antenna 


\subsubsection{Cross Polarization Diversity}

The last method to achieve the benefits of receive diversity is through cross polarization diversity. This can be accomplished by using cross-polarized antennas (X-POL). X-POL antennas have elements that are offset $45^{\circ}$ from the vertical axis and can accept dual inputs using a duplexer, as shown in Figure 6-11. This type of receive diversity scheme produces the same gains as the horizontal/vertical polarization diversity antennas. The key benefit of this type of antenna is that there can be only one antenna for multiple transmit and receive paths. [96] X-POL diversity may be extremely beneficial to the ACN configuration due to the rapid movement of the aircraft and the movement of ground base mobile stations.

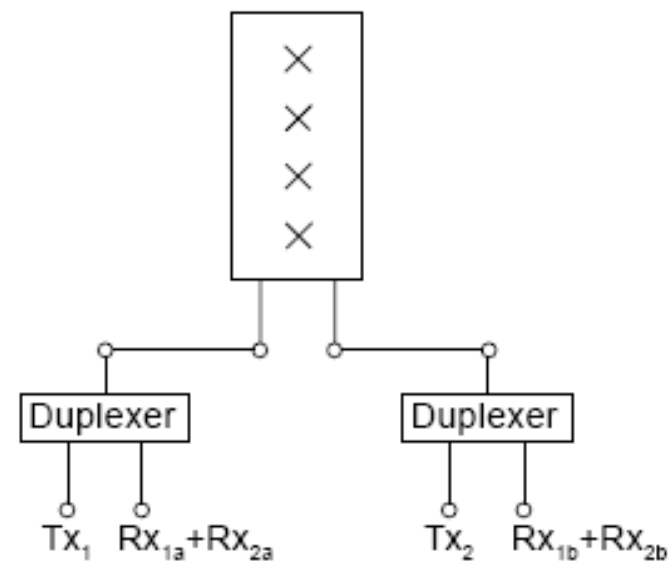

Figure 6-11: Dual Transmitter/Receiver X-POL Antenna

\subsection{Land Mobile Radio Antennas}

Land mobile radios (LMR) are the 2-way radios that most first responders use in an emergency. These radios typically operate in three bands, $150 \mathrm{MHz}, 450 \mathrm{MHz}$, and $700 \mathrm{MHz}$ range, but there should be consideration for other bands since it cannot be guaranteed that all disaster areas will use the same frequencies. Since most LMR systems are extremely tolerant to delay, the only considerations needed for LMR systems are frequency and coverage.

Antenna size for the $700 \mathrm{MHz}$ range should not be of concern. Since these antennas are typically small enough, there is no impact on flight. Different methods, other than the typical antennas used for lower frequencies, will need to be deployed for $150 \mathrm{MHz}$ and $450 \mathrm{MHz}$ systems. These antennas will either need to be of the loop-type or a bended element so that the antenna is still capable of receiving the lower frequency and transmitting at a high power, but small enough to be mounted to the platform, some options are shown in Figure 6-12. Many of these antenna systems are currently in use, with the applications usually in dense populations and urban areas. 


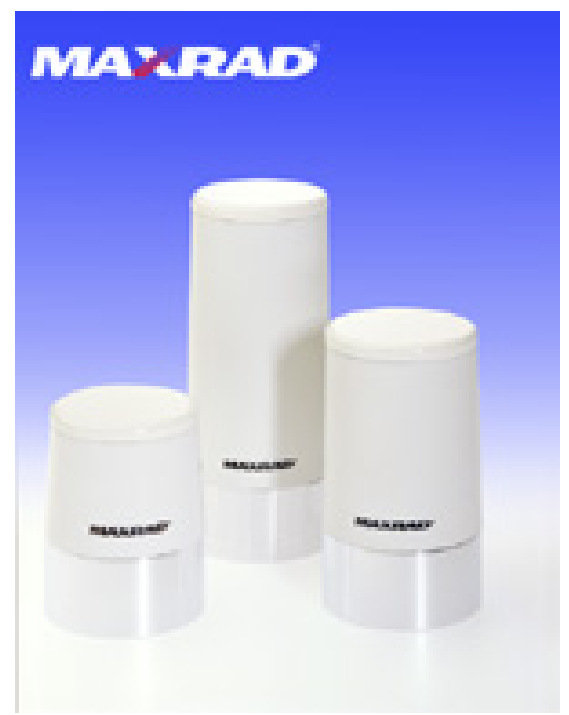

Figure 6-12: Low Profile Coiled Land Mobile Radio Antennas

Finally, the determination should be made if the antenna should be Omni or directional. The argument for Omni antennas can be made since not only first responders in the disaster area will need to communicate with each other, but they will need to communicate with responders and coordinators on the outside of the disaster area. Using an Omni antenna would indeed provide this capability. On the other hand, there should be a concentrated system that the first responder can use without competing with outside users. This is the case for a directional antenna. The final configuration for the antenna lies more in the way the infrastructure is designed for capacity, and if it can handle a wide user base. If there is ample equipment for many users, an Omni antenna could be used. If there are limited channels, then a directional antenna will need to be used.

\subsection{Antenna Mounting Techniques}

Antenna mounting on an aircraft can be a particularly arduous task. The primary consideration is whether to mount the antennas permanently or in a temporary fashion to make the ACN concept as flexible as possible. Other constraints, such as FAA certification, expense, and technology availability, need to be taken into consideration when determining the best way to mount the antennas. Permanent mounting is used when the system is resident to the platform and the antennas will not be taken off. Temporary mounting options will be used when the system is not dedicated to a specific platform or airframe.

\subsubsection{Permanent Mounting Techniques}

Permanent mounting should be considered as a viable solution to the ACN. For the purposes of the ACN, permanent mounting means the antennas will not be removed from the aircraft. Additionally, certain airframe modifications will need to be made, which could mean increased expense and modification time.

The most costly way to permanently mount antennas is to modify the aircraft fuselage to accept a new antenna system, which can be a very involved task. Since the antenna positioning will need to be in an exact position in the fuselage, cabling, wires, hydraulics, and structural components will need to be relocated. Additionally, environmental factors, such as cabin pressurization, will need to be verified. Since this changes the integrity of the airframe, an FAA inspection will be required. 
Another, less costly option, is to use existing antenna positions and install antennas capable of transmitting and receiving cellular signals, such as Omni blade antennas. For example, the RC-135 is currently equipped with steerable beam antennas that are pre-positioned to provide ample cellular coverage in either the circular route or racetrack pattern. These antennas are receive-only, and therefore not capable of transmitting cellular signals. If these antennas are replaced with permanent transmit capable antennas, no airframe modification is needed. During the aircraft's intended mission, the antennas would be in receiving mode, but when the cellular system is needed, they could be switched to transmit and receive. Figure 6-13 describes this concept.

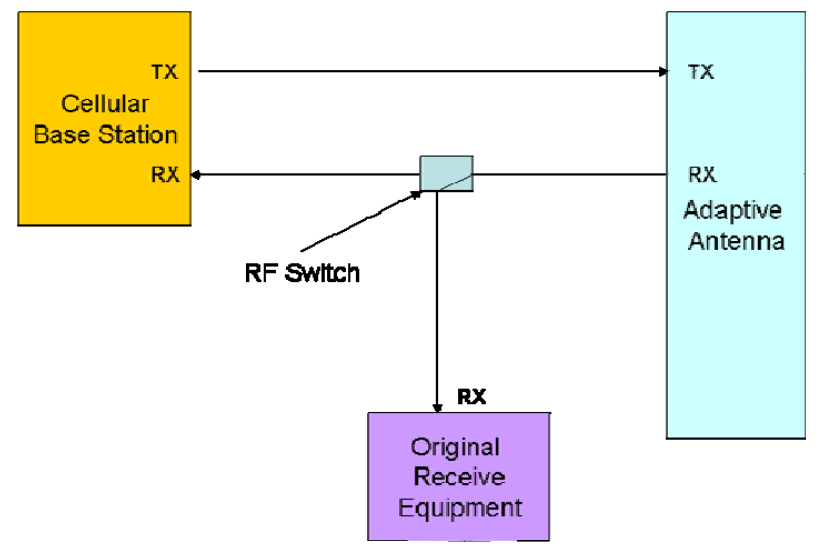

Figure 6-13: Dual-Mode Antenna.

Finally, most cost effective way to mount the antennas permanently is to use the existing antennas in the configuration that they are normally used. An example of this would be to use existing blade antennas, assuming they are frequency matched. The utilization of these antennas would be ideal for first responder communications using a land mobile radio system since these systems are typically single antenna systems. Conversely, this configuration would not be ideal for cellular communications due to the need for receive diversity.

\subsubsection{Temporary Mounting Techniques}

Temporary mounting is a cost effective way to deploy antennas on the ACN. It also increases the flexibility of the ACN due to the ability to equip any platform for any mission. Typically, temporary mounting will not cause changes to the airframe, thus reducing cost and turn around time.

One way to mount the antennas temporarily is to use a false door that contains the antennas. The false door would be manufactured to lock in an existing doorframe of a removable or upward opening door. The false door would have flat panel antennas mounted in it that would have mechanical tilt to offset the bank of the aircraft. The aircraft would not be able to be pressurized since the door would not be a structural component, which may limit the operating altitude of the platform. With this implementation, a fleet of aircraft could use that same false door, assuming any aircraft in the fleet could receive the other communications equipment.

Another mounting technique for temporary antennas is the communications pod. Small aircraft architecture is best contained in a single pod, which could be mounted to hard points of several different types of aircraft of either fixed wind or rotary wing. The pod would contain lower capacity GSM and CDMA systems and could be outfitted to accept first responder UHF/VHF systems. This system would utilize a configurable backplane that could accept multiple cards or Line Replaceable Units (LRU) achieving flexibility for any type of deployment. 
The architecture is similar to the large aircraft architecture, with the exception of the size and power required to operate the equipment. Since there is limited payload capacity and power available at the hard points of an aircraft, it is necessary to limit the equipment and thus the capacity of the system. It is possible to increase capacity during an event by adding multiple pods to a single aircraft, should it be necessary.

The pod will use next generation adaptive antennas that are integral to the pod, increasing capacity and limiting interference to outside cellular users. Additionally, the system will use telemetry data to determine adjustments in coverage areas as the aircraft turns, climbs, or dives. The pod will also consider future cellular technologies, including CDMA EV/DO and W-CDMA. For backhaul, the system will use satellite links to send signaling data to ground-based switching centers in order to connect to land lines.

The Figure 6-14 depicts a sample of how the pod's internal layout would look. The cards (or LRUs) would be separated by technology in shielded compartments. This would limit interferences when transmitting and receiving on similar frequencies. This layout provides for an internal GPS antenna and an internal backhaul antenna. Both of these capabilities may utilize existing aircraft infrastructure to reduce cost and weight, if needed.

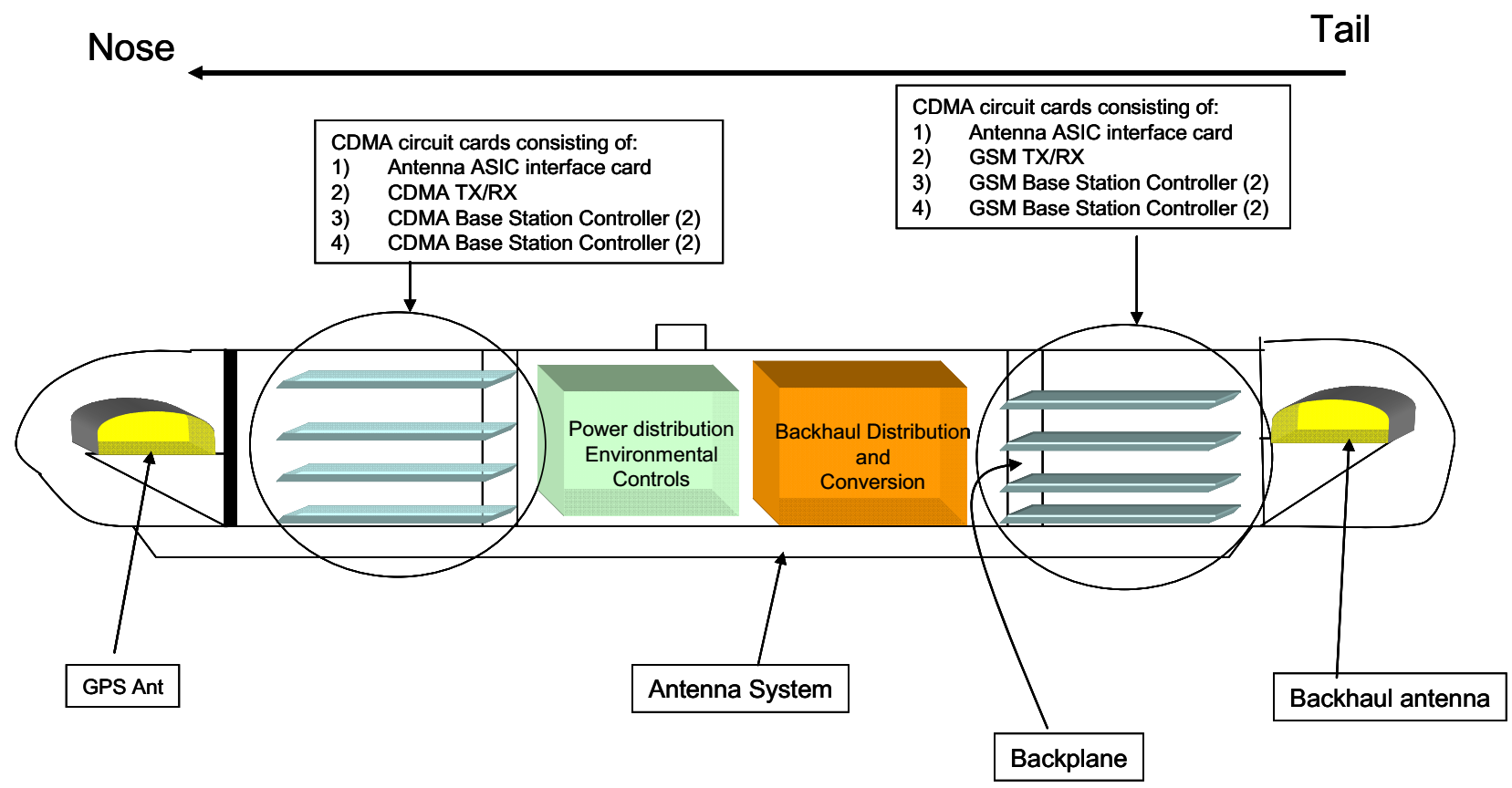

Figure 6-14: Pod Design For Cellular Equipment.

The Figure 6-15 shows the adaptive antenna array. The antenna laminate is integral to the pod structure while the elements are a separate removable piece. The antenna elements are circularly polarized and can be electrically steered with telemetry input to maintain the maximum coverage area. 


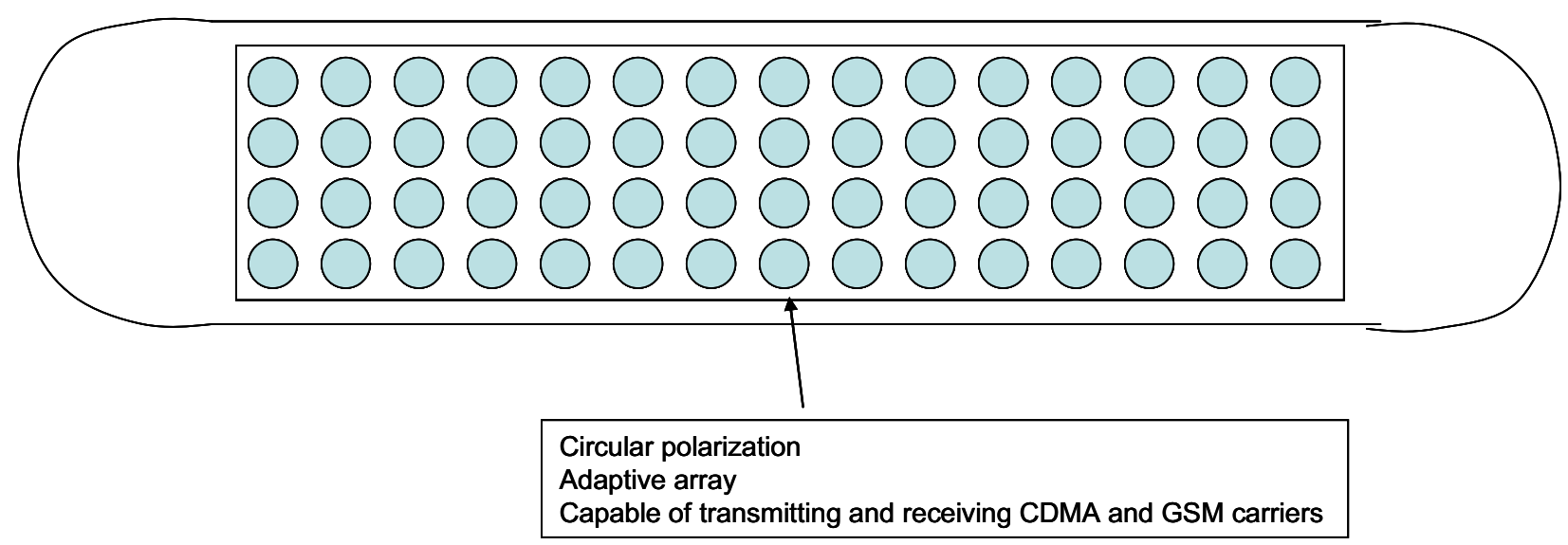

Figure 6-15: Pod Antenna Structure.

\subsection{Antenna Physical Properties}

The physical properties of an antenna are an important factor when determining the type of antenna to use. As part of properly sizing an antenna, weight, drag, frequency, sensitivity, and platform type will all need to be accounted for. Unfortunately, if all of these factors are optimized and put into one antenna system, the final product may be cost prohibitive. It is for this reason that commercial cellular systems often will deploy an antenna that fits local needs. For example, a cellular carrier may choose to deploy a small aesthetically pleasing antenna to comply with local building codes, but may lose valuable gains of a larger antenna that impacts the system. For the ACN application, the previously mentioned variables that affect the size of the antennas are absolute requirements. The ACN will require an antenna that is small, so it reduces drag and weight.

\subsection{Antenna Conclusions and Recommendations}

Although there are many COTS solutions for antennas, the most effective way to provide ideal coverage and capacity is to use a specialized antenna. Throughout this section, different antenna systems have been discussed in detail. From this discussion, the following conclusions have been reached:

- Panel (directional) antennas are ideal for aircraft applications

- Cellular antennas should utilize cross-polarization diversity in order to reduce the antenna footprint while increasing sensitivity

- Antennas should be capable of transmitting more than one carrier frequency

- Due to the gains in coverage area, antennas should be adaptive systems

- Antenna beam width should be such that the horizontal and vertical beam widths are at least $80^{\circ}$

- First responder communications may use an Omni antenna

- First responder communications antenna should be capable of transmitting and receiving in the $150 \mathrm{MHz}, 450 \mathrm{MHz}$, and $700 \mathrm{MHz}$ bands 
- Permanent-mounted antennas will provide the best continuity for planning flight operations

- When using temporarily mounted antennas, mounting configurations should be standardized to provide continuity with flight planning operations.

- Table 6-2, provides all of the antennas discussed in this section for comparison. As the table shows, there are benefits and drawbacks to each type of antenna.

Table 6-2: Antenna Comparison

\begin{tabular}{|c|c|c|c|c|c|c|c|}
\hline & \multicolumn{6}{|c|}{ Parameter } & \\
\hline \multirow{6}{*}{ 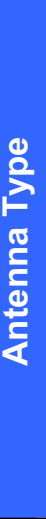 } & & $\begin{array}{c}\text { Cost } \\
(1=\text { Most } \\
\text { Expensive, } \\
5=\text { Least } \\
\text { Expensive) }\end{array}$ & $\begin{array}{l}\text { Installation } \\
(1=\text { Most } \\
\text { difficult, } \\
5=\text { Easiest) }\end{array}$ & $\begin{array}{l}\text { Footprint } \\
\text { (on Aircraft) } \\
\text { (1 = Largest, } \\
5 \text { = Smallest) }\end{array}$ & $\begin{array}{c}\text { Capacity } \\
(1= \\
\text { Worst, } \\
5 \text { = Best) }\end{array}$ & $\begin{array}{c}\text { Coverage } \\
\text { (1 = Worst, } \\
5=\text { Best) }\end{array}$ & Grade \\
\hline & Omni & 5 & 4 & 3 & 2 & 2 & 16 \\
\hline & Directional & 4 & 3 & 2 & 3 & 3 & 15 \\
\hline & X-POL & 3 & 4 & 5 & 4 & 4 & 20 \\
\hline & Switched Beam & 3 & 2 & 4 & 4 & 4 & 17 \\
\hline & Adaptive & 2 & 2 & 4 & 5 & 5 & 18 \\
\hline
\end{tabular}




\section{REFERENCES}

[89] Polakos, Paul, Lucent Technologies, "Intelligent Antennas and BLAST for CDMA2000 Networks,"

http://www.cdg.org/news/events/CDMASeminar/cdg_tech forum_02/3 lucent ia blast final_release .pdf, Web page accessed 07/07.

[90] Andrew Antenna Application Notes, http://www.andrew.com/products/antennas/bsa/catalog29/14 Application.Engineering\%20Notes.pdf, Web page accessed 10/07.

[91] International Engineering Consortium, "Smart Antenna Systems," http://www.iec.org/online/tutorials/smart ant/topic01.html, Web page accessed 10/07.

[92] Fujimoto K., Jame, J. R., "Mobile Antenna Systems Handbook,” Artech House, 2001, pp. 208221.

[93] Hudson, J. E., “Adaptive Array Principles,” Peregrinus LTD., 1991, pp. 1-19.

[94] Drucker, Elliot, "Downlink Receive Diversity: Something for (Almost) Nothing," Wireless Week, August 16, 2006.

[95] Aydin L., Esteves E., Padovani R., "Reverse Link Capacity and Coverage Improvement for CDMA Cellular Systems Using Polarization and Spatial Diversity” IEEE, 2002.

[96] Kathrein Application Notes, "Dual Polarized Base Station Antennas for Polarization Diversity Systems," 2005.

[97] Northrop Grumman “BACN CDMA Subsystem Test Results,” June 02, 2006, p. 13. 


\section{ACRONYMS}

$\begin{array}{ll}\text { ACN } & \text { Airborne Communications Node } \\ \text { BACN } & \text { Field Airborne Communications Node } \\ \text { CDMA } & \text { Code Division Multiple Access } \\ \text { COTS } & \text { Commercial Off-the-Shelf } \\ \text { DSP } & \text { Digital Signal Processors } \\ \text { GSM } & \text { Global System for Mobile } \\ \text { LMR } & \text { Land mobile radios } \\ \text { LOS } & \text { Line Of Site } \\ \text { LRU } & \text { Line Replaceable Units } \\ \text { MIMO } & \text { Multiple Input Multiple Output } \\ \text { OFDM } & \text { Orthogonal Frequency Division Multiplexing } \\ \text { SNR } & \text { Signal to Noise Ratio }\end{array}$




\section{Coverage}

When contemplating the deployment of an Airborne Communications Node (ACN), there are several constraints that should be considered. Aircraft endurance, Doppler limitations, speed of the aircraft, and antenna positioning are the physical constraints that are taken into account throughout this section of the report. Additionally, more abstract constraints (e.g., distant cell interference, RF spill over, and individual technology constraints [delay, Doppler Effect, and distance]), should be considered when planning the coverage capabilities of the ACN.

This section discusses the variables that need to be considered when determining the coverage area of the ACN. The different types of coverage areas - either circular or racetrack - are discussed along with the constraints (e.g., Doppler Effect, cell breathing, and path loss). Finally, five coverage examples are given for common platforms that may be deployed as an ACN.

\subsection{Impacts of Changing Flight Characteristics on the Coverage Model}

When planning the coverage areas, knowing the capabilities of the aircraft and its flight characteristics are a critical requirement. Simply stated, how high, fast, and long an aircraft can fly determines antenna positioning, coverage areas, and the endurance of rescue operations. One change in any of the flight parameters affects all of the ACN's ability to cover the required area.

Aircraft altitude is the first characteristic that has broad reaching effects on the ACN mission requirements. As the operating altitude of the platform changes, either the coverage model changes or the antenna deployment changes, which affect the coverage of the system. To determine how altitude affects coverage area, three thresholds will need to be maintained: Maximum Path Loss, Angle of Arrival (to mitigate Doppler Effect), and Maximum Delay. For example, in a $1500 \mathrm{sq} / \mathrm{km}$ coverage area, as the aircraft altitude increases, the path loss and delay increase, but the Doppler Effect is lowered. Conversely, as the altitude is lowered, the path loss and delay decrease while the Doppler Effect is increased. Since GSM is the limiting technology on this platform, all examples will use those thresholds to determine coverage area. Table 7-1 shows the maximum thresholds for path loss in terms of Received Signal Strength Indication (RSSI), Doppler shift, and delay.

Table 7-1: Key GSM Thresholds

\begin{tabular}{|l|c|}
\hline \multicolumn{1}{|c|}{ Output } & Limit \\
\hline Doppler Shift (Hz) & 412 \\
\hline RSSI (Mobile) (dBm) & -90 \\
\hline $\begin{array}{l}\text { Delay (Cell Range) } \\
\text { (km) }\end{array}$ & 35 \\
\hline
\end{tabular}

Aircraft speed is another characteristic that will need to be taken into account when planning coverage areas. Aircraft speed may have an adverse affect on cellular performance due to Doppler Effect. As the aircraft speed is increased, the more profound the effects the Doppler shift will have on the cellular communications. There are a couple of ways to increase speed and mitigate the effects of Doppler shift, one of them being to offset the aircraft from the coverage area. 


\subsection{Different Flight Routes}

There are numerous ways to plan flight routes, all of which provide varying degrees of coverage. Several different flight paths were considered during the ACN analysis and the determination was made to narrow both the study and the simulation to either a racetrack or oval pattern and a simple circular pattern. The key reason for this determination is the fact that with these two patterns, coverage times are extended and maintained. In more typical fly over patterns, the coverage time is limited, thus limiting the amount and quality of service that can be provided.

\subsubsection{Circular Flight Route}

The circular flight route should be considered when planning due to the numerous benefits that it offers, including consistent coverage, ease of planning, and rapid deployment. The circular route gives the best single-sector coverage because this flight path allows the RF energy or beam to be projected over the coverage area constantly. Using this route, it is relatively easy to plan for Doppler Effect, path loss, and delay due to the constant velocity, and antenna angle. It also simplifies the planning process for determining how large the deployed asset's coverage area can be.

There are some drawbacks to using the circular route - mainly station keeping and reduced coverage. Station keeping may be difficult due to the speeds of winds aloft, as well as the constant bank that is required to maintain coverage, in this case anywhere from $2^{\circ}-10^{\circ}$. The reduced coverage happens due to the nature of the circle. Since the circle is limited due to the turning radius of the aircraft, the coverage area shrinks or expands depending on the platform used. All of the examples in this section are of the circular coverage area, and the shrinking and expanding can be seen there.

\subsubsection{Racetrack Flight Route}

The racetrack or oval flight route is optimized for a multisector deployment and using this route allows for greater coverage over a sustained period. Additional coverage is gained by adding the sectors to the system. Not only does adding sectors help coverage, but it also adds capacity to the system. These gains are seen since the platform has the antennas pointed to the inside of the oval to cover the area of interest. This route is more difficult to plan due the need to determine where antennas are positioned, the Doppler Effect on the communications, and outage times at the ends of the route. Additionally, the racetrack flight route is easier to fly due to the relatively straight path that is followed. 


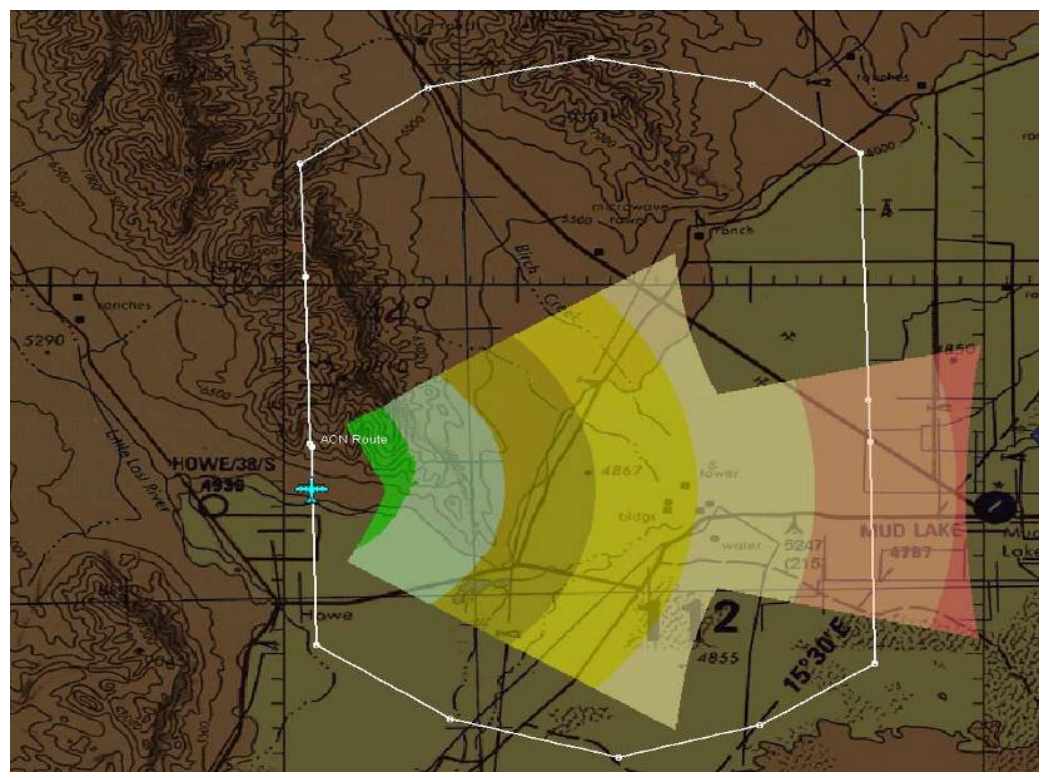

Figure 7-1: Racetrack Flight Path and Coverage Area

\subsubsection{Multiple Aircraft}

It is possible to deploy multiple aircraft in a disaster area to increase either coverage or capacity. Either the circular route or the racetrack route can be used, but there are certain factors that will need to be taken into consideration when planning the scenario. First, and most importantly, aircraft separation will need to be planned. Aircraft separation is important not only for the safety reasons associated with it (collision avoidance, route de-confliction), but there is also a domino effect on coverage and capacity. Once the appropriate separation is decided the coverage area and capacity will have to be determined for that particular altitude. This is important because as the altitude increases or decreases, the coverage and capacity will also increase or decrease.

\subsection{Antenna Positioning}

Antenna placement is important when maximizing the coverage area and capacity of the multisector system. Figure 7-2 and Figure 7-3 show conceptually how antennas may be deployed on the $\mathrm{ACN}$ in a multisector configuration. For a single sector, the two outside antennas would be removed and the horizontal beam width of the remaining sector would be opened up. Figure 7-2 shows the horizontal beam width that was used when predicting the coverage areas for the ACN while Figure 7-3 shows the antenna down tilt in relation to the wing axis. Additionally, the vertical beam width can be seen in Figure $7-9$

\subsubsection{Horizontal Beam Width}

Horizontal beam width is important when planning the coverage area of the ACN. The horizontal beam width of the antenna will control the size of the cell or sector by increasing or decreasing the radiation pattern. The horizontal beam width will also be a determinant for the capacity of the cell or sector by narrowing, and thus increasing received power for all mobiles. A good example is to compare the antennas radiation to a spotlight. A spotlight can project a beam far away because the mirror behind the light focuses and reflects the beam so that is it concentrated. If that mirror was flattened, the beam would lose the intensity and concentration due to the dispersion of the light to the sides. The beam would lose some of its projection strength, and therefore be seen at a lesser distance. The same holds true for antennas and radiation patterns. 
Unfortunately, as the horizontal beam width of the antenna is limited, while increasing (concentrating) the perceived power of the receiver, there will be limits to that antenna's receiving capability. Similar to a horse with blinders, the antenna will only "see" what is inside the horizontal beam width. Figure 7-2 shows the recommended horizontal beam width for cellular systems. This beam width is used in the coverage models that were performed as part of this study. The horizontal beam width, shown in the figure, provides the optimum coverage while minimizing spillover to operating commercial sites.

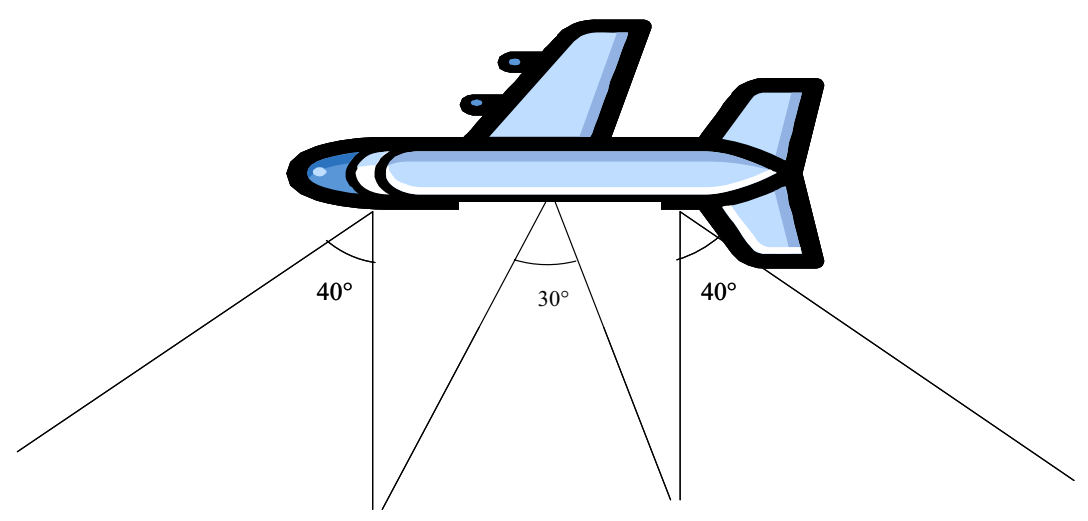

Figure 7-2: Three-Sector Horizontal Beam Width

\subsubsection{Antenna Down Tilt}

Antenna down tilt is an important factor when determining coverage and capacity. The orientation of the antennas is usually placed off a fixed point, in this case the wing. There are a couple of considerations when deciding what the down tilt should be. First, if there is a large coverage area required, there would be less down tilt. By decreasing the down tilt, the antenna is more side looking, therefore increasing the coverage area on the ground. This would increase the radiation pattern on the ground. Altitude also affects the down tilt of the antenna. The lower the aircraft, the down tilt will decrease to optimize the coverage area. The best example would be to use a flashlight to represent the radiation pattern to illustrate how the coverage area changes. If the flashlight is held perpendicular to the ground, there is a circle of light, which would represent the coverage area. As the flashlight is rotated upward, the circle becomes an oblong shape and the light covers more area. This is the same with the radiation patterns of the antenna in relation to the down tilt. Figure 7-3 is an example of how the antennas would be oriented in a three-sector configuration. This pattern is used in the following sections when discussing a multisector configuration. Care should be taken when adjusting the down tilt of the antenna since too little down tilt may cause interference with operating ground sites that are outside of the desired coverage area. 


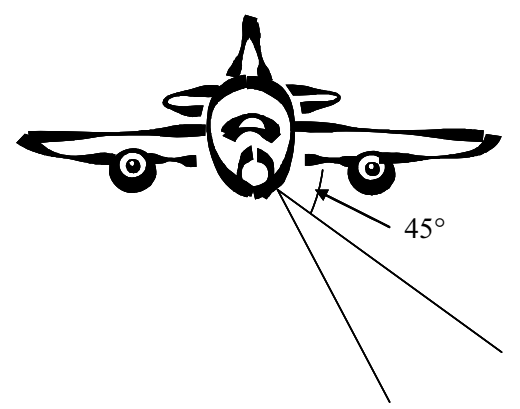

Sectors $1 \& 3$

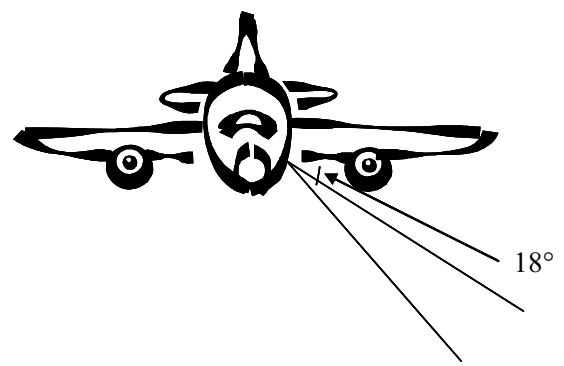

Sector 2

Figure 7-3: Antenna Down Tilt With Relation To Wing

\subsection{Doppler Effect}

Doppler Effect is a phenomenon that will adversely affect the performance of the system if not properly planned for. When comparing the two commonly used cellular communications technologies, GSM and CDMA, the most sensitive to Doppler shift is GSM. The GSM standards require a performance rating of $250 \mathrm{~km} / \mathrm{h}$. This translates into a Doppler shift of $412 \mathrm{~Hz}$; for CDMA, the limit is approximately $750 \mathrm{~Hz}$, which means that CDMA is more tolerant to the frequency shift. It is for that reason sections of this report refer to GSM when discussing the effects of Doppler shift.

Doppler shift occurs when the transmitter or the receiver is in motion. As the receiver moves towards transmitter, the frequency actually increases by potentially several hundred hertz. Likewise, as the receiver moves away from the transmitter, the frequency decreases by several hundred hertz. The most common example of this phenomenon is the police siren. As the siren moves close to the listener at a high rate of speed, the pitch gets higher because the sound waves are being compressed. Once the siren is at a $90^{\circ}$ angle to the listener, the sound is natural because there is no compression. As the siren is moved away from the listener, the pitch of the siren is lower since the sound waves are spread out artificially. The same theory holds true for radio waves, except the change in pitch is replaced by the change in frequency. The result from the shifting frequencies could range from distorted voice to no service.

There are two reasons why GSM is more sensitive to Doppler shift than CDMA. First, the bandwidth of GSM is $200 \mathrm{kHz}$, where the CDMA bandwidth is $1.25 \mathrm{MHz}$. If there is a frequency shift, the affect on a smaller GSM bandwidth is much more pronounced than on the larger CDMA bandwidth. Often, this can lead to out of band transmissions that can cause interference. It is for this reason that one of the ways to mitigate Doppler shift in GSM is to add guard bands. Secondly, the modulation techniques are different for each technology. GSM is predominantly GMSK while CDMA 1X is QPSK. GMSK is sensitive to phase shifts due to the nature of the Gaussian shifting that makes it more difficult for the receiver to differentiate between noise and data. Conversely, CDMA, using QPSK, decodes I and Q data that are more robust in noisy environments.

The simulation model in Figure 7-4 gives an accurate representation of where the Doppler limitations would be for a circular flight path with a given altitude and speed. This model assumes a constant motion and gives an idea of where the coverage area will be and if guard bands should be deployed. 


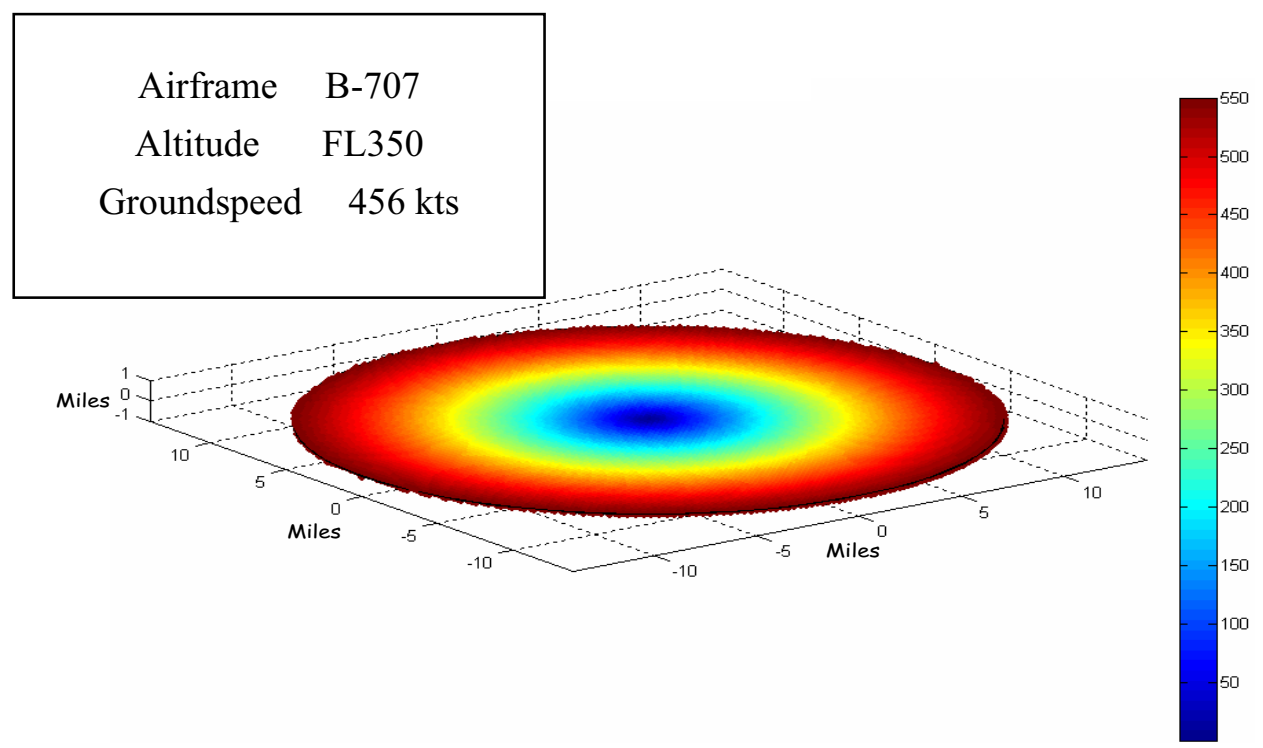

Figure 7-4: Maximum Doppler

\subsection{Multiple User Effects on Coverage}

From an RF perspective, adding multiple users can have a profound effect, known as "Cell Breathing," that limits the real or actual coverage of a cellular system. Cell breathing is more predominant in CDMA systems than in other GSM systems due to the nature of the spread spectrum technology, but will need to be considered for future 3GPP technologies that use W-CDMA, such as UMTS. Cell breathing occurs as users are added onto the sector-carrier, creating more interference on the reverse link, and thus causing the base station noise floor to rise. The interference on the reverse link is due to mobiles competing for link budget, which causes them to transmit at a higher power. This causes the coverage area to shrink, denying service to users at the edge of the cell who may have previously been in coverage because the link is less tolerant to path loss. This is known as the inhale part of the cell breathing. As the sector carrier load decreases, the base receiver sensitivity increases, allowing users at the edge of the coverage area back into service. This is the exhale part of cell breathing.

Commercial systems are planned and actually rely on cell breathing as a method to control access to the system during heavy-use periods. This is done to maintain good voice and data quality. In optimized systems, calls that are on the edge of the cell are shed to other sites that can handle the traffic and maintain good call quality. In less than optimum systems, the calls are dropped. An example is when a user that lives near a busy highway notices that between 4:00 p.m. and 6:00 p.m. there is no service available to make a call, but at other times there is service. This is possible due to rush hour traffic and the cell becoming heavily loaded. As traffic subsides, fewer users are present and the coverage area expands back to normal.

For the ACN simulation, cell breathing has been taken into account by raising the noise floor $9 \mathrm{dBm}$ over the standard base station receiver sensitivity level of $-104 \mathrm{dBm}$ [101] to $-95 \mathrm{dBm}$. This translates into a sector load of approximately $90 \%$ or 80 simultaneous clear voice calls. Note that typically $75 \%$ is the upper bound for sector loading in commercial systems. Due to the nature of the ACN system and anticipated capacity requirements, $90 \%$ is chosen because of less stringent quality of service requirements. Data or encrypted call models will decrease the sector load due to the increased reverse link power. These figures are important when determining equipment for CDMA systems since 80 calls per sector carrier indicate how many sector carriers will be needed for a given capacity requirement and path loss calculations will determine the coverage area. 


\subsection{Coverage Model Examples}

The following default values are used for the single sector and multisector coverage models in this report. Table 7-2 describes the flight characteristics and Table 7-3 describes the technology characteristics that are common to the respective technologies.

Table 7-2: Flight Characteristics

\begin{tabular}{|l|l|l|}
\hline & \multicolumn{1}{|c|}{ Single Sector } & \multicolumn{1}{c|}{ Multisector } \\
\hline Ground Speed & $200 \mathrm{kts}$ & $350 \mathrm{kts}$ \\
\hline Height & $36,000 \mathrm{ft}$ & $36,000 \mathrm{ft}$ \\
\hline Coverage & $1554 \mathrm{~km}^{2}$ & $1536 \mathrm{~km}^{2}$ \\
\hline Dimensions & $\begin{array}{l}43 \mathrm{~km} \text { diameter } \\
142 \mathrm{~km} \\
\text { circumference }\end{array}$ & $48 \mathrm{~km} \times 32 \mathrm{~km}$ \\
\hline
\end{tabular}

Table 7-3: Technology Characteristics

\begin{tabular}{|c|c|c|c|c|}
\hline & \multicolumn{3}{|c|}{ Technology } \\
\hline & & CDMA & GSM & $\mathbf{L M R}^{\mathrm{a}}$ \\
\hline \multirow{9}{*}{ 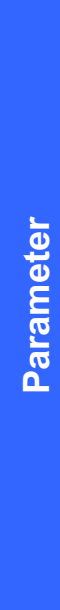 } & Base Station Transmit Power ${ }^{[1,2,3]}$ & $43 \mathrm{dBm}$ & $43 \mathrm{dBm}$ & $50 \mathrm{dBm}$ \\
\hline & Base Station Receive Sensitivity ${ }^{[1,2,3]}$ & $-102 \mathrm{dBm}$ & $-102 \mathrm{dBm}$ & $-103 \mathrm{dBm}$ \\
\hline & Base Station Transmit Frequency & $875 \mathrm{MHz}$ & $935.2 \mathrm{MHz}$ & $851 \mathrm{MHz}$ \\
\hline & Base Station Receive Frequency & $830 \mathrm{MHz}$ & $890.2 \mathrm{MHz}$ & $806 \mathrm{MHz}$ \\
\hline & Mobile Transmit Power ${ }^{[4,5]}$ & $30 \mathrm{dBm}$ & $30 \mathrm{dBm}$ & $33 \mathrm{dBm}$ \\
\hline & Mobile Receive Sensitivity ${ }^{[4,5]}$ & $-104 \mathrm{dBm}$ & $-104 \mathrm{dBm}$ & $-100 \mathrm{dBm}$ \\
\hline & Mobile Transmit Frequency & $830 \mathrm{MHz}$ & $890.2 \mathrm{MHz}$ & $806 \mathrm{MHz}$ \\
\hline & Mobile Receive Frequency & $875 \mathrm{MHz}$ & $935.2 \mathrm{MHz}$ & $851 \mathrm{MHz}$ \\
\hline & Max System Delay ${ }^{b, c}$ & $140 \mathrm{~ms}$ & $80 \mathrm{~ms}$ & $180 \mathrm{~ms}$ \\
\hline
\end{tabular}

a) LMR handheld radios are transmit limited. Vehicle mounted units usually TX up to $46 \mathrm{dBm}$.

b) Delay for GSM is with the timing advance set to a maximum of 63 bits, corresponding to $35 \mathrm{KM}$.

c) System delay for call maintainability. Delays for call quality may vary depending on the QOS required.

\subsubsection{Circular Single Sector Coverage Model}

The circular coverage model consists of a single sector and a single set of antennas (for each technology) on board the aircraft. The flight pattern and coverage area are shown in Figure 7-5. There are several issues with a circular coverage area, as discussed in previous sections, but most predominantly is the ability for station keeping of the aircraft. During a true circular route, the constant banking of the aircraft would impact the tilt of the antennas, thus having a domino affect on the coverage area and capacity of the system. 


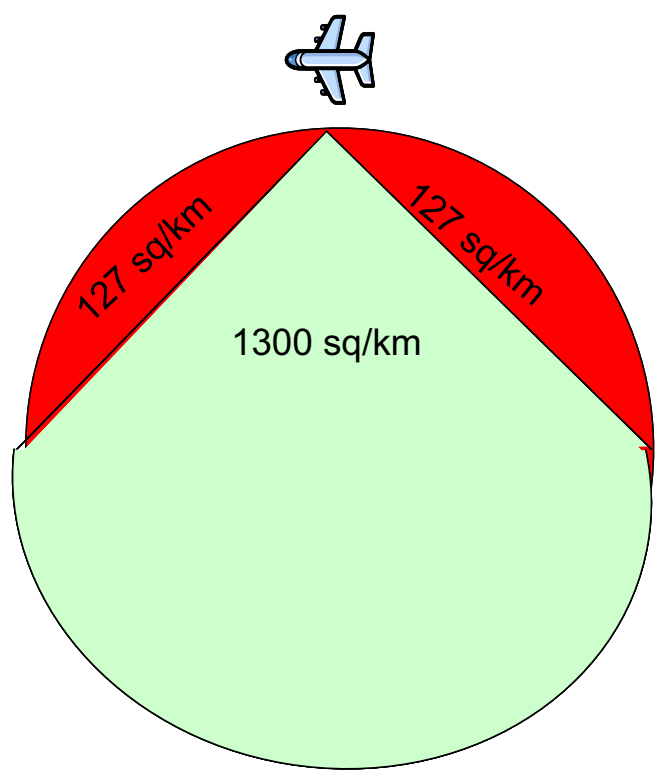

Figure 7-5: Circular Single Sector Route

\subsubsection{Benefits of the Single Sector Model}

The main benefit for the circular coverage model using a single sector system is in minimizing of the cost of equipment. Since this model is optimized for only one sector, the antenna equipment, cables, and associated weight are kept to a minimum. This model may also lend itself well to an Unmanned Area Systems (UAS) since the smaller UAS vehicles require minimum weight and power consumption to maximize station time.

Another key benefit to this configuration is the likelihood of transmitting over or receiving from outside of the intended coverage area is low. Since the coverage pattern is more predictable, interfering with the outside environment can be mitigated during the planning phases of deployment.

The final benefit of this configuration is the amount of coverage provided to the given area. As can be seen in Figure 7-6, it is possible to provide coverage to the far-reached area of operation. Granted the quality may not be ideal, but there will certainly be enough coverage to make a 911 call and relay the needed information. During the simulation, it was determined there will be approximately $17 \%$ of the coverage that will be out of service every 18 minutes, as shown in Figure 7-7. This should be considered as the baseline for all other outages in the area of operation. 


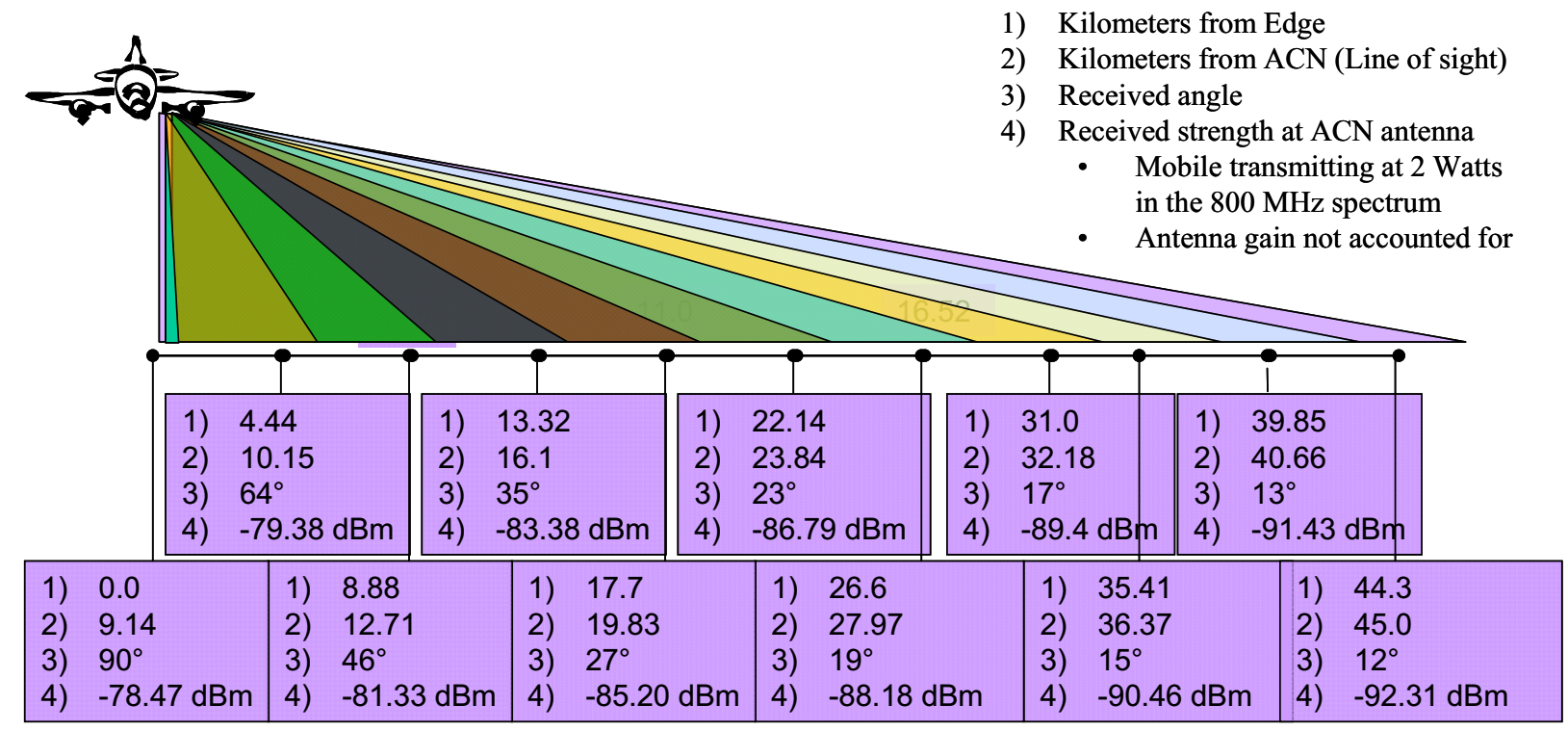

Figure 7-6: Points of Coverage
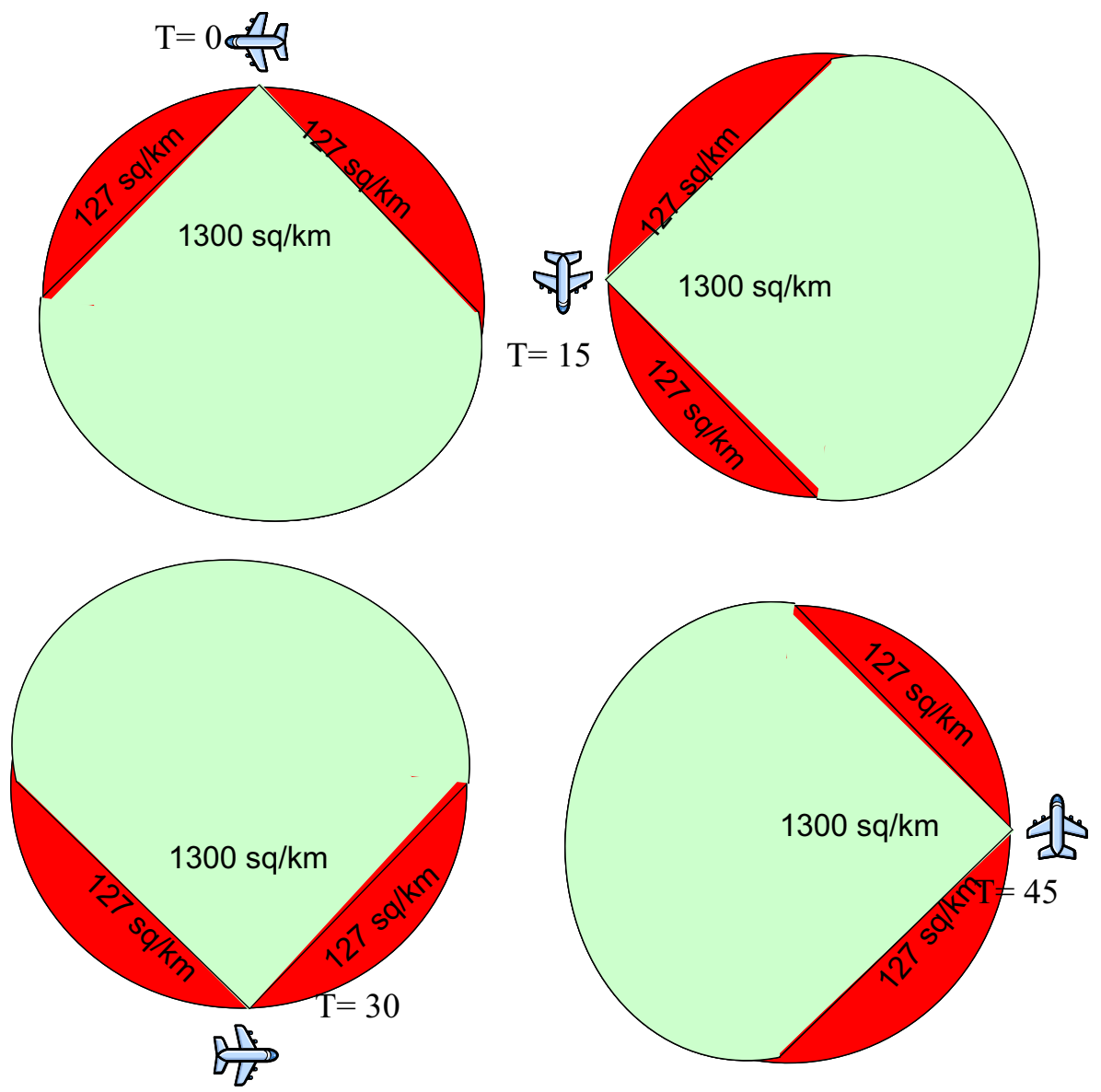

Figure 7-7: Coverage Time Lapse Example 


\subsubsection{Cons of a Single Sector Model}

The primary concern of deploying a single-sector system is the capacity of the system. Typically, cellular systems can handle close to 50 active calls per sector-carrier.[98] This is in an ideal RF environment where there is limited interference from outside sources. In the event of a natural disaster, this type of capacity constraint would hamper critical rescue efforts. The capacity constraints can be mitigated by adding additional carriers to the sector, but with additional carriers comes additional weight and power requirements of approximately $100 \mathrm{lbs}$ per carrier.

Another concern is the ability for station keeping. Maintaining a circular flight pattern over an area consistently for several hours is difficult at best. The pilot will have to contend with high winds aloft, a constant bank, and constant adjustments to maintain the circular pattern. If the aircraft strays off course slightly, there will need to be a correction that will require a bank. Once the attitude of the aircraft is changed, the coverage area will change, affecting the capability to make and receive calls. This domino effect is extremely undesirable during emergency operations.

\subsubsection{Multi Sector Coverage Model}

In the multisector coverage model, the aircraft would not be required to fly a circular route, but rather would have more options for deployment. Ideally there would be three sectors for cellular technology, all directed off one side of the aircraft. Since there are three sectors, there would have to be a minimum of three antennas per cellular technology, depending on the type of antenna used. First Responder communications, such as LMR systems, would remain with a single antenna. The simulated coverage area is shown in Figure 7-8. The shaded areas, shown in Figure 1-9, represent the optimum coverage areas for both GSM and CDMA service. Typically, the radiation pattern would be an oblong shape that would extend further out over the coverage area.

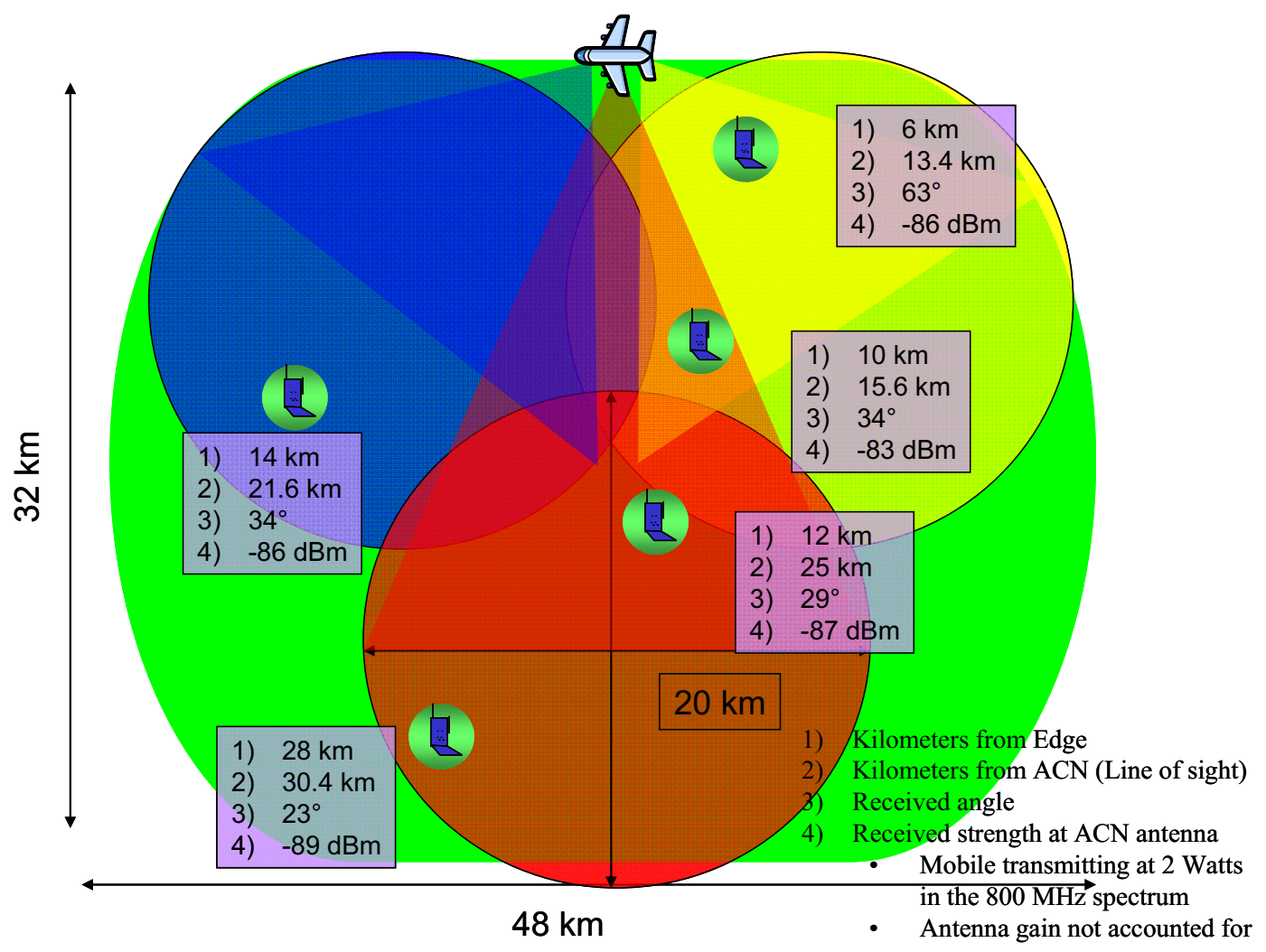

Figure 7-8: Multi-Sector Coverage Area 
Figure 7-9 is a cross section of the coverage area for the multisector model. The angles, used when calculating Doppler Effect and the distances, are used for determining both path loss and delay. While analyzing Figure 7-9, it can be determined that the edges of the middle cell will have the most amount of Doppler Effect. Additionally the edge of the coverage area will suffer from significant path loss and delay due to the line of sight distance of $38 \mathrm{~km}$. Note that these areas are out of the theoretical coverage area, and therefore the effects on the service should be negligible.

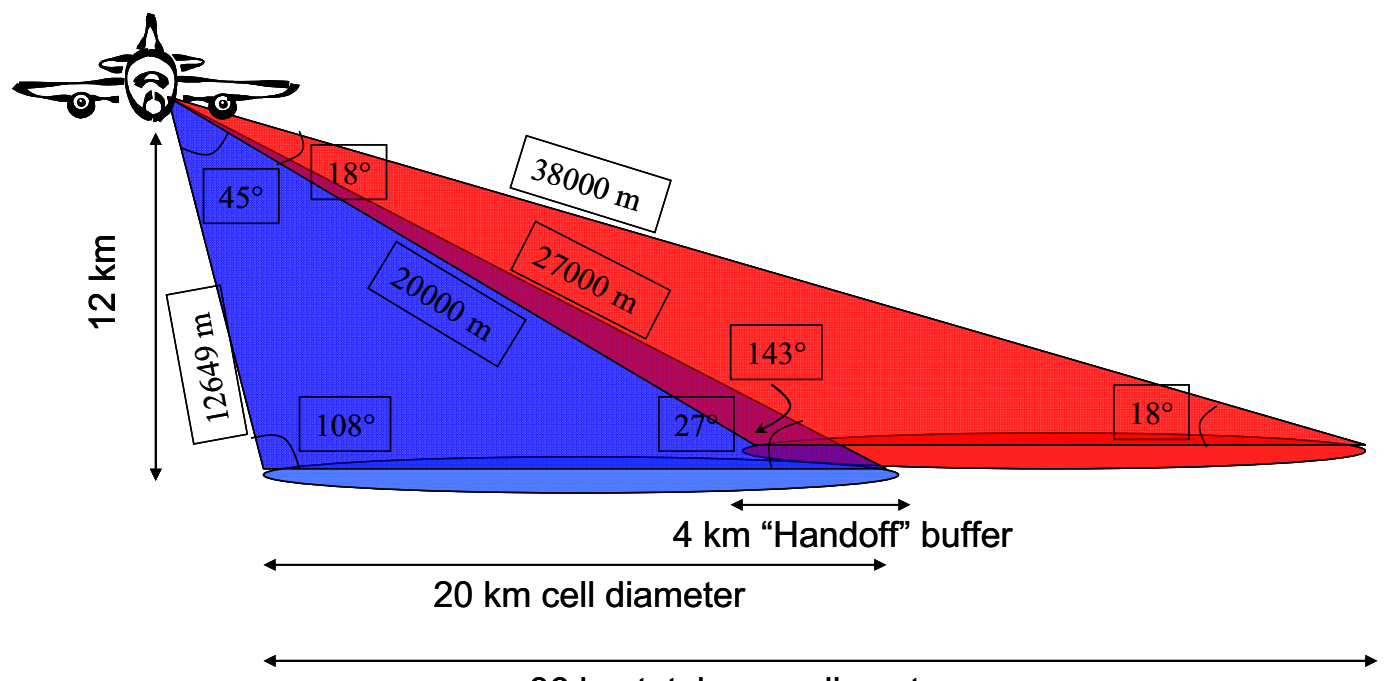

$36 \mathrm{~km}$ total cover diameter

Figure 7-9: Multisector Coverage Cross Section

\subsubsection{Benefits of Multi Sector Model}

The main benefit of having a multisector platform is the capacity gains that are realized. A three-sector system will have approximately 2.5 times more capacity than a single-sectored system. A six-sector system will have approximately 4 times the capacity of the single-sectored system.[104] In addition to the capacity improvements, there will be dramatic coverage area increases as sectors are added. Additionally, most of the current base stations have three sectors as the default configurations so there are minimal additional costs associated with this deployment.

\subsubsection{Cons of a Multi Sector Model}

The main downfall of a multisector system is the cost and weight of the additional equipment that is required. The weight alone limits the types of aircraft that can be used on the ACN. The additional weight will be in the form of antennas and cabling. The cost increases with the additional equipment will be in multiples of the number of sectors needed. For example, if three sectors are going to be deployed, the cost and weight of the antennas will have to be multiplied by three. Additionally, as more equipment is added, the less of a Quick Reaction Capability (QRC) is possible. Finally the ground user may notice dropped calls due to the handoffs that will occur. Although the technologies are designed to be robust enough to handoff in this type of application, there may be points along the coverage area that will not be able to perform handoffs.

\subsubsection{Coverage Models Using Real World Data}

In order to fully understand the relationship of speed and altitude to coverage area, five examples ranging from slow moving, low altitude light aircraft to high altitude pseudo stationary platforms. These examples use GSM limitations only since GSM technology is the most sensitive altitude and speed. All of these examples use the single sector configuration in order to show worst-case scenarios. It is interesting 
to note that as the platforms change, impacts of speed and Doppler Effect are replaced by the impacts of distance and delay. The most notable difference is between the large manned platform and the UAS.

\subsubsection{Small Aircraft (Cessna Skyhawk)}

In the first example, a Cessna Skyhawk is deployed with minimal cellular communications equipment. The Skyhawk has a cruising speed of $122 \mathrm{knots}(226 \mathrm{kp} / \mathrm{h})$ and a cruising altitude of $8000 \mathrm{ft}$.[104] Given these parameters, it can be determined that the maximum coverage area is shown in Figure 7-10.

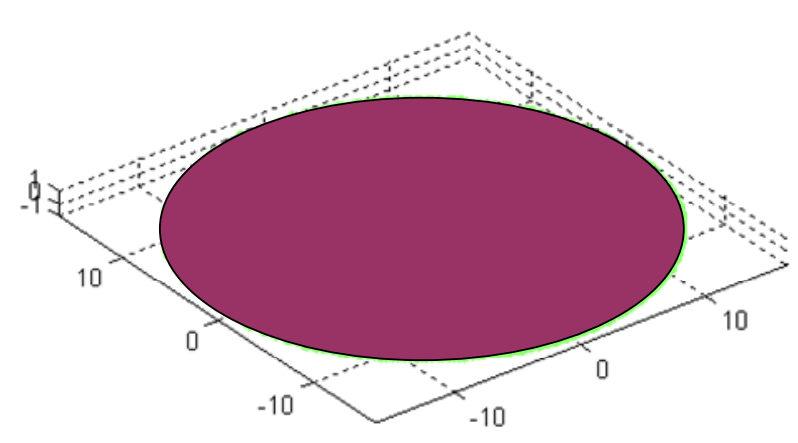

\section{Cessna Skyhawk}

$\begin{array}{lc}\text { Speed(kph) } & 226.0 \\ \text { Altitude }(\mathrm{km}) & 2.4 \\ \text { Outer Radius }(\mathrm{km}) & 17.1 \\ \text { 412Hz Radius }(\mathrm{km}) & 17.1 \\ \text { Max Doppler(Hz) } & 182.3 \\ \text { Area W/O Guard } & 918.2 \\ \quad \text { band }\left(\mathrm{km}^{\wedge} 2\right) & 918.2 \\ \text { Area W/Guard band }\left(\mathrm{km}^{\wedge} 2\right) & 1.4 \\ \text { Bank Angle }(\mathrm{deg}) & \end{array}$

Figure 7-10: Small Aircraft Coverage Model

For this type of aircraft, the typical flight time is close to 5 hours, with no aerial refueling capability. The take-off weight is $2,450 \mathrm{lbs}(1,111 \mathrm{~kg})$, and when electrical power limitations are considered, the net capacity supported by the aircraft would only be about 50 simultaneous calls.

This aircraft does not need to consider the Doppler Effect or the distance of the signal path due to the relatively low speed and altitude. The main limitation is the payload of the aircraft. Since power and weight are limited, the equipment carried on board must also be limited.

\subsubsection{Medium Aircraft (C-130)}

In the next example, a $\mathrm{C}-130$ is deployed with a full complement of cellular communications equipment. The $\mathrm{C}-130$ cruising speed is $290 \mathrm{knots}(537 \mathrm{kph})$ IAS (indicated airspeed) with a ceiling of $33,000 \mathrm{ft}(10 \mathrm{~km})$. [9] Given these parameters, the coverage area is calculated and shown in Figure 7-11. 


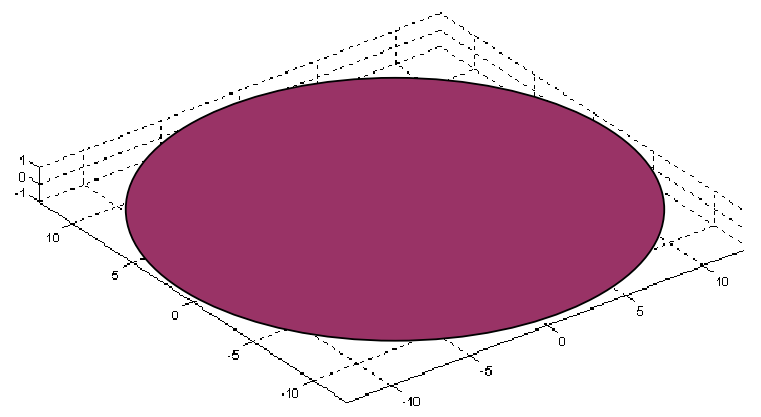

\section{C-130}

Speed(kph) $\quad 537.0$

Altitude(km) $\quad 10.1$

Outer Radius(km) $\quad 15.3$

412 Hz Radius $(\mathrm{km}) \quad 12.9$

Max Doppler $(\mathrm{Hz}) \quad 317.6$

Area W/O Guard band(km²) $\quad 730.2$

Area W/Guard band $\left(\mathrm{km}^{\wedge} 2\right)$

Bank Angle(deg)

Figure 7-11: Medium Aircraft Coverage Model

The maximum duration for this type of aircraft is 14 hours. The payload capacity is nearly $100,000 \mathrm{lb}$, which translates into essentially unlimited cellular capacity in terms of hardware deployment. The limit from capacity will be due to electrical power limitations and aircraft limitations on equipment space.

\subsubsection{Heavy Aircraft (Boeing 707)}

In the next example, the Boeing 707 is equipped similar to the $\mathrm{C}-130$. The cruising speed is $885 \mathrm{~km} / \mathrm{h}$ with an operating ceiling of 13,000 m. [10] Figure 7-12 shows the expected coverage area.

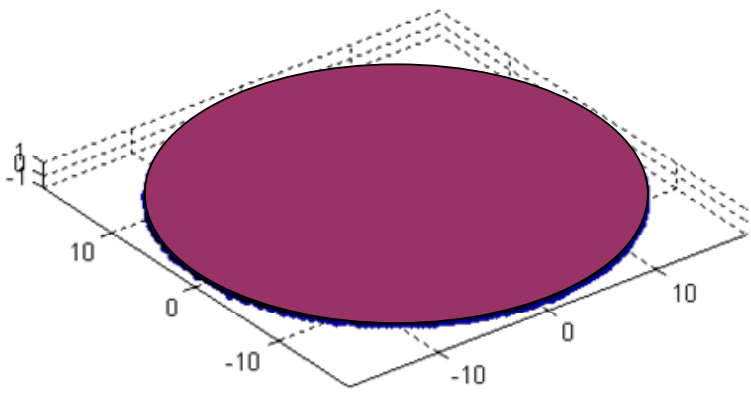

\section{Boeing 707}

Speed(kph)

885.0

Altitude $(\mathrm{km})$

Outer Radius $(\mathrm{km})$

15.0

$412 \mathrm{~Hz}$ Radius $(\mathrm{km})$

Max Doppler $(\mathrm{Hz})$

400.0

Area W/O Guard band $\left(\mathrm{km}^{\wedge} 2\right)$

706.5

Area W/Guard band $\left(\mathrm{km}^{\wedge} 2\right)$

706.5

Bank Angle(deg)

Figure 7-12: Large Aircraft Coverage Model

The relative speed and altitude of the aircraft are the contributing factors to the increased Doppler Effect. The Doppler limits are exceeded in this model, but can be mitigated by adding guard bands to the GSM system, which would increase the coverage area while decreasing capacity. 


\subsubsection{Unmanned Arial System (Predator B)}

Unmanned Area Systems (UAS) come in many_different sizes and capabilities. Predator B is used in this example due to its increased payload capacity this platform offers. The cruising speed of Predator B is $170 \mathrm{knots}(315 \mathrm{~km} / \mathrm{h})$ with a ceiling of 50,000 ft $(15.24 \mathrm{~km})$. [11] Figure 7-13 shows the expected coverage area.

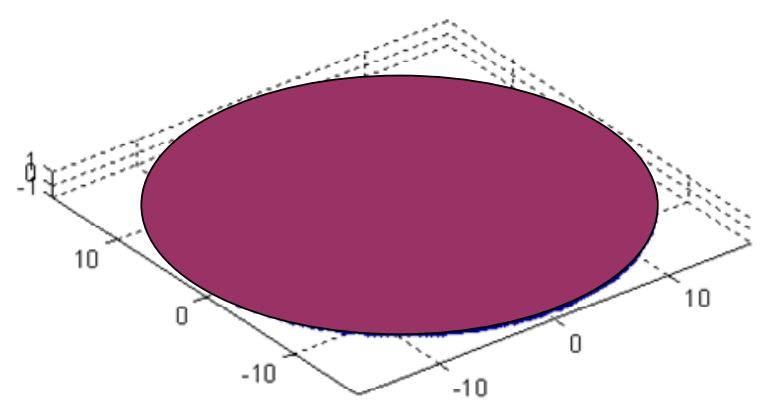

$\underline{\text { Predator } \mathrm{B}}$

Speed(kph) $\quad 315.0$

Altitude(km) 15.2

Outer Radius $(\mathrm{km})$

$412 \mathrm{~Hz}$ Radius $(\mathrm{km}) \quad 17.2$

Max Doppler $(\mathrm{Hz})$

Area W/O Guard band(km^2) 928.9

Area W/Guard band(km^2) 928.9

Bank Angle(deg) 2.6

Figure 7-13: Unmanned Aerial System Coverage Model

For the UAS, there are several different platforms to choose. The most viable solution is Predator B due to its payload capabilities. In this platform, pods could be mounted on multiple hard points giving Predator B a total payload capacity of nearly $1,500 \mathrm{lb}$.

For this platform, there is no real concern for the Doppler shift since the speed of the aircraft is relatively slow. The main concern for this platform is the altitude at which it must fly. At an altitude of $50,000 \mathrm{ft}$, the cell range of $35 \mathrm{~km}$ limits the coverage area to $1,034 \mathrm{sq} / \mathrm{km}$. If the altitude is reduced by $3,000 \mathrm{ft}$, the coverage area is increased to $1,200.71 \mathrm{sq} / \mathrm{km}$, or a coverage gain of nearly $17 \%$.

\subsubsection{High Altitude Long Endurance Lighter Than Air (HALE LTA)}

High Altitude Long Endurance Lighter than Air (HALE LTA) platforms are useful because of endurance and payload capacity. Typically, this platform will be deployed at an altitude of 75,000 feet $(23 \mathrm{~km})$. [12] Speed in this case is negligible since this is a floating platform.

Table 7-4 shows the coverage area of 1,225 sq/ $\mathrm{km}$. If a speed of 15 meters-per-second is used to account for the ground stations moving, this platform is very comparable to the UAS deployment, since distance is the limiting factor in this coverage mode. 
Table 7-4: HALE LTA Coverage Model Table

\begin{tabular}{|l|c|c|}
\hline \multicolumn{1}{|c|}{ Output } & Limit & $\begin{array}{c}\text { Calculated } \\
\text { Value }\end{array}$ \\
\hline Doppler Shift (Hz) & 412 & 31.49 \\
\hline $\begin{array}{l}\text { RSSI (Mobile) } \\
\text { (dBm) }\end{array}$ & -90 & -82.32 \\
\hline $\begin{array}{l}\text { Delay (Cell Range) } \\
\text { (km) }\end{array}$ & 35 & 35.00 \\
$\begin{array}{l}\text { Coverage Area } \\
(\mathrm{sq} / \mathrm{km})\end{array}$ & & $1,225.00$ \\
\hline
\end{tabular}

\subsection{Conclusions and Recommendations}

The final coverage area will be determined by several factors such as geographic location and population density and equipment availability. Throughout this section, the cause and effect from different variables has been discussed. The key recommendations are listed below.

- In order to reduce the Doppler Effect, keep the speed of aircraft at the minimum-allowable speed for cruising and maintain a circular flight path.

- $\quad$ Consider multiple sector systems as a possibility for deployment to provide greater coverage and capacity.

- Define the coverage area with the antenna down tilt and the vertical and horizontal beam width.

- Maximize the coverage area by reducing speed while increasing altitude to the performance envelope of the communication technology and airborne platform.

- Ensure the sector-carrier capacity will be no more than 80 users or $90 \%$ loading to maintain the coverage area. Additional users will be denied service. Adding more carriers to the same sector will increase the number of users allowed.

- $\quad$ For modeling purposes, use GSM limits to guarantee service since GSM is the least tolerant in terms of airborne coverage capabilities. 


\section{REFERENCES}

[98] QUALCOMM Deployable Base Station, Qualcomm, http:/www.qualcomm.com/qgov/products, Web page accessed 10/07

[99] TLC Solutions, Top-Level Communications Products, Integrated GSM Network (IGN), http://www.tlcconsulting.com/ign.html, Web page accessed 10/07

[100] http://www.motorola.com/governmentandenterprise/northamerica/enus/solutiondoc.aspx?navigationpath=id_803i/id_1174i\&document=learn + more $\% 2$ fid_353i, Web page accessed 7/07

[101] 3GPP TR 45.050, Version 6.2.1, Release 6, http://www.3gpp.org/ftp/Specs/html-info/45050.htm, Web page accessed $7 / 07$

[102] http://www.motorola.com/governmentandenterprise/contentdir/en_US/Files/ProductInformation/xtva_catshe et.pdf, Web page accessed 7/07

[103] Stimson, G.W. "Introduction to Airborne Radar," Hughes Aircraft Company, pp. 243-257, 1983.

[104] Rosenberg A. and Kemp S. "CDMA Capacity and Quality Optimization,” McGraw Hill, pp. 498-499, 2003.

[105] Cessna.com, "Skyhawk. Specification \& Description,” http://skyhawk.cessna.com/spec perf.chtml, Web page accessed $9 / 07$

[106] United States Coast Guard, "C-130 Hercules Specifications," http://www.uscg.mil/d11/airstationsac/hercspecs.htm, Web page accessed 9/07

[107] Boeing, “707 Specifications,” http://www.boeing.com/commercial/707family/product.html, Web page accessed 9/07

[108] General Atomics Aeronautical Systems, "Predator B," http://www.ga-asi.com/products/predator b.php, Web page accessed $9 / 07$

[109] Platforms Wireless International Corporation, http://www.plfm.net, Web page accessed 9/07 


\section{ACRONYMS}

ACN Airborne Communications Node

HALE Endurance Lighter Than Air

QRC Quick Reaction Capability

RSSI Received Signal Strength Indication

UAS Unmanned Area Systems 


\section{Airborne Platform Options}

This section defines each platform category and covers the strengths and weaknesses of each one. There are three basic categories, each having its own merits: legacy, near term and conceptual platforms. Legacy platforms use existing technologies and airframes to accomplish the mission of the Airborne Communications Node (ACN) (e.g., Boeing 707). Near term platforms are either in the final prototype stage ( $<5$ years until deployment) or newly deployed in the military community (e.g., Predator B). Conceptual platforms are purely theoretical or are in the early development stages. Generally, conceptual platforms have not been fielded for their intended mission, much less the mission of the ACN.

\subsection{Legacy Platforms}

Legacy platforms consist of platforms that are readily available. For the sake of completeness, legacy platforms encompass both air and ground assets. The greatest strength of legacy assets is that in most cases they have been proven to work and provide ample capability when requested. Secondly, the capabilities are predictable and manageable. For example, a cellular provider knows what the coverage area will be when a Cell on Wheels (COW) is deployed and can therefore plan the number of sites that are needed. The greatest weakness of legacy systems is the inflexibility that the capability brings. Again, using the COW as an example, its greatest weakness is that it cannot be deployed to areas that are inaccessible by truck; this is a highly limiting factor.

\subsubsection{COWs, COLTs and CIABs}

Cells on Wheels, Cells on Light Truck (COLT) and Cell in a Box (CIAB) are commonly used by carriers to provide extra capacity or coverage on existing networks. These platforms can either be a standalone system or a Base Transceiver Station (BTS). Stand alone systems typically use satellite backhauls with direct T1 connections into the Public Switched Telephone Network (PSTN) where BTS deployments use microwave backhaul to the Mobile Telephone Switching Office (MTSO) or Network Operations Center (NOC) Figure 8-1 depicts a typical COW, COLT and CIAB deployments.
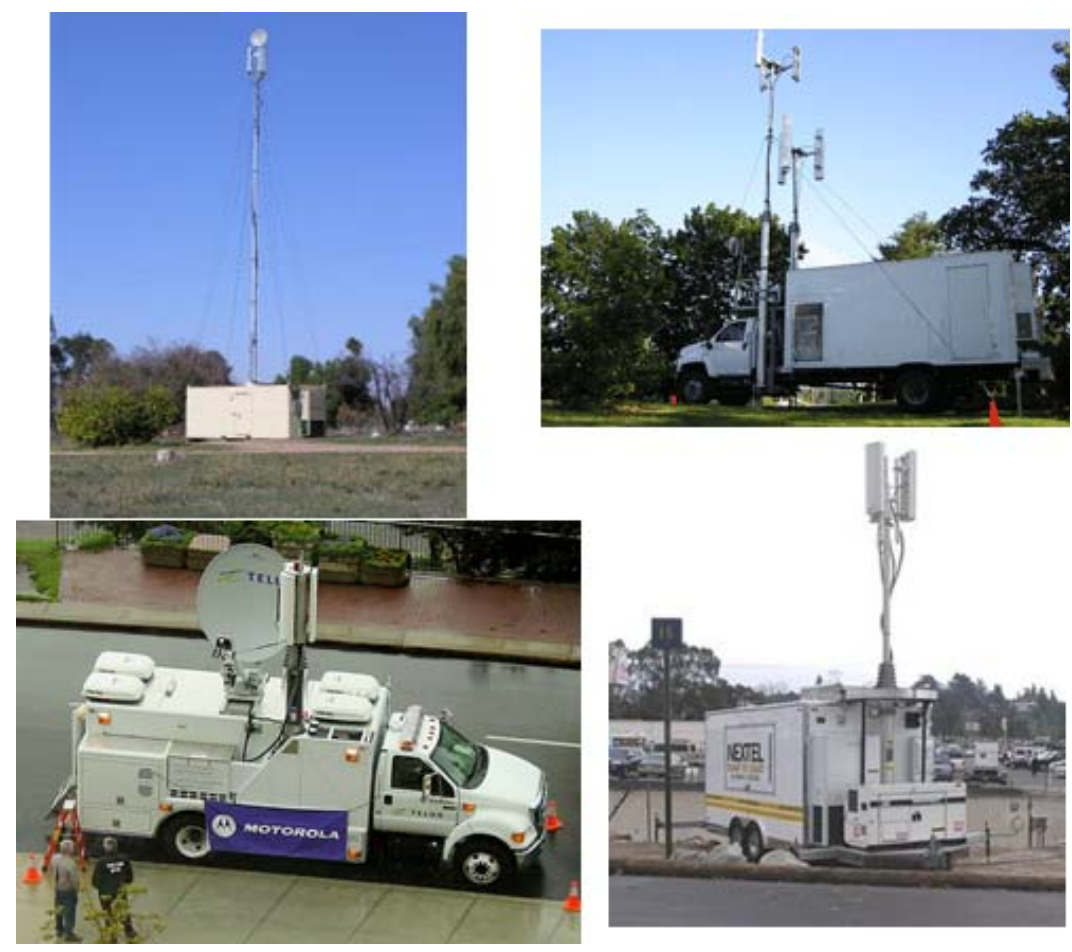

Figure 8-1: CIAB (top left), COLT (top right), satCOLT (bottom left), and COW (bottom right) 
These assets are usually towed or carried using a truck and come with a collapsible antenna mast. Power is usually provided via a generator that is brought along with the asset. On average, generators fueling this type of system will last about 48 hours. Systems of this nature were used during Katrina and most operators have COWs or COLTs to augment their existing networks in cases of natural disasters or special events to expand or enhance coverage. [110] The drawback to these systems is that they can only be deployed to areas that are accessible by the equipment towing them and refueling them. As was the case during the aftermath of Hurricane Katrina, many COWs and COLTs were unable to be deployed due to the problems presented by fuel, security and access. [111]

\subsubsection{Satellite}

Satellite networks are another option for communications during catastrophic events. They are used in emergencies as a standby communications network when existing communications networks are down. These where successfully used during Katrina but have some disadvantages: First, satellite phones require clear line of site of the sky. Urban canyons and cloudy weather conditions can cause problems with signal and quality. Second, capacity on a satellite network is significantly less than terrestrial networks. Third, the cost of using mobile satellite devices is significantly higher than using terrestrial networks. These disadvantages have led to a fourth, ubiquity. Not everyone has satellite phones and is willing to pay for them. As an example, some of those which who had satellite phones during Hurricane Katrina were not able to use them because the bills had not been paid. [111] This has lead to the majority of ordinary communications users to use cellular phones rather than satellite communications networks. Some examples of satellite phone communications systems, shown in Figure 8-2, are: Globalstar, Thuraya, and Iridium.

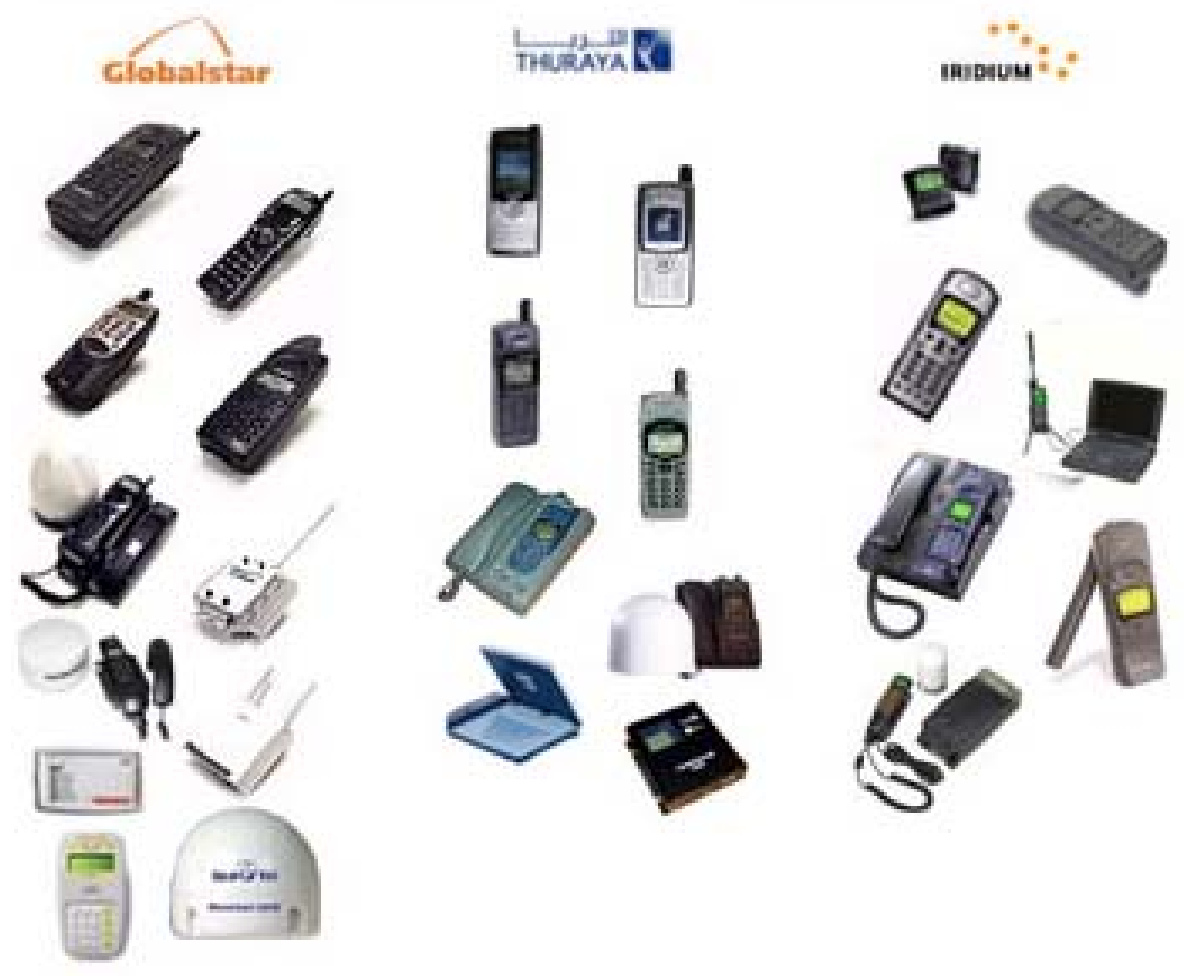

Figure 8-2: Example Satellite Devices

Satellite networks can also be used for T1 backhaul other than end-user devices. Satellite backhaul is often used for COWS in order to provide T1 backhaul to the PSTN. Using satellite in this way is often very useful when terrestrial networks are down, since it is possible to deploy a cellular system with many existing devices and use the satellite backhaul when making calls to the PSTN. Single backhauls like this 
will usually have much higher gain antennas and are not as susceptible to loss of signal as end user devices.

\subsubsection{Conventional Aircraft}

Conventional aircraft flying at 10k-30k ft are another form of legacy platform. The Doppler Effect can be a major problem with larger conventional aircraft due to the fast cruising speeds. Although, the Doppler Effect is mitigated somewhat by aircraft flying at higher altitudes, flying at higher speeds still introduces problems on not a shift in the frequency physical transmission channel but also in the Radio Link Protocol used by the cellular technologies. Aircraft flying in a circular pattern at $\sim<200$ knots can provide continuous coverage to the areas they circumscribe. Flying at faster speeds will reduce the coverage area by the aircraft. Although some of this is technology dependent, flying at a speed $<200$ knots is the lowest common denominator for cellular technologies. It is important to note that circular paths provide wider and more continuous coverage areas rather than oval or racetrack patterns. Figure 8-3 provides examples of potential platforms for communications. From left to right: Beechcraft King Air, light turboprop; Boeing 707, large turbofan; Grumman Gulfstream III, Medium Turbo Fan.
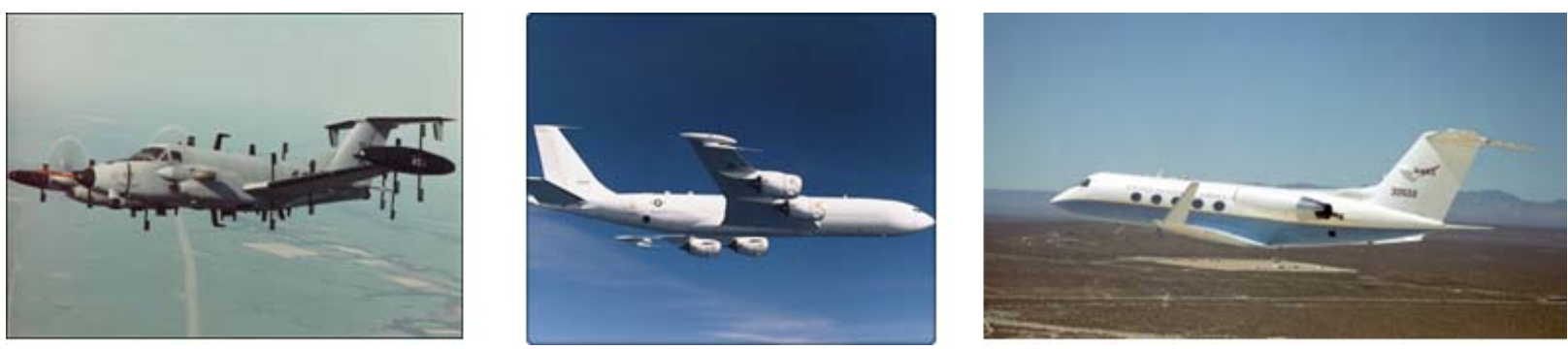

Figure 8-3: Legacy Aircraft

These aircraft can be broadly divided into two categories: large aircraft $(707,727,777$, etc.) and small aircraft (Cessna, C-12, fighter aircraft, etc.). For large aircraft, the entire wireless network is loaded onto an aircraft with the antennas directly mounted on the aircraft, or the existing antennas could be used. Power and payload capacity are considerations, but not necessarily limitations. The system can be sized to a broad range of capabilities, depending on the mission. Conversely, smaller aircraft have more limitations. The power and weight requirements tend to be more stringent, thus limiting the capabilities that can be deployed. For the smaller aircraft, the equipment may be placed in a pod that attaches to the wing or fuselage via existing hard points.

\subsection{Near Term Platforms}

Near-term platforms are platforms that either are in the late stages of development or newly deployed within the military community. The key benefit to most of these platforms is that they are made with the flexibility to perform multiple missions. For example, the Predator B, relatively new to the military community, has both the power and payload capacity to handle a small cellular system. Even though this may not have been the intended mission of the platform, the platform could accommodate this new role. The greatest weakness of these types of platforms is that they are not proven for the ACN mission or there are constraints on the missions they are allowed to participate in. Using the Predator B as an example, there are restrictions as to when and where it can fly. Although this is mainly a policy issue, it is a detractor nonetheless.

\subsubsection{Unmanned Aerial Vehicles}

Large Unmanned Aerial Vehicles (UAVs) show great potential to fit into the mission of the ACN. The Predator B is an excellent candidate since it has the power and payload capacity required. In addition to those attributes, some UAVs have the ability to sustain long durations of flight without considering 
either human factors or fueling. The key to the UAVs is the adaptability. Several systems can be deployed depending on mission requirements. Payloads for this platform would need to be custom built in order fit existing structures. Although more of policy issue, the major limitation faced by UAVs is the FAA requirements for deploying them, especially in the event of a disaster. Figure 8-4, shows an artist's depiction of the Predator B platform.

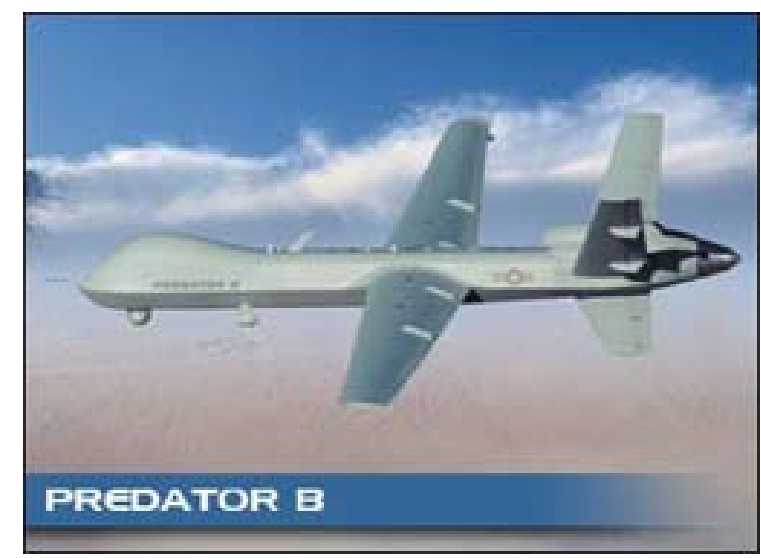

Figure 8-4: Predator B

\subsubsection{Tethered Aerostats}

Tethered aerostats use large balloons to raise antennas or equipment to altitudes of $1 \mathrm{k}-5 \mathrm{k}$ feet. Typically the tether will contain backhaul and power transmission capabilities to the equipment carried by the balloon. Commercial versions of this plaform have been deployed since 1985 to catch drug smugglers crossing the southern border of the U.S. (see Figure 8-5, right). Commercial operators have seen these platforms as opportunties for deploying emergency communications because of their proven use and long line-of-site of $\sim 200$ miles with the survenllience versions. [112] Although more susceptible to weather, they provide a longer line of site distance for communcations and continious deployment times.

Additionally, a platform of this nature could also be deployed at an Emergency Operations Center (EOC) and used for command and control and Public Safety Answering Point (PSAP) 911 call answering.

Platforms Wireless International is a vendor of a tethered aerostat communications. Platforms Wireless successfully demonstrated its airborne cellular communications system to U.S. Department of Defense and Deparment of Homeland Security on a number of separate excercises including Golden Phoenix Disaster Relief Training Exercise in California and Bold Quest military exercise at the Army's National Training Center at Fort Irwin, California. [113] (see Figure 8-5 left)
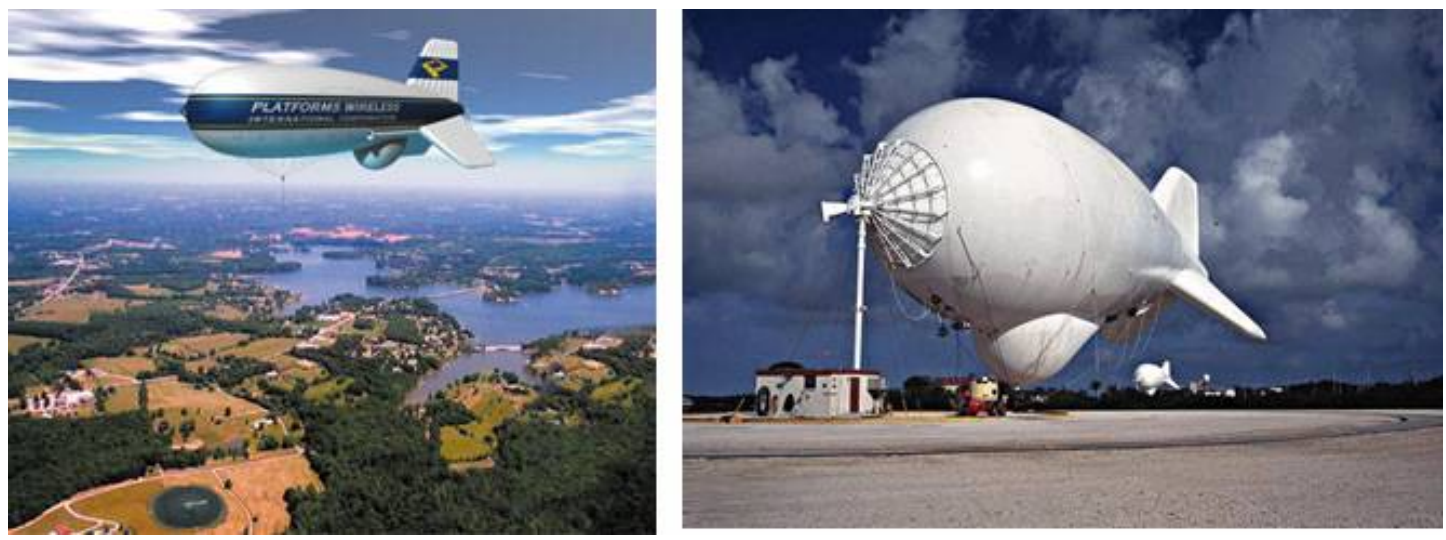

Figure 8-5: Tethered Aerostats 
TCOM and TECORE collaborated at MILCOM 2006 to demonstrate a rapidly deployable cellular system using a tethered aerostat. This solution system utilized TECORE's Rapid Response Mini-Cellular Systems (Figure 8-6, left) and TCOM's mobile 17M ${ }^{\mathrm{TM}}$ (Figure 8-6, right) Aerostat to demonstrate a quickly deployable cellular coverage for emergency response situations and tactical communications. [114]
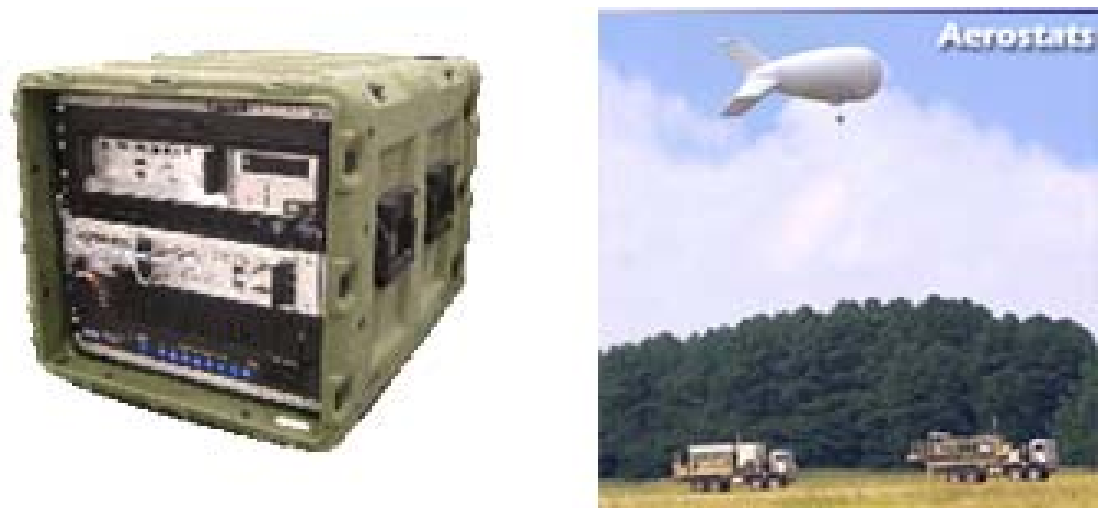

Figure 8-6: TECORE [115] and TCOM System [116]

The capability of airdropping these platforms to an affected area is a solution that has not yet been explored. Airdropping a platform of this nature could be a viable alternative since the communications equipment can still reside terrestrially while antennas and other necessary equipment go up on the balloon. Operationally, this would be easier, since space and weight constraints could be mitigated.

\subsection{Conceptual Platforms}

Although these conceptual platforms are still in development, they show promise to fulfill the ACN mission. Prototypes are giving valuable insight into the next generation war fighter capabilities. The greatest benefit of these platforms is that there is still time to tailor communications systems to a platform and have the ACN designate it as that particular platforms sole role. The key weakness of these platforms is that there is nothing available today.

\subsubsection{HALE HTA}

High Altitude Long Endurance (HALE) Heavier than Air (HTA) are examples of conceptual platforms also been under development and used for testing to provide airborne communications. Figure 8-7 shows example prototypes and platforms that have been used for testing.

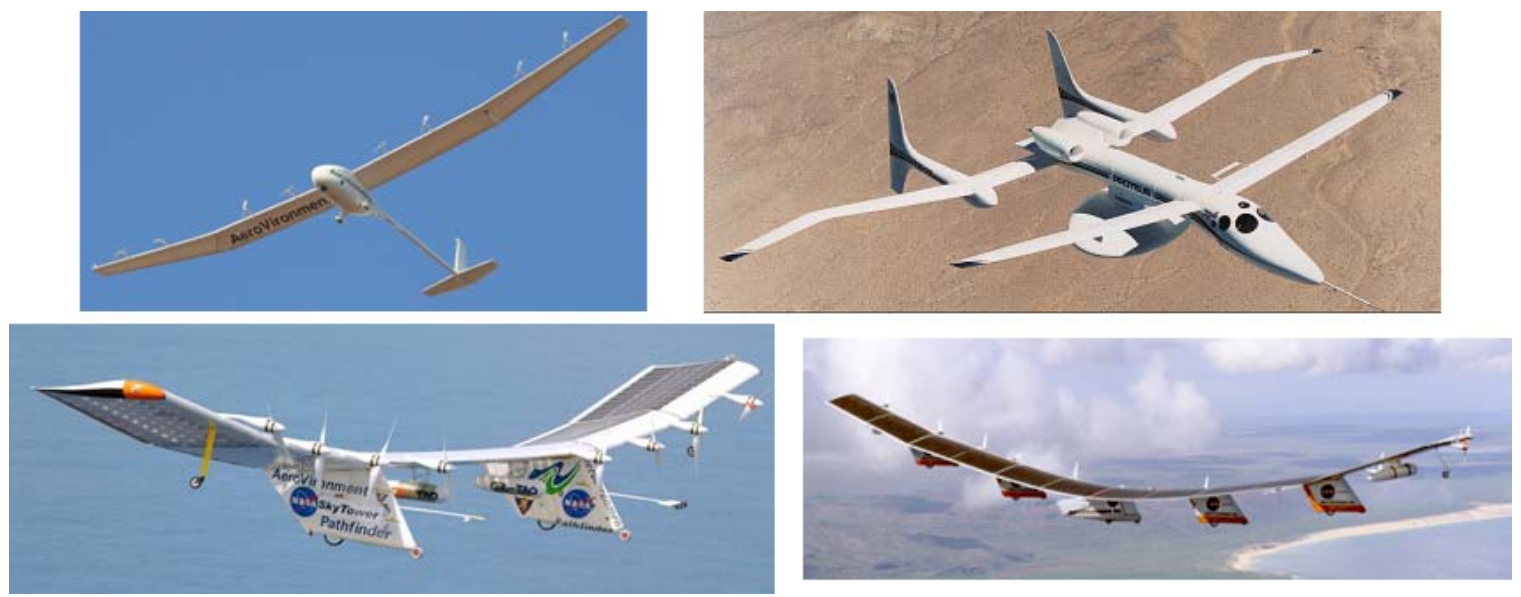

Figure 8-7: High Altitude Long Endurance Heavier than Air Platforms (HALE HTA) 
The most recent prototype, AeroVironment's Global Observer Prototype, Odyssey (Figure 8-7, top left) is projected to stay on station up to one week using fuel cells and hydrogen fuel. AeroVironment is currently working with the National Institute of Information and Communications Technology of Japan (NiCT) to deploy Global Observer to provide fixed and mobile communications. [117] Other prototypes shown in Figure 8-7: Proteus from the HALO program [118] (upper right), Pathfinder-plus solar aircraft (Lower left) and Helios (bottom right).

To date, these platforms have served as valuable test platforms for providing communications services at stratospheric altitudes. In 2004, NiCT performed an experiment using Pathfinder-plus at an altitude of $20 \mathrm{~km}$ to examine the capability of providing mobile services at this altitude. [119] The results of the experiment showed that it is possible with existing mobile devices to communicate with platforms at this altitude. This experiment provided the best data currently available in analyzing cellular communications using a high altitude platform.

\subsubsection{HALE LTA}

Research for High Altitude Long Endurance Lighter than Air (LTA) has undergone new rejuvenation in the last two years. These platforms have predicted capabilities of having long endurance times from months to years as well as payloads in the order of 1-2 tons. At high altitudes (60k-70k feet), weather can be avoided making the platform able to have survivability near to that of satellites. Air density and wind speed are also much lower at this high altitude. Unlike satellites, these platforms can be brought back to earth for repair and upgrades and returned to service. In terms of dB loss, platforms at this altitude will benefit from a $50 \mathrm{~dB}$ gain over Low Earth Orbit (LEO) satellites.[120]

NASA recently published a report examining the feasibility of using HALE LTA platforms for missile surveillance on the west and east coast of the US. [121] Using state of the art solar panels and fuel cell technology, these platforms could theoretically maintain their altitude and position for months. Currently these technologies are not advanced enough to support this type of mission. These platforms have many engineering challenges and are probably 5-10 years away from being deployed. In summary, these platforms have many benefits however they are still in the early prototype and development phases.

Many companies have taken on the challenges of advancing the development of HALE LTAs. Sanswire Networks, a subsidiary of GlobeTel, has built an airship called Stratellite ${ }^{\mathrm{TM}}$ using state of the art materials, which is currently being marketed for emergency response applications. [122] (Figure 8-8, left) STRAXX technologies are also developing a similar solution. [123] Probably the most promising effort in advancing HALE LTAs is Lockheed Martin, which was awarded a contract in January of 2006 of $\$ 149.2 \mathrm{M}$ to build and demonstrate the technical feasibility of a platform capable of doing missile defense for the U.S. [124] (Figure 8-8, middle and right).
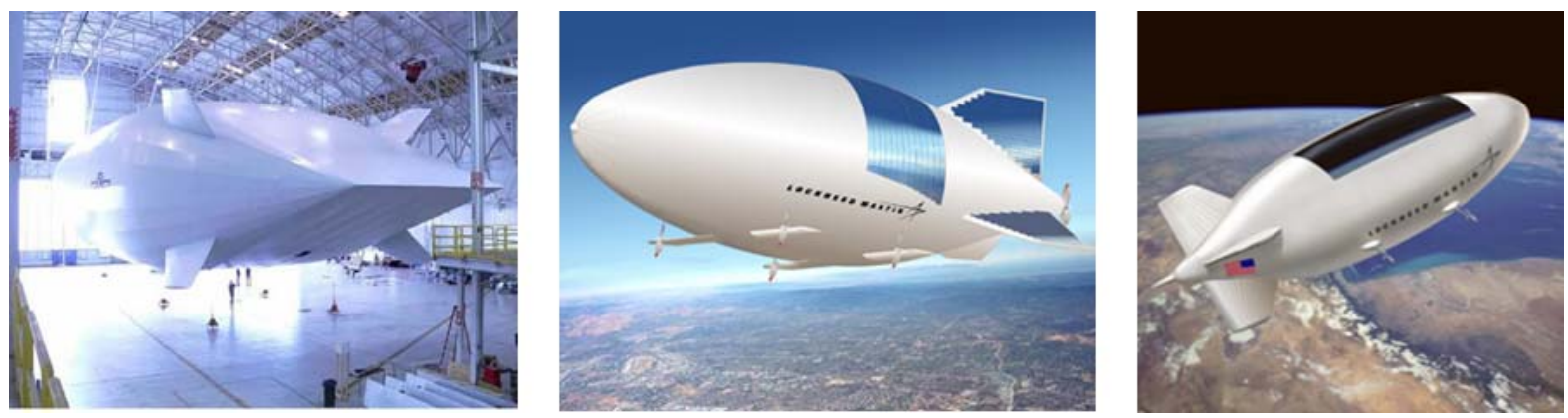

Figure 8-8: High Altitude Long Endurance Lighter than Air (HALE LTA) Platforms 


\subsection{Platform Road Map and Comparison}

Figure 8-9 shows a relative perspective for using airborne platforms as communications platforms. In the short term, conventional aircraft show the best options for providing communications system. They are currently available and rapidly deployable. Additionally, the UAV systems appear to be a good match for the near term missions of the ACN, given specific policy matters are resolved and these systems are deployed in permissible environments. Once engineering challenges for HALE LTA are overcome, these platforms would be also a potential option.

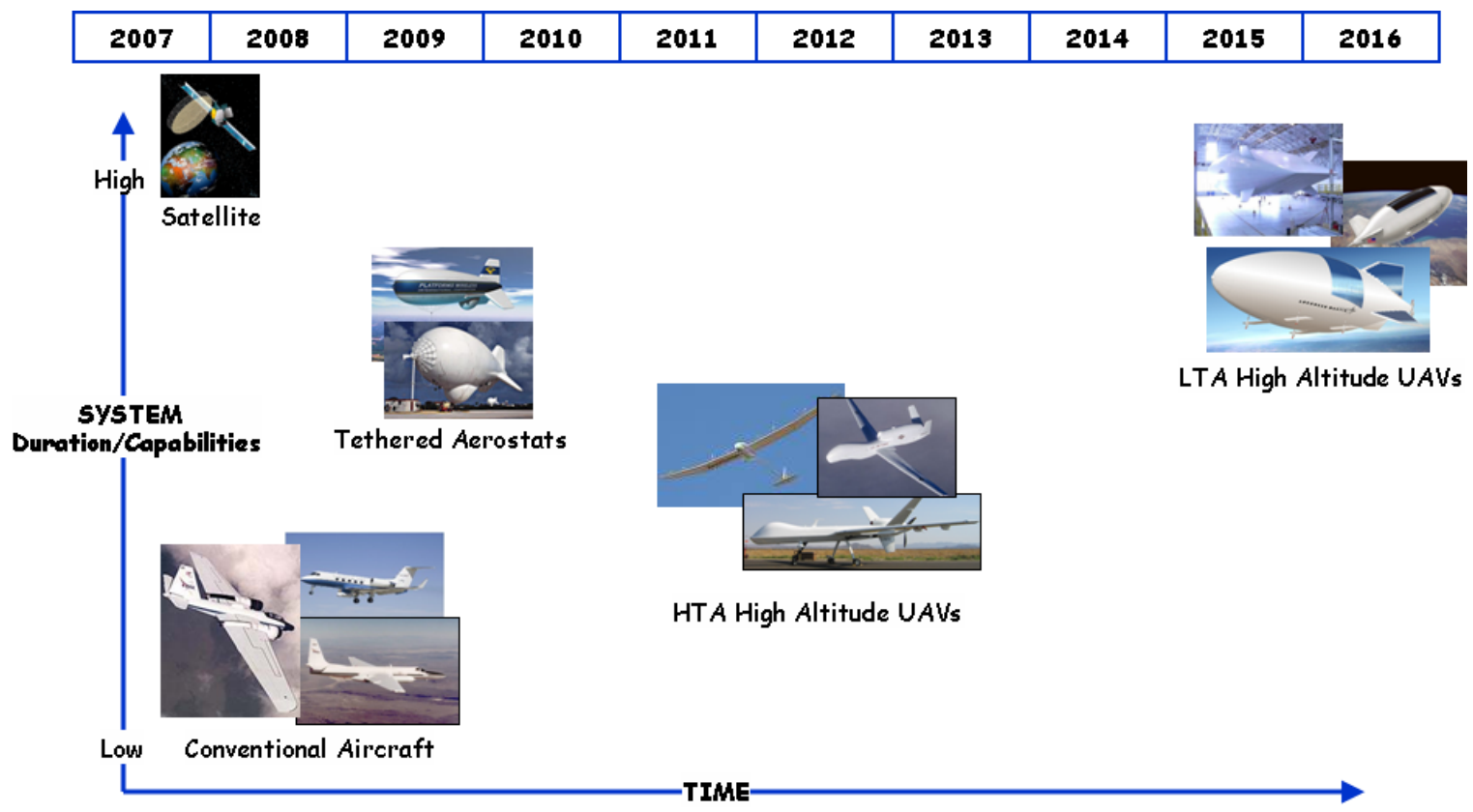

Figure 8-9: Platform Road Map

\subsection{Evaluation}

There are several platform options to consider when deploying an airborne communications node $(\mathrm{ACN})$. When considering these platforms, there are many capabilities to consider when deploying different communications capabilities on aircraft. The altitude of the aircraft determines line of site coverage which can provide communications. Available power will limit the amount of equipment and possibly the transmit power of the system. Aircraft payload is directly related to traffic capacity, based on the amount of equipment that can be brought on-board. Deployment methods for each airborne platform also varies, continuously on station aircraft can provide instantaneous communications where on station platforms will take time deploy to site. Table 8-1: Platform Comparision summarizes the strengths and weaknesses of the different platforms. 
Table 8-1: Platform Comparision

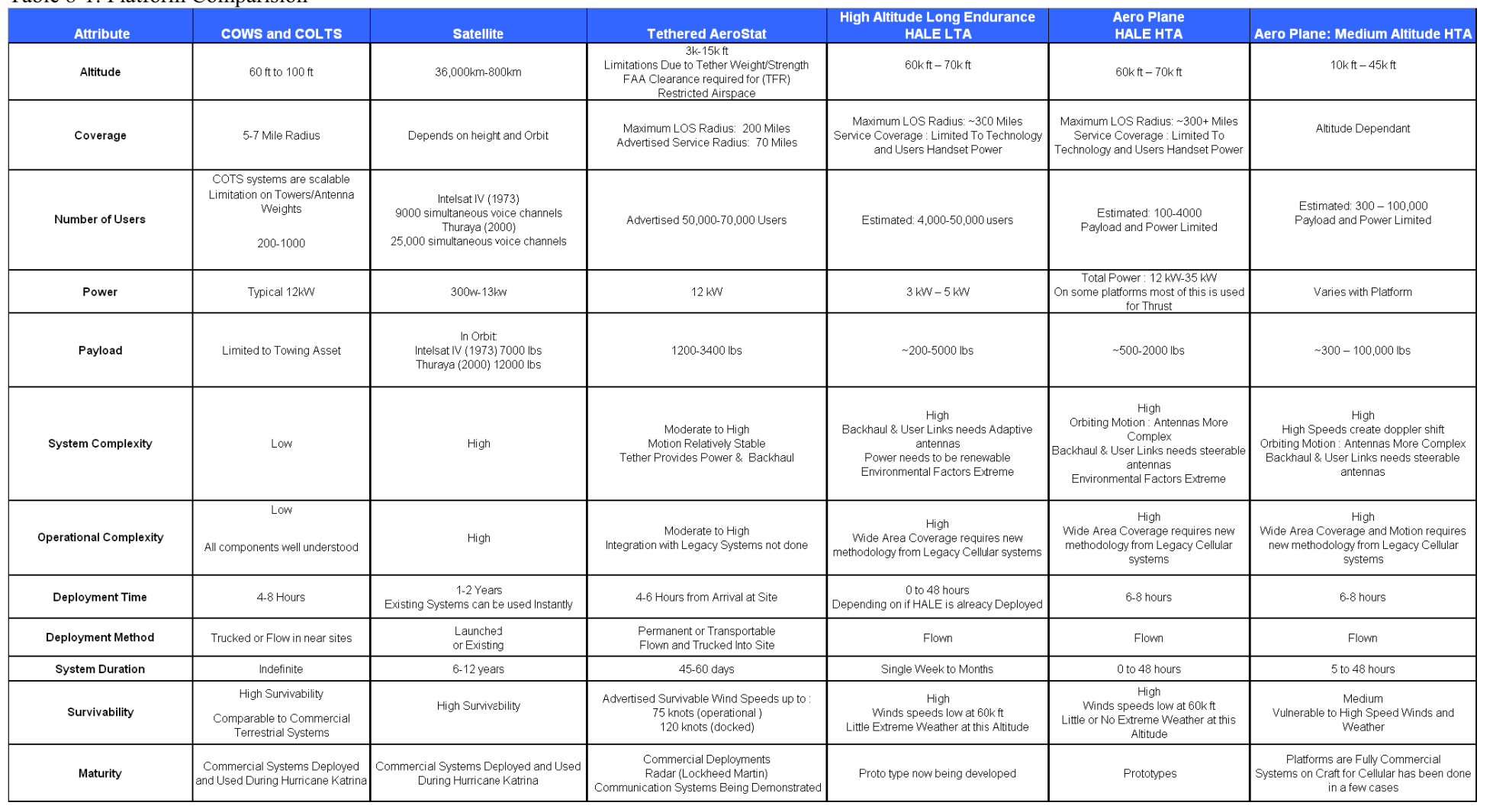




\section{REFERENCES}

[110] Chris Oberg MTS - Network Security/Network Integrity VXW NCC Rep, Personal Interview November 2006

[111] Tom Davis, Chairman A Failure of Initiative: The Final Report of the Select Bipartisan Committee to Investigate the Preparation for and Response to Hurricane Katrina, http:/www.katrina.house.gov/full katrina report.htm

[112] Tethered Aerostat Radar System (TARS) Fact Sheet, Air Force Link, http://www.af.mil/factsheets/factsheet.asp?fsID $=3507$

[113] Platforms Wireless International, see http://www.plfm.net

[114] TECORE and TCOM Unveil Rapidly Deployable Airborne Emergency Cellular Communications System at MILCOM 2006, TECORE Press Release, http://tecore.com/page/602/show_item/94/News.htm

[115] TECORE Wireless Systems Website, Rapid Response Systems, http://tecore.com/index.php?id=539

[116] TCOM Website, TCOM's 17M ${ }^{\mathrm{TM}}$ Aerostat System, http://www.tcomlp.com/17M.html

[117] Aerovironment, UAS Advanced Development Center: Projects Global Observer, http://www.avinc.com/uas dev project detail.php?id=113 http://www.avinc.com/publish/2006/07/19/985_AV_GO_SkyTower_Web_pdf

[118] COLELLA, N. J., MARTIN, J. N., and AKYILDIZ, I. F.: 'The HALO network', IEEE Commun. Mag., June 2000, pp. 142-148

[119] Masayuki Oodo, et. al, Experiments on IMT-2000 Using Unmanned Solar Powered Aircraft at an Altitude of 20 km, IEEE Transactions On Vehicular Technology, VOL. 54, NO. 4, JULY 2005

[120] DJUKNIC, G. M., FREIDENFELDS, J., and OKUNEV, Y.: 'Establishing wireless communications services via high altitude aeronautical platforms: a concept whose time has come?', IEEE Commun. Mag., September 1997, pp. 128-135

[121] COLOZZA A. and DOLCE, J. L.: 'High Altitude, Long Endurance Airships for Coastal Surveillance,' NASA/TM-2005-213427 http://gltrs.grc.nasa.gov

[122] Sanswire Networks, see http://www.sanswire.com/index.htm

[123] Straxx Near Space Technology, http://www.stratxx.com/technology.html

[124] Lockheed Martin HAA platform, http://www.lockheedmartin.com/wms/findPage.do?dsp=fec\&ci=14477 


\title{
ACRONYMS
}

\author{
ACN Airborne Communications Node \\ BTS Base Transceiver Station \\ CIAB Cell in a Box \\ COLT Cells on Light Truck \\ COW Cell on Wheels \\ EOC Emergency Operations Center \\ HALE High Altitude Long Endurance \\ HTA Heavier than Air \\ LEO Low Earth Orbit \\ LTA Lighter than Air \\ MTSO Mobile Telephone Switching Office \\ NiCT National Institute of Information and Communications Technology of Japan \\ NOC Network Operations Center \\ PSAP Public Safety Answering Point \\ PSTN Public Switched Telephone Network \\ TARS Tethered Aerostat Radar System \\ UAV Unmanned Aerial Vehicle
}




\section{Prior Art}

\subsection{Introduction}

The purpose of this section is to provide a summarized description of existing commercial applications, experiments, research, and patents of airborne communications as uncovered in the research of this report.

\subsection{Commercial Airborne Cellular Systems}

After Hurricane Katrina, varieties of airborne emergency communication systems have been emerging. These systems come in a variety of different platforms and vendors. These systems have been prototyped and are available for purchase from the respective vendors.

\subsubsection{AeroFon}

AeroFon offers a range of airborne wireless communications products available for purchase. They are currently marketing two different product lines: Aero Communications System (ACS) and AeroRed. From their Web site:

"ACS is designed to provide $24 \times 7$ cellular communications coverage. The aerial vehicle is an aircraft selected for cost, reliability, and performance. Proprietary modifications allow operation in the stratosphere, above airline traffic, and weather. Employing proprietary logistics systems, ACS reliability exceeds North American cellular standards (ninety percent of coverage area, ninety percent of the time). Ideal applications would be for operators providing coverage in un-served "fixed" areas, or by governments for supporting disfavored social groups. AeroFon's ACS has withstood rigorous "Technical Due Diligence" from Vivo in Brazil, South America's largest CDMA cellular operator.

AeroRed can provide temporary coverage for special events and for natural and man-made disasters. The deployment of an AeroRed system would be appropriate for inter-communications between the emergency response services after hurricanes, during power outages, floods, earthquakes, volcanic eruptions, and terrorist actions. A complete telecommunications systems infrastructure is located aboard a light aircraft ready for action at a moments notice. With pre-deployment of subscriber units, system operation commences immediately upon the AeroRed system arriving on station above the coverage area. Comprehensive interconnection via satellite or terrestrial microwave radio links to other networks is also possible."

In a report released by Qualcomm and AeroFon on May 29, 2003, coverage and test call data was shown in the San Diego, California area.[138] This data is shown in Figure 9-1. This experiment used a Qualcomm Deployable Base Station (QDBS) platform on a Cessna 421C Golden Eagle. One unique aspect of this technique is the implementation of a separate ground station, although the system has options for satellite backhaul as well. This vendor offers a turnkey solution providing pilots all equipment and management of the system to the provider. It is not known if AeroFon has a licensing arrangement with U.S. Cellular Carriers. This could provide a key role in their integration with existing systems. Based on the details of this experiment it is believed that this experiment could support $\sim 50$ simultaneous Code for Division Multiple Access (CDMA) calls in ideal conditions. 


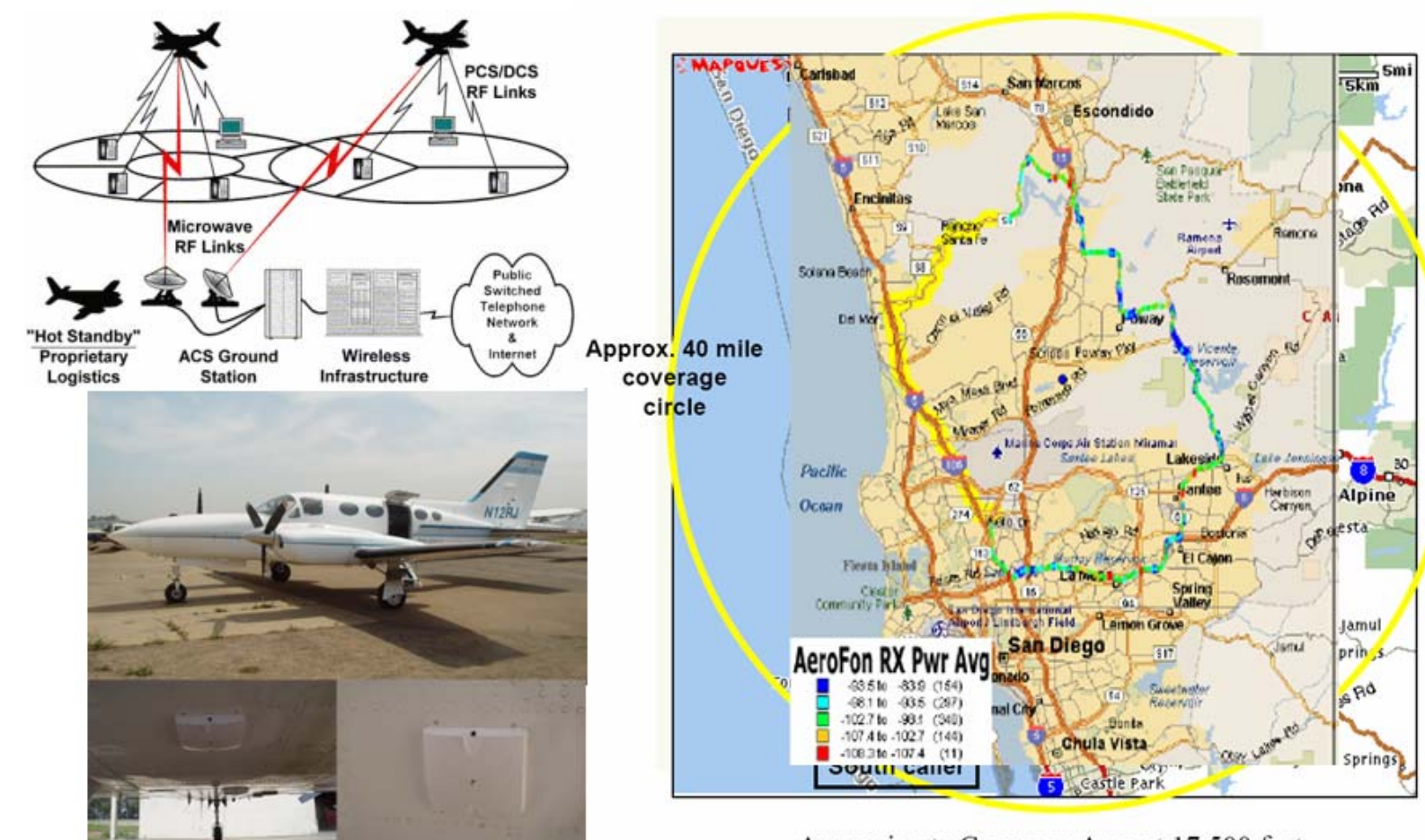

Approximate Coverage Area at 17,500 feet

Figure 9-1: AeroFon's Experiment [138]

AeroFon also has also demonstrated the capability to provide in-band repeating using an audio gateway. They are marketing this capability on their AeroRed platform. [139]

\subsubsection{Rapidly Deployable Airborne Emergency Cellular Communications System}

TCOM and TECORE collaborated at MILCOM 2006 to demonstrate a rapidly deployable cellular system using a tethered aerostat. [140] This Global System for Mobile (GSM)/CDMA wireless system utilized TECORE's Rapid Response Mini-Cellular Systems (Figure 9-2, left) and TCOM's mobile 17M ${ }^{\mathrm{TM}}$ (Figure 9-2, right) Aerostat to demonstrate a quickly deployable cellular coverage for emergency response situations and tactical communications. TECORE's Rapid Response Mini-Cellular Systems offers a combination GSM and CDMA capabilities in a single box, which is unique to TECORE.
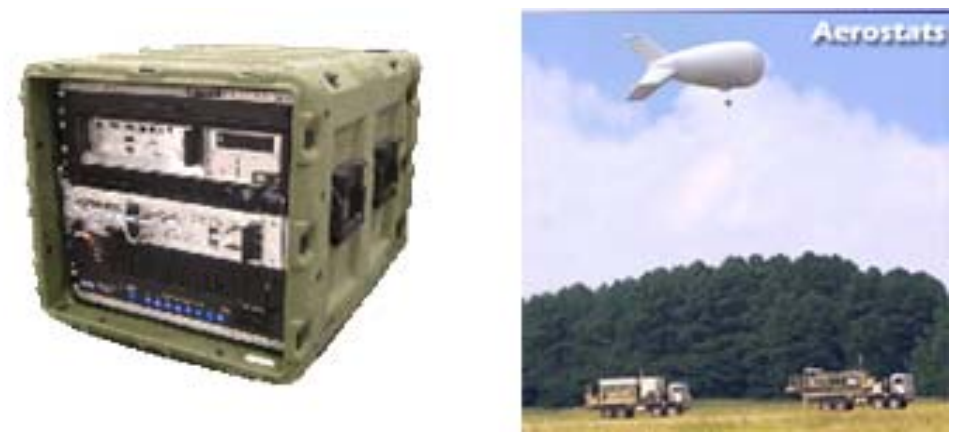

Figure 9-2: TECORE [141] and TCOM System [142]

The $17 \mathrm{M}^{\mathrm{TM}}$ solution supports a $200 \mathrm{lb}$ payload and TECORE Rapid Response Mini-Cellular Systems in this configuration, and will most likely support $\sim 50$ simultaneous CDMA users in a pure CDMA configuration and a comparable amount of users for a GSM configuration. 


\subsubsection{Platforms Wireless}

Platforms Wireless International provides airborne telecommunication solutions to augment, replace, and/or supplement conventional terrestrial and satellite systems by means of a tethered aerostat platform. This aerostat system has the capability of delivering virtually any form of communications desired. Platforms Wireless International has demonstrated this capability using cellular platform that they have developed for the system. Essentially, this is placing a large-scale base station or Base Transceiver Station (BTS) inside the blimp. The tether, which holds the blimp pseudo-stationary, also contains a fiber optic cable and power for the BTS.

Platforms Wireless International claims the following capabilities of the platform. The blimp is capable of 3,000-15,000 ft of flight and can support 50,000-80,000 wireless users (not simultaneous calls). [143] Figure 9-3 illustrates the coverage area and architecture. Additionally, it is capable of sustaining winds of up to 75 knots and surviving winds of up to 90 knots. When the station is moored, it is capable of winds of up to 120 knots. The station can be deployed continuously for 45-60 days before requiring routine maintenance. [144]

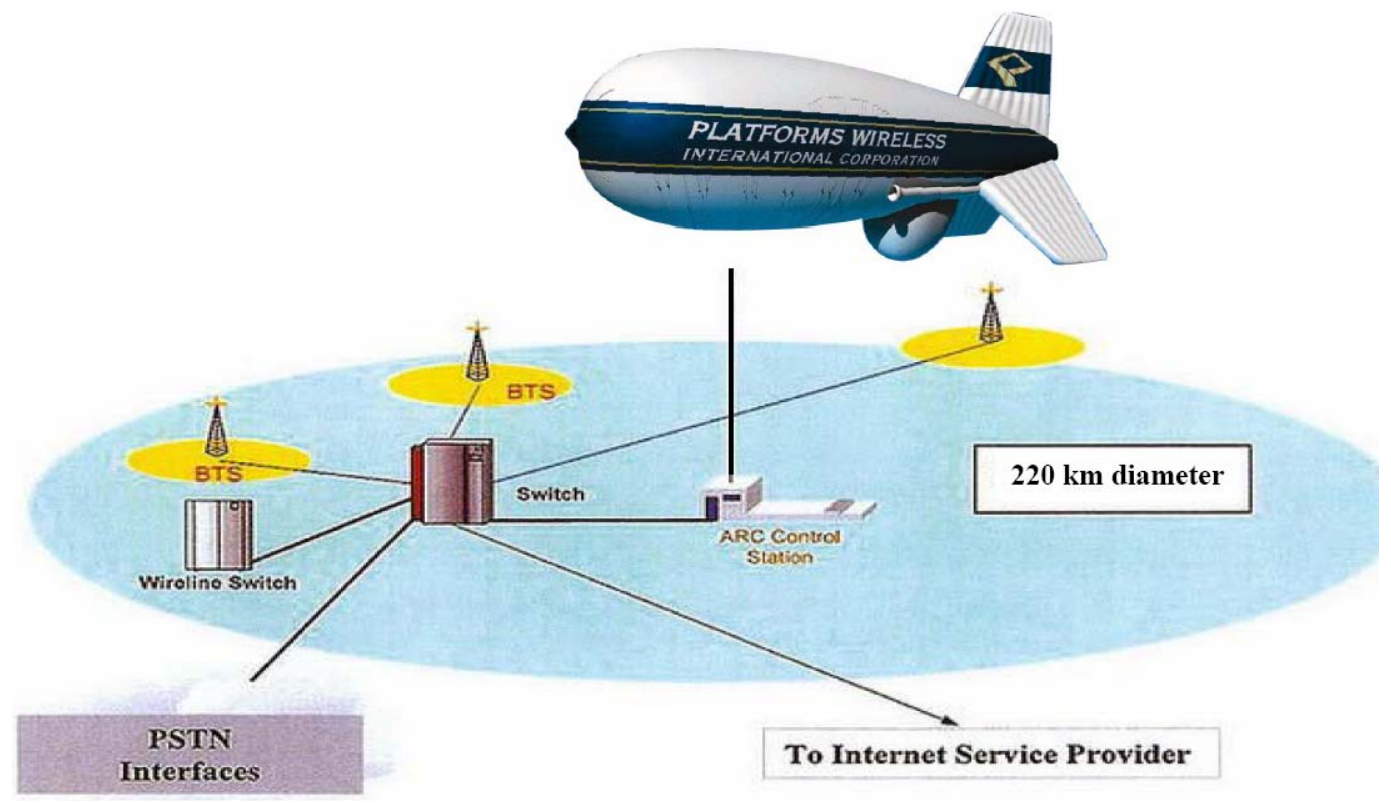

Figure 9-3: Platforms Wireless Solution [143]

On July 30, 2007, Platforms Wireless International announced its first sale of its ROSETTA-911 system. The customer of the system has not been announced.[145] However, INL is aware that Platforms Wireless International did do a demonstration for the Department of Defense and Department of Homeland Security this summer at Ft. Huachuca, Arizona. They had received funding from both organizations to do this demonstration.

\subsection{Existing Emergency Systems}

To avoid the disparity between communications networks it is important to understand what current communications exist so that their capabilities could be leveraged to for any type of ACN platform. Two primary emergency capabilities are important to consider for integration with the ACN platform: the Rivada Deployable Networks and Joint Incident Site Communications Capability.

\subsubsection{Rivada Deployable Networks}

Currently there are many different emergency response communications systems that are deployed with many different agencies. In terms of federal assets, Federal Emergency Management Agency (FEMA) and U.S. Northern Command (NORTHCOM) both have Rivada Deployable Networks (RDN). RDNs support CDMA cellular calls, interoperable radio with satellite reach back into a Public-Switched Telephone Network (PSTN) and 
Internet. Some Air National Guard units also have these networks. Figure 9-4 illustrates an example of the RDN. The RDN owner, as well as its deployed location in the U.S., is shown in Figure 9-5.

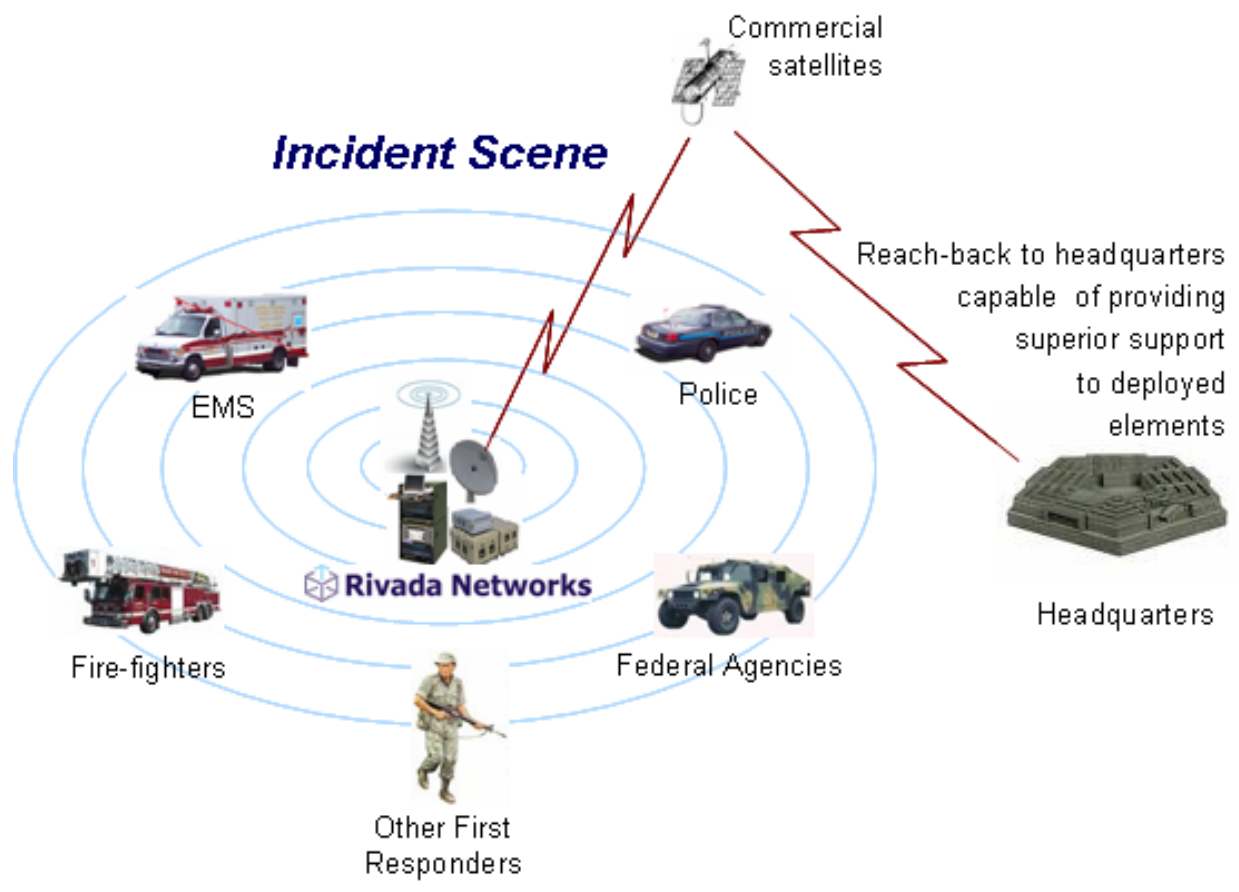

Figure 9-4: Rivada Deployable Network

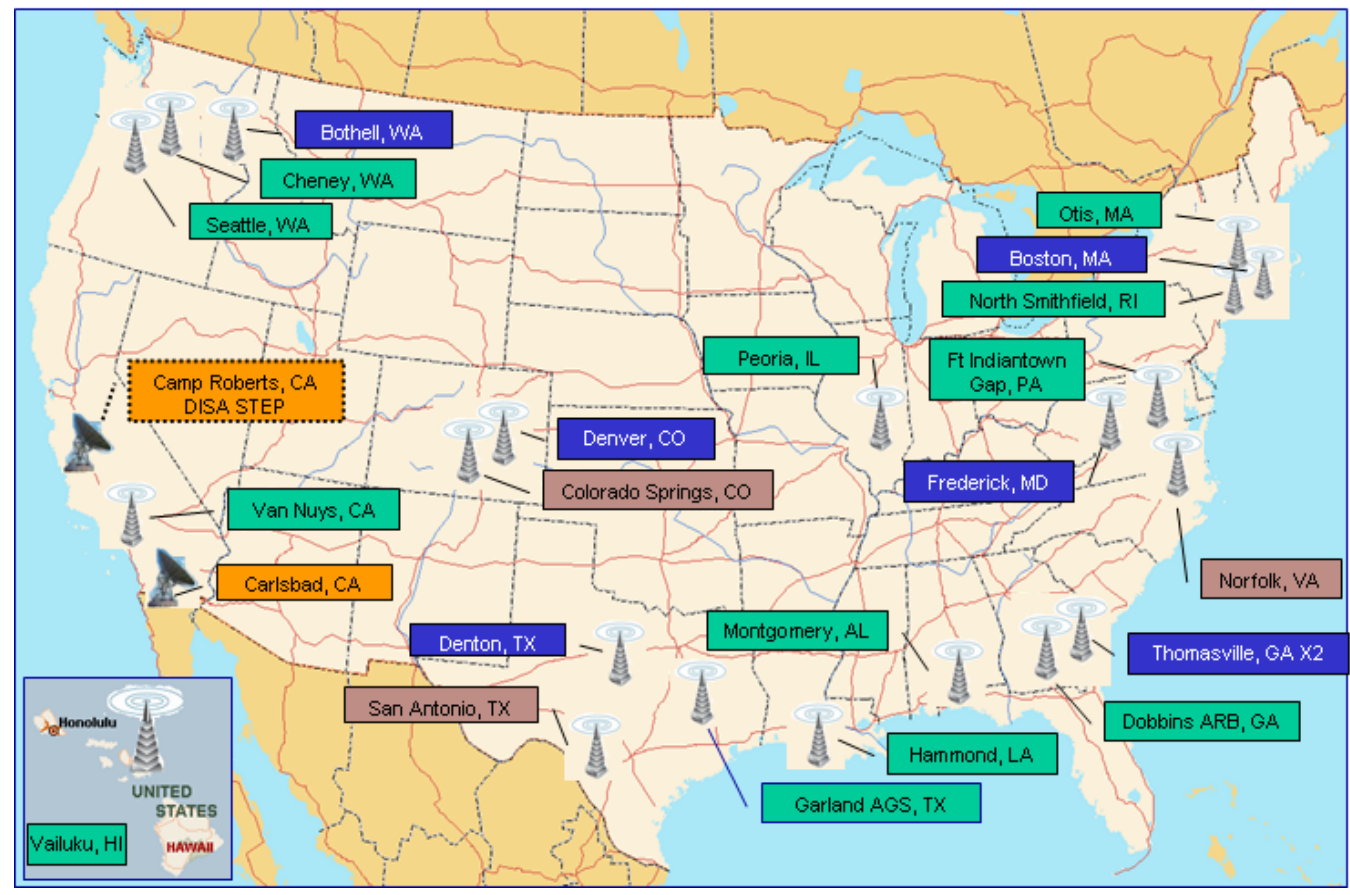

Legend

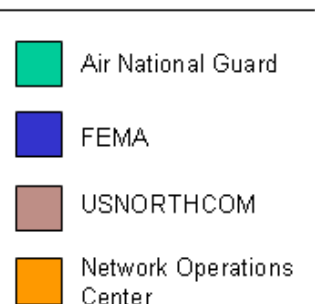

Figure 9-5: Rivada Deployable Networks - U.S.

Additionally Rivada is beginning to build FEMA, NORTHCOM, and Air National Guard systems to be interoperable with one another. In other words, cell phone users on a FEMA network will be able to roam onto the other two networks. For all networks, it is not necessary to hand out phones during an emergency since the RDNs all support users everyday use cell phone to roam onto the RDN. This is all done by using a centrally managed Home Location Register (HLR), which Rivada maintains in Camp Roberts, California. 


\subsubsection{Joint Incident Site Communications Capability}

National Guard units across the country are beginning to deploy a capability known as Joint Incident Site Communications Capability (JISCC). The JISCC provides incident site radio communications by using a UHF repeater and base station and UHF radios along with an interoperable radio platform. In addition to radio communications, it also has the VTC, local area network (LAN), and wired and wireless Internet Protocol (IP) telephones. Reach back is provided via a satellite antenna system. A pictorial overview of the JISCC capabilities is shown in Figure 9-6.

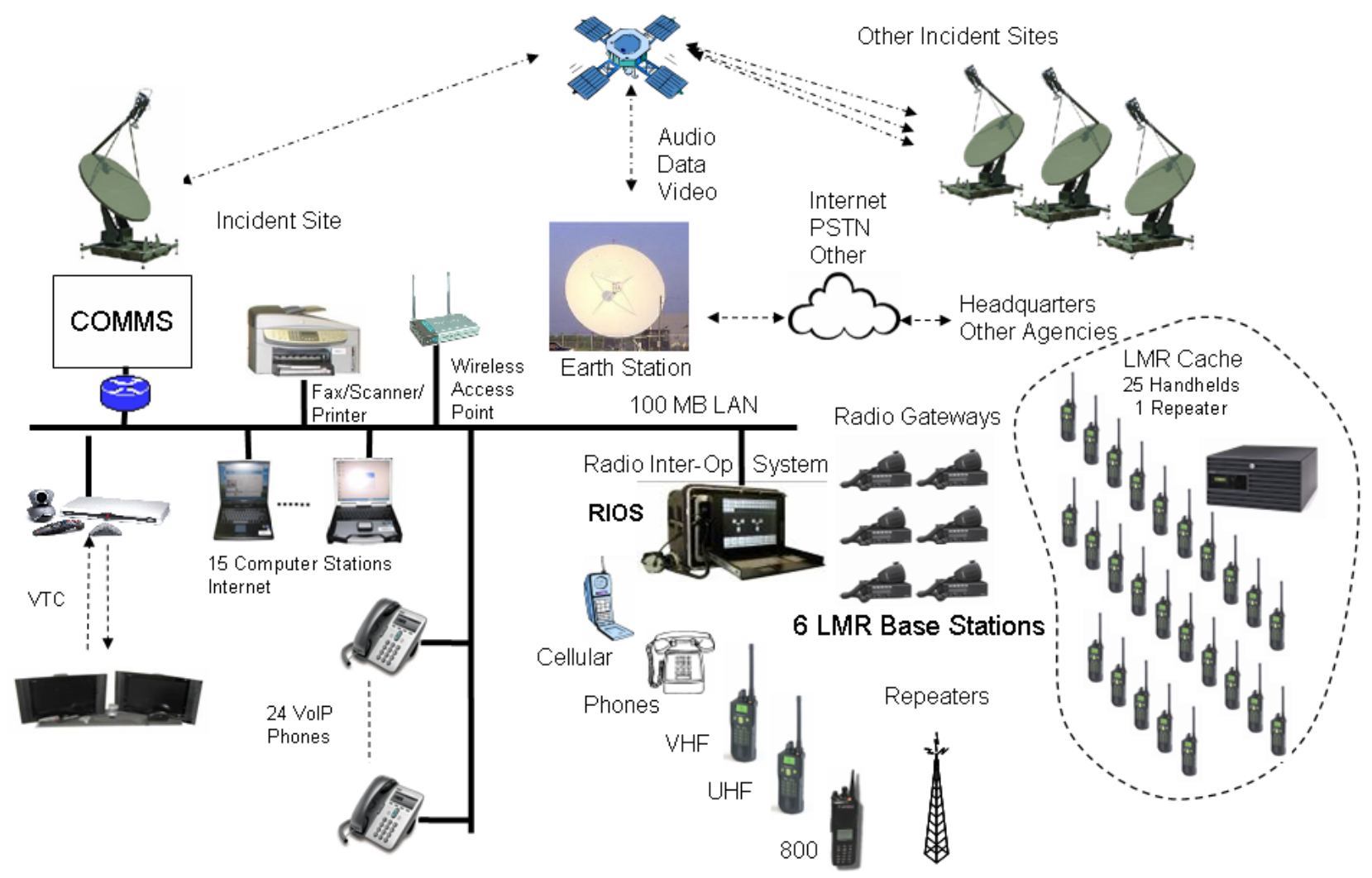

Figure 9-6: JISSC Capability Overview

\subsection{Emerging Solutions}

Emerging solutions are concepts that have not reached a beta phase or prototype phase. These solutions presented in this section are conceptual or in the design and experimentation phases of development.

\subsubsection{SkyTower}

AeroVironment Inc. (AV), an Unmanned Aerial Vehicle (UAV) manufacturer, is currently working in cooperation with the National Institute of Information and Communications Technology (NiCT) of Japan to build an airborne communications called SkyTower. AV is developing a persistent stratospheric platform, called Global Observer (GO), for the SkyTower project. GO would be capable of a 1-week flight duration and at $65 \mathrm{k}$ ft. Payloads will initial support $400 \mathrm{lbs}$; however, the GO-2 is being designed for a 1,000 lb payload. The AV concept is captured below in Figure 9-7. 


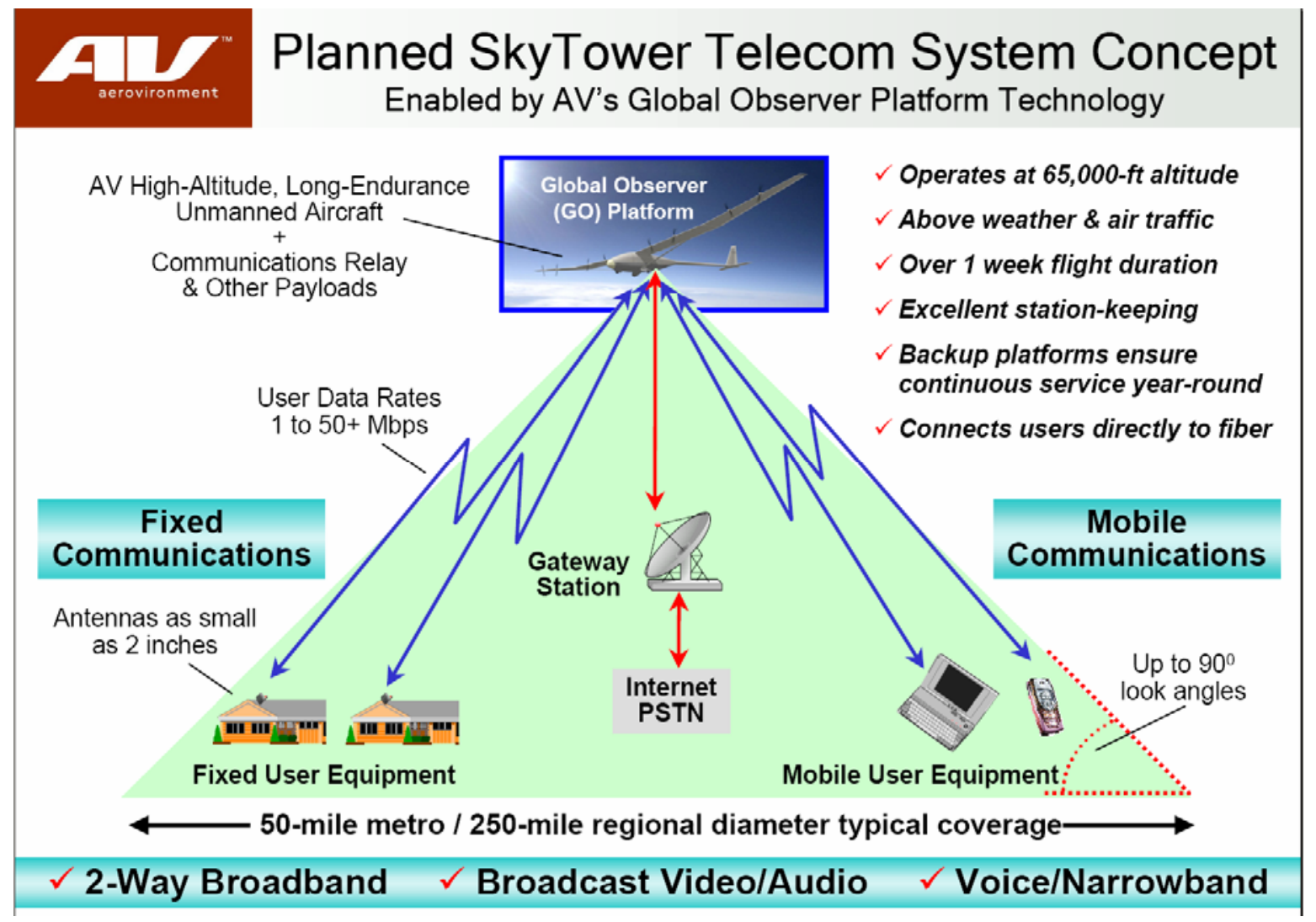

Figure 9-7: AV SkyTower Concept

\subsubsection{Sanswire Stratellite ${ }^{\mathrm{TM}}$}

Sanswire is another company developing a state of the art stratospheric airship called Stratellite ${ }^{\mathrm{TM}}$. This airship is designed for many different applications, including telecommunications. The aircraft is designed to go to $65 \mathrm{k}-70 \mathrm{k}$ feet and stay on station for months at a time. Its payload is estimated to be 2 tons. Figure $9-8$ shows a picture of the Stratellite ${ }^{\mathrm{TM}}$ and the telecom concept.[150]

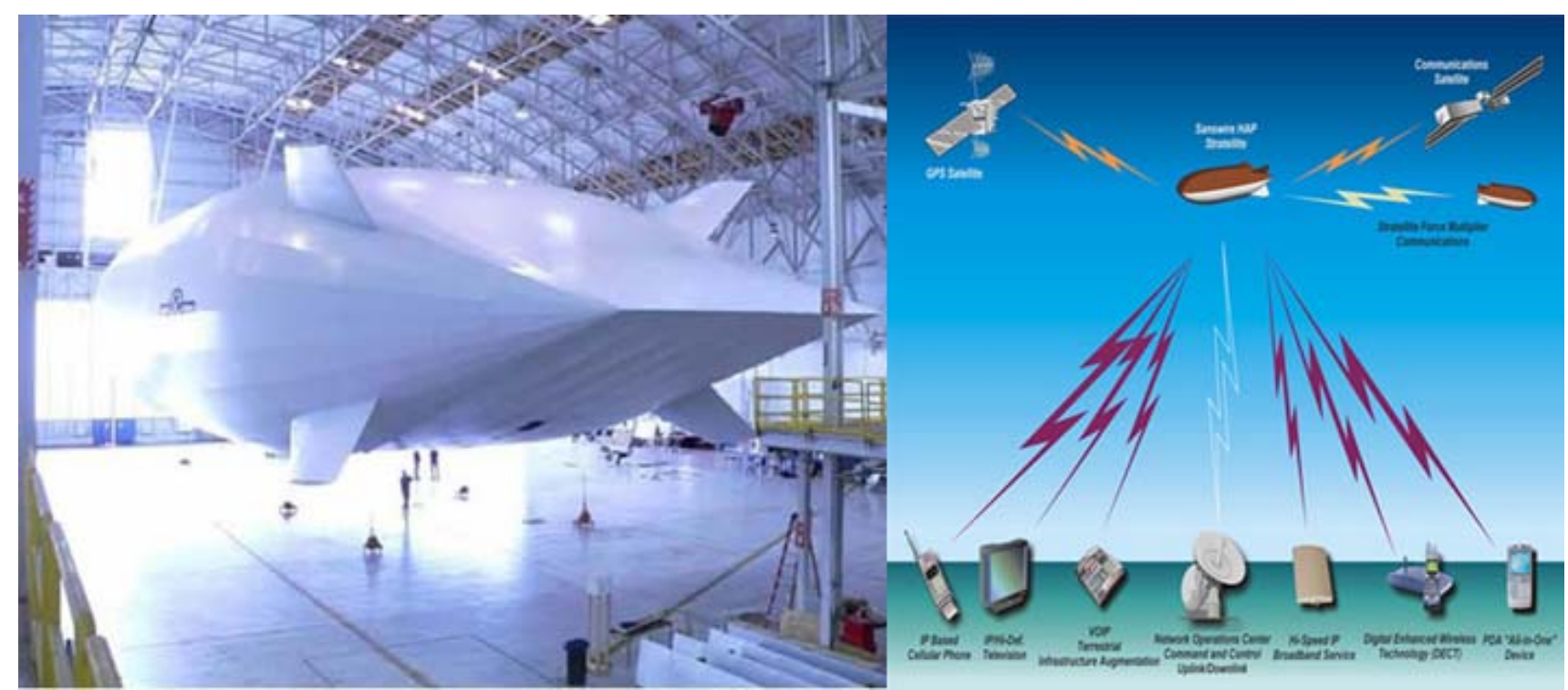

Figure 9-8: Sanswire Stratellite ${ }^{\text {TM }}$ Concept [150] 


\subsubsection{SkyPluSys UK Ltd}

SkyPluSys Ltd. was created in the UK during 2004 to develop a telecom solution using an aerostat as a platform. During a High Altitude Platforms for Communications and Other Services (HAPCOS) conference, they reported that they have signed with several Chinese customers to research and design feasible applications using High Altitude Platforms (HAPs) or tethered aerostats.[148] These applications include telecommunications services and surveillance for large events (2008 Olympics). The company also reported a partnership with Lindstand. (Lindstand, a manufacturer of lighter than air aircraft, have been involved in research and development of HAPs since 1998. [149]) Currently, there is no public information available on this company or on its current status of its research.

\subsection{Other Experiments and Research Groups}

Existing experiments and research give more of a future look into state of the art. While patents also do this, they can remain theoretical and ideological. Experiments actually prove theories and ideas to evaluate the possibility and feasibilities of those ideas.

\subsubsection{National Institute of Information and Communications Technology, Japan}

The National Institute of Information and Communications Technology (NiCT) of Japan probably has the most of experience running experiments and research with respect to airborne telecommunications. The NiCT has run the following experiments within the last 5 years:

- June-July 2002, Kauai, Hawaii

- $\quad$ Pathfinder Plus, UMTS telecommunications services at 60k ft [131]

- $\quad$ October 2002 , Hokkaido, Japan

- $\quad$ Gulf Stream II, Digital Broadcasting Test, at 36k ft [132]

- $\quad$ November 2002, YRP

- $\quad$ Broadband Access Test Using Helicopter, 1k ft [132]

- $\quad$ November 2004, Japan

- $\quad$ Japan Aerospace Exploration Agency (JAXA) Lighter than Air (LTA) Airship Telecom Mission for station keeping flight keeping [132]

- $\quad$ November 2006, Camp Roberts, California

- $\quad$ Localization Demonstration using Global Observer Prototype [132]

- $\quad$ March 2007, Saitama, Japan

- $\quad$ Broadband Relay (HD TV) with digital mechanical beam-forming antennas. [132]

There are two experiments that are most notable and relevant for providing emergency communications using an airborne platform. The first was performed on June-July 2002 in Kauai, Hawaii. This experiment tested UMTS commercial equipment on a high altitude UAV, Pathfinder Plus, at 60k ft. The second experiment occurred during November 2006 at Camp Roberts, California. This used AeroVironment's Global Observer prototype to conduct experiments to determine if aircraft can be used to locate terrestrial end users. Both experiments have important contributions into the field of knowledge for performing emergency airborne wireless communications.

The experiment in Kauai, Hawaii was extremely significant since it demonstrated that UMTS cellular communications could be conducted using a 60k ft platform with some difficulty. Figure 9-9 shows the details of the experiment. One conclusion made was the delay of the $60 \mathrm{k}$ link had caused some problems with initial access. Additionally, interference was seen at the aircraft from transmitting base stations which were over 120 miles away. Finally, wireless data at this altitude was not possible without better antennas on end user devices. These 
experiments showed that communications could be done at these altitudes; however, these problems need to be taken into consideration when deploying communications at higher altitudes. (See [131] for details on the experiment.)

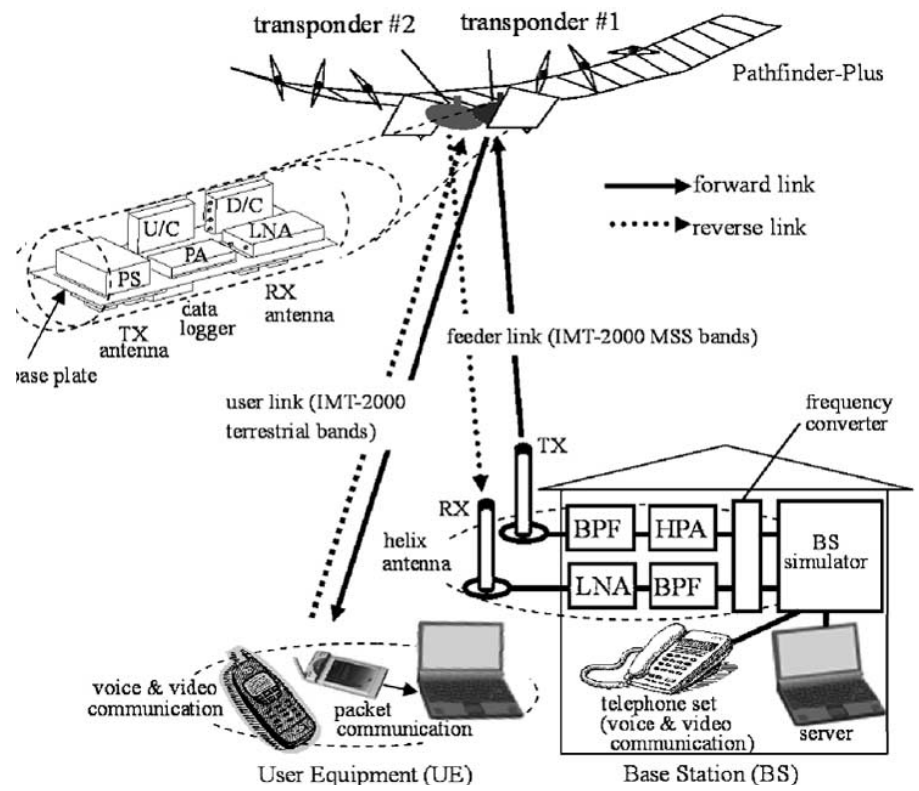

Figure 9-9: NiCT Pathfinder-Plus Experiment

The experiment at Camp Roberts used an innovative method of triangulation to locate end user transmitters on the ground. The purpose of this experiment was to evaluate if survivors of a disaster could be located by their transmitting mobile devices using an airborne platform. This was done by a combination of using known reference signals angle of arrival, on board aircraft navigation and antenna array on the wings of the aircraft. This experiment was very successful in demonstrating that the technique could work if deployed. It is likely that this solution could be a future application for UAVs in a disaster situation. The UAV used in this experiment was an AV's GO prototype. Figure 9-10 shows a pictorial representation of this experiment. 


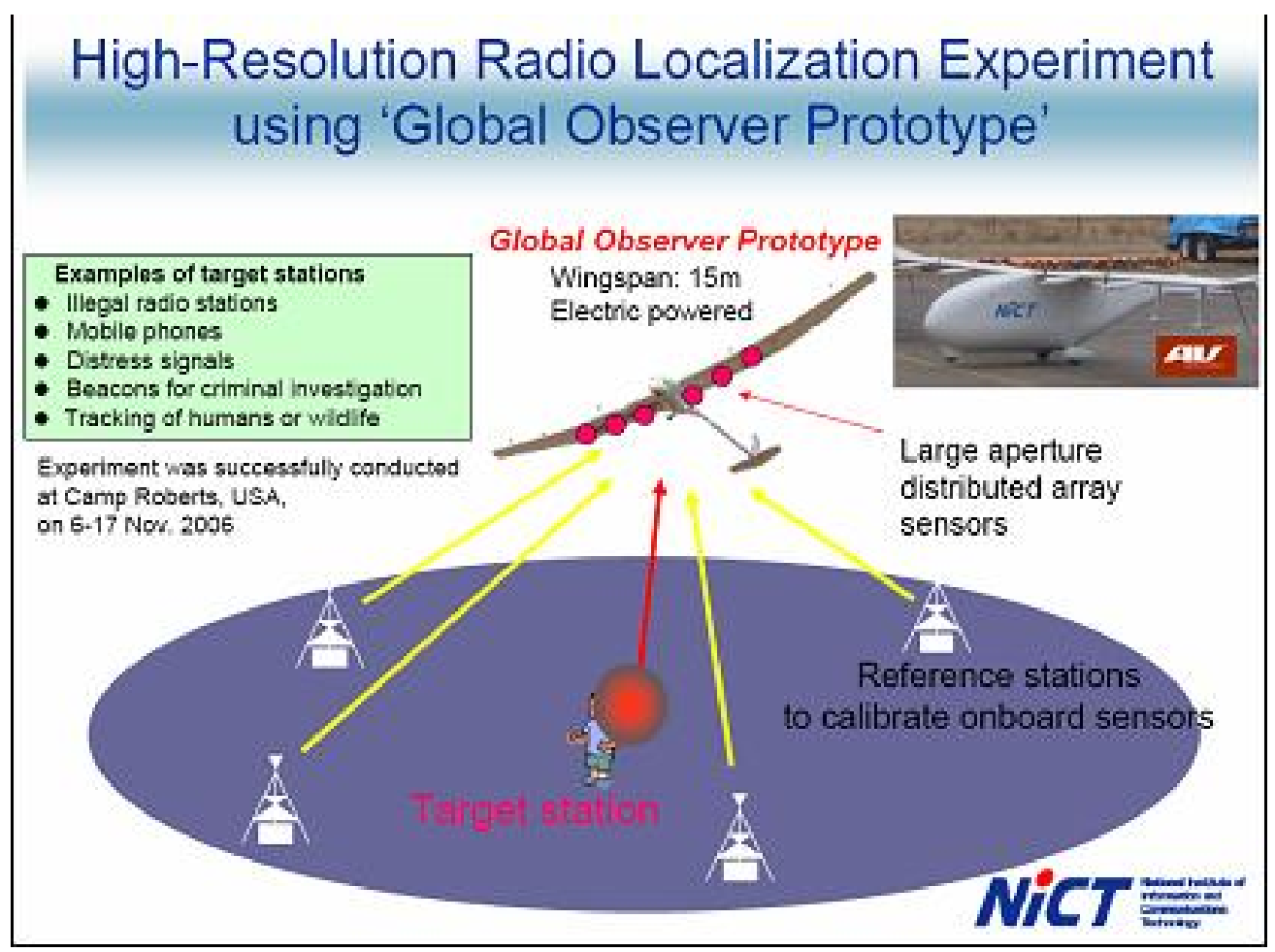

Figure 9-10: NiCT, Global Observer Prototype Experiment

\subsubsection{CAPANINA}

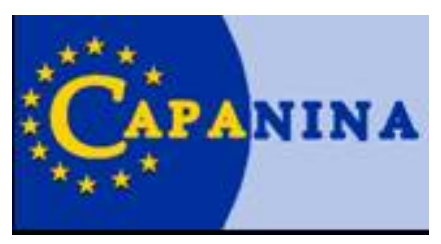

CAPANINA is a consortium of 14 different worldwide partners in academia, industry, and government whose goal is to develop wireless and optical broadband technologies for use on HAPs. This organization has also conducted successful experiments within the last few years. There are two experiments that this organization has conducted that are less relevant to providing emergency wireless communications on an airborne platform.

The first experiment, Trial 1, was conducted in Pershore, UK during August-October 2004. This experiment used a tethered aerostat at 1,000 feet and tested free space optical applications. Photos from the experiment are shown in Figure 9-11. The results from this experiment were good and improvements on the platform were made to take the aerostat to higher altitudes. 


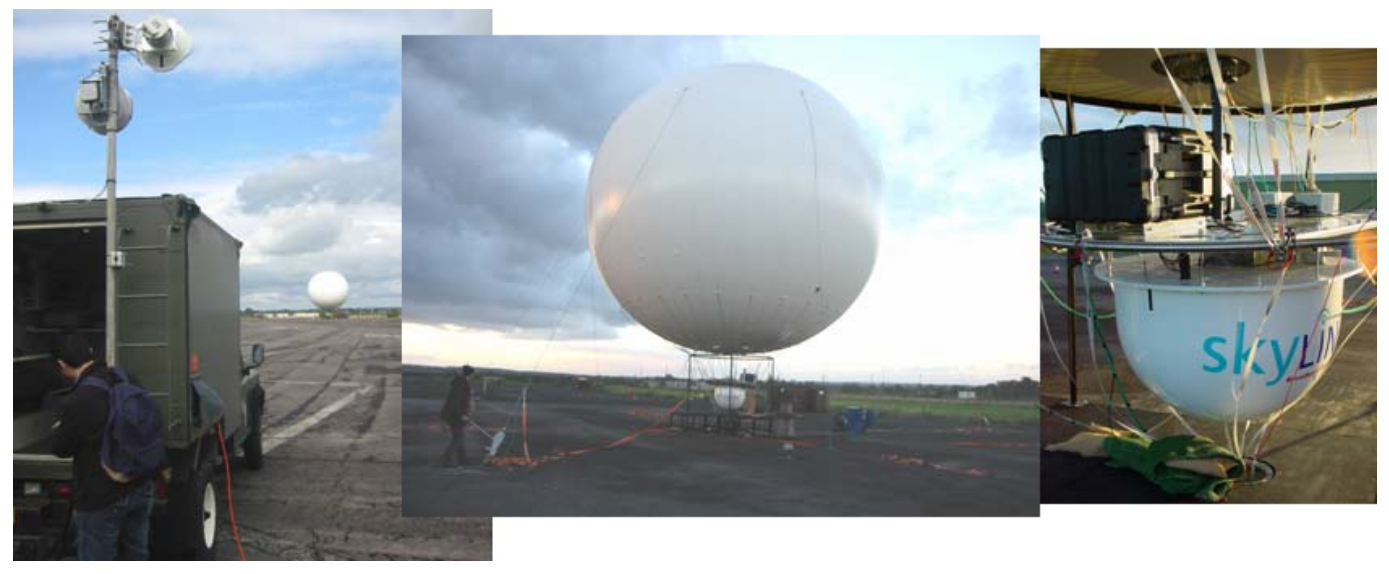

Figure 9-11: CAPANINA Trial 1

Trial 2 was conducted in Kiruna, Sweden in August 2005. The experiment consistent of a wireless LAN (802.11b) and free space optical (FSO) platform (shown middle and left Figure 9-12) using a stratospheric blimp at $\sim 60 \mathrm{k}$ feet (Shown right in Figure 9-12). This experiment was able to provide $4 \mathrm{Mbps}$ throughput using $802.11 \mathrm{~b}$ and $1.25 \mathrm{Gbps}$ link using the FSO. This experiment was important in demonstrating the capabilities of wireless and FSO potential that could be done using a stratospheric blimp.
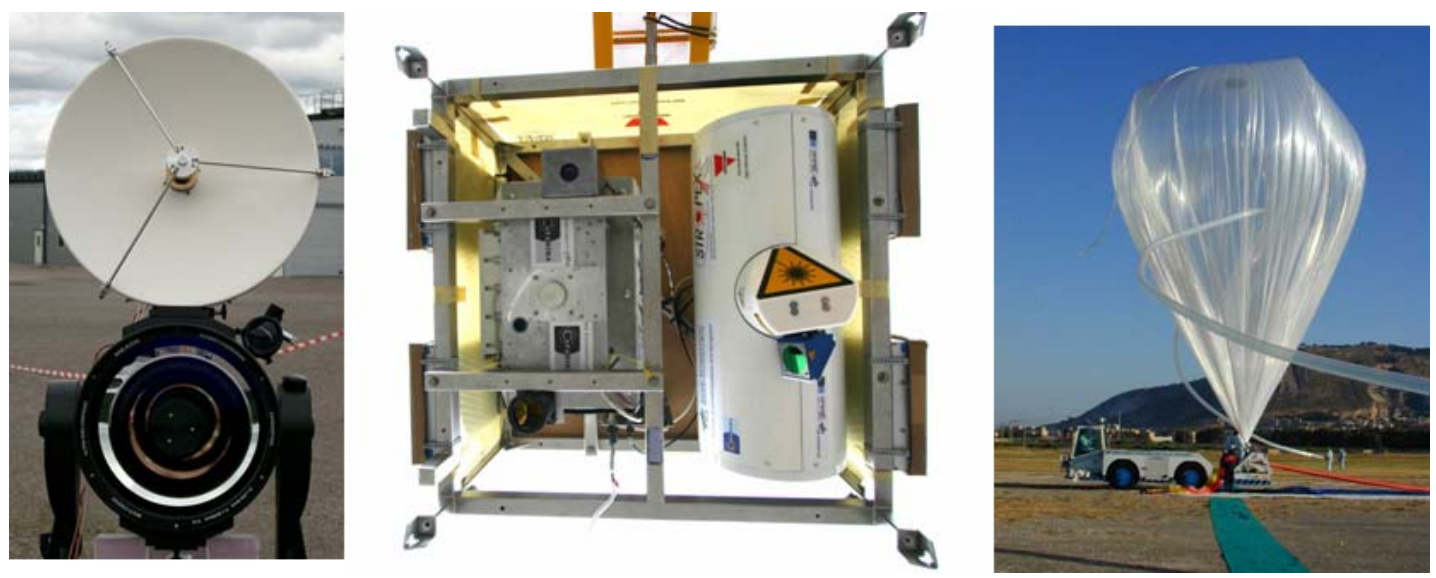

Figure 9-12: CAPANINA Trial 2

\subsubsection{COST 297-High Altitude Platforms for Communications and Other Services}

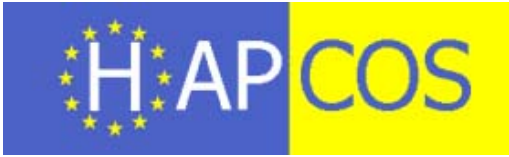

Cooperation in the field of Scientific and Technical Research (COST) is an intergovernmental network of 35 European Union (EU) member countries and one of the longest-running instruments supporting cooperation among scientists and researchers across Europe. COST research areas are divided up into groups called COST Actions each followed by a different number. [133] COST 297-HAPCOS is a one of these particular research groups which is dedicated to researching and developing new methods, analyses, techniques, and strategies for developers, service providers, system integrators, and regulators of HAPs for delivery of communications and other services. [134] This group has a large area of research dedicated to many different areas of HAP development from radio communications to platform development. 


\subsubsection{Battlefield Airborne Communications Node Program Northrop-Grumman}

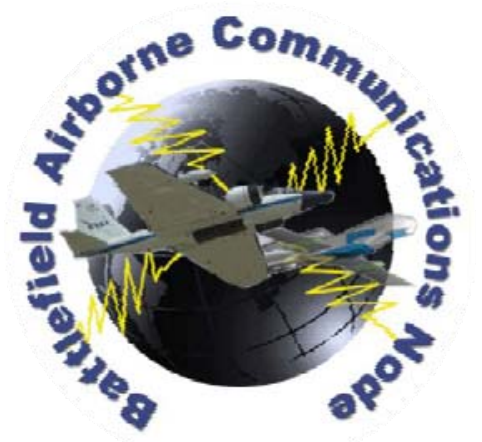

Northrop Grumman was awarded \$25.7 million, 17-month contract in April 2005 to give the government access to a broad range of technologies, capabilities, and expertise that it can rapidly apply to improve the operational readiness of fielded Defense Department systems. [136] This program became known as the Battlefield Airborne Communications Node (BACN). The program creates an IP-based airborne network using a wide variety of communications from satellite, LMR radio to cellular communications. In the spring of 2006, Northrop Grumman tested the BACN platform on the NASA WB-57 aircraft at 60k ft. [135] During this test they evaluated the possibility of using CDMA platform to make cellular calls. In this experiment they where able to make CDMA cellular calls using the Qualcomm QDBS. It is envisioned that the capabilities demonstrated on the WB-57 will make it to a High Attitude UAV platform such as Global Hawk.

\subsection{Patents of the Art}

Patents form an important part of intellectual property that must be considered when examining a future or new technology. They form a legal basis for patents from the technology that will need to be licensed, if required. It also provides a good record of the state of the art so that available technology is well known.

\subsubsection{The HALO Network ${ }^{\mathrm{TM}}$ Patent 6061562}

Raytheon patented the High Altitude Long Operation (HALO) Network ${ }^{\mathrm{TM}}$ in 2000 to provide a variety of broadband data services. The HALO network was envisioned to provide mobile broadband services to cell sites and home office buildings using a mechanically steered antenna configurations, shown in Figure 9-13. The aircraft, Proteus, flying at $51 \mathrm{k}-60 \mathrm{k} \mathrm{ft}$ and carrying $2,000 \mathrm{lb}$ of gear, would provide these services using a proprietary air interface using Local Multi-megabit Data Service (LMDS) frequencies $(28 \mathrm{GHz})$ and providing a network scaled in the $100 \mathrm{Gbits} / \mathrm{s}$. The network was predicted to support hundreds to thousands of users. Unfortunately, this vision did not come to fruition with commercial services. 

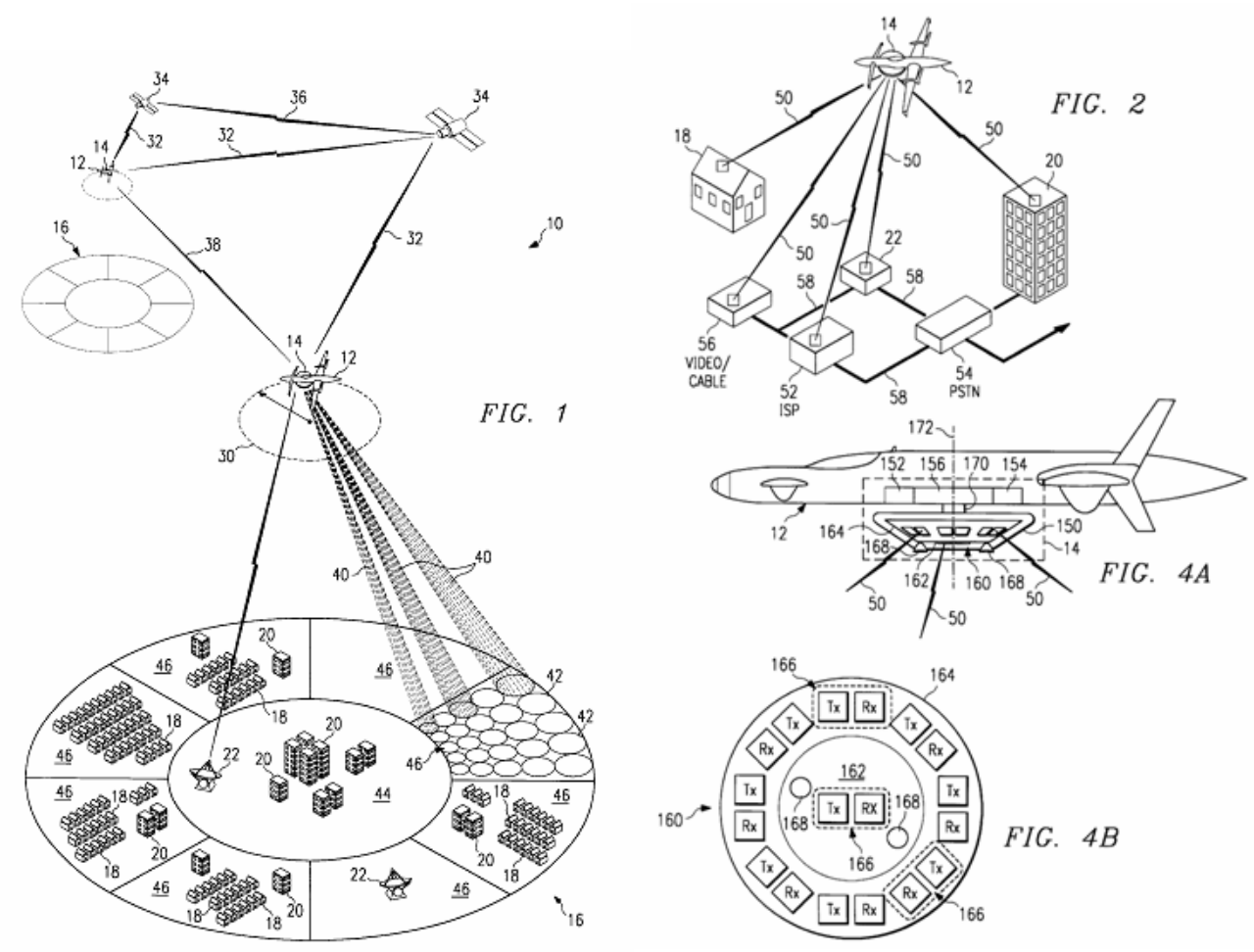

Figure 9-13: Patent 6061562

\subsubsection{Transportable Infrastructure, Patent 6804515}

Patent 6804515, filed by Motorola in 2000, patents two key strategies used for using an airborne platform for delivering cellular communications. [127] In this patent, the aircraft could be a repeating cellular communications device, see Figure 9-14, or could also be a flying base station, see Figure 9-15. This patent fundamental techniques and could be seen as an essential contribution to the art of providing cellular communications via an airborne platform. One unique aspect of this patent is that the aircraft described in the claims does not cover stratospheric aircraft. 


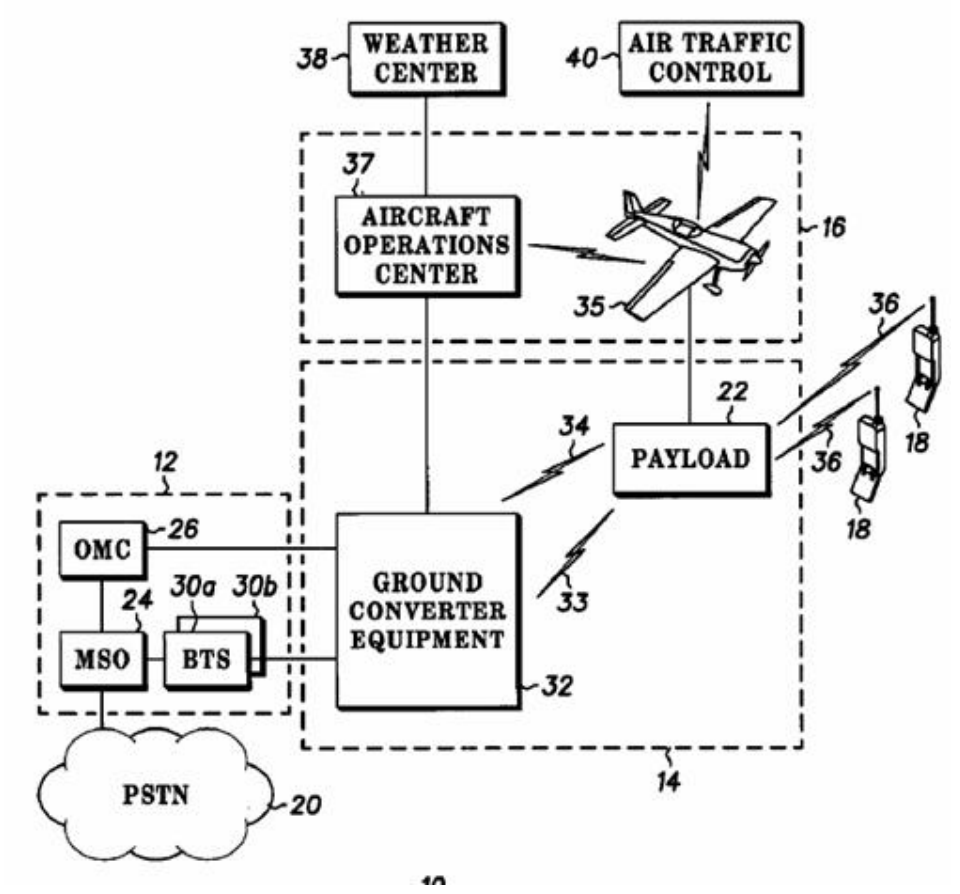

Figure 9-14: Patent 6804515, Aircraft Repeater

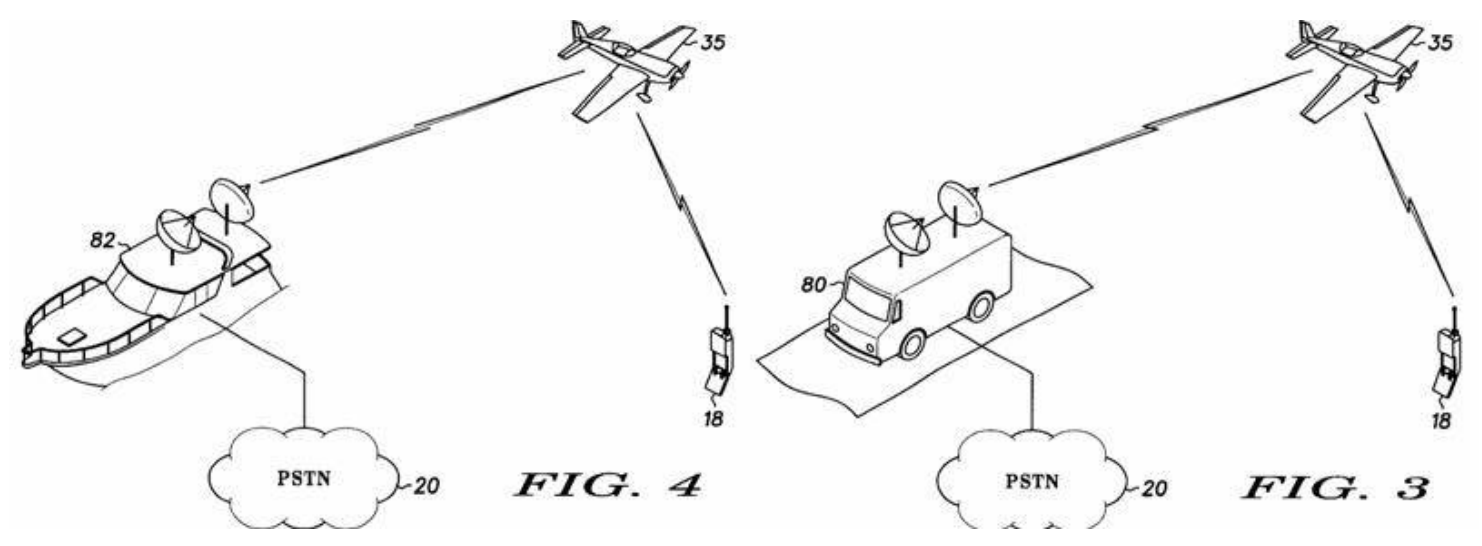

Figure 9-15: Patent 6804515, Flying Base Station

\subsubsection{Patent 6944450}

Patent 6944450, Figure 9-16, from AV covers specifically a fixed-wing aircraft flight pattern and flight station duration. This is to assume that the aircraft is most likely an UAV to maintain the operation for 3,000 hours. This patent is very closely tied to SkyTower concept that AV is pursuing with the Global Observer platform. [129] 


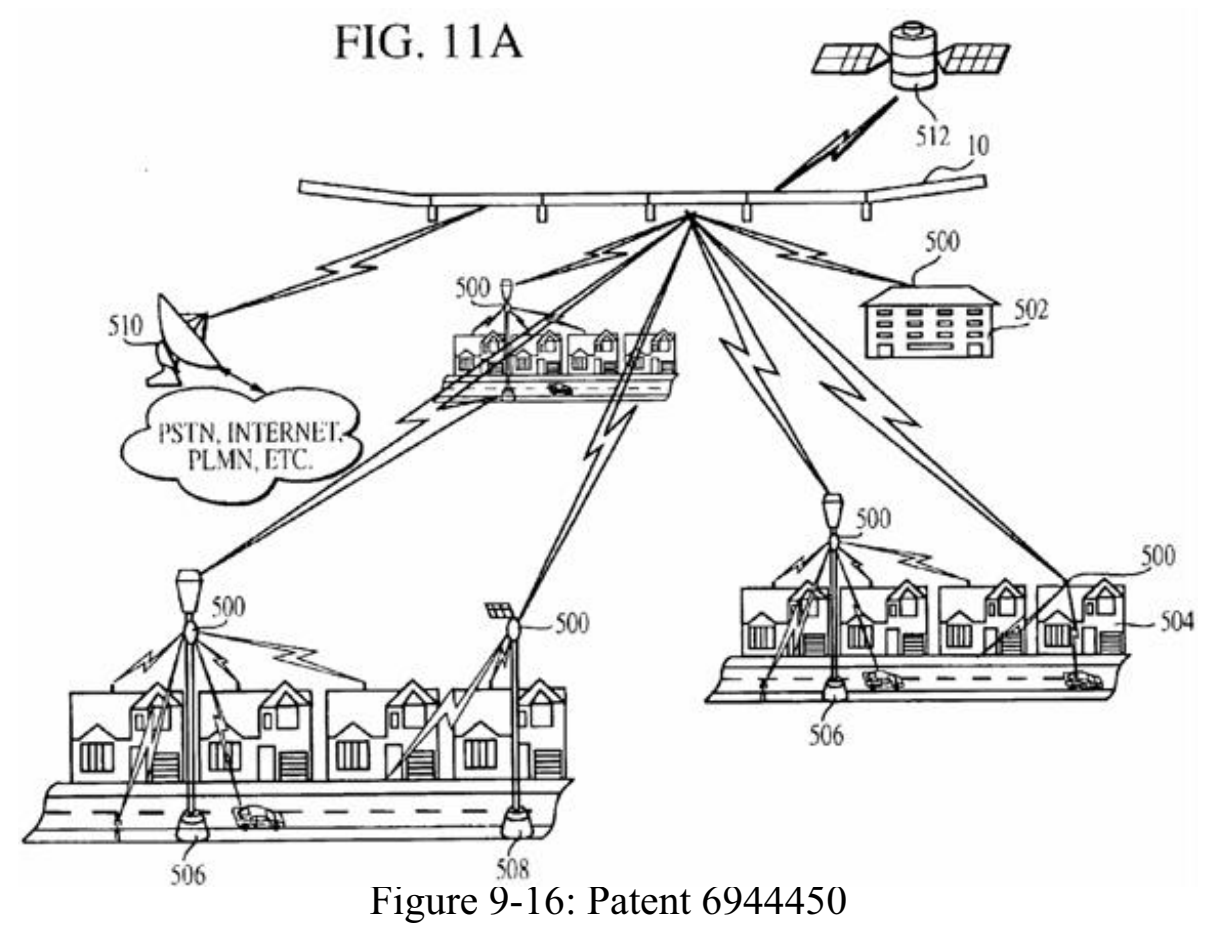

\subsubsection{Patent 6324398 Stratospheric Blimp}

The stratospheric blimp could be thought of as the "holy grail" for airborne wireless communications. The aircraft are proposed self sustaining, carry large payload, and provide large bandwidths and coverage areas. Lucent has patented the idea for the stratospheric blimp in Patent 6324398, which covers how power systems and telecommunications would be provided using such a system.[130]

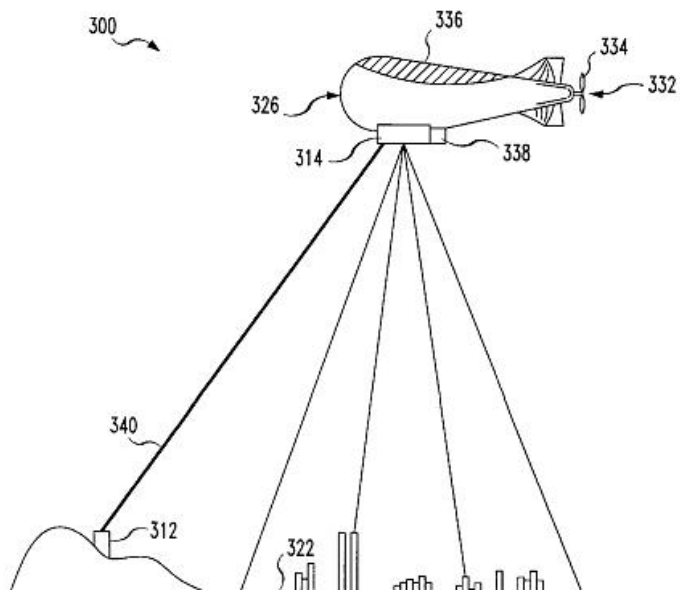

Figure 9-17: Patent 6324398, Stratospheric Blimp

\subsection{Summary and Conclusions}

After reviewing and investigating the prior art the following conclusions were made:

- $\quad$ Turnkey ACNs are already available: Aerofone, Platform Wireless and TECORE/TCOM are offering these solutions.

- $\quad$ Turnkey ACN will still need a spectrum agreement with the operator.

- $\quad$ Existing emergency communications systems have very similar architectures and components. 
- Existing experiments and research have been focused on stratospheric aircraft for communications, experimentation is occurring worldwide in this area. 


\section{REFERENCES}

[125] Martin, James N. et al., 2000, "Wireless Ccommunication Uusing an Aairborne Sswitching Nnode," James N. Martin et al, Patent number: 6061562, Filing date: Oct 30, 1997, Issue date: May 9, 2000, http://www.google.com/patents?id=plkEAAAAEBAJ\&dq=6061562

[126] The HALO Network ${ }^{\mathrm{TM}}$, Nicholas J. Colella, Angel Technologies Corporation, James N. Martin, Raytheon Systems Company, Ian F. Akyildiz, Georgia Institute of Technology, IEEE Communications Magazine, June 2000, pp. 142-148.

[127] McCraw, Dawn M. et al., 2004, “Transportable Infrastructure for Airborne Cellular System,” Patent 6804515, October 12, 2004, http://www.google.com/patents?id=SesRAAAAEBAJ\&dq=6804515

[128] Cox, Earl C., Communications System," Patent 6944450, September 13, 2005, http://www.google.com/patents?id=pDkWAAAAEBAJ\&dq=6944450

[129] AeroVironment, "Planned SkyTower Telecom System Concept," http://www.aerovironment.com/AV Docs-qry.asp?avDIV=224

[130] Louis J. Lanzerotti et al, 2001, "Wireless Telecommunications System having Airborne Base station," Patent 6324398, November 27, 2001, http://www.google.com/patents?vid=USPAT6324398

[131] Oodo, Masayuki et al., "Experiments on IMT-2000 Using Unmanned Solar Powered Aircraft at an Altitude of 20 km,” IEEE Transactions On Vehicular Technology, Vol. 54, No. 4, July 2005.

[132] Hiroyuki TSUJI , National Institute of Information and Communications Technology (NICT) Japan, "Recent Developments for HAPS Applications in Japan," HAPCOS Conference Proceedings, November 13,2006.

[133] European Cooperation in the field of Scientific and Technical Research (COST), http://www.cost.esf.org/

[134] High Altitude Platforms for Communications and Other Services (HAPCOS), "COST 297," http://www.hapcos.org/

[135] Northrop-Grumman, "BACN CDMA Subsystem Test Results,” Northrop-Grumman, June 02, 2006

[136] Space Daily AFP, "Northrop Grumman aAnd USAF Conduct Tests Oof Airborne Networking System," Space Daily AFP, San Diego CA (SPX), Dec 14, 2005, http://www.spacewar.com/reports/Northrop_Grumman_And_USAF_Conduct_Tests_Of Airborne Networking_System.html

[137] AeroFon, http://www.aerofon.com/default.htm

[138] AeroFon Corp. \& QUALCOMM Government Systems Group, “Airborne CDMA Cellular System Demonstration Report," May 29, 2003, http://www.aerofon.com/AeroFon\%20Demonstration\%20Report\%20final.pdf

[139] AeroFon Corp, "Airborne Repeater Proof-of-Concept Final Report," https://www.rcs800mhz.org/web/generalreports_aerialfinalreport.pdf

[140] TECORE Press Release, "TECORE and TCOM Unveil Rapidly Deployable Airborne Emergency Cellular Communications System at MILCOM 2006," http://tecore.com/page/602/show_item/94/News.htm

[141] TECORE Wireless Systems, "Rapid Response Systems,” http://tecore.com/index.php?id=539

[142] TCOM, “TCOM's 17MTM Aerostat System,” http://www.tcomlp.com/17M.html 
[143] Airborne Relay Communications "ARC" System, Platforms Wireless International http://www.plfm.net/pdf/plfm/arc_web_site.pdf

[144] Platforms Wireless International, "The Aerostat Airborne System Delivery Platforms," http://www.plfm.net/pdf/plfm/aerostat_web_site.pdf

[145] Platforms Wireless International Corporation, "Platforms Wireless Announces First Sales of ROSETTA911(TM) Interoperable Communications Systems," http://www.plfm.net/newsletter.php?id=07 07-30Rosetta 911 first sales.php

[146] AeroVironment, "UAS Advanced Development Center: Global Observer (2004 to present)," http://www.aerovironment.com/ADC_Project_Details.asp?Prodid=35

[147] Aerovironment, "SkyTower Concept," http://www.avinc.com/downloads/GO01 AV GO SkyTower Web_pdf

[148] Chun Lu, SkyPluSys, “HAPs Development in China,” COST297-0107-WG30-PRI-P01

[149] Lindstand, "High Altitude Long Endurance (HALE) Aerostatic Platforms," http://www.lindstrand.co.uk/hale.html

[150] Sanswire, “About the Stratellite," http://www.sanswire.com/stratellite.htm 


\section{ACRONYMS}

\begin{tabular}{ll} 
ACS & Aero Communications System \\
AV & AeroVironment \\
BACN & Battlefield Airborne Communications Node \\
BTS & Base Transceiver Station \\
CDMA & Code Division Multiple Access \\
COST & Cooperation in the field of Scientific and Technical Research \\
EU & European Union \\
FEMA & Federal Emergency Management Agency \\
FSO & free space optical \\
GO & Global Observer \\
GSM & Global System for Mobile \\
HALO & High Altitude Long Operation \\
HAP & High Altitude Platform \\
HAPCOS & High Altitude Platforms for Communications and Other Services \\
HLR & Home Location Register \\
IP & Internet Protocol \\
JAXA & Japan Aerospace Exploration Agency \\
JISCC & Joint Incident Site Communications Capability \\
LAN & local area network \\
LDMS & Local Mutli-megabit Data Service \\
LTA & Lighter than Air \\
NiCT & National Institute of Information and Communication Technology \\
NORTHCOM & U.S. Northern Command \\
PSAP & Public Safety Answering Point \\
PSTN & Public-Switched Telephone Network \\
QDBS & Qualcomm Deployable Base Station \\
RDN & Rivada Deployable Networks \\
UAV & Unmaned Aerial Vehicle \\
\hline
\end{tabular}




\section{Policy Issues}

To deploy an airborne emergency wireless communications capability, challenges with policy issues outweigh the technical ones. Although the main goal of the report is to analyze the technical feasibility of emergency wireless airborne communications, the policy issues represent a tremendous hurdle to overcome. These issues are captured in this section and proposals for solutions to the issues are also presented.

\subsection{Using Cellular Frequency Spectrum}

Most COTS equipment uses commercial spectrum frequencies. The U.S. Government cannot freely operate on these frequencies, even in times of a major disaster. Commercial spectrum is a capital asset owned by the cellular network operators which is licensed and auctioned by the Federal Communications Commission (FCC). In addition to the spectrum being a capital asset to the operators, it is also viewed as a public asset.

To use this asset, there must be some coordination between the cellular operators and the U.S. Government agency providing the emergency communications system. This is primarily due to interference. Interference is where neighboring cellular carriers cause interference with each other due to operation in same or neighboring frequency bands. However, there are things that can be done to insure there is little or no interference to commercial cellular networks.

\subsubsection{Use an Agent}

Mobile Virtual Network Operators (MVNO), such as Virgin Mobile ${ }^{\circledR}$, do not actually own infrastructure or spectrum. They lease network time from operators who own infrastructure and spectrum then resell minutes to their customers. Using this business model, it is possible to use commercial spectrum owned by the actual license holders. Operating as an MVNO also requires that phones are registered with a specific Home Location Register (HLR) for roaming purposes. For example, a person who uses an MVNO may use other commercial networks when in service and could use a private network if one was setup by the MVNO during a catastrophic event. USNORTHCOM, FEMA, and the Air National Guard all use an agent when negotiating for spectrum usage.

\subsubsection{Executive Order}

The easiest way to use cellular frequencies is with an executive order from the president of the United States. Under Title 47: Telecommunication Section 201.3:

"President is authorized ... during war ... [to] suspend or amend rules and regulations, close stations and facilities, and authorize U.S. government use and control of telecommunications resources with regard to ... Radio communications" [151]

However, in the event of a catastrophic event, such as Hurricane Katrina, this would not be the case since it is a natural disaster rather than an act of war. In the event of a major terrorist attack, such as an improvised nuclear device, it is debatable whether or if the president could invoke this power.

\subsubsection{Space Division Multiplexing and Gap Filling}

Space Division Multiplexing (SDM) is the ability for antennas or wireless systems to use different frequencies in distinctly different areas. This could be done by adjusting a radiation pattern of a beam-forming type of antenna. Using beam-forming technologies, it is possible to "gap-fill" areas that are experiencing outages due to a catastrophic event. However, this requires important information to be known ahead of time. Critical database parameters would need to be available, which can be loaded onto the aircraft when a mission is required. Status of the ground assets (functional and Out of Service [OOS]), must be determined through communication with the Network Operations Center (NOC). When these outage areas are determined, the aircraft forms the coverage beams appropriately and provides coverage while in flight. Once the outage is over, the system could detect this and stop providing coverage. Figure 10-1 shows a wide-area Base Transceiver Station (BTS) outage. The ACN flies into the affected area and places "beams" in each of the outage areas. It has knowledge of the outages from the NOC outage report. This report could either be a wireless data interface or air-to-ground voice communications. 


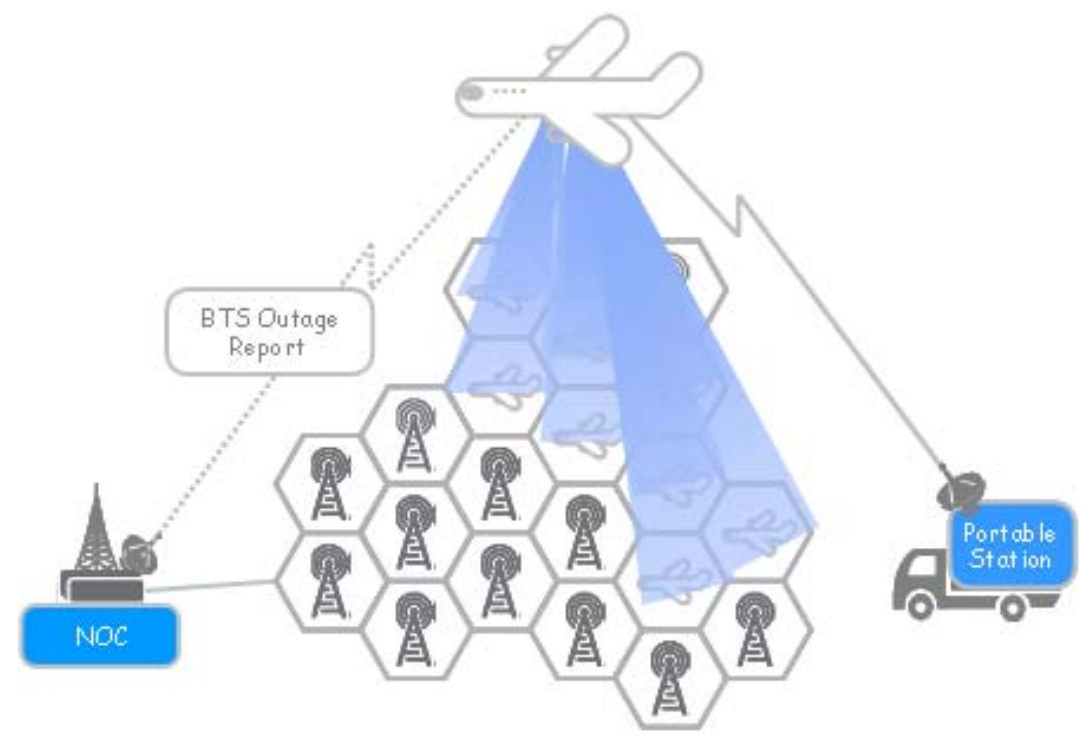

Figure 10-1: Space Division Multiplexing using beam forming antennas.

\subsubsection{Conclusion}

In general, cellular operators want to assist during a disaster. During Hurricane Katrina, many competing cellular operators worked together by sharing resources in order to restore communications to the best of their ability. Additionally, cellular operators also cooperated with United States Northern Command (USNORTHCOM) and Federal Emergency Management Agency (FEMA) to allow for stand-alone Cell on Wheels (COW) systems to be operated near their service area. However, this is the policy issue that must be solved before the U.S. government can provide a cellular communications during a catastrophic event.

\subsection{Logistical Issues}

\subsubsection{Cellular Phones Access Methodology}

Once an ACN communications platform is deployed, it will ideally provide two essential roles for cellular communications functions: the first responder's critical communications and 911 calls. First responder communications require private access into the network. This could be done with Wireless Priority Service (WPS) or by allowing exclusive access via an HLR configured to only allow specific users access to the network. This necessitates any user who requires access into the system to have their everyday phone information entered into the HLR. If the local communities have not already purchased cellular services with this capability, the responding federal agency will need to issue appropriate phones to all the first responders or configure the system to allow access for the devices. For E911, any functional cellular phone should work as long as it is compatible with the architecture used in the airborne system.

\subsubsection{Phone Numbering Plan}

Regardless of the phone access method, a directory of all users on the network will need to be identified and created. This could be a paper printout of all users and their phone numbers or a look up capability and application built into each phone that has access to the network. An example of this application would allow each user to complete a form on their phone or computer, which requires them to enter their organization, name, and pertinent contact information. This information would then make its way into a centralized database or server for later recall. Regardless of the implementation, first responders will need a method to contact each other.

\subsection{Coordination with Federal and Commercial Assets}

Creating a master coverage plan using federal and commercial assets will be important. FEMA, USNORTHCOM, and Air National Guard all have deployable cellular equipment. Carriers also have similar 
capabilities, all of which are deployable in the event of a disaster. Coverage must be coordinated holistically with other agencies such that coverage is equitable and non-interfering. This should be closely coordinated with cellular operators since they will have current information on outage areas and places where assets would be required. This coordination should be done to refine coverage plans and mitigate technical issues associated with deploying cellular networks adjacently.

\subsection{Stake Holder Agencies}

Multiple organizations at the federal, state, local, and tribal levels are involved in development of interoperable and emergency communications. To enhance the deployment of an airborne emergency communications system, the Department of Defense (DOD) should endeavor to educate these multiple agencies of the system's operational capabilities and limitations. Based on the INL research and briefings, the following is a list of the organizations that need awareness:

- National Communications System

- Federal Communications Commission

- National Emergency Number Association (NENA)

- Association of Public Safety Communications Officials International (APCO)

- National Association of State Telecommunications Directors (NASTD)

- Council of State Governments

- National Governor's Association

- National Public Safety Telecommunications Council (NPSTC)

- Public Safety Wireless Advisory Committee (PSWAC)

- FCC Public Safety National Coordination Committee

- National Security Telecommunications Advisory Committee (NSTAC)

- The Next Generation 911 Organization

- Wireless Communications Alliance (WCA)

- Commercial Telecommunications and Internet Association (CTIA).

\subsection{Airspace Coordination and ESF \#1}

Airspace coordination during a catastrophic event is part of a much larger issue that will become important. After Hurricane Katrina, there was no air traffic control available for 3 days. Pilots relied on line-of-site radio communications and to de-conflict flight operations. Plans for airspace coordination will need to be addressed in advance with the Department of Transportation (DOT), which is the champion of Emergency Support Function (ESF)-1-Transportation. [151] It is likely that there may be many issues with flying aircraft during after the disaster occurs. Without any pre-coordination with the Federal Aviation Administration (FAA) and DOT concerning ESF-1, there could be many problems with deploying an ACN.

\subsection{Integration into ESF \#2}

Emergency Support Function 2 (ESF-2) is best described in the National Response Plan (NRP) communications annex:

"Emergency Support Function (ESF) 2 - Communications ensures the provision of Federal communications support to Federal, State, local, tribal, and private-sector response efforts during an Incident of National Significance. This ESF supplements the provisions of the National Plan for Telecommunications Support in NonWartime Emergencies, hereafter referred to as the National Telecommunications Support Plan (NTSP).’[151] 
Because the ACN is involved in ESF-2, an operation plan must be developed. Functional organizations who participate in ESF-2 must understand that the capability exists and the operational procedures to use such an asset.

\subsection{Conclusions and Recommendations}

This section does not include all issues associated with deploying an ACN, only the issues uncovered during the scope of the technical research. While INL has presented some strategies for overcoming some of the policy issue, it is up to the U.S. government agencies who will need to resolve the issues mentioned.

The issues are summarized as:

- Determine how commercial cellular spectrum can be used so that it can be legally used by DOD. USNORTHCOM, FEMA, and National Guard Bureau currently use and agent. It is recommended that agencies leverage and expand existing agreements to insure interoperability.

- Integrate and test a complete emergency framework holistically.

- $\quad$ Establish policy and strategy for emergency cellular coverage. Following an event, coordinate coverage for the equitable benefit of the citizens must and to minimize interference. It is recommended to complete an agreement between federal agencies and cellular operators. The NSTAC, Emergency and Interoperable Communications Task Force is a potential place to begin this discussion.

- Make the first responder agencies aware of the backup communications capabilities that can be provided in the event of a disaster or catastrophic event.

- $\quad$ Coordinate the airspace issues for an ACN during a catastrophic event with DOT and FAA. It is recommended that a Memorandum of Understanding be signed between the DOD, DOT, and FAA.

- $\quad$ Brief the NCS and NSTAC on the capability or additional capabilities that the federal government has for disaster response.

- $\quad$ Review the NRP with regard to the ACN deployment issues. 


\section{REFERENCES}

[151] Code of Federal Regulations, Title 47, Section 201.3.e , Policy, http://www.gpoaccess.gov/,

[152] U.S. Department of Homeland Security, "National Response Plan," December 2004, http://www.dhs.gov/xprepresp/committees/editorial 0566.shtm, 


\section{ACRONYMS}

ACN

APCO

BTS

COTS

COW

CTIA

DOD

DOT

ESF

FAA

FCC

FEMA

HLR

INL

MOU

MVNO

NASTD

NENA

NOC

NPSTC

NRP

NSTAC

NTSP

OOS

PSWAC

SDM

USNORTHCOM

WCA

WPS
Airborne Cellular Node

Safety Communications Officials International

Base Transceiver Station

Commercial off the Shelf

Cell on Wheels

Telecommunications and Internet Association

Department of Defense

Department of Transportation

Emergency Support Function

Federal Aviation Administration

Federal Communications Commission

Federal Emergency Commission

Home Location Register

Idaho National Laboratory

Memorandum of Understanding

Mobile Virtual Network Operators

Association of State Telecommunications Directors

National Emergency Number Association

Network Operations Center

National Public Safety Telecommunications Council

National Response Plan

National Security Telecommunications Advisory Committee

National Telecommunications Support Plan

Out of Service

Public Safety Wireless Advisory Committee

Space Division Multiplexing

United States Northern Command

Wireless Communications Alliance

Wireless Priority Service 


\section{Modeling and Simulation}

In order to assess the operational capabilities and limitations associated with an emergency airborne wireless communications system this project required the ability to model, simulate and evaluate wireless communications and electrical infrastructures, their interdependencies, and the consequences and cascading effects on them due to natural or man-made hazards. We designed a simulation framework for providing a distributed "plug-and-play" environment that allowed for integration of existing best-in-class hazard and infrastructure sector models to produce the Critical Infrastructure Protection and Resilience Simulator (CIPRsim). The CIPRsim is a scalable integration of existing and proven models for potential hazards such as hurricanes, earthquakes, and wild fires; physics-based models for infrastructures such as cellular communications and electrical transmission and distribution networks; damage assessments due to effects of hazards on infrastructure components; and methods to determine and simulate infrastructure interdependencies in a robust, high-fidelity, intuitive graphical user interface.

\subsection{Electrical and Telecommunications Interdependencies}

Interdependency is defined as "the bi- or multi-directional reliance of an asset, system, network, or collection thereof, within or across sectors, on input, interaction, or other requirement from other sources to function properly." [153] The criticality of understanding and evaluating the interdependencies related

to critical infrastructure sectors is well recognized. There are numerous documents stating the importance of interdependencies as well as studies and tools developed to address the need for greater understanding of the cascading effects due to failure. [154] [155] Many of these documents underscore the clear recognition at senior policy levels of the importance of understanding interdependencies in critical infrastructures/key resources $(\mathrm{CI} / \mathrm{KR})$ so that our nation is fully prepared to respond to natural or manmade disasters. Of particular relevance to this specific effort are the reports discussing the interdependencies between the electric power grid and telecommunications by the President's National Security Telecommunications Advisory Committee (NSTAC) [156], [153] that discuss national security and emergency preparedness issues associated with the interdependencies between these two sectors. These reports address the Administration's concerns that telecommunications and electric power interdependencies may create vulnerabilities, particularly in emergency response situations. [153]

The complexity of the electrical and telecommunications infrastructures and their interdependencies make modeling and simulation $(M \& S)$ important elements for analysis related to the concerns raised by the NSTAC. [155] There is an urgent need for a high-fidelity, dynamic modeling and simulation framework that illuminates greater understanding of the effects of disasters upon infrastructure components.

\subsection{CIPRsim}

CIPRsim provides a method for linking disparate models and analysis capabilities through a distributed environment utilizing the IEEE 1516 High Level Architecture (HLA) standard for simulation communication and time/event management. There are several advantages in applying this approach to infrastructure analysis. It allows the flexibility to use best-in-class models developed by domain experts regardless of where they reside. By incorporating these best-in-class models and analyses into a single simulation with a common temporal and spatial context, decision makers are provided with an integrated checks-and-balance system where every module dynamically interacts with each other at all time steps throughout the simulation. This approach of dynamic integration provides the basis in which to model interdependencies between various critical infrastructure sectors and their failures, determine possible cascading effects due to these interdependencies, and analyze how these interdependencies may affect both military and civilian response operations in a "play-by-play" fashion. This differs from other infrastructure interdependency simulations that use a serial process whereby the results of one 
infrastructure simulation are fed into the next simulation, the outcome being a one-way dependency [153] analysis that fails to show the two or three-dimensional dependencies such as those that exist between the electrical and communications infrastructures [155]. The distributed and dynamic interdependency simulation approach incorporated in CIPRsim results in a situational awareness portrait of how a disaster scenario will play out in real-time and what infrastructure components will be affected at various times throughout the course of man-made or natural events.

\subsubsection{Technical and Functional Requirements}

The CIPRsim critical infrastructure modeling and simulation framework was designed and built with robustness and extensibility in mind. This includes both flexibility in the type of platform that the software runs on as well as scalability in regard to providing "plug-and-play" capabilities for incorporating multiple hazard and Critical Infrastructure (CI)/Key Resource (KR) sector models into larger comprehensive simulations. Due to its platform independence, Java is the preferred programming language for the application, which allows for execution on Windows 2000/XP, Linux, and Solaris platforms. To address the requirement of scalability, the CIPRsim framework is based upon the IEEE 1516 HLA standard for federated simulation. The Department of Defense (DoD) led the development of the HLA in the 1980s and 1990s to address its need for integrated, interoperable and reusable distributed simulation models in war-gaming scenarios. [157] DoD's efforts regarding HLA were driven primarily for the purpose of being able to use and reuse existing "best of breed" simulations in new combinations to avoid developing any single, monolithic architecture with the impossible goal of meeting all simulation needs.

While the history of the HLA is deeply embedded within the DoD and war-gaming activities, it is widely recognized as representing the state of the art technology for the integration of distributed simulation models [158] for a diverse range of focus areas such as emergency response [159], [160] and urban chemical disasters. [161] In addition to these applications, Idaho National Laboratory (INL) recognized HLA as a viable means to base dynamic and distributed simulations and analyses related to critical infrastructure systems as it provides a common architecture for component-based simulations where multiple simulations are combined to provide a more comprehensive simulation. Components, or in the case of CIPRsim, models, within the HLA framework are known as federates, which collectively, operate within a federation that can run across multiple computers. Figure 11-1: CIPRsim HLA Schematic illustrates the general HLA schematic for CIPRsim indicating the various federates. 


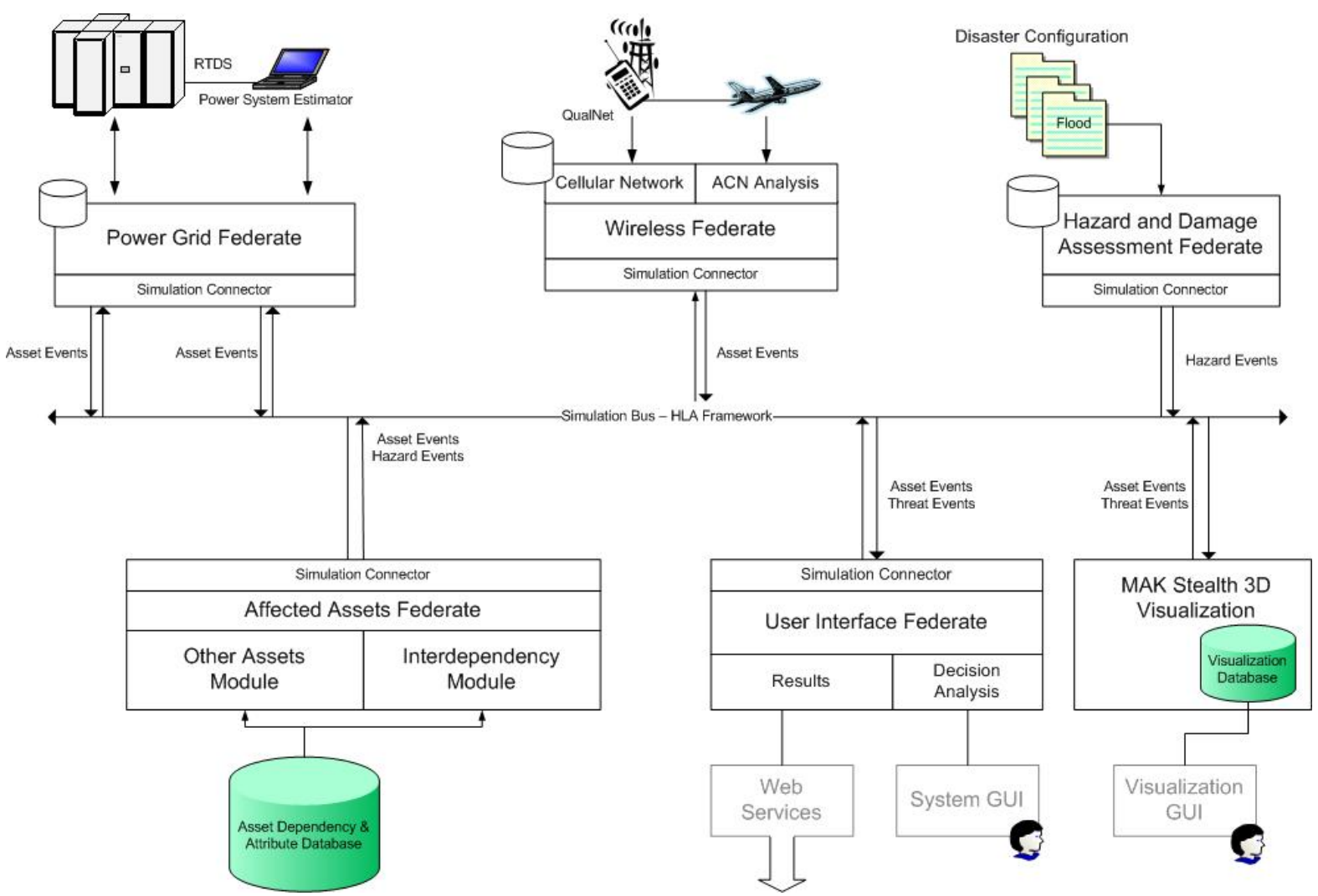

Figure 11-1: CIPRsim HLA Schematic

This federated design is based on addressing several of the following assumptions that were considered during requirements definition for CIPRsim [157]. These assumptions include:

- $\quad$ No single simulation can satisfy the needs of all users due to differences in interests and requirements for fidelity and detail

- Simulation developers vary in their knowledge across domains to be simulated

- $\quad$ No one can anticipate all the uses of a simulation and all the ways simulations could be usefully combined

- $\quad$ Simulations must allow for future technology and tools to be incorporated.

By addressing these assumptions, the HLA provides a very flexible and scalable basis for comprehensive simulations within the critical infrastructure protection and analysis arena. Implementation of the HLA allowed CIPRsim to meet many of the requirements of the application that were specified by the design team. These include:

- Capability to incorporate existing relevant and community accepted and validated physical models. It is intended that the framework, with specified object model agreement, allow for "models of choice" to be used in the simulation to allow best-in-class models to be utilized as they become available. This scalability includes integration of multiple hazard and infrastructure models.

- Capability for distributed simulation. It is intended that the framework allow for capitalization of geographically distributed domain expertise and computing power.

- Capability to provide an "All-Hazards" simulation environment. The hazards that can potentially affect infrastructure must not be limited to specific classes of events. Currently the framework 
integrates hazards due to hurricanes such as storm surge, flooding, high winds [162], [163], and ground motion from earthquakes.[164][153] However, it is designed to support a full range of plausible hazards. It also allows users to manually influence assets and determine consequences for individual component-level failure analyses.

- $\quad$ Supports real-time interaction between the models. This includes both temporal and spatial synchronization of the models to achieve greater understanding of infrastructure status during an event, the capability for time-stepped interdependency analysis, meaning that the framework gives the user not only the final result, but also provides a "play-by-play" throughout the simulation allowing for information as to the specific chronology of events.

It was essential that the CIPRsim maintain interoperability with existing user community situational awareness tools and common operating pictures (COPs). These requirements include:

- Interoperability and information exchange with existing end-user community analysis tools and COPs, including the U.S. Department of Homeland Security's Integrated Critical Asset Viewer (iCAV), U.S. STRATCOM's Strategic Mission Assurance Data System (SMADS) and the National Geospatial Intelligence Agency's (NGA) Palanterra.

- $\quad$ Capable of ingesting, managing, and performing additional analysis using industry standard geospatial data formats. This includes capability to manually load, visualize and manipulate userspecific data sets, such as:

- $\quad$ Raster data sets (GeoTIFF, ENVI, Imagine, etc.)

- $\quad$ Point-based data (shapefiles, geodatabase)

- Vector or line data (shapefiles, geodatabase)

- $\quad$ Polygon data (shapefiles, geodatabase)

- $\quad$ Conform to Open Geospatial Consortium (OGC) specifications as noted in the Defense Critical Infrastructure Program's (DCIP) Geospatial Data Strategy [165] and the National GeospatialIntelligence Agency's Geospatial Intelligence Standards [166] related to implementation of Web services for sharing information in a net-centric fashion. These OGC specifications include:

- $\quad$ Web Map Server (WMS). Produces maps of spatially referenced data dynamically from geographic information. These maps are not the data itself, but are generally rendered in a pictorial format such as PNG, GIF, or JPEG. [167]

- Web Coverage Service (WCS). Provides access to potentially detailed and rich sets of geospatial information, in forms that are useful for client-side rendering, multi-valued coverages, and input into scientific models and other clients. [168]

- Web Feature Service (WFS). Allows a client to retrieve and update geospatial data from multiple Web Feature Services. [169]

All of the above mentioned requirements were addressed in the design and development of each of the CIPRsim HLA federates described in the following sections.

\section{$11.3 \quad$ Federates}

\subsubsection{Power Federate}

The electrical asset module, or Power Federate, is responsible for modeling power assets and their interconnections. The objective is to model accurately the effects of damages to the power components in the power grid by creating power models that accurately replicate the power grid in the areas selected for 
analysis. The power assets are modeled in RTDS (Real Time Digital Simulator) [170], which is a hardware/software system that models power systems.

The RTDS Simulator is a fully digital electromagnetic transient power system simulator that provides technology for a fast, reliable, accurate and cost-effective for the study of power systems with complex High Voltage Alternating Current (HVAC) and High Voltage Direct Current (HVDC) networks. It is a combination of advanced computer hardware and comprehensive software. The components of the system, including the graphical user interface (GUI), proprietary software and mathematical algorithms, can simulate any modern electric power grid configuration. RTDS provides the ability to run simulated system-failure scenarios such as a physical damage event caused by a natural disaster or terrorist attack and instantly determine why specific components within the electrical grid failed. The ability to simulate real-time power grid information is a key factor in providing emergency planners with a path forward for responding to grid failures.

To use RTDS, power models have to be created for each area of interest. The power models used in CIPRsim are created in RTDS by power engineers utilizing information gathered about the power grid in the geographic area being analyzed. Along with creating a power model in RTDS, information about each power asset (location, and type of power assets) must be gathered. This data is stored in ESRI shapefiles [171] to be used for visualization in the user interface with respect to location and state.

A mapping file is also created for each model that maps damages to assets to controls in RTDS and one that is used to create the logic for determining when an asset becomes inoperable based on information received from RTDS. The shape files, RTDS model file, and mapping files are stored on the computer where the power federate resides. The power federate loads a model when it receives a load model interaction from the run-time interface (RTI). It sends a load status interaction of success or failure for each load model request.

The Power Federate will follow these steps in loading a model:

- $\quad$ Publish a zip file of the shapefiles associated with the power assets

- $\quad$ Publish the assets on the RTI

- $\quad$ Create the mapping between assets and RTDS controls

- $\quad$ Send script commands to load the model in RTDS

- $\quad$ Send a script to start the model in RTDS

- Wait for the model to go into a steady state

- $\quad$ Connect to the OPC server and start collecting data from RTDS.

Power assets are damaged by receiving asset-affected messages from the RTI. The state of the asset is changed according to the message. Also, an attribute of the asset is set to include the reason the asset was damaged. For each asset that is damaged a corresponding set of commands is sent to RTDS to change the state of the model and reflect the damage in the power grid. When power assets are damaged it can cause cascading effects to occur in the power model. In order to know when an asset has failed, a file is created along with the power model in RTDS that denotes when an asset is no longer operable.

\subsubsection{Wireless Federate}

The wireless asset module, or Wireless Federate, is responsible for modeling wireless communications assets and their interconnections. The objective is to depict the effects of damages to the 
wireless communications components in the wireless network accurately. This is achieved by creating wireless communication models that replicate the wireless networks in the geographic areas selected for analysis. The wireless communications assets are modeled in QualNet [172], which is network modeling software that predicts real-time performance of wireless and wired networks through simulation and emulation.

To utilize QualNet, wireless network models must be created for each area of interest. The wireless models used in CIPRsim are created in QualNet by communications engineers utilizing information gathered about the wireless communications network in the geographic area being analyzed. Along with creating the wireless model in QualNet, information about each wireless asset, such as location of communication transceivers, their physical properties, connectivity, mobility, antenna configuration and orientation, and signal attenuation environment (e.g., due to terrain) must also be gathered. At startup, this data is assembled in four different ESRI shapefiles [171]; cellular towers, microwave links, mobile transceivers and cellular tower signal coverage area polygons (Figure 11-2: Wireless Coverage Polygons for INL Scenario).

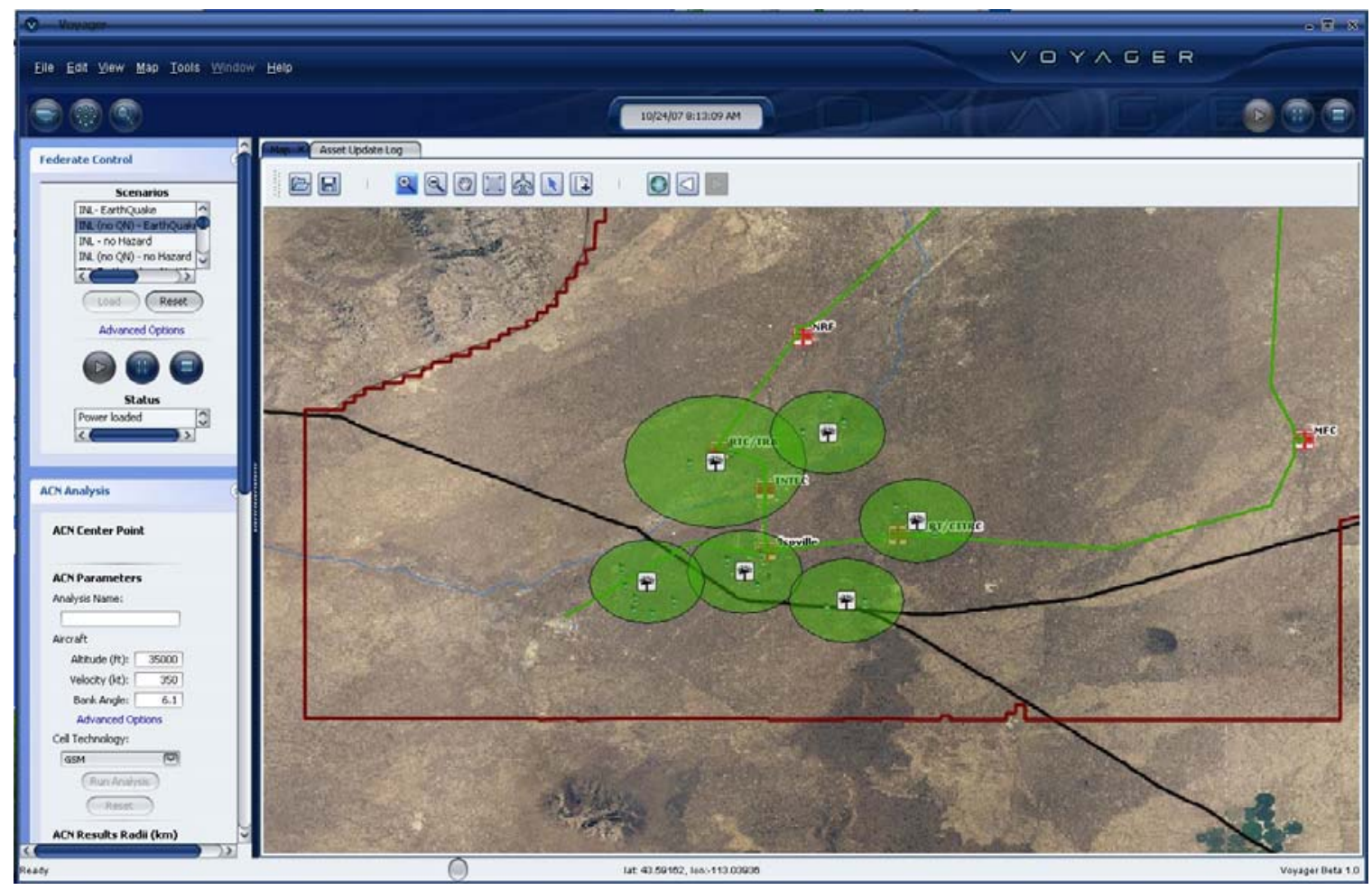

Figure 11-2: Wireless Coverage Polygons for INL Scenario

The shapefiles are sent to the user interface federate to visualize the geolocated radio transceivers and their coverage polygons as well as microwave links and mobile receivers. The coverage polygons are calculated in QualNet by running a simulation to steady state. Then, when a particular radio is depowered, for whatever reason, the GUI will simply clear that radio's polygon. QualNet will again run to steady state to compute further degradation caused by missed hops, dropped packets, etc, in the remaining network. Pre-hazard and post-hazard analyses provide the cell coverage maps for an area before and after an event occurs. The analyses will involve a static laydown of cell towers and their associated coverage areas. The only difference between pre-hazard and post-hazard is the laydown and the number of active cell towers. 


\subsubsection{Hazard and Damage Assessment Federate}

The hazard and damage assessment module, or Hazard and Damage Assessment Federate, is responsible for modeling the hazardous events in a scenario that act as initiators of infrastructure damage in the simulation. For this project the simulation is limited to hazards from hurricanes such as storm surge, flooding and high winds [163]and earthquakes such as ground acceleration.[164][153]

The hurricane model values are provided by PCTides. This is a global tide-surge forecast system composed of a 2-D barotropic ocean model driven by tidal forcing and/or wind and surface forcing. The system also includes a "hurricane model" developed by Holland. [162] Based on this model, a hurricane may be built that can provide surface pressures and winds to drive the 2-D barotropic ocean model and generate storm surge. The Holland model has been tested extensively and has been found to provide a good representation of wind fields near a tropical cyclone. [163] Figure 11-3: Example Wind Speeds and Storm Surge due to Hurricane shows an example of hurricane wind speeds and storm surge values generated from the PCTides models over the Tampa/St. Petersburg study area.

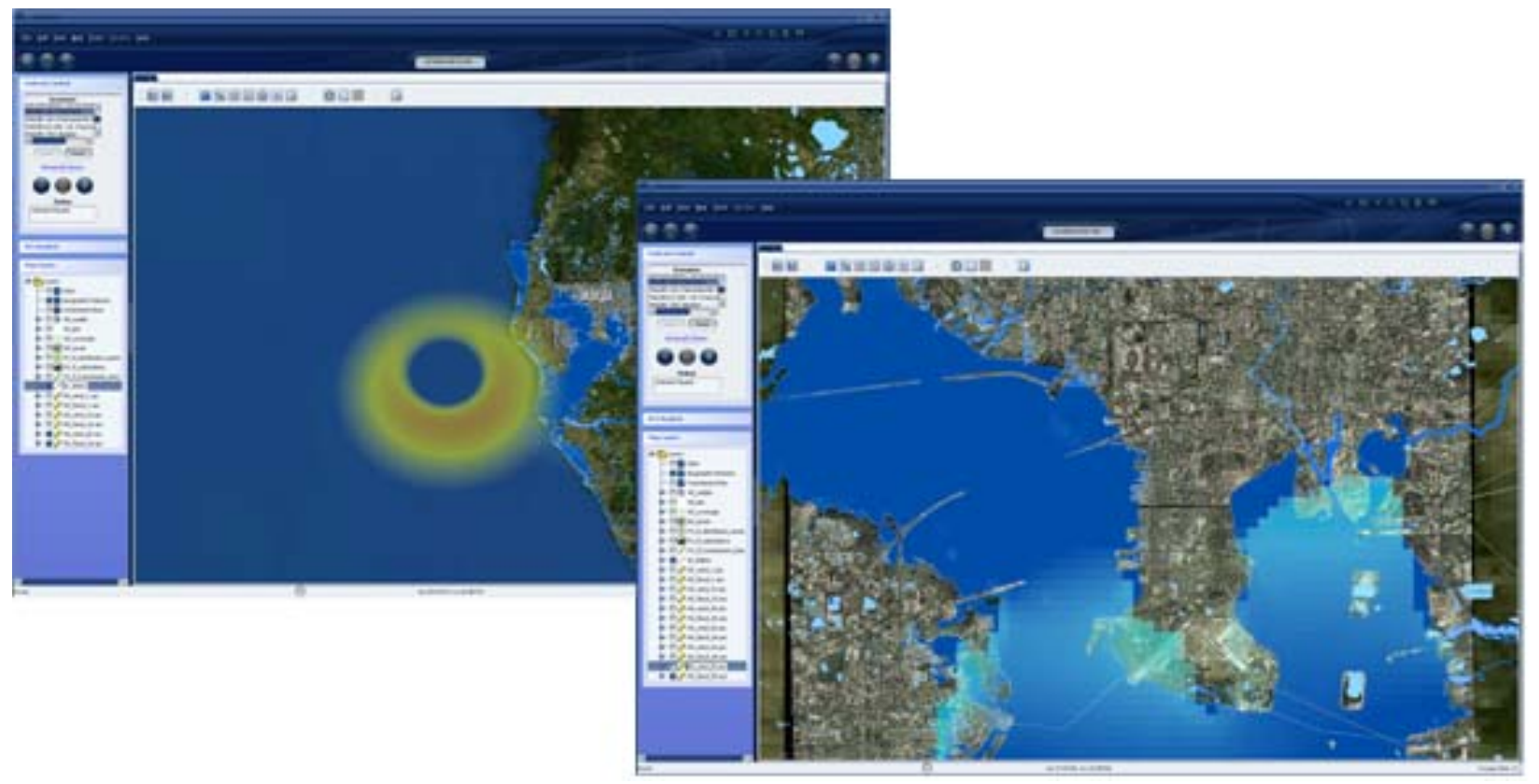

Figure 11-3: Example Wind Speeds and Storm Surge due to Hurricane

The earthquake model utilizes the equations developed by Boore, Joyner and Fumal[164][153], which give ground motion in terms of peak acceleration. While the current hazards are hurricanes and earthquakes, the framework is designed to support a full range of plausible hazards. This federate provides hazard temporal location and severity information to the other modules of the simulation. This in turn provides dynamic, time-varying effects of the hazard on the various infrastructure components at each time step in the model.

While both the hazard and damage assessment modules are contained in the same federate, they are distinct models with separate functionality. The hazard module produces hurricane and earthquake intensity levels based on contours generated by the disaster models. The damage assessment module is responsible for converting the provided intensity levels into component failures at the power and wireless federate level. Damage inflicted upon infrastructure assets by natural hazards is generally considered a probabilistic event. Neither RDTS nor QualNet are capable of accepting data or analyses in this fashion. Therefore, the hazard and damage assessment federate must provide component level failure information to the performance Federates using one of three standard states: operational, non-operational, or damaged 
(see Figure 11-4: Asset States). This methodology provides a way to supply deterministic failure information to the performance assessment modules at pre-defined probability of failure levels, thereby keeping the flavor of an overall probability-based model in the hazard and damage assessment arenas while complying with the requirements of the performance assessment modules in the CIPRsim architecture.

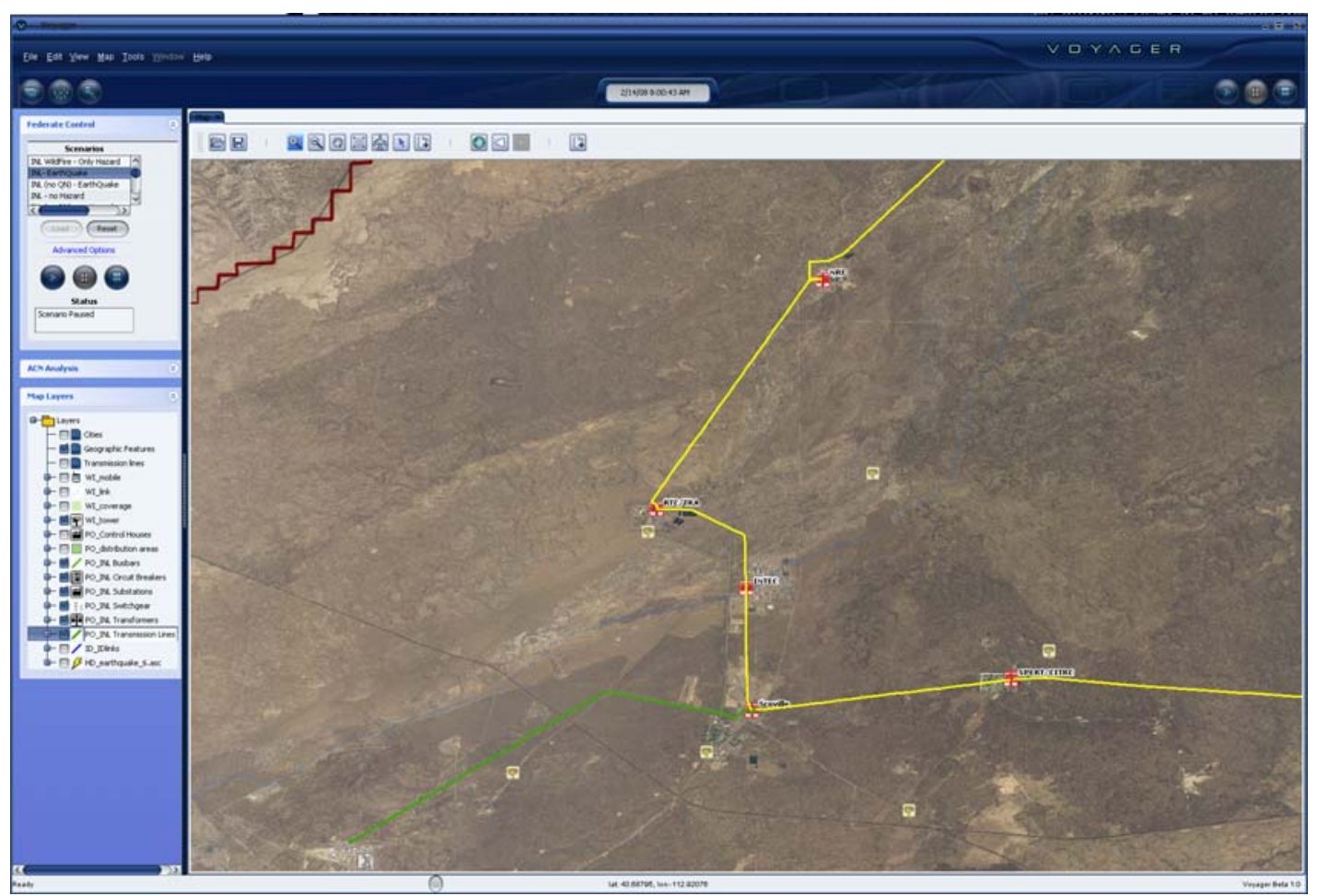

Figure 11-4: Asset States; Green-Operational, Yellow-Non-Operational, Red-Damaged

The damage assessment module will use the concept of fragility functions to describe the failure of infrastructure components. Fragility functions are cumulative probability density functions that provide the probability of failure at different hazard levels. An example fragility function is shown in Figure 11-5: Example Fragility Function. 


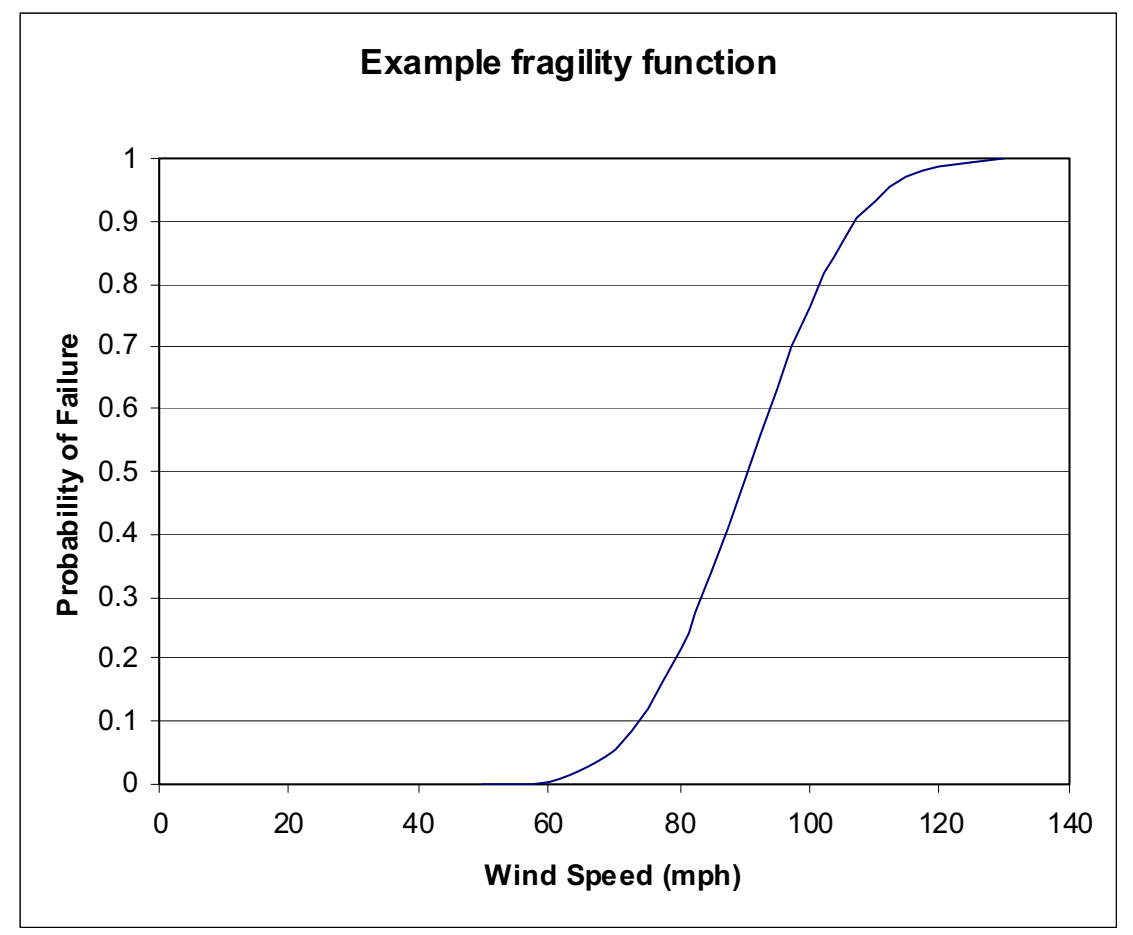

Figure 11-5: Example Fragility Function

The damage assessment module will use three types of fragility functions. Most damage assessments will be described by using a threshold-level Weibull distribution function [173], [174] however, the damage assessment module may use a straight-line distribution function, or a simple threshold-based failure model, which is a straight-line function with infinite slope. The form of the Weibull distribution function is shown below in Equation 11-1, Weibull Distribution.

$$
P_{f}=1-e^{\left(\frac{x-a}{b}\right)^{c}}, \text { where } \mathrm{x}>\mathrm{a}
$$

\section{Equation 11-1, Weibull Distribution}

Here, $\mathrm{x}$ represents the hazard level. The coefficients $\mathrm{a}, \mathrm{b}$, and $\mathrm{c}$ are constants that are used to adjust the threshold and shape of the distribution function. The constant $\boldsymbol{a}$ is the lower threshold of the function. The constant $\boldsymbol{b}$ is used to set the $63 \%$ failure level of the distribution with respect to the threshold value. The constant $c$ modifies how steep or fast the function approaches $100 \%$ failures. A larger exponent corresponds to a steeper function. An exponent of 3.5 approximates a normal distribution. Weibull functions are desirable because of they can be made to resemble a normal distribution function, are flexible, and are easy to configure.

\subsubsection{Interdependency Federate}

The interdependency federate provides the functionality to evaluate interdependencies and resulting cascading effects between the power and wireless federates. To accomplish this, the participating federates in the simulation publish their scenario-specific models to the HLA bus. Initial asset state changes are due to hazard and damage model affects on the assets. As mentioned in the previous sections, CIPRsim identifies three potential asset states. These include: 
- $\quad$ Fully Operational. Indicating that the asset is in working order.

- Non Operational. Indicating that an asset is not in working order, but with minor changes it can be brought back into use (i.e., power restored to a cell tower).

- $\quad$ Damaged. Indicating that an asset has been damaged and will need repair before it can be brought back into use (i.e., cell tower being knocked over).

These asset state changes are then published to the HLA bus where the Interdependency federate reads the new asset state changes due to hazard effects and evaluates them for interdependencies for local or physical interdependencies. Local interdependencies compare locations of participating model assets to evaluate possible areas of influence, depending on the type of assets that are being compared. The primary local interdependency analysis between the electrical and wireless models in the simulation involves electrical distribution service areas. These distribution service areas are based on the distribution-level electrical network that connects consumers (i.e., homes, businesses, factories, cell towers, etc.) to specific substations. Wireless assets are dependent upon these distribution areas due to a local interdependency. In other words, for a cell tower to have power:

- The substation providing power to the distribution area must be operational

- The distribution area in the vicinity of the substation must be operational

- $\quad$ The distribution area in the vicinity of the wireless asset must be operational.

Physical interdependencies indicate a direct, known physical connection between the two distinct participating models.

When an asset changes state, possible local or physical interdependencies are evaluated and an invitation for each affected asset will be posted to inform the affected asset's simulation, or federate, of the potential change of state. The posted invitation will include any state information that may help a participating model determine if the change is required, in addition to a suggestion for the new asset state. At this point, it is the responsibility of the model simulating the asset's behavior to determine whether or not the new state change is feasible, and then publish the appropriate asset status onto the HLA bus.

\subsubsection{User Interface}

The user interface federate provides the means by which the user interacts with the simulation and visualizes the results. It is composed of two main parts. The first component is the 2-D map-based visualization and analysis component, referred to as Voyager. Voyager is composed of two main parts Voyager Server and Voyager Client. End users will use the client to view and perform simulation tasks, while the server will publish simulation events to each client. The Voyager Client has a familiar Geographical Information System application look and feel similar to that of GoogleEarth ${ }^{\mathrm{TM}}$, and other common operating pictures such as the Department of Homeland Security's integrated Common Analytical Viewer (iCAV) application and the National Geospatial Intelligence Agency's Palanterra application (see Figure 11-6: Voyager Map-based Interface). 


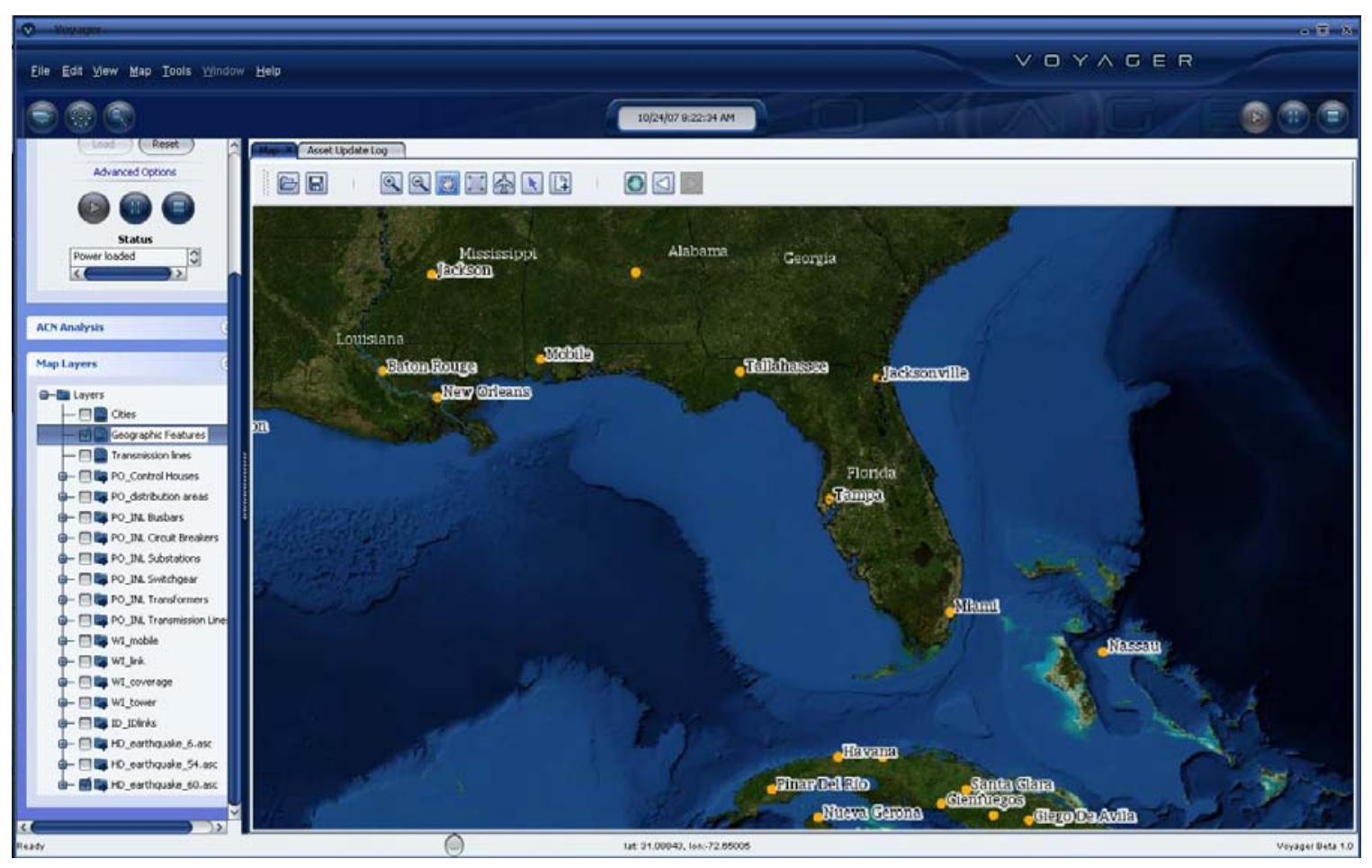

Figure 11-6: Voyager Map-based Interface

This Voyager client interface utilizes GeoTools [175], which is an open source Java GIS toolkit that provides implementations of many of the Open Geospatial Consortium (OGC) specifications identified as requirements during the design of the system. It also uses all of the common GIS navigation tools familiar to users including zooming, panning, data selection, layer control, etc. Voyager allows for importing geospatial data published by the other federates for visualization (see example in Figure 11-7: Federate Data Visualization). It shows storm surge information from the hazard and damage assessment federate overlaid with affected power and wireless assets published by the power federate and wireless federates. 


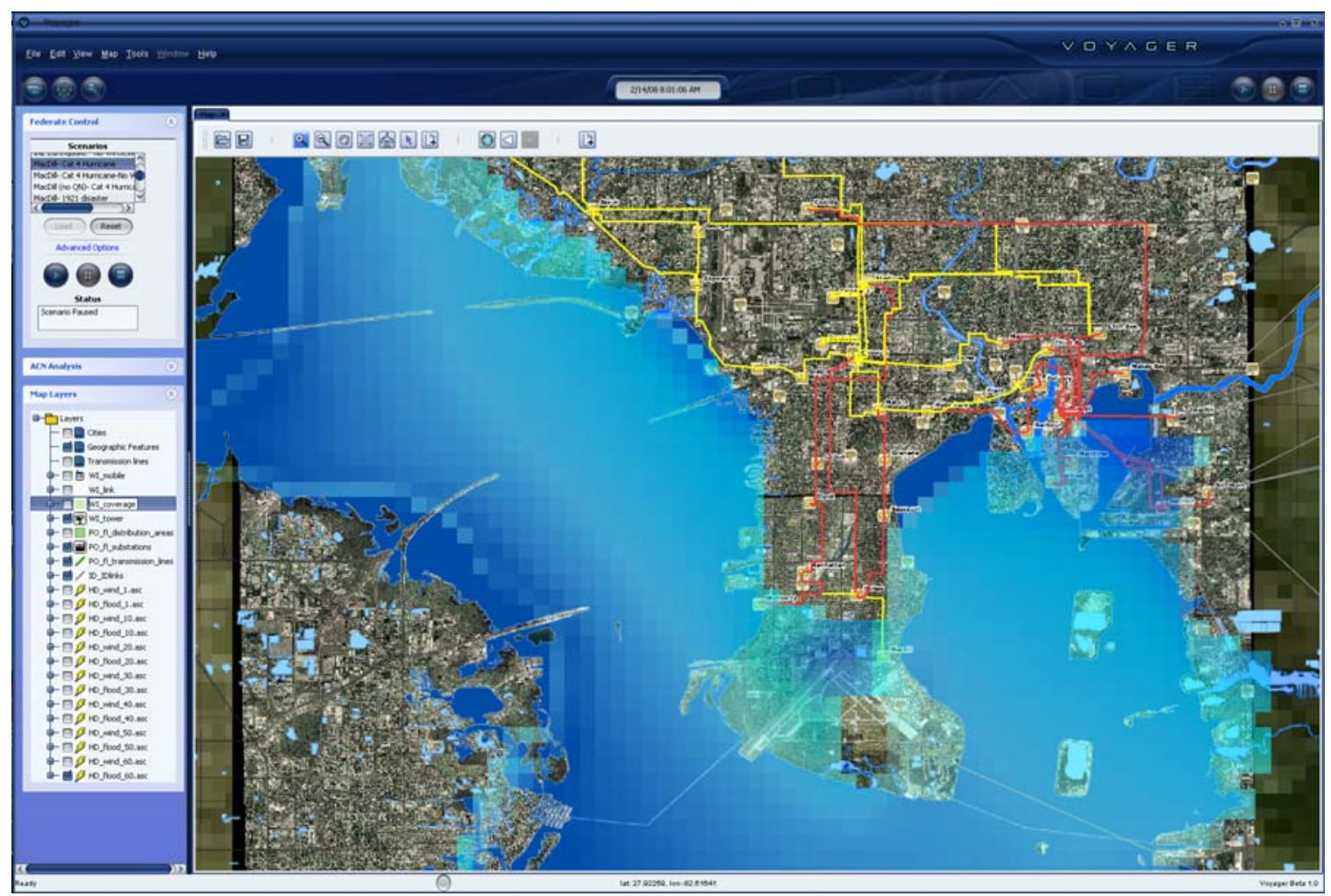

Figure 11-7: Federate Data Visualization

In addition to visualizing data published by the other federates in the simulation, the Voyager client provides the capability for analysis. It imports specific, user-defined data that may be used to enhance the results of the simulation through increased situational awareness based upon proximity to occurring events. The Voyager server utilizes GeoServer [176], an open source service architecture that allows for viewing and editing geographic data via the Web. This includes publishing data as maps or images using the OGC WMS specification [167], and actual data such as transmission lines, substations or cellular towers using the OGC Web Feature Service specification. [169] This functionality within the user interface and visualization module supports the collection of disparate data from military and civilian sources as well as the distribution of the CIPRsim results back to these communities. This includes data feeds from external servers to allow for access to accurate and current data not only from the DCIP community but also from compatible federal interagency nodes. Another reason for this functionality is to ensure interoperability with existing end-user community situational awareness, analysis and visualization systems through the ability to both read and export data in industry standard formats. Due to these interoperability requirements, special emphasis is placed on leveraging the availability of COTS/GOTS products and interfacing with tools and capabilities already employed by identified end-users.

In addition to its data and information visualization function, the 2-D Voyager client acts as the overall simulation control by allowing the operator to choose a scenario, and start, stop or pause the simulation. The operator may also identify assets and view their current state (see Figure 11-8), and view a $\log$ of simulation events throughout the course of the simulation that provides a "play-by-play" record of what happened and when it happened to each of the assets of interest (see Figure 11-9). 


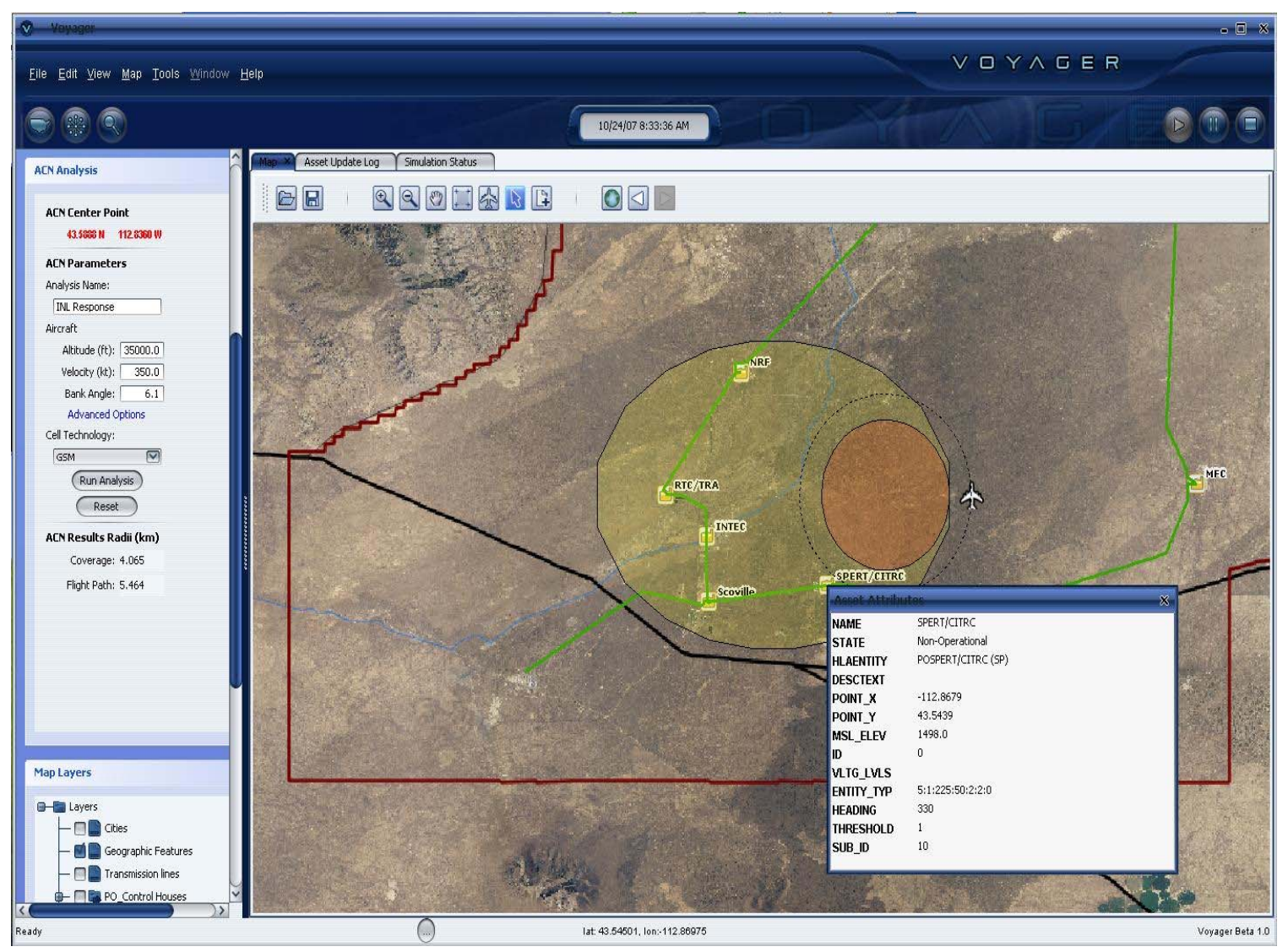

Figure 11-8: Asset Identification and State Information

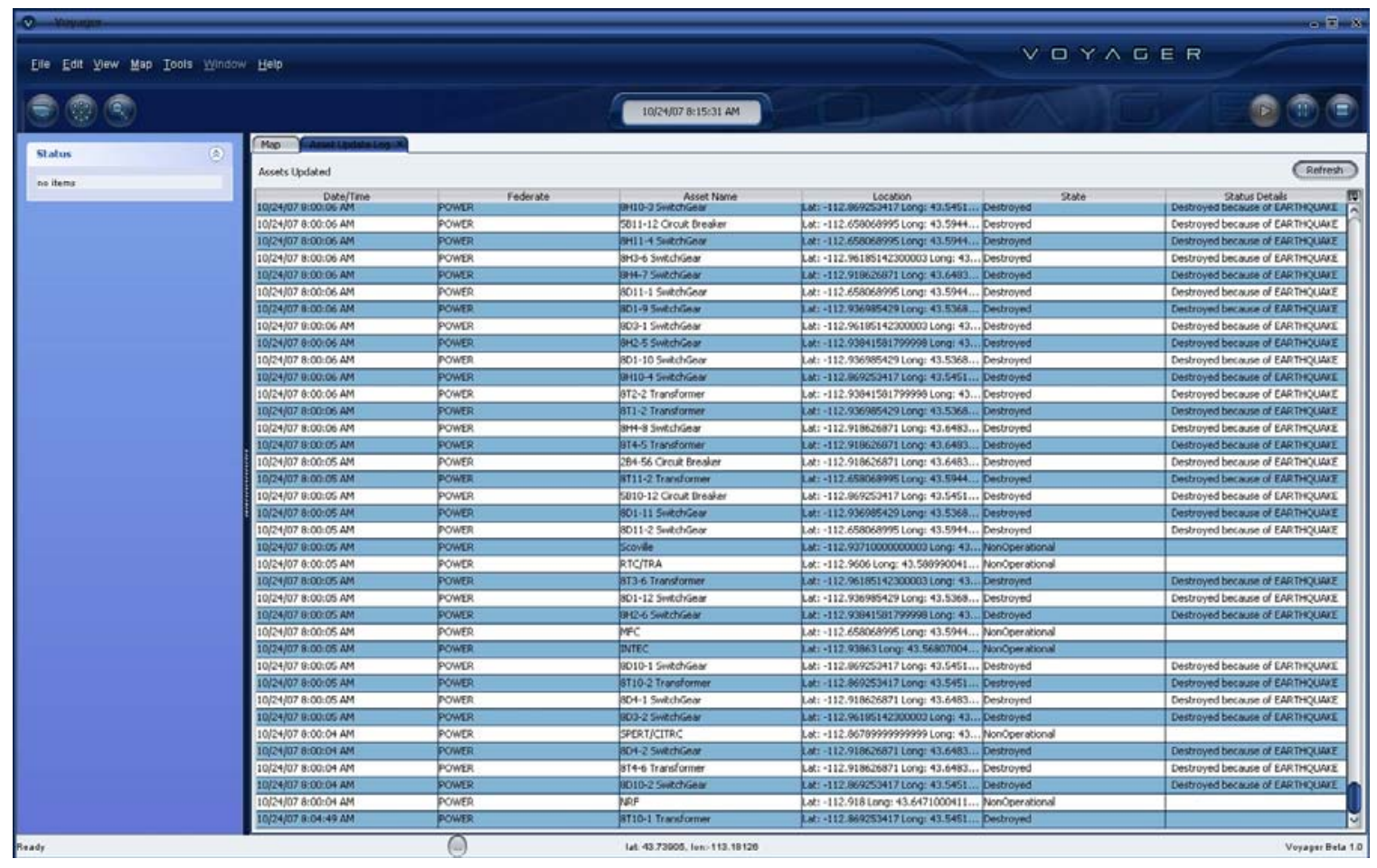

Figure 11-9. Asset Event Log 
The final function of significance related to the Voyager client concerns the ACN analysis. This component provides ACN coverage calculations used for communications restoration pblanning and recovery operations as well as visualization of the analysis results (see Figure 11-10).

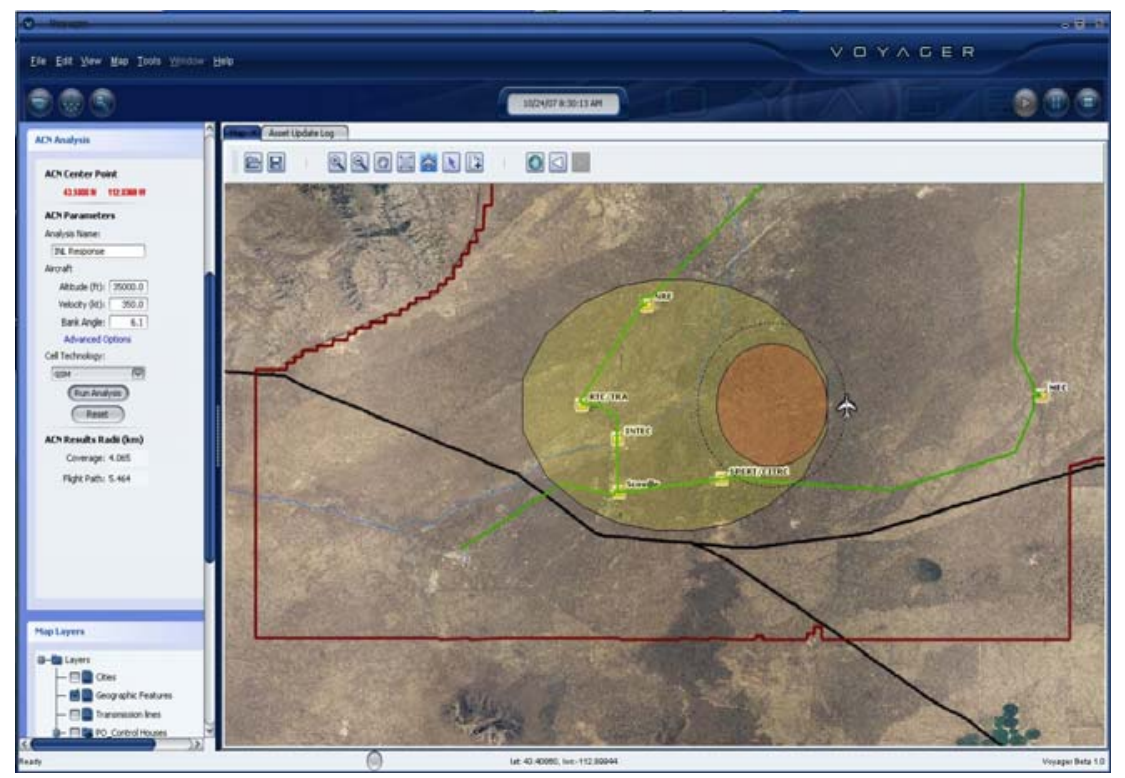

Figure 11-10: ACN Continuous and Instantaneous Coverage Areas

Inputs to this analysis include:

1. Starting position of the ACN platform, which is determined by selecting a point on the map

2. Altitude at which the ACN platform will fly

3. Velocity at which the ACN platform will fly

4. Bank angle of the ACN platform

5. Cellular technology (CDMA or GSM) incorporated on the CAN.

The outputs from the analysis module consists of:

1. ACN restoration areas, including continuous coverage area and instantaneous coverage area given the ACN input parameters, are drawn and rendered on the map (see Figure 11-10).

The second component of the user interface consists of a 3-D visualization capability, which allows an analyst the ability to view, in simulation-time, the aspects of the occurring event in a fluid and animated visual, similar to popular gaming environments involving 3D graphics models and their autonomy. This allows the analyst the opportunity to "see" the simulation in multiple layers and multiple camera views simultaneously, so that (1) situational awareness increases, and (2) analytical focus may be achieved. This 3D environment connects as a federate to the RTI so that, as events are received, the 3D visualization will show the events graphically as they occur. The 3-D visualization component utilizes MÄK Technology's Stealth 3-D Visualization engine. [177] This component contains a 3-D visualization engine, which includes 3-D graphics models with the ability for animations that are affected by state changes received from the simulation federates. 
The 3D graphics included within the 3D interface consist of background graphics such as terrain and building structures, and 3-D depictions of the cellular infrastructure (see Figure 11-11), 3-D depictions of the electrical infrastructure (see Figure 11-12) and 3-D depictions of the ACN platform in flight (see Figure 11-13).

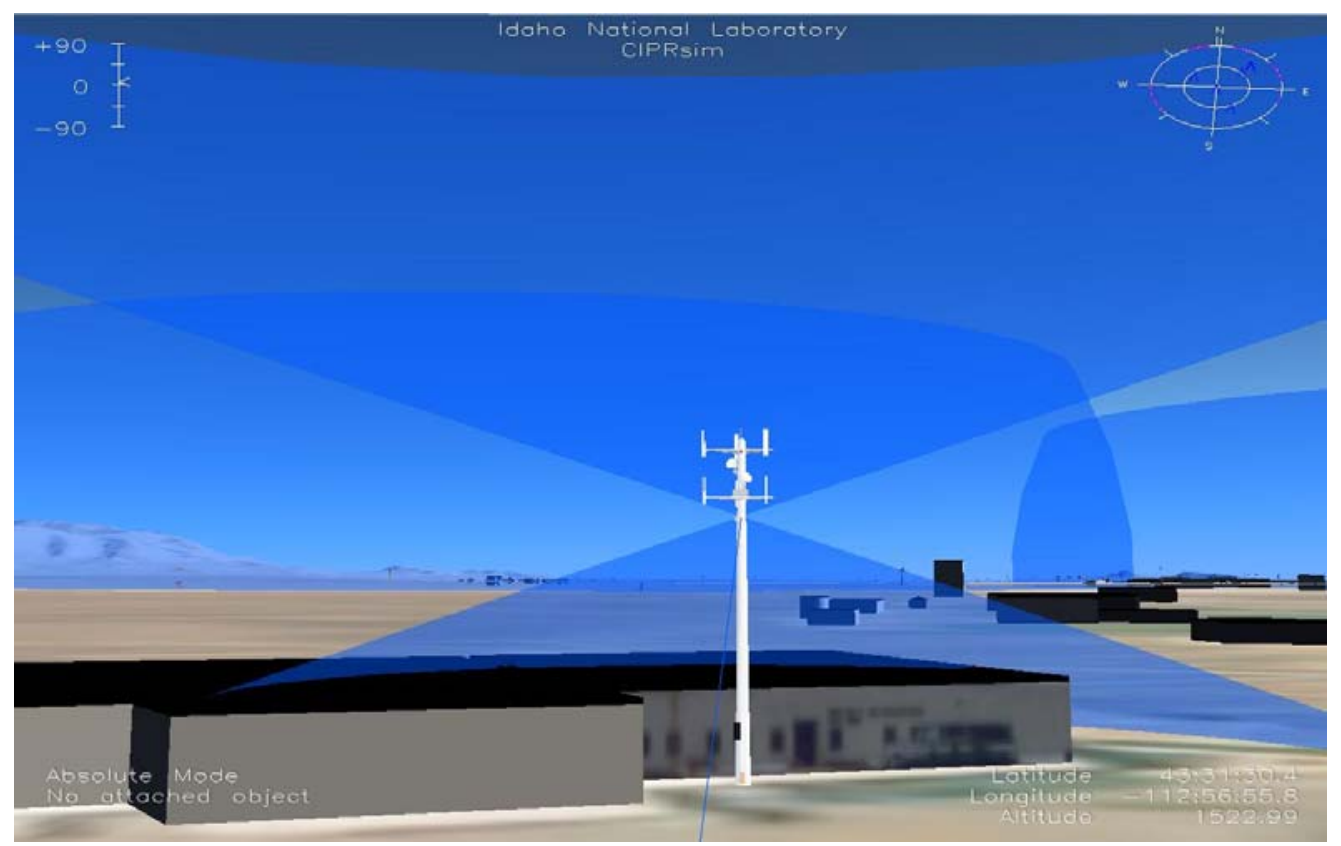

Figure 11-11: 3-D depiction of Cellular Infrastructure

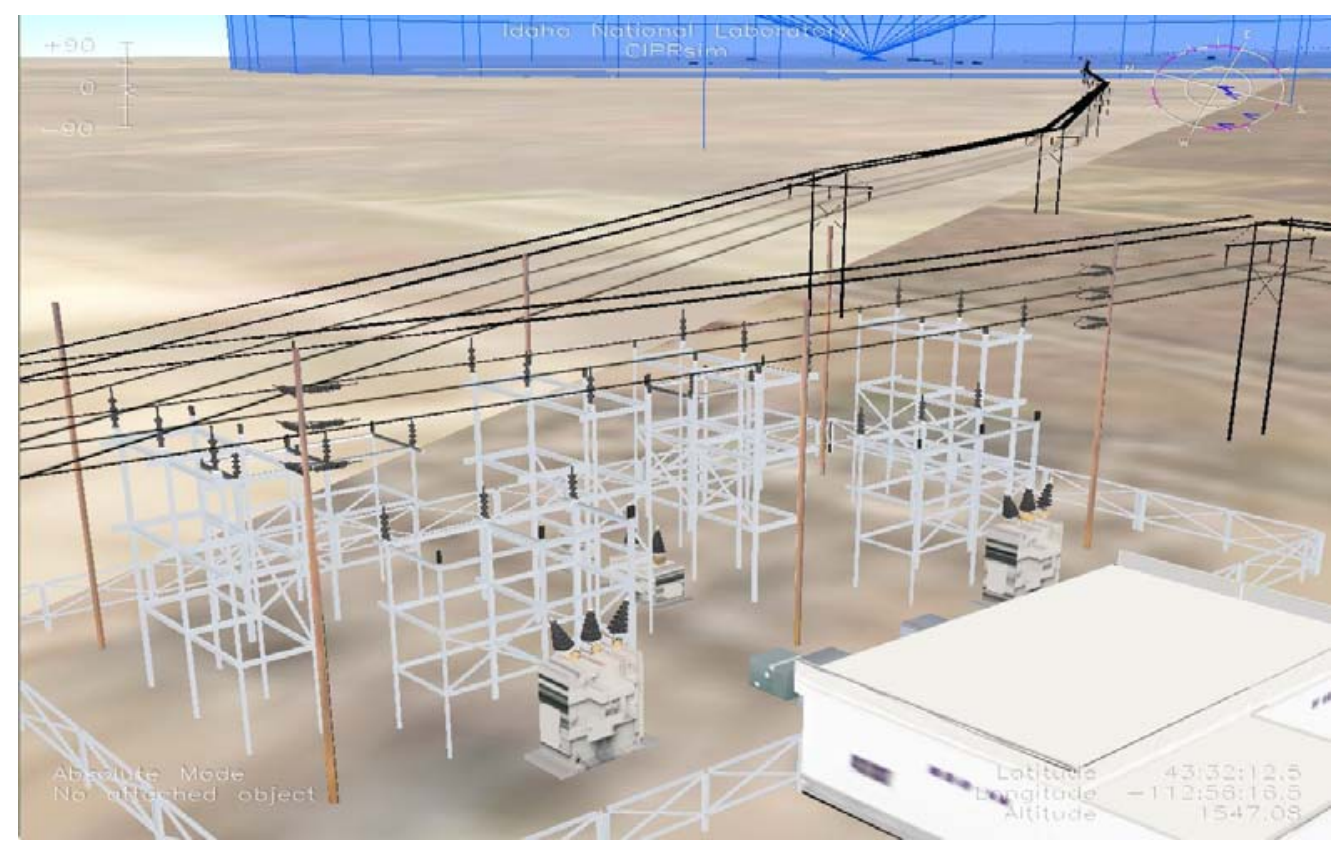

Figure 11-12: 3-D Depiction of Electrical Infrastructure 


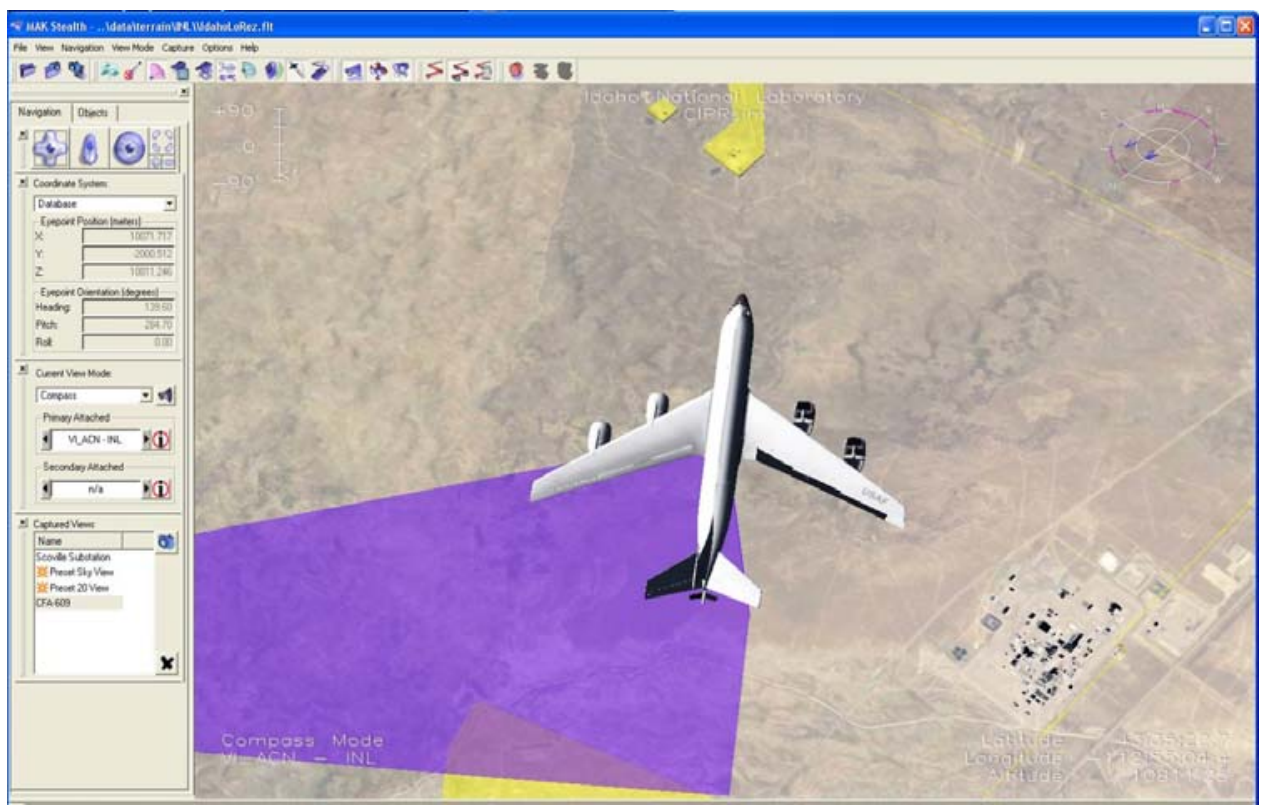

Figure 11-13: 3-D Depiction of ACN in Flight 


\section{REFERENCES}

[153] National Security Telecommunications Advisory Committee, "NSTAC Repot to the President on Telecommunications and Electric Power Interdependencies - The Implications of Long Term Outages," December 19, 2006.

[154] Pederson, P., D. Dudenhoeffer, S. Hartley, and M. Permann, 2006, "Critical Infrastructure Interdependency Modeling: A Survey of U.S. and International Research," Prepared for the Technical Support Working Group.

[155] Rinaldi, S. M., 2004, "Modeling and Simulating Critical Infrastructures and Their Interdependencies," Proceedings of the $37^{\text {th }}$ Hawaii International Conference on System Sciences.

[156] National Security Telecommunications Advisory Committee, Telecommunications and Electric Power Interdependency Task Force, "People and Processes: Current State of Telecommunications and Electric Power Interdependencies," January 31, 2006.

[157] Kuhl, F., R. Weatherly, and J. Dahmann, 1999, Creating Computer Simulation System - An Introduction to the High Level Architecture, Upper Saddle River, NJ. Prentice Hall, ISBN 0130225118.

[158] Tolk, A., 2002, “Avoiding Another Green Elephant - A Proposal for the Next Generation HLA Based on the Model Driven Architecture," 2002 Fall Simulation Interoperability Workshop (SIW), Orlando, FL, September 2002.

[159] Jain, S. and C. McLean, 2003, "A Framework for Modeling and Simulation for Emergency Response," Proceedings of the 2003 Winter Simulation Conference.

[160] Coolahan, J. E., 2006, "Planning for an Integrated M\&S Framework for Catastrophic Event Response," http://www.pacercenter.org/pdf/coolahanPublication.pdf

[161] Coolahan, J. E., M. T. Kane, J. F. Schloman, R. P. Koomullil, A. M. Shih, I. Yasushi, K. I. Evangelos, K.K. Walsh, and M.M. Abdullah, 2007, "Design of an Urban Chemical Disaster Simulation Federation for Preparedness and Response," 2007 Fall Simulation Interoperability Workshop (SIW), Orlando, Florida, September 2007.

[162] Holland, G. J., 1980, "An Analytical Model of the Wind and Pressure Profiles in Hurricanes," Mon. Wea. Rev., 108, 1212-1218.

[163] Preller, R. H., P. G. Posey, and G. M. Dawson, "Hurricane Isabel: A Numerical Model Study of Storm Surge Along the East Coast of the United States," National Research Laboratory, Stennis Space Center, Mississippi, NRL/PP/7320-04-5010.

[164] Boore, D. M., W. B. Joyner, and T. E. Fumal, 1997, "Equations for Estimating Horizontal Response Spectra and Peak Acceleration from Western North America Earthquakes: A Summary of Recent Work," Seismological Research Letters, Volume 68, Number 1, January/February 1997. 
[165] Office of the Assistant Secretary of Defense for Homeland Defense - OASD(HD) Critical Infrastructure Protection (CIP), 2006, "Defense Critical Infrastructure Program (DCIP) Geospatial Data Strategy," http://www.defenselink.mil/policy/sections/policy offices/hd/assets/downloads/dcip/DCIP Geospa $\underline{\text { tial_Data_Strategy.pdf }}$

[166] National Geospatial Intelligence Agency, 2006, "Geospatial Intelligence Standards - Enabling a Common Vision,” http://www.nga.mil/NGASiteContent/StaticFiles/OCR/ncgis-eb.pdf

[167] Open Geospatial Consortium, 2006, "OpenGIS Web Map Server Implementation Specification," Reference Number: OGC 06-042.

[168] Open Geospatial Consortium, 2006, "Web Coverage Service Implementation Specification," Reference Number: OGC 06-083r8.

[169] Open Geospatial Consortium, 2005, "Web Feature Service Implementation Specification," Reference Number: OGC 04-094.

[170] RTDS Technologies, http://www.rtds.com/

[171] ESRI, "ESRI Shapefile Technical Description," http://www.esri.com/library/whitepapers/pdfs/shapefile.pdf

[172] Scalable Network Technologies, http://www.scalable-networks.com/

[173] Weibull, W., 1951, "A Statistical Distribution Function of Wide Applicability," J. Appl. Mech.Trans. ASME 18(3), 293-297.

[174] National Institute of Standards and Technology, Engineering Statistics Handbook, http://www.itl.nist.gov/div898/handbook/apr/section1/apr162.htm

[175] GeoTools, http://geotools.codehaus.org/

[176] GeoServer, http://geoserver.org/

[177] MÄK Technologies, http://www.mak.com/

[178] Jain, S. and C. McLean, 2005, "Integrated Simulation and Gaming Architecture for Incident Management Training," Proceedings of the 2005 Winter Simulation Conference.

[179] Wytzisk, A., I. Simonis, and U. Raape, 2003, "Integration of HLA Simulation Models into a Standardized Web Service World." http://ifgi.unimuenster.de/ $\sim$ simonis/download/eurosiw2003.pdf 


\section{ACRONYMS}

$\begin{array}{ll}\text { CI } & \text { Critical Infrastructure } \\ \text { CIPRsim } & \text { Critical Infrastructure Protection/Resilience Simulator } \\ \text { COP } & \text { Common Operating Picture } \\ \text { DCIP } & \text { Defense Critical Infrastructure Program } \\ \text { FGDC } & \text { Federal Geographic Data Committee } \\ \text { GEOINT } & \text { Geospatial Intelligence } \\ \text { GIS } & \text { Geographical Information System } \\ \text { HLA } & \text { High Level Architecture } \\ \text { iCAV } & \text { Integrated Common Analytical Viewer } \\ \text { IEEE } & \text { Institute of Electrical and Electronics Engineers } \\ \text { KR } & \text { Key Resource } \\ \text { M\&S } & \text { Modeling and Simulation } \\ \text { NSTAC } & \text { National Security Telecommunications Advisory Committee } \\ \text { OGC } & \text { Open Geospatial Consortium } \\ \text { RTDS } & \text { Real Time Digital Simulator } \\ \text { NGA } & \text { National Geospatial Intelligence Agency } \\ \text { RTI } & \text { Run Time Interface } \\ \text { SMADS } & \text { STRATCOM Mission Assurance Data System } \\ \text { U.S. STRATCOM } & \text { United States Strategic Command } \\ \text { WCS } & \text { Web Coverage Service } \\ \text { WFS } & \text { Web Feature Service } \\ \text { WFS-T } & \text { Web Feature Service - Transactional } \\ \text { WMS } & \text { Web Map Service } \\ & \end{array}$

